\title{
Transient prehypertensive treatment : an additive option against hypertension
}

Citation for published version (APA):

Baumann, M. (2007). Transient prehypertensive treatment : an additive option against hypertension. [Doctoral Thesis, Maastricht University]. Universitaire Press Maastricht.

https://doi.org/10.26481/dis.20071031mb

Document status and date:

Published: 01/01/2007

DOI:

$10.26481 / \mathrm{dis} .20071031 \mathrm{mb}$

Document Version:

Publisher's PDF, also known as Version of record

\section{Please check the document version of this publication:}

- A submitted manuscript is the version of the article upon submission and before peer-review. There can be important differences between the submitted version and the official published version of record.

People interested in the research are advised to contact the author for the final version of the publication, or visit the DOI to the publisher's website.

- The final author version and the galley proof are versions of the publication after peer review.

- The final published version features the final layout of the paper including the volume, issue and page numbers.

Link to publication

\footnotetext{
General rights rights.

- You may freely distribute the URL identifying the publication in the public portal. please follow below link for the End User Agreement:

www.umlib.nl/taverne-license

Take down policy

If you believe that this document breaches copyright please contact us at:

repository@maastrichtuniversity.nl

providing details and we will investigate your claim.
}

Copyright and moral rights for the publications made accessible in the public portal are retained by the authors and/or other copyright owners and it is a condition of accessing publications that users recognise and abide by the legal requirements associated with these

- Users may download and print one copy of any publication from the public portal for the purpose of private study or research.

- You may not further distribute the material or use it for any profit-making activity or commercial gain

If the publication is distributed under the terms of Article $25 \mathrm{fa}$ of the Dutch Copyright Act, indicated by the "Taverne" license above, 
Transient prehypertensive treatment:

An additive option against hypertension? 
(c) Marcus Baumann, Maastricht 2007

Cover Design: Roger Bartholome, Zhou Shi-Hui

Layout: Marcus Baumann

Production: Datawyse I Universitaire Press Maastricht

The studies presented in this thesis were funded by an EU Marie Curie grant, the Dutch Kidney Foundation and performed within the Cardiovascular Research Institute Maastricht (CARIM).

Financial support for printing of this thesis has kindly been provided by the Dutch Kidney Foundation. 


\title{
Transient prehypertensive treatment:
}

\section{An additive option against hypertension?}

\author{
PROEFSCHRIFT
}

Ter verkrijging van de graad van doctor

aan de Universiteit Maastricht

op gezag van de Rector magnificus, Prof. mr. G.P.M.F. Mols,

volgens het besluit van het college van Decanen,

in het openbaar te verdedigen

op woensdag 31 oktober 2007 om 16.00 uur

door

Marcus Baumann

Geboren op 29 oktober 1973 te Wuppertal 


\section{Promotores:}

Prof. Dr. H.A.J. Struijker Boudier

Prof. Dr. J.F.M. Smits

\section{Committee:}

Prof. Dr. P. de Leeuw (chairman)

Prof. Dr. J.W. Cohen Tervaert

Prof. Dr. U. Heemann

Prof. Dr. J. Staessen

Prof. Dr. Y. Pinto 


\section{CONTENTS}

INTRODUCTION

Definition of Hypertension 9

Current antihypertensive treatment strategy

When to initiate treatment 11

Goals of treatment 13

How to initiate treatment? 14

Antihypertensive treatment

Current effectiveness of treatment strategies 16

The costs of therapeutic strategies 17

General thoughts regarding therapeutic concepts 18

Role of the kidney in hypertension in humans 19

The concept of a primary renovascular disease causing essential hypertension 19

Primary renal abnormalities in hypertension 20

Role of renal vasculature 22

Renal ischemia revisited in animal models 23

Salt sensitivity in animal models 23

SHR as an animal model to investigate the development of hypertension 24

Transient prehypertensive treatment in SHR 25

Choice of drugs in early development of hypertension 28

Aims of the thesis 28

CHAPTER 2: Transient AT1 receptor-inhibition in prehypertensive SHR results in maintained cardiac protection until advanced age

CHAPTER 3: Prehypertensive RAAS blockade in SHR ameliorates long-term vascular function 47

CHAPTER 4: Sustained renoprotection in SHR by transient prehypertensive losartan treatment: an effect of decelerated aging? 61

CHAPTER 5: Functional and structural postglomerular alterations in the kidney of prehypertensive spontaneously hypertensive rats 
CHAPTER 6: The renal medullary microcirculation as drug target for prehypertensive treatment in young $S H R$

CHAPTER 7: Potential individual prediction of the progression form prehypertension to new-onset hypertension

GENERAL DISCUSSION AND CONCLUSIONS 107

Potential of prehypertensive treatment in the SHR

Blood pressure lowering

107

Target organ protection

Blood pressure independent effects

A shift in RAAS sensitivity as a concept for prolonged target organ protection

Structural alterations in the renal medulla 110

Renal medullary pericytes regulate blood pressure 111

Pharmacological Perspective 112

When to perform transient prehypertensive treatment in humans - a translational approach

Marker of the development of hypertension

Performing a case control study 113

The future of transient prehypertensive treatment 114

SUMMARY 


\section{INTRODUCTION}

\section{Definition of Hypertension}

'Hypertension should be defined in terms of a blood pressure level above which investigation and treatment do more good than harm'. This comment by ROSE points to the fact that a continuous relationship exists between the level of blood pressure and cardiovascular risk that makes any numerical definition and classification of hypertension arbitrary.

The World Health Organization (WHO) in collaboration with the International Society of Hypertension (ISH) constructed in 1999 a scheme covering several grades of blood pressure including optimal, normal, high-normal blood pressure and mild, moderate and severe hypertension (1) (Table 1.1).

Table 1.1 (adapted from ESH guidelines)

\begin{tabular}{|c|c|c|}
\hline Catagury & Systolic & Dizlytolic \\
\hline Optimal & $\therefore 120$ & $\therefore 80$ \\
\hline Normal & $120-128$ & $80-84$ \\
\hline High normal & $130-130$ & $85-89$ \\
\hline Grade 1 hyperteneson (mild & $140-159$ & $90-99$ \\
\hline Grade 2 hypertersian (moderate) & $160-179$ & $100-100$ \\
\hline Grade 3 hypertenson (severes & 180 & $=110$ \\
\hline Boolatad systalic hypertenstan & $=140$ & $\because 90$ \\
\hline
\end{tabular}

Related to the WHO/ISH scheme there are two additional major guidelines either based on the European Society of Hypertension (ESH guidelines 2003) or the American Society of Hypertension (Joint National Committee on the Prevention, Detection, Evaluation and Treatment of High Blood Pressure, JNC-VII). One difference exists in the nomenclature of the American and European guidelines. The Americans additionally use the term prehypertensive for individuals with blood pressure values between $120-139 \mathrm{~mm} \mathrm{Hg}$ systolic or $80-89 \mathrm{~mm} \mathrm{Hg}$ diastolic. 
These two international guidelines extend the $\mathrm{WHO} / \mathrm{SH}$ guidelines by including additional risk stratifications and offering therapeutic intervention thresholds for the treatment of cardiovascular risk factors such as blood pressure.. Because risk factors cluster in individuals $(2,3)$ and there is a graded association between each risk factor and overall cardiovascular risk (4), the contemporary approach to treatment is to determine the threshold, at least for cholesterol and blood pressure reduction, based on the calculation of estimated coronary or cardiovascular (coronary plus stroke) (5) risk over a defined, relatively short-term (e.g. 5- or 10-year) period.

\section{Development of Hypertension}

Hypertension is an important worldwide public-health challenge because of its high frequency and concomitant risks of cardiovascular and kidney disease. It has been identified as the leading risk factor for mortality, and is ranked third as a cause of disability-adjusted life-years (6).

Population-based analyses indicated that more than a quarter of the world's adult population had hypertension in 2000 and that this proportion will increase to $29 \%$, that means 1.56 billion, by 2025 . Men and women have similar prevalence of hypertension, and such prevalences increase with age consistently in all world regions.

The high prevalence of hypertension worldwide has contributed to the present pandemic of cardiovascular disease. Cardiovascular diseases are responsible for $30 \%$ of all death worldwide. Furthermore, hypertension affects more people in economically developing than developed countries (7). The proportion of deaths from cardiovascular disease before age 70 years was $46.7 \%$ in economically developing countries compared with $26.5 \%$ in developed countries (8).

The relation between cardiovascular outcome and blood pressure based on Framingham data were thought log-linear without a critical level above which the risk suddenly rises. As pointed out by the WHO/SH scheme a continuum of blood pressure values exists which complicates the management of hypertension for the clinician, as he needs operational thresholds.

The rate of progression of prehypertension to hypertension can be relatively rapid, particularly in those whose blood pressures lie in the upper portion of the prehypertension range and in elderly individuals (9). In Framingham Study participants with blood pressure levels in the 120 to $129 / 80$ to $84 \mathrm{~mm} \mathrm{Hg}$ range, the blood pressure 
progressed over 4 years to hypertensive levels in $17.6 \%$ of individuals between 30 and 64 years of age and in $25.5 \%$ of those $>65$ years of age (10). In the group with blood pressure levels in the 130 to $139 / 85$ to $89 \mathrm{~mm} \mathrm{Hg}$ range, the incidence of hypertension was $37.3 \%$ and $49.5 \%$ for the 30 - to 64 -year and $>65$-year groups, respectively. Furthermore it has been demonstrated that prehypertensive subjects demonstrate an increased cardiovascular risk as compared to subjects with optimal blood pressure.

Table 1.2 (adapted from ESH Guidelines)

\begin{tabular}{|c|c|c|c|c|c|}
\hline \multirow[b]{2}{*}{ 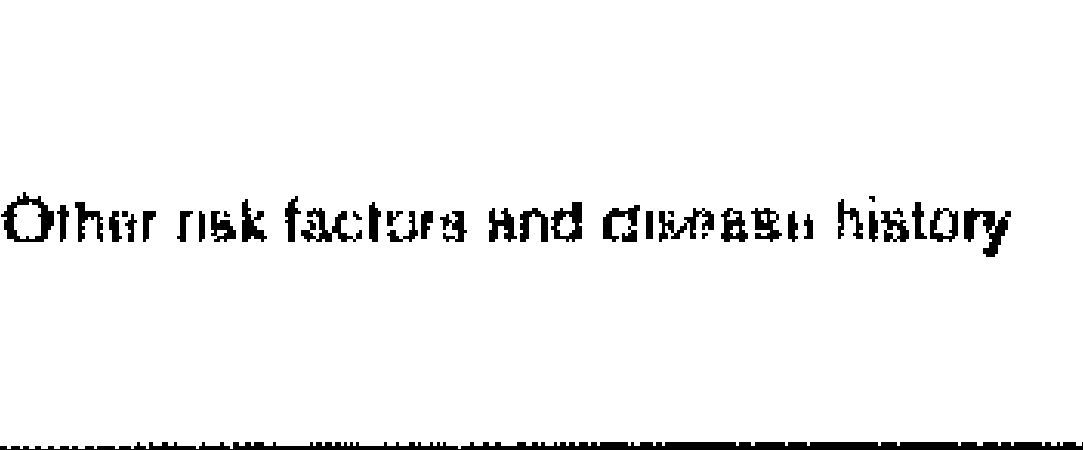 } & \multicolumn{5}{|c|}{ Biosd prestaure (mmHg) } \\
\hline & $\begin{array}{l}\text { Nermad } \\
\text { SBP } 120-129 \\
\text { or DeP BO..84 }\end{array}$ & $\begin{array}{l}\text { High nsmmal } \\
\text { SBP } 130-139 \\
\text { tor DEP } 85-89\end{array}$ & $\begin{array}{l}\text { Grade } \\
\text { SBP } 140-158 \\
\text { OXPP } 90-00\end{array}$ & $\begin{array}{l}\text { Grada } 2 \\
\operatorname{SeP} 180 \cdots 179 \\
\text { Gr DEP } 100-100\end{array}$ & $\begin{array}{l}\text { Gragde } 3 \\
\text { SBP in } 180 \\
\text { or DBP } .110\end{array}$ \\
\hline Wo other hak factors & 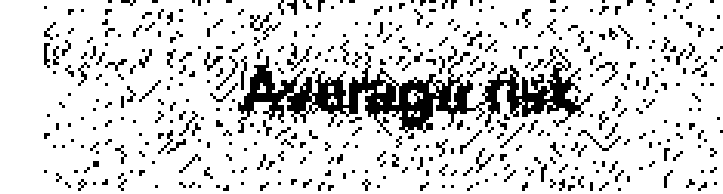 & - & Low ochded riak. & Morde'at? adsara II9k & High addiad rigk \\
\hline $1 \ldots 2$ flik factors & Low sodtad riak & Low adthed tilak & Mosferatis adowe risk & Mordataty adteked nak & 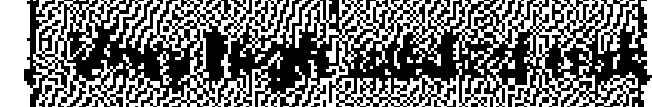 \\
\hline 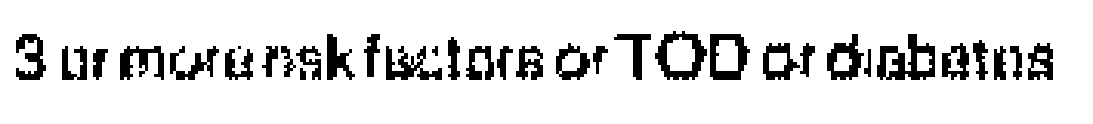 & Moderata added rigx & High added rivk & Hight vadded hisk & High acded riak & 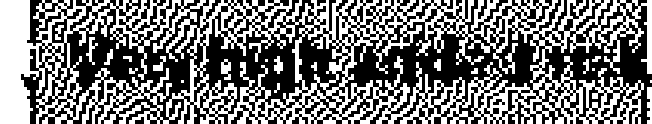 \\
\hline $\mathrm{ACC}$ & High addad risk & 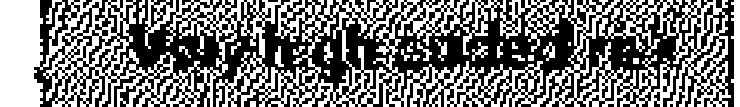 & W & 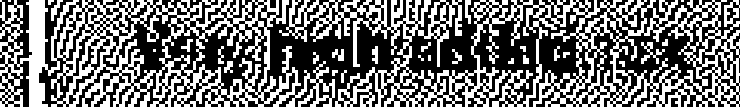 & 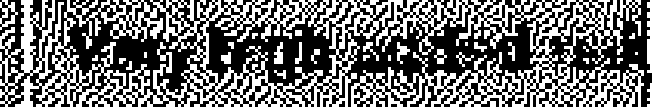 \\
\hline
\end{tabular}

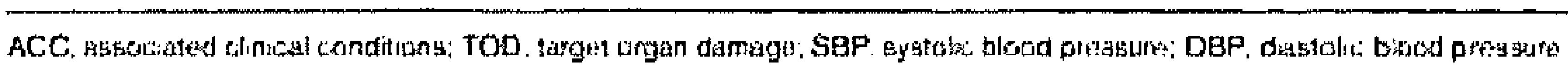

The size of this problem is demonstrated by the fact that approximately $60 \%$ of American adults have high normal blood pressure or hypertension, and some population groups, such as African Americans, older people, low-socioeconomic-status groups, and overweight groups, are disproportionately affected. Therefore $60 \%$ of the American population demonstrates an increased cardiovascular risk (11).

\section{Current antihypertensive treatment strategy When to initiate treatment}

Guidelines for initiating antihypertensive treatment are based on two criteria: (1) the total level of cardiovascular risk, as indicated in Table 1.2; and (2) the level of systolic and diastolic blood pressure. The total level of cardiovascular risk is the main indication for intervention, but lower or higher blood pressure values are also more or less stringent indicators for blood-pressure-lowering intervention. 
With respect to previous guidelines of the European Societies $(12,13)$ or the WHO/ISH (1), the recommendations summarized in Figure 1.1 are no longer limited to patients with grades 1 and 2 hypertension, but also extend to subjects with high normal blood pressure. They also describe in greater detail how to deal with patients with grade 3 hypertension. Consideration of subjects with systolic blood pressure 130$139 \mathrm{mmHg}$ and diastolic blood pressure $85-89 \mathrm{mmHg}$ for possible initiation of antihypertensive treatment is based on the following recent evidence: 1 . The PROGRESS study (14) has shown that patients with previous stroke or transient ischaemic attack and blood pressures $<140 / 90 \mathrm{mmHg}$, if left untreated, have an incidence of cardio vascular events of about $17 \%$ in 4 years

Figure 1.1 (adapted from ESH guidelines)

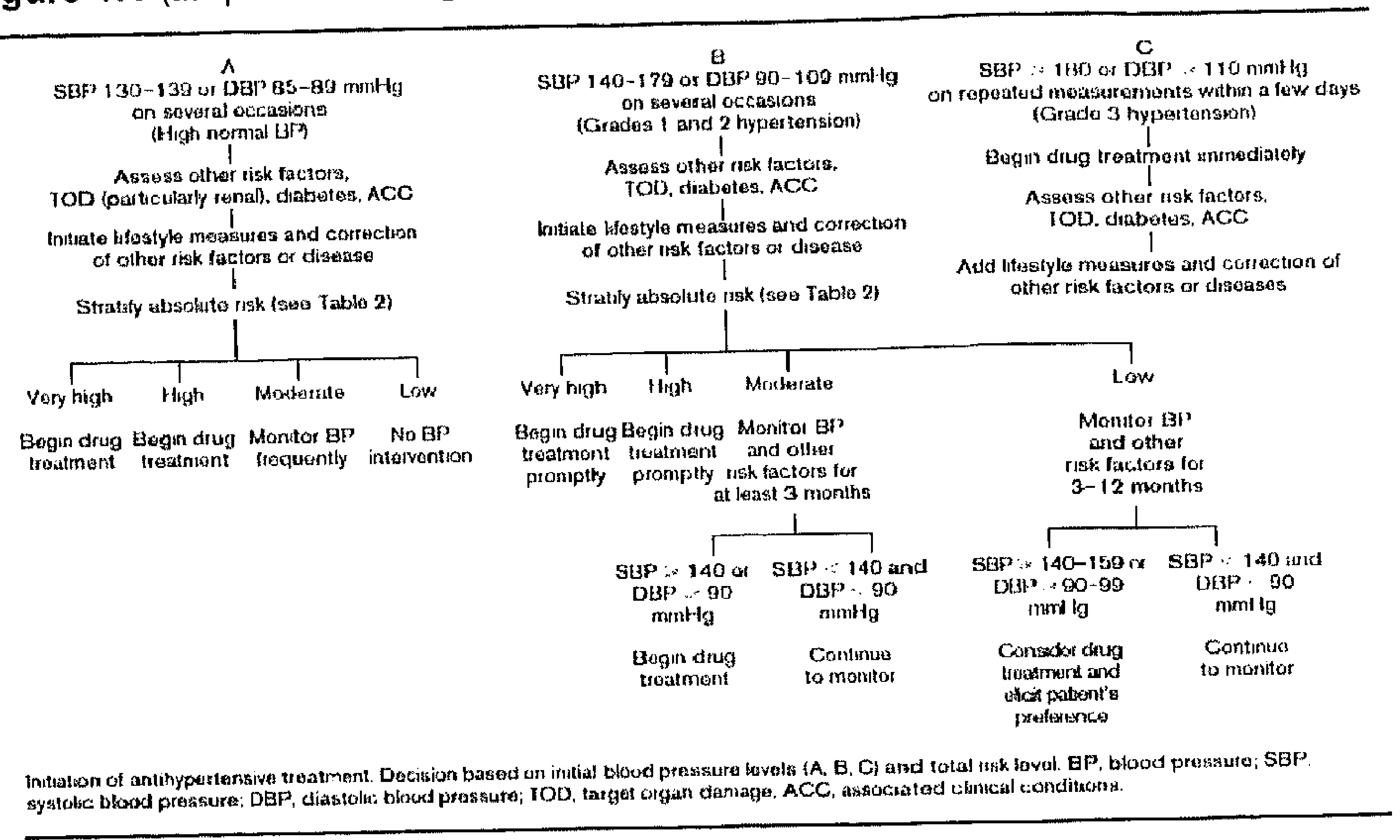

(very high risk according to the guidelines), and their risk is decreased by $24 \%$ by blood pressure lowering. 2. Similar observations have been made in the HOPE study (15) for 'normotensive' patients with high coronary risk. 3. The ABCD-Normotensive trial (16) has shown that type 2 diabetic patients with blood pressures $<140 / 90 \mathrm{mmHg}$ may also benefit by more aggressive blood pressure lowering, at least for stroke prevention and progression of proteinuria. 4. The Framingham Heart Study (10) has 
shown that male subjects with high normal blood pressure have a 10-year cardiovascular disease incidence of $10 \%$, i.e. in the range that these guidelines classify as low added risk. 5. The recent MONICA trial has demonstrated that progressing from optimal or normal blood pressure to high-normal blood pressure or hypertension carries the same risk as sustained hypertension (17).

\section{Goals of treatment}

The primary goal of treatment of the hypertensive patient is to achieve the maximum reduction in the long-term total risk of cardiovascular morbidity and mortality. This requires treatment of all the reversible risk factors identified, including smoking, dyslipidaemia or diabetes, and the appropriate management of associated clinical conditions, as well as treatment of the raised blood pressure per se (1).

\section{How to initiate treatment?}

The current treatment strategies are based on life style changes and continuous antihypertensive treatment. Lifestyle measures should be instituted whenever appropriate in all patients, including subjects with high normal blood pressure and patients who require drug treatment (13). The purpose of life style changes is to lower blood pressure and to control other risk factors and clinical conditions present. The lifestyle measures that are widely agreed to lower blood pressure or cardiovascular risk, and that should be considered in all patients, are: (1) smoking cessation; (2) weight reduction; (3) reduction of excessive alcohol intake; (4) physical exercise; (5) reduction of salt intake; and (6) increase in fruit and vegetable intake and decrease in saturated and total fat intake.

Smoking cessation is probably the single most powerful lifestyle measure for the prevention of non-cardio-vascular and cardiovascular diseases, including stroke and coronary heart disease (18). Although any independent chronic effect of smoking on blood pressure is small (19) and smoking cessation does not lower blood pressure (20), total cardiovascular risk is greatly increased by smoking (18).

There is a linear relationship between alcohol consumption, blood pressure levels and the prevalence of hypertension in populations. Beyond that, high levels of alcohol consumption are associated with a high risk of stroke (21). 
Excess body fat predisposes to raised blood pressure and hypertension (22). Weight reduction reduces blood pressure in overweight patients and has beneficial effects on associated risk factors such as insulin resistance, diabetes, hyperlipidaemia and left ventricular hypertrophy. The blood-pressure-lowering effect of weight reduction may be enhanced by a simultaneous increase in physical exercise (23), by alcohol moderation in overweight drinkers (24) and by reduction in sodium intake (25). Physical fitness is a rather strong predictor of cardiovascular mortality independent of blood pressure and other risk factors (26).

Epidemiological studies suggest that dietary salt intake is a contributor to blood pressure elevation and to the prevalence of hypertension (27). Randomized controlled trials in hypertensive patients indicate that reducing sodium intake by $80-100 \mathrm{mmol}$ $(4.7-5.8 \mathrm{~g})$ per day from an initial intake of around $180 \mathrm{mmol}(10.5 \mathrm{~g})$ per day will reduce blood pressure by an average of $4-6 \mathrm{mmHg}(28)$ or even more if combined with other dietary counselling (29), and enhance the blood-pressure-lowering effect of medication.

\section{Antihypertensive treatment}

Recommendations about pharmacological therapy are preceded by analysis of the available evidence (as provided by large randomized trials based on fatal and non-fatal events) of the benefits obtained by antihypertensive therapy and of the comparative benefits obtained by the various classes of agents. This is the strongest type of evidence available. Most of these outcome trials have been subjected to metaanalyses, either to arrive at more precise and generalizable conclusions, or to answer questions on subgroups, which could not be addressed in individual studies (12). Table 1.3 summarizes the results of meta-analyses of trials performed in mostly systolic-diastolic hypertension $(30,31)$ and in elderly patients with isolated systolic hypertension (32). Antihypertensive treatment resulted in significant and similar reductions of cardiovascular and all-cause mortality in both types of hypertension. With regard to cause-specific mortality, Collins et al. (30) observed a significant reduction in fatal stroke $(45 \% ; P<0.001)$, but not in fatal coronary heart disease $(11 \% ; N S)$. This could be related to age because coronary mortality was significantly reduced by $26 \%$ $(P<0.01)$ in a meta-analysis on elderly with systolic-diastolic hypertension (33). Fatal and non-fatal strokes combined, and all coronary events, were significantly reduced in 
the two types of hypertension. The evidence based drug choice is a rather complex field. As it does not reflect the main goal of this thesis I will not describe the drug groups. According to the baseline blood pressure and the presence or absence of complications, it appears reasonable to initiate therapy either with a low dose of a single agent or with a low dose combination of two agents. Initiation of treatment by combination therapy was effectively

Table 1.3 (adapted from ESH guidelines)

\begin{tabular}{|c|c|c|c|c|}
\hline & \multicolumn{2}{|c|}{ Syetolu-diastolic hypertansicn } & \multicolumn{2}{|c|}{ Ecolated syctolic hypertension } \\
\hline & Rigk reduction & $P$ & Risk redututian & $p$ \\
\hline \multicolumn{5}{|l|}{ Mortelity } \\
\hline als cauge & -144 & $\because 0.01$ & -130 & 0.02 \\
\hline $\begin{array}{l}\text { cardiovascular } \\
\text { nonwcardioyagcutar }\end{array}$ & $\begin{array}{r}-210 \\
-10\end{array}$ & $=0.001$ & -188 & $\begin{array}{l}0.01 \\
\text { MS }\end{array}$ \\
\hline \multicolumn{5}{|l|}{ Fatal and non. fetal Events } \\
\hline strcke & $-42 \%$ & $\therefore 0.001$ & $-30 \%$ & $\therefore 0.001$ \\
\hline coronary & $-140 \%$ & $=0.01$ & $-23 \%$ & $\approx 0.001$ \\
\hline
\end{tabular}

tested in the VA study at the beginning of the antihypertensive treatment trial era (34, 35 ) and recently in the PROGRESS studies (14). If low-dose monotherapy is chosen and blood pressure control is not achieved, the next step is to switch to a low dose of a different agent, or to increase the dose of the first compound chosen (with a greater possibility of causing adverse effects) or to move to combination therapy. If therapy has been initiated by a low-dose combination, a higher dose combination can subsequently be used or a low dose of a third compound added. An obvious disadvantage of initiating with two drugs, even if at a low dose, is that of potentially exposing the patient to an unnecessary agent, but the advantages are that: (1) by using two drugs with different mechanisms of action, it is more likely that blood pressure and its complications are controlled; (2) by using combinations, both the first and second drugs can be given in the low dose range that is more likely to be free of side-effects; (3) fixed low-dose combinations are available in Europe as well as in other parts of the world, allowing the administration of two agents within a single tablet, thus optimizing compliance. The following two-drug combinations have been found to be effective and well tolerated (Figure 1.2): 
-diuretic and $\beta$-blocker;

- diuretic and ACE inhibitor or angiotensin receptor antagonist;

-calcium antagonist (dihydropyridine) and $\beta$-blocker;

-calcium antagonist and ACE inhibitor or angiotensin receptor antagonist;

-calcium antagonist and diuretic;

- $\alpha$-blocker and $\beta$-blocker;

-other combinations (e.g. with central agents, including a2-adrenoreceptor agonists and imidazoline 12 receptor modulators, or between ACE inhibitors and angiotensin receptor antagonists) can be used if necessary, and three or four drugs may be required in many cases.

\section{Current effectiveness of treatment strategies}

Despite the options of lifestyle modifications and antihypertensive drugs which effectively lower blood pressure and cardiovascular risk the outcome remains low (11). The awareness and appropriate management of hypertension among hypertensive patients in the USA remain low: $31 \%$ were not aware of their disease, only two thirds $(66 \%)$ were told by health professionals to adopt lifestyle modifications or take drugs to control hypertension, and only $31 \%$ controlled their hypertension.

Figure 1.2: (adapted from ESH guidelines)

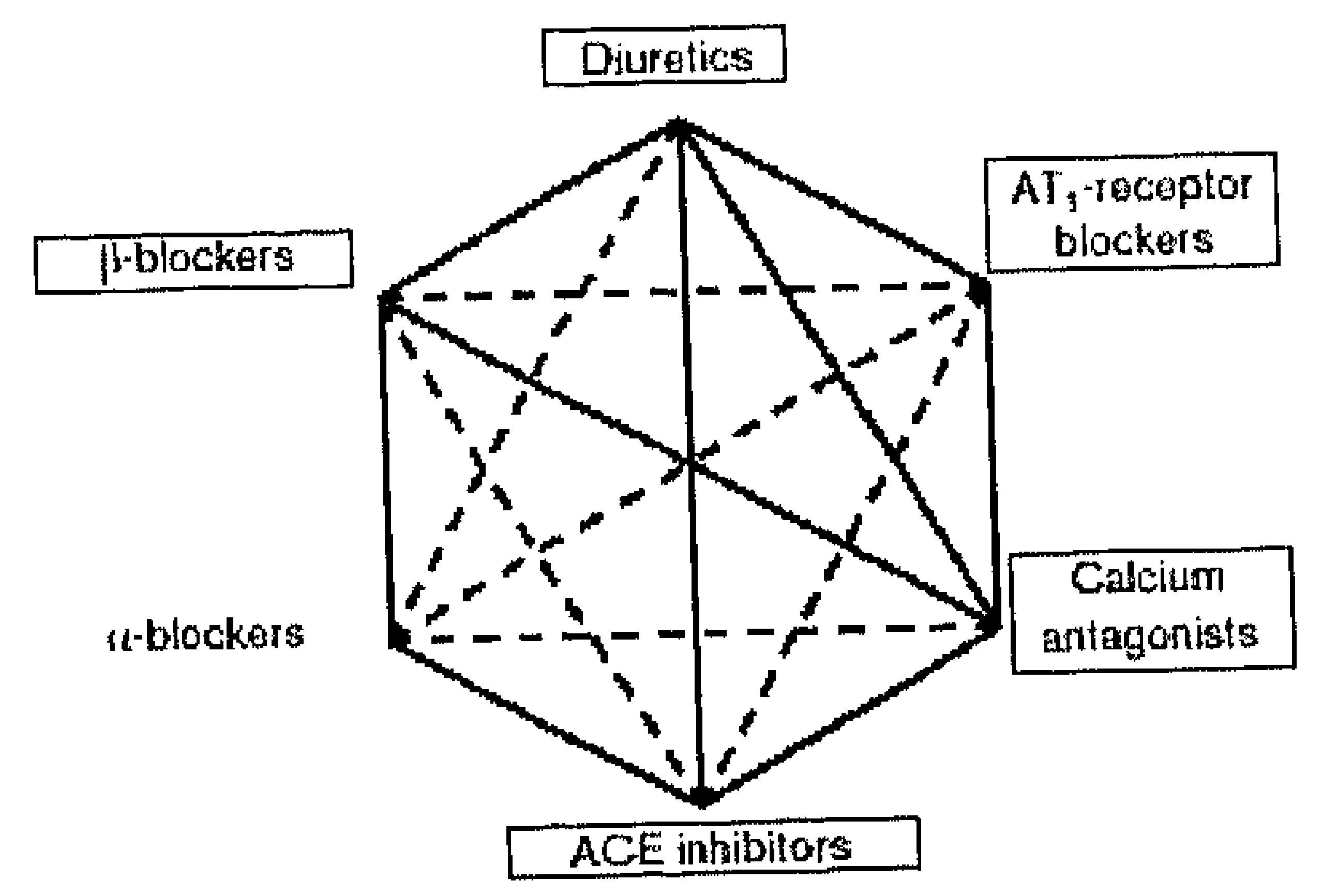

Postible combinations of difereat classes of antihypertensiva agents The most rational combinations ore represenled as thick lines. ACE. angiotensin-corwerting anzyme. The Irames indicate clazses of antihypentensive agents proven to be beneficial in controlled interventional trials. 
These devastating results are not restricted to the United States. This epidemiological phenomenon is also apparent in Europe (36). Thus, generalizing that only one third of the hypertensive world population is properly treated one billion remain not properly treated. Furthermore, approximately 1.5 billion individuals exist with high normal blood pressure and according to the MONICA trial the same cardiovascular risk as sustained hypertension. Therefore, more than 2.5 billion people with elevated blood pressure and cardiovascular risk are not effectively treated or untreated.

\section{The costs of therapeutic strategies}

Treatment and complications among the 50-60 millions of hypertensive people in the United States are estimated to cost $\$ 37$ billion annually (37). Antihypertensive drug costs alone account for $\$ 15.5$ billion per year, the other $\$ 21.5$ billion per year account for the treatment of complications. Taking into account that, according to the cardiovascular risk, all hypertensive and prehypertensive people (United States: 50-60 millions) should be treated, but only two third of the hypertensive people are treated, one might expect extra costs for antihypertensive medication of approximately $\$ 31$ billion per year only for the United States.

A population-based survey on cardiovascular disease determined risk factors in the Netherlands from 1996 to 2002 (1). A total of 10820 men and women, aged 30-59 years, were included in the study. The main outcome measures of the study were: Prevalence of hypertension, treatment, and control of hypertension, and determinants of undertreatment of hypertension. Hypertension was defined as: systolic blood pressure (SBP) $>140 \mathrm{mmHg}$ and/or diastolic blood pressure (DBP) $>90 \mathrm{mmHg}$, and/or the use of antihypertensive medication. The prevalence of hypertension in men was $21.4 \%$ and in women $14.9 \%$, and $17.9 \%$ of the hypertensive men and $38.5 \%$ of the hypertensive women were receiving antihypertensive medication. Of the untreated hypertensives, $21.9 \%$ of the men and $13.6 \%$ of the women were eligible for treatment with antihypertensive medication according to Dutch guidelines. Female gender and the use of cholesterol-lowering medication were associated with an increased chance of being treated. Subjects who were physically active, on a low salt diet, and current smokers had an increased chance of being untreated. Taking cholesterol-lowering medication and no asthma or allergy were factors associated with better control of 
blood pressure. In conclusion, a considerable proportion of hypertensives were untreated and uncontrolled $(38,39)$.

Although under these circumstances the costs related to complications might decrease, an absolute excess in costs must be expected with common continuous antihypertensive treatment of all subjects at risk (hypertensives and prehypertensives). As this does not seem to be socioeconomically feasible, other additive treatment strategies are warranted which lower cardiovascular morbidity and mortality without producing high antihypertensive drug costs.

This raises the crucial question whether we need to adapt therapeutic strategies searching for alternatives which increase the outcome and reduce cardiovascular morbidity and mortality. Furthermore, the additive strategy must be socioeconomically feasible.

\section{General thoughts regarding therapeutic concepts}

The general question arises where the additive therapeutic strategy could derive from. Life style changes represent the most basal option to influence the outcome, as previously discussed. In contrast, continuous antihypertensive medication does not cure hypertension and related cardiovascular morbidity and mortality (40). Medication is able to decrease blood pressure and target organ damage thereby reducing cardiovascular morbidity and mortality. However, medication starts at a time point in life at which cardiovascular alterations are already apparent (41).

Medication is based on targeting several pathomechanisms involved in elevated blood pressure and target organ damage, counteracting them and thereby delaying clinically relevant disorders, but none of the treatments aims at the cause of hypertension.

If we hypothesize that hypertension is an active disease with a cause and an individual start point, the optimal treatment strategy would be to attack the cause of the development of hypertension prior to the starting point, thereby curing hypertension and extincting hypertension as a cardiovascular risk factor. For this ideal situation it is necessary to understand in more detail the development of hypertension and the underlying pathomechanisms. In this respect it is of interest that most theories 
discussing potential mechanisms are several decades old, and are related to the kidney.

\section{Role of the kidney in hypertension in humans}

The concept of a primary renovascular disease causing essential hypertension

While it was known that patients with primary hypertension often did not have evidence of renal insufficiency or end-stage disease at autopsy, the possibility that hypertension may still reflect an intrarenal disease process was introduced by Goldblatt (42). Both noted that intrarenal arteriolar disease was present in the majority of patients with hypertension. These authors suggested that a primary thickening of arterioles within the kidney could lead to intrarenal ischemia, and that a compensatory response would be an increase in blood pressure. To test this hypothesis, Goldblattt partially occluded the renal artery of dogs and documented a rapid increase in blood pressure (43). While recognizing that renal artery stenosis was not the same as intrarenal vascular disease, Goldblatt nevertheless suggested that both processes resulted in renal ischemia, which was central to the pathogenesis of hypertension (42). Although Goldblatt proposed (43) that primary disease involving the renal arterioles was the cause of essential hypertension, neither could provide a mechanism by which it could develop.

The hypothesis of Goldblatt was largely supported by histological data. In the classic autopsy study by Moritz and Oldt (44), renal arteriolar stenosis was documented in $97 \%$ of individuals dying from hypertension but in only $12 \%$ of non-hypertensive controls. Furthermore, the observation in the same study that arteriolar changes in other organs such as spleen did not correlate with the presence of hypertension suggested that the disease within the kidney was critical in order for hypertension to develop. Additional support came from studies reported by Castleman and Smithwick (45). During their ill-fated attempts to control hypertension by thoracolumbar sympathectomy, they obtained renal biopsies in over 1300 cases, which, unlike the autopsy study, included patients at different stages of the natural history of hypertension $(45,46)$. The vast majority $(98 \%)$ of patients with hypertension were found to have significant renal arteriolar disease at sympathectomy (46). 
Although the great majority of patients with hypertension had renal arteriolar disease that could be documented by biopsy or autopsy, there were occasional hypertensive patients in who no renal arteriolar disease could be identified (44-46). One possibility proposed by Goldblatt (42) was that this simply reflected a sampling error. Another possibility suggested by Sommers (46) was that early hypertension might be mediated more by renal arteriolar spasm, and that the structural changes would occur later. In support of this, they reported that ischemic changes in the tubules, such as dilatation and atrophy, were not only present in patients with renal arteriolar disease but also in $50 \%$ of hypertensive patients with no evidence of arteriolar disease, and were the most sensitive markers for hypertension (46).

Natural history studies performed prior to the availability of effective antihypertensive agents reported that as many as $40 \%$ of hypertensive patients eventually developed proteinuria and 10-20\% developed renal insufficiency (47). Thus the concept of intrinsic renal disease as the cause of hypertension was largely discarded. Indeed, renal parenchymal causes of hypertension are considered separately from essential hypertension, and are thought to contribute to only $5 \%$ of all cases (48).

\section{Primary renal abnormalities in hypertension}

In spite of the mounting evidence that renal pathological injury was the consequence and not the cause of essential hypertension, Guyton concluded, based on physiological principles and mathematical modeling that the kidney had to be the source of persistent hypertension (49). Borst (50) and Guyton (49) further suggested that the physiological abnormality must involve a relative defect in sodium excretion in response to alterations in blood pressure. The kidney is considered to be a filter and for each given value of sodium intake (equal, in steady-state and normal conditions, to urinary sodium output), a given steady mean blood pressure is achieved (51). As hypertension develops, the renal filter is altered and a higher mean blood pressure is required to maintain sodium balance, as under normal conditions. Thus there is a shift of the pressure-diuresis curve toward higher blood pressure values. Different patterns of pressure-diuresis curves have been described according to different etiologic profiles of hypertensive disorders (hyper-activity of the renin-angiotensin system, abnormal sodium balance, etc) (49). 
Within the organism, the pressure-diuresis mechanism is achieved through a negative feedback loop that is defined by equations (with their corresponding specific coefficients), which characterize the resulting changes in fluid volumes, cardiac output, vascular resistance, and compliance (49).

Additional evidence for a renal defect in primary hypertension was provided by experimental and clinical renal transplantation studies (53). These data were found in animal experiments of Bianchi and Rettig $(54,55)$. In the mid 1980s, 3 studies on renal transplant patients were published that supported a major role for the kidney in human essential hypertension. The classical study by Curtis et al. (56) demonstrated the sustained remission of essential hypertension in 6 patients who had received kidney transplants from normotensive donors. Two other studies took the opposite approach by showing that grafts from donors with presumed hypertension or from donors with known family background of essential hypertension (57) caused more severe hypertension and the need for more rigorous antihypertensive treatment in caused more severe hypertension and the need for more rigorous antihypertensive treatment in the recipients. The results of one of these studies (57) were later confirmed by a long-term follow-up (58).

Figure 1.3: Pressure-natriuresis curve

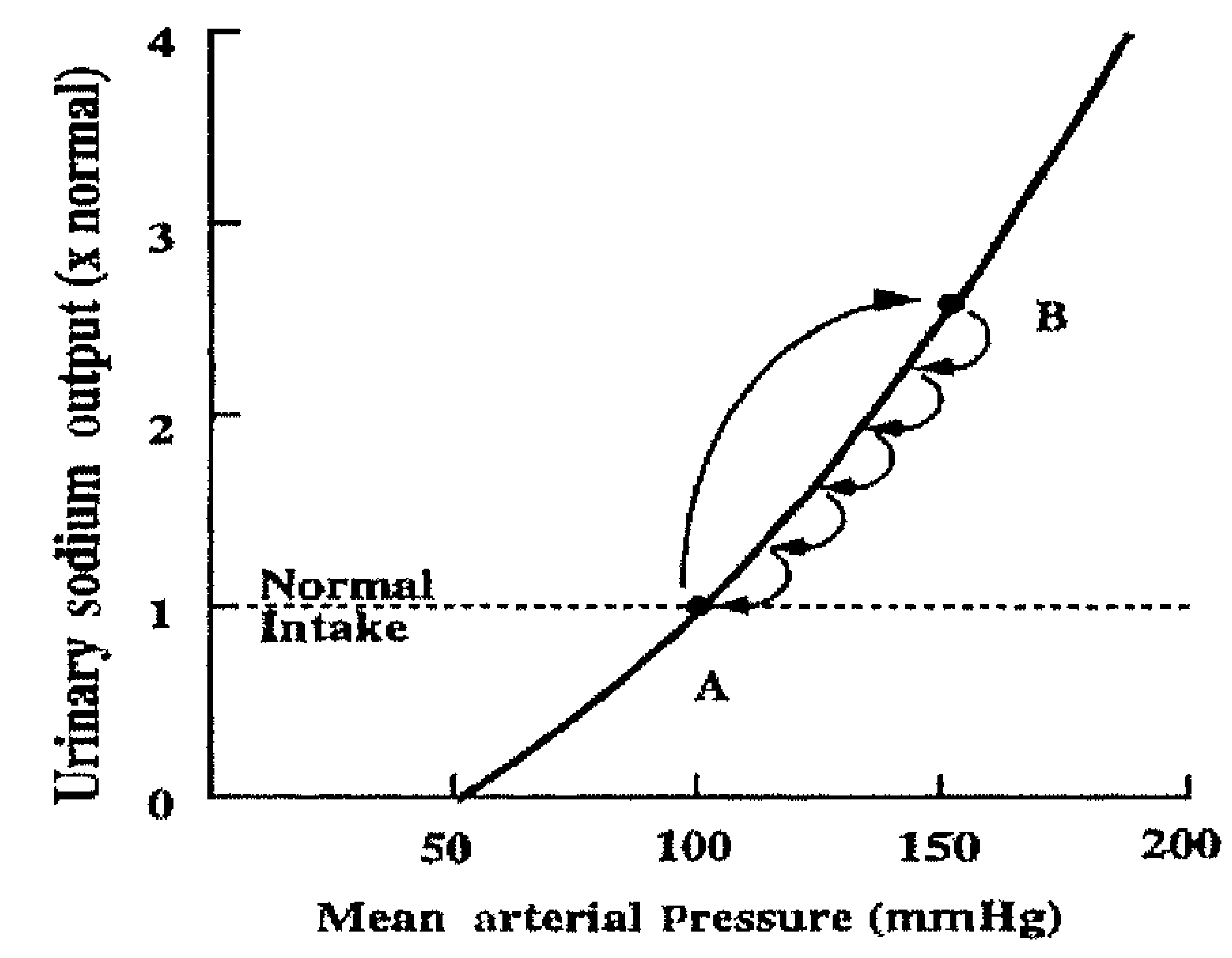

The diagram demonstrates the dynamic relation between urinary sodium and mean arterial pressure (Original: Hall et al. (52)) 
A commonly held hypothesis then developed that the abnormality in essential hypertension was primarily a physiological one, and that it involved a relative defect in sodium excretion. The observation that certain rare types of hereditary hypertension were associated with genetic mutations leading to increased sodium reabsorption fuelled this concept (59). However no specific defect has been found that can account for the increased blood pressure observed in the majority of patients with essential hypertension.

\section{Role of renal vasculature}

In the 1970's differences in the structurally determined pre-and postglomerular reistance ratio were estimated by comparing the respective relationships between arterial perfusion pressure ( $\mathrm{Pa}$ ) and glomerular filtration rate (GFR) (60). Folkow et al. demonstrated that in 5 weeks old SHR and age-matched normotensive controls, the total renal vascular resistance was similar. In contrast, in adult SHR the Pa-GFR curve was displaced but the curve steepness was still largely similar (61). Folkow suggested from these data that in SHR a structurally based vascular narrowing, which in the kidney could reflect the preglomerular arterioles, is compatible with this pattern (62). Histological studies demonstrated later that already young SHR have abnormalities in the renal microcirculation (63-65) and a reduced nephron number (66) before hypertension has established in these animals. Comparable observations were made in primates (67) and hypertensive humans (68). The abnormalities can be summarized as renovascular wall thickening and hypercontractile reactivity which precedes hypertension in SHR. These results were confirmed in the human (69).

The Dutch Hypertension and Offspring Study investigated renal hemodynamics in normotensive subjects with hypertensive and normotensive parents (70). This study found that renal blood flow is lower and plasma renin and aldosterone concentrations are suppressed in the children of hypertensive parents, as compared with the children of normotensive parents. They suggested that changes in the renal hemodynamics take place at an early stage in the development of familial hypertension. These changes may set the stage for a more rapid and pronounced increase of blood pressure in the children of hypertensive parents. In a follow-up study the same group furthermore observed that the children of hypertensive parents also demonstrate increased left ventricular mass (71). Thus, evidence exists that renal hemodynamics 
are altered in hypertensive animal models and hypertensive humans. Furthermore these aspects are already apparent at an early stage of the development of hypertension.

\section{Renal ischemia revisited in animal models}

In a more detailed analysis of the renal microcirculation the role of medullary ischemia was further emphasized. Renal blood flow measurements suggested that the decrease in renal blood flow need not be to the entire kidney but rather could be restricted to the medulla (72). In the spontaneously hypertensive rat, increased afferent arteriolar tone that led to a reduction in papillary blood flow could be documented, and blocking vasoconstriction (with angiotensin-l-converting enzyme inhibitors) or stimulating vasodilation (with L-Arginine, which stimulates nitric oxide production) could prevent the development of hypertension (73). In addition, in the Dahl salt-sensitive rat, hypertension was associated with decreased renal medullary blood flow, and both could be prevented by the intramedullary infusion of L-arginine (74). Furthermore, normal rats could be made hypertensive by the intramedullary infusion of inhibitors of the nitric oxide system or with antisense oligonucleotides to neural nitric oxide synthase, which is one of its synthesizing enzymes (75).

\section{Salt sensitivity in animal models}

Salt sensitivity can be induced in normal rats by measures that induce subtle renal injury. Short-term administration of angiotensin II, catecholamines, L-NAME, which inhibits nitric oxide, or cyclosporin leads to the development of salt -sensitive hypertension that can be documented after the agent has been discontinued (76-78). Acquired renal damage may also be important in genetic models of hypertension. For example, subtle renal injury can be documented in the prehypertensive Dahl saltsensitive rat and may predispose the animal to persistent hypertension There is also evidence that the renal inflammatory changes that occur in the spontaneously hypertensive rat may mediate salt sensitivity (76).

The mechanism for renal injury is likely to involve renal ischemia as most of these models are associated with vasoconstriction $(79,80)$. Renal injury may also be mediated by increased vascular reactivity, with sudden changes in blood pressure that 
are transmitted to the renal microvasculature due to an inadequate renal autoregulatory response (81). The histological changes primarily involve the microvasculature and tubulointerstitial compartments. There is evidence for mononuclear cell infiltration, low-grade interstitial fibrosis and arteriolosclerosis and capillary rarefaction $(77,82)$. Interestingly, these histological changes are similar to those observed in humans with essential hypertension $(44,45,83)$.

\section{SHR as an animal model to investigate the development of hypertension}

Taking the similarities of human and rat pathophysiology together, rat models of hypertension, in particular the SHR, seems to be a good model to investigate the development of hypertension. This is further strengthened by the fact that the SHR develops hypertension reproducibly between week 4-10 of age (84). Thus the problem in the human setting, where we do not know the onset of hypertension can be overcome in the rat model.

The development of hypertension in SHR is characterized by several aspects which are predominantly related to renal physiology. Evenwel et al. demonstrated an increase of cardiac output in 5 weeks old SHR when compared to age-matched WKY (2). This increased flow was mainly transmitted to the muscular and skin vascular bed. The renal vascular resistance was increased significantly already in 5 weeks old SHR. In 7-8 weeks old animals, cardiac output was not significantly different in SHR and WKY The increase in blood pressure was then characterized by a generalized increase in vascular resistance in almost all tissues. They concluded that the early increase in cardiac output in SHR is related to an increased central intravascular volume, possibly because of renal fluid retention (85).

Regarding the structural renovascular changes Skov et al. (3) showed that the lumen diameter of afferent arterioles close to the glomeruli showed a $17 \%$ reduction in young spontaneously hypertensive rats $(15.4+/-0.6 \mu \mathrm{m})$ compared with Wistar-Kyoto rat arterioles $(18.5+/-0.3 \mu \mathrm{m}, \mathrm{p}<0.001)$. However, this was not due to media hypertrophy, because media cross-sectional area was smaller $(p<0.001)$ in spontaneously hypertensive rats $\left(210+/-6 \mu \mathrm{m}^{2}\right)$ compared with Wistar-Kyoto rats (274 $+1-16 \mu \mathrm{m}^{2}$ ). They concluded that the lumen reduction in renal afferent arterioles in spontaneously hypertensive rats is not the result of an encroachment on the lumen by a hypertrophic media (64). An intervention on these structural abnormalities is possible 
with ACE-inhibitor and ARBs. Both reduce media thickness and media to lumen ration and increase lumen diameter (63).

The maintenance of hypertension in adults can be influenced by the level of dietary sodium intake. Adult SHR given an increased sodium load in their drinking water developed a higher blood pressure than SHR maintained on regular sodium diet (86). This is also seen in young SHR where it is associated with elevated plasma norepinephrine concentrations $(87,88)$. In contrast sodium restriction leads to a persistent reduction of blood pressure and, if performed after weaning, to a reduced development of hypertension $(89,90)$. The blood pressure effect is associated with a reduction of body weight. Reduction of regular food intake without alterations in sodium intake further demonstrated a reduced blood pressure $(91,92)$. Directly related to sodium and food intake is the body fluid composition. The body fluid volume is known to be increased in young SHR (93). This is driven by an increased extracellular fluid in SHR, while the plasma amount is similar in SHR and normotensive rats. The extended volume of extracellular fluid can be reduced by the aldosterone receptor antagonist spironolactone (94). This is related to the finding that aldosterone is increased (95) and expression of angiotensin receptors is enhanced in young SHR as compared to WKY (96).

An overreactivity of the renin-angiotensin-aldosterone system (RAAS) has been documented in young SHR, coinciding with the period of rapid blood pressure rise (97). This overreactivity is seen in an increased renal resistance and tubuloglomerular feedback. Both characteristics can be attenuated by inhibiton of the RAAS (98). An additional option to counteract the characteristics is to inhibit actions of thromboxane and other vasoconstrictor cyclooxygenase products $(99,100)$. This is furthermore in line with the observation of Sacerdoti that tin given during the early phase of hypertension can attenuate blood pressure development (101) as it antagonizes cytochrome $\mathrm{P} 450$ pathways involved in the production of contractile elements (102).

\section{Transient prehypertensive treatment in SHR}

Already in the 1980s several authors described an option to attenuate the development of hypertension in the SHR by a transient drug treatment during a time window early in life (4-10 weeks of age) which coincides with the steepest blood pressure increase in $\operatorname{SHR}(84,103)$. Therefore this period was also called 
prehypertensive. Transient prehypertensive drug treatment leading to an attenuated development of hypertension is largely related to the renin-angiotensin system. The underlying mechanism remains uncertain, although the kidney is thought to be involved in the effects $(104,105)$. Prolonged blood pressure attenuation is described with ACE-inhibitors $(84,103,106-116)$ and ARBs $(91,92)$ (Table 1.4).

Table 1.4 Summary of studies dealing with transient prehypertensive treatment

\begin{tabular}{|c|c|}
\hline Transient prehypertensive Treatment & Effective \\
\hline \multicolumn{2}{|l|}{ Transient prehypertensive ACE-inhibition } \\
\hline Berecek, K. H., Nagahama, S., and Oparil, S. (1984) (103) & YES \\
\hline Wu, J. N., Edwards, D., and Berecek, K. H. (1994) (109) & YES \\
\hline $\begin{array}{l}\text { Harrap, S. B., Van der Merwe, W. M., Griffin, S. A., Macpherson, F., and Lever, A. F. } \\
(1990)(111)\end{array}$ & YES \\
\hline Harrap, S. B., Mirakian, C., Datodi, S. R., and Lever, A. F. (1994) (84) & YES \\
\hline O'Sullivan, J. B., and Harrap, S. B. (1995) (114) & YES \\
\hline Kost, C. K., Jr., Li, P., and Jackson, E. K. (1995) (116) & YES \\
\hline Regan C.P., Bishop, S. P., and Berecek, K. H. (1997) (108) & YES \\
\hline O'Suilivan, J. B., and Harrap, S. B. (1999) (115) & YES \\
\hline \multicolumn{2}{|l|}{ Transient prehypertensive angiotensin receptor blockade } \\
\hline Bergstrom, G., Johansson, I., Wickman, A., Gan, L., and Thorup, C. (2002) (104) & YES \\
\hline Nakaya, H., Sasamura, H., Hayashi, M., and Saruta, T. (2001) (105) & YES \\
\hline Pollock, D. M., and Morsing, P. (1999) (120) & YES \\
\hline \multicolumn{2}{|l|}{ Transient prehypertensive calcium channel blockade } \\
\hline $\begin{array}{l}\text { Rizzoni, D., Castellano, M., Porteri, E., Bettoni, G., Muiesan, M. L., and Agabiti Rosei, E. } \\
(1994)(117)\end{array}$ & YES \\
\hline Christensen, K. L., Jespersen, L. T., and Mulvany, M. J. (1989) (118) & NO \\
\hline \multicolumn{2}{|l|}{$\begin{array}{l}\text { Transient prehypertensive beta-blocker use, vasodilator treatment, endothelin } \\
\text { antagonism, tin treatment }\end{array}$} \\
\hline Christensen, K. L., Jespersen, L. T., and Mulvany, M. J. (1989) (118) & NO \\
\hline Li, J. S., and Schiffrin, E. L. (1995) (119) & NO \\
\hline $\begin{array}{l}\text { Sacerdoti, D., Escalante, B., Abraham, N. G., McGiff, J. C., Levere, R. D., and } \\
\text { Schwartzman, M. L. (1989) (101) }\end{array}$ & YES \\
\hline
\end{tabular}


One study by Rizzoni additionally describes the blood pressure lowering effects of a calcium-antagonist which is transiently administered prehypertensively (117). This could not be confirmed by Christensen (118). Beta-blockers, vasodilators and endothelin antagonists are ineffective in this treatment scheme $(118,119)$. Recently, the Trial of Preventing Hypertension (TROPHY) applied this treatment strategy for the first time in humans (121). The study was based on a two years treatment of prehypertensive individuals with an ARB and thereafter drug withdrawal and another two years period of observation. The trial demonstrated that the treatment strategy is not harmful to the prehypertensive individuals. Additionally the authors described a risk reduction of $15 \%$ to develop new-onset hypertension at the end of the observation period which reflects a time point 2 years after drug withdrawal. Therefore, for the first time evidence was presented that transient prehypertensive treatment is not only feasible in rats but also in humans. This further increases the need to answer relevant aspects which until now remained uncertain in transient prehypertensive treatment, in particular as definition of the blood pressure parameters in TROPHY received criticism $(122,123)$.

Although blood pressure follow-up in rats was maintained up to adulthood it remains uncertain how long a blood pressure reduction is apparent (105). This is in particular relevant as in the SHR and the hypertensive human individual hypertension blood pressure increase is a continuous process with slowly accumulating target organ damage which becomes clinically relevant mainly at later stage in life. In this respect it remains uncertain in how far transient prehypertensive treatment leads to a protection of the target organs and whether this is apparent up to a clinically relevant age. Moreover, it is fully unclear whether, beside the blood pressure lowering, other blood pressure independent effects could contribute to the beneficial effect of transient prehypertensive treatment. And after investigation of transient prehypertensive treatment in the animal model it remains uncertain how to transfer the strategy to the human situation. To which time point in life is the prehypertensive time window in SHR comparable? Can we individually predict who will develop new-onset hypertension in the near future? 


\section{Choice of drugs in early development of hypertension}

As demonstrated in Table 1.4 the majority of animal experiments investigated ACEinhibitors and ARB. We furthermore emphasized on the last pages the role of the RAAS during the development of hypertension. However, other investigators focused on the blood pressure lowering, largely excluding the mechanism of prehypertensive treatment as well as their long-term effects of the treatment. To assess these factors, we chose for deeper analyses an ARB as main drug of interest as it represents the most downstream drug in the RAS so far analyzed. We extended our drug spectrum by additionally using a mineralocorticoid receptor antagonist to assess the role of aldosterone which is closely linked to the RAS and involved in the development of hypertension.

\section{Aims of the thesis}

1. To investigate how long blood pressure reduction is apparent after transient prehypertensive treatment in SHR.

2. To investigate in how far target organ damage is ameliorated by the transient prehypertensive treatment up to advanced age.

3. If target organ damage remains reduced, are the effects blood pressure dependent or independent?

4. What is the underlying mechanism of the beneficial effects of transient prehypertensive treatment?

5. Do markers exist in rats which correlate with the development of hypertension and can these markers be transferred into the human setting?

In the following chapters these question will be subsequently discussed and culminate in the discussion of the results including an outlook into the future of transient prehypertensive treatment in humans and ongoing studies directly based on the results of this thesis.

To reach the aims we used a set-up of transient prehypertensive treatment in young SHR during their second month of life. Based on this setting we performed pharmacological interventions investigating blood pressure and end organ structure and function. Blood pressure was investigated by using intraarterial catheters or for 
long-term assessment telemetry devices. Target organ damage was investigated longitudinally using non-invasive settings such as 24-hour urine collection and echocardiography. Invasive measurements are used before the rats are killed. Here we used flowmeter to assess renal blood flow and to calculate renal vascular resitance. A laser Doppler flowmeter was used to assess medullary blood flow. A Millar catheter was inserted into the left ventricle of the rat heart to assess cardiac contractility and relaxation under baseline conditions and during dobutamine infusion. To further assess renal medullary function we developed a kidney slice model to investigate vasa recta contraction. Target organ damage was also assessed using (immuno)histochemistry with subsequent semi-automized quantitative analyses. Results were confirmed by using standard molecular biology approaches as Western Blotting and quantitative PCR.

Chapter TWO deals with the long-term blood pressure reduction and cardiac and renal glomerular effects till advanced age. Chapter THREE selectively investigates the longterm effects on the vasculature. Chapter FOUR investigates in how far blood pressure independent effects are involved in the long-term effects of transient prehypertensive treatment. Chapter FIVE summarizes structural differences in the renal medulla between young prehypertensive SHR and normotensive controls. Chapter SIX demonstrates which role these medullary differences play in the transient prehypertensive treatment. Chapter SEVEN finally deals with the translational process of aspects determined in Chapter FIVE and SIX leading to a potential individual prediction of prehypertensive subjects who will develop new-onset hypertension in the near future. In chapter EIGHT the results are discussed, conclusions are drawn and future studies are indicated. 


\section{CHAPTER TWO:}

Transient AT1 receptor-inhibition in prehypertensive SHR results in maintained cardiac protection until advanced age

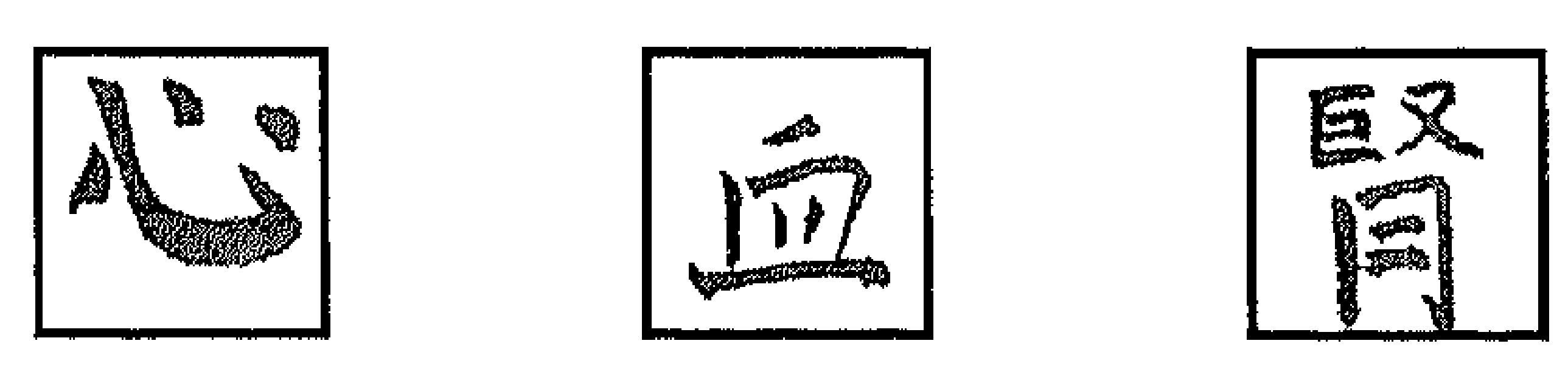

Marcus Baumann, Ben J.A. Janssen, J.J. Rob Hermans, Carine Peutz-Kootstra, Oliver Witzke, Jos F.M. Smits, Harry A.J. Struijker Boudier

J Hypertens. 2007; 25(1): 207-215 


\section{Abstract}

Objective: In young SHR transient angiotensin II type 1 receptor blockade (AT1RB) decreases blood pressure for a prolonged period. We tested the hypothesis that transient ATIRB in SHR leads to cardiac protection until advanced age.

Methods: WKY, SHR and transiently losartan-treated SHR (SHR-Los: $20 \mathrm{mg} . \mathrm{kg}^{-1} . \mathrm{d}^{-1}$; week 4-8 of age) were followed up until week 72 of age ( $n=9$ each group) including repeated echocardiographic, radiotelemetric investigations and $24-h$ urine collection. End point measurements comprised left ventricular (LV) function parameters, LV histomorphology and molecular biology (type I and III collagen, brain natriuretic peptide (BNP), AT1R mRNA) as well as renal morphology.

Results: Prehypertensive treatment with losartan, but not with the general vasodilator hydralazine, reduced blood pressure until 48 weeks of age. In untreated SHR enddiastolic volume (EDV) increased from week 36 and LV ejection fraction (EF) fell from week 48. In contrast, age-related changes in EDV and EF were comparable in SHRLos and WKY up to 60 weeks of age. At 72 weeks of age EF was reduced in SHR-Los,

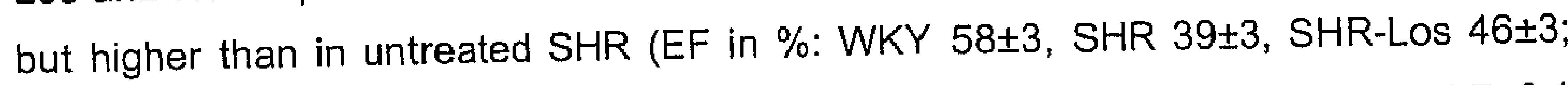
$P<0.01, P<0.05$, respectively). Heart weight to body weight ratio (SHR-Los $4.7 \pm 0.1$ $\mathrm{g} / \mathrm{kg}$; SHR $5.2 \pm 0.2 \mathrm{~g} / \mathrm{kg}$ ) and cardiac BNP mRNA levels were improved by treatment. LV histomorphology and 24-h albuminuria (in $\mathrm{mg} /$ day) were significantly improved in SHR-Los (SHR-Los $41 \pm 5$; SHR $80 \pm 22, P<0.05$ ).

Conclusions: In young SHR, transient AT1RB, attenuates the development of hypertension and exerts cardioprotective effects up to 72 weeks of age. 


\section{Introduction}

Long-lasting hypertension causes structural and functional cardiovascular modifications and increases associated morbidity and mortality (37). Myocardial remodeling in hypertensive hearts comprises left ventricular wall thickening, including myocyte hypertrophy and myocardial fibrosis (124). Both are adaptive mechanisms in response to left ventricular pressure overload and result in impaired pumping activity and myocardial stiffness of the heart (125).

The renin angiotensin system (RAS) is a major determinant of arterial pressure and volume homeostasis in mammals through the action of the vasoactive peptide angiotensin II on angiotensin II type 1 receptors (AT1R) (126). In the heart AT1Rs are expressed in cardiomyocytes, fibroblasts, coronary smooth muscle cells, and endothelial cells (126-128). Cardiac AT1Rs are upregulated in hypertension and cardiac hypertrophy (129). Pharmacological inhibition of the RAS $(130,131)$ as well as AT1R antisense treatment (107) induce in hypertensive rat models a long-lasting blood pressure decrease and attenuate the development of left ventricular hypertrophy. This leads to the view that blood pressure lowering is required to reach cardiac protection. Alternatively this may be a specific AT1R effect since treatment with AT1R antagonists have revealed additional effects on structural and functional remodeling and inflammatory processes (132).

To reach long-lasting cardiovascular protection continuous RAS antagonism is needed. This requires continuous pharmacological therapy linked with adverse effects (133) and high costs (134). A favorable therapeutic strategy should reach similar protective potential, without negative pharmacological and pecuniar side effects of continuous therapy. In this respect transient prehypertensive treatment is a potential therapeutic alternative. Early and transient ACE-inhibition $(103,111)$ and AT. antagonism (104) lead to prolonged blood pressure lowering at later ages. The recently published TROPHY trial in humans investigating transient AT1R antagonism in prehypertensive patients revealed similar results (121).

There are indications that the cardiac protection during adulthood is enhanced by this type of treatment in several experimental animal studies, too $(104,108)$. However, it remains unclear whether such protective effects persist into the late period of life when the majority of cardiovascular complications appear. Blood pressure monitoring was continued only up to 25 weeks of age in the aforementioned animal studies, which is 
not an advanced age considering the 1.5-2 years life span of hypertensive rats. Furthermore, in these studies $(104,111)$, only heart weight/body weight ratio was examined as a measure of cardiac morbidity. Thus it remains unknown at present whether prehypertensive treatment leads to cardioprotection into advanced age, a phase in which cardiovascular morbidity is highest.

The present study was designed to clarify whether transient prehypertensive AT1RB in young SHR exhibits cardioprotection till advanced age. We hypothesized that this is specifically mediated by AT1RB, and therefore included a subgroup of SHR which were treated with equipotent doses of the general vasodilator hydralazine. We longitudinally (up to 72 weeks of age) investigated WKY, SHR and prehypertensively losartan treated SHR with respect to blood pressure (radiotelemetry), heart (echocardiography) and renal function (albuminuria) completing the experiment with extended end point measurements including left ventricular function, histology and molecular biology.

\section{Methods}

One month old WKY and SHR treated with either saline $(n=21)$ or losartan $\left(20 \mathrm{mg} . \mathrm{kg}^{-}\right.$ ${ }^{1} . d^{-1}(n=21)$ ) or hydralazine (SHR-Hyd: $4 \mathrm{mg} \cdot \mathrm{kg}^{-1} \cdot \mathrm{d}^{-1} ; \mathrm{n}=11$ ) for four weeks using minipumps (Alzet 2004, Durect, CA, USA) that were implanted subcutaneously under isoflurane anaesthesia $(1.5 \%)$. All rats were housed under controlled conditions of temperature $\left(21^{\circ} \mathrm{C}\right)$ and light (12-hour light/dark cycle, $7 \mathrm{AM}$ to $\left.7 \mathrm{PM}\right)$ and were maintained on normal rat chow and water ad libitum. All experiments were approved by the animal ethics committee of the Maastricht University and performed in accordance with institutional guidelines.

In a first set of experiments we investigated if general antihypertensive treatment with hydralazine $(n=11)$ or AT1 receptor blockade losartan $(n=12)$ during the prehypertensive time frame (4-8 weeks of age) reduces blood pressure and heart weight four weeks after drug withdrawal as compared to untreated SHR $(n=12)$. Therefore we measured intra-arterial blood pressure in the conscious unrestrained state through a heparinized $(5 \mathrm{U} / \mathrm{ml})$ indwelling polyethylene catheter that was introduced into the left femoral artery two days before measurement at 8 and 12 weeks of age (135). At the end of this period we determined HW/BW ratio and interstitial 
collagen as histochemically detected with Sirius Red and quantified as described in more detail below.

In the second set of experiments each group consisted of 4 male and 5 female rats. The Dataquest IV telemetry system (Data Sciences International) was used for the measurement of systolic pressure, diastolic pressure, mean arterial pressure and heart rate as described previously (136). Two male and 3 female rats of each group received transmitter devices (TA11PA) at an age of 12 weeks. Hemodynamic data were obtained monthly for 48 hours sampling every minute for 10 seconds. Data acquisition started at 16 weeks of age and had to be stopped at 55 weeks of age due to failure of the batteries of the telemetry system.

Transthoracic echocardiography was performed in isoflurane-anesthetized rats of each group at three monthly intervals starting at week 24 and ending at week 72 as previously described (137). The end-diastolic volume (EDV) and end-systolic volume (ESV) were calculated from area and length measurements as $8(\mathrm{LVAd})^{2} / 3 . \mathrm{LVLd}$ and $8(\text { LVAs) })^{2} / 3 \pi$ LVLs, respectively. Ejection fraction was defined as 100 (EDV-ESV)/EDV. From the short-axis B-mode echocardiograms we determined the left ventricular wall thickness at two sites. In addition to wall thickness, LV internal chamber diameters were determined in diastole (LVIDd) and systole (LVIDs).

Left ventricular contractility was evaluated at the end of the study (137). For this purpose rats were anaesthetized with pentobarbital (50 mg.kg. ${ }^{-1}$ i.p., Sigma). A highfidelity catheter tip micromanometer (Mikro-tip 1.4F; SPR-671, Millar Instruments, Houston, TX, USA) was inserted into the left ventricular cavity through the right carotid artery. Ventricular pressure was measured and sampled at a rate of $2 \mathrm{kHz}$ under an I.V. ramp infusion of dobutamine up to $5 \mathrm{ng} . \mathrm{gBW}^{-1} \cdot \mathrm{min}^{-1}$ (Sigma) using a microinjection pump (model 200 Series, KD Scientific, Boston, MA, USA). Maximal positive $(+d p / d t / p)$ and negative (-dp/dt/p) pressure development corrected for arterial pressure were determined. At the end of the experiment hearts were excised, washed with isotonic saline and weighed. Hearts were further incubated for $24 \mathrm{~h}$ in formalin and embedded into paraffin for histochemical staining or snap frozen for further molecular analyses.

The paraffin embedded specimens were cut into $4 \mu \mathrm{m}$ thick sections for Sirius Red staining. Perivascular (138) and interstitial endomyocardial fibrosis (130) were assessed. All Sirius Red stained sections were carefully scanned with a light 
microscope (DFC 280, Leica, Tokyo, Japan) connected to a computer using the NIH image-analysis system for histomorphometry at a magnification of $\times 200$. Immunohistochemistry for laminin was performed to assess cardiomyocyte size (antilaminin L9393 Sigma, St. Louis, USA). Individual surface areas of at least 20 horizontally cut endocardial cardiomyocytes were calculated and the average values were used for analysis. Capillary staining was performed and quantified as described by Rakusan (139).

Table 2.1 Primer sequences

\begin{tabular}{|c|c|c|c|}
\hline Plindet & & Sexpluative 5 & $\begin{array}{l}\mathrm{T} \\
(\mathrm{c})\end{array}$ \\
\hline$A M \cdot R$ & dimm & 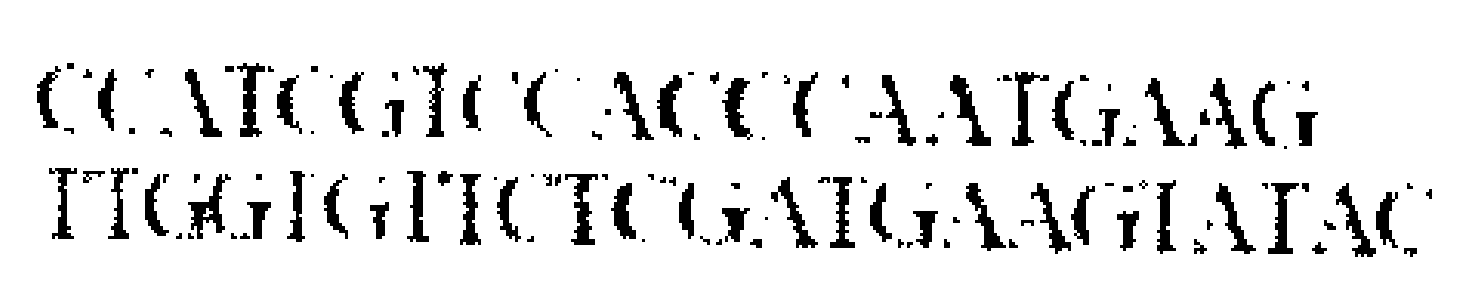 & 60 \\
\hline Exv & 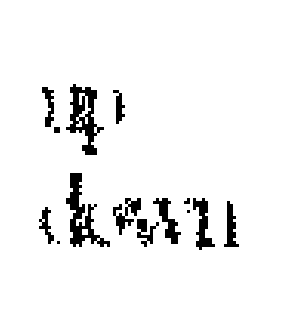 & 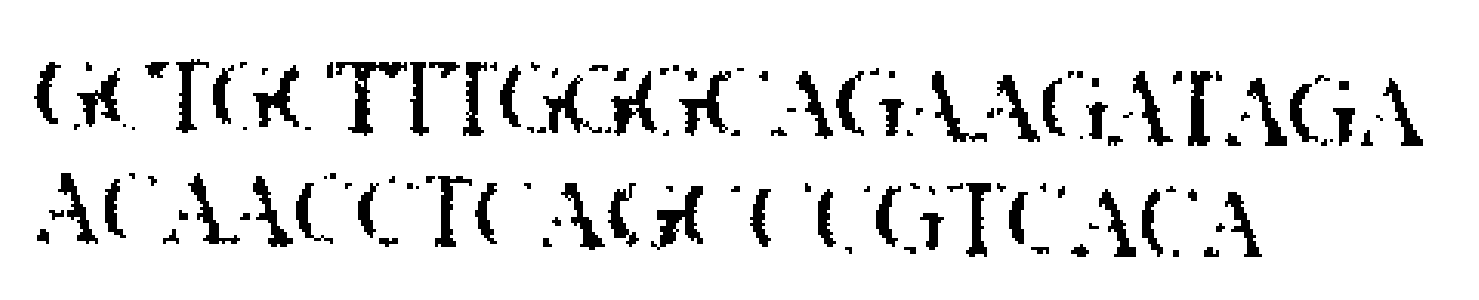 & 62 \\
\hline condugend & 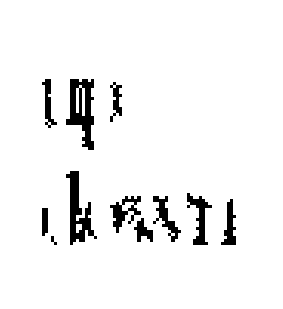 & 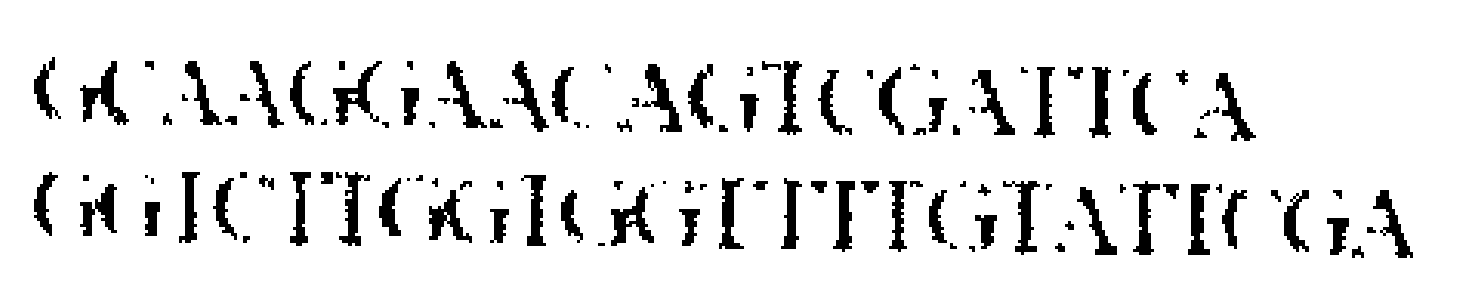 & 63 \\
\hline (MLustull & 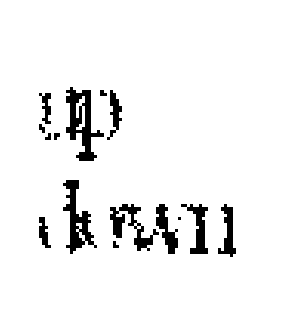 & 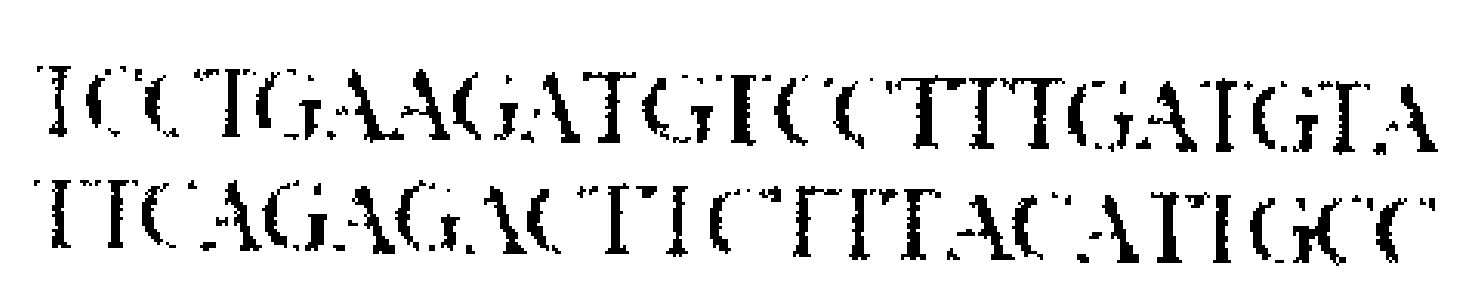 & 63 \\
\hline $5 x-1$ & 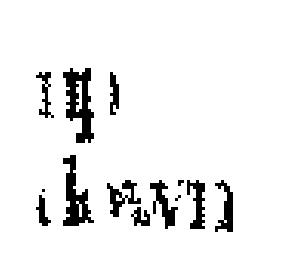 & 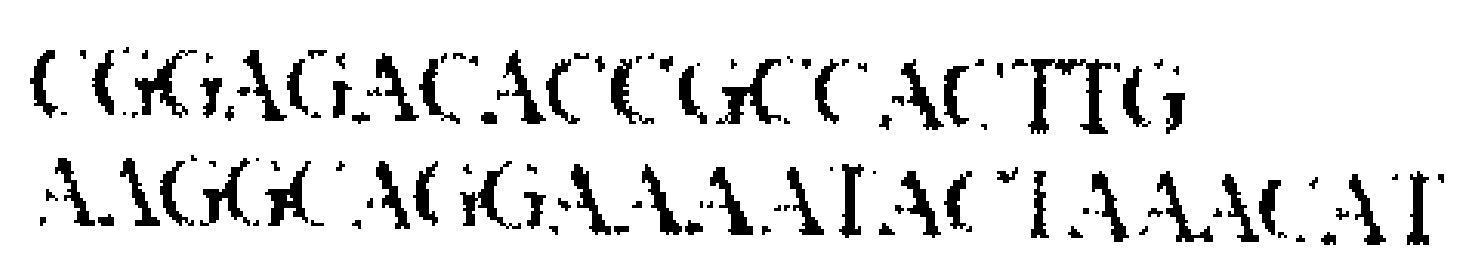 & 60 \\
\hline
\end{tabular}

RNA was isolated with an RNeasy Mini Kit from the snap frozen material (QIAGEN, Hilden, Germany). The RNA was further purified (Ultraspec-II RNA, Biotec Laboratories, Houston. Tx, USA) and quantified (Nanodrop ND-1000, WITEC, Switzerland). The RNA was transcribed into CDNA with Superscript III reversetranscriptase, using $250 \mathrm{ng}$ of random primers (Invitrogen Life Technologies).

Primers and probes against the selected genes of interest (collagen I and III and brain natriuretic peptide (140), angiotensin II type I receptor (96)) were designed from rat and human sequences available in GenBank using Primer Express Software (Table 2.1). Amplification and detection were performed using the ABI Prism 7700 Sequence 
Detection System and assessed relative to the expression level of a housekeeping gene, phosphoglucokinase-1 (PGK-1).

The 24-h urine excretion was collected in metabolic cages at the same time points as echocardiography was performed. Urinary albumin was measured immunonephelometric (BN-2 analyzer, Dade-Behring, Germany).

The kidneys were removed from animals under pentobarbital sodium anaesthesia, fixed in $4 \%$ formalin and embedded in paraffin. Central cross-sections of the whole kidney including cortex and medulla were prepared for examination. Glomerulosclerosis index (GSI) was measured in $4 \mu \mathrm{m}$ thick sections, stained with PAS. GSI was calculated by a nephropathologist in a blinded manner using a cumulative semiquantitative scoring as previously described by our group (141). GSI was calculated in both superficial and juxtamedullary glomeruli.

All parameters are expressed as means \pm S.E.M. Maximal changes in $+d p / d t / p$ and $\mathrm{dp} / \mathrm{dt} / \mathrm{p}$ evoked by dobutamine were compared by ANOVA and a post hoc Dunnett's Ttest. Parameters serially obtained by echocardiography were compared using an ANOVA for repeated measures and a post hoc Dunnett's T-test to identify the timerelated and between-group differences. $P$ values $\leq 0.05$ were regarded to indicate statistical significance.

\section{Results}

Transient prehypertensive treatment with losartan specifically attenuates hypertension and cardiac hypertrophy.

Brief antihypertensive treatment with hydralazine and losartan reduced significantly MAP. Hydralazine lowered blood pressure even stronger than losartan. Four weeks after hydralazine withdrawal blood pressure was restored to values observed in nontreated SHR whereas blood pressure remained lower in losartan treated SHR. HW/BW ratio was comparable in untreated and hydralazine treated SHR, while losartan treatment reduced this ratio significantly. Cardiac collagen deposition was lowest in SHR-Los at 12 weeks of age. Hydralazine treated SHR showed non-significantly lower collagen values as compared to untreated SHR (Table 2.2). 
Figure 2.1 Telemetry monitoring of blood pressure
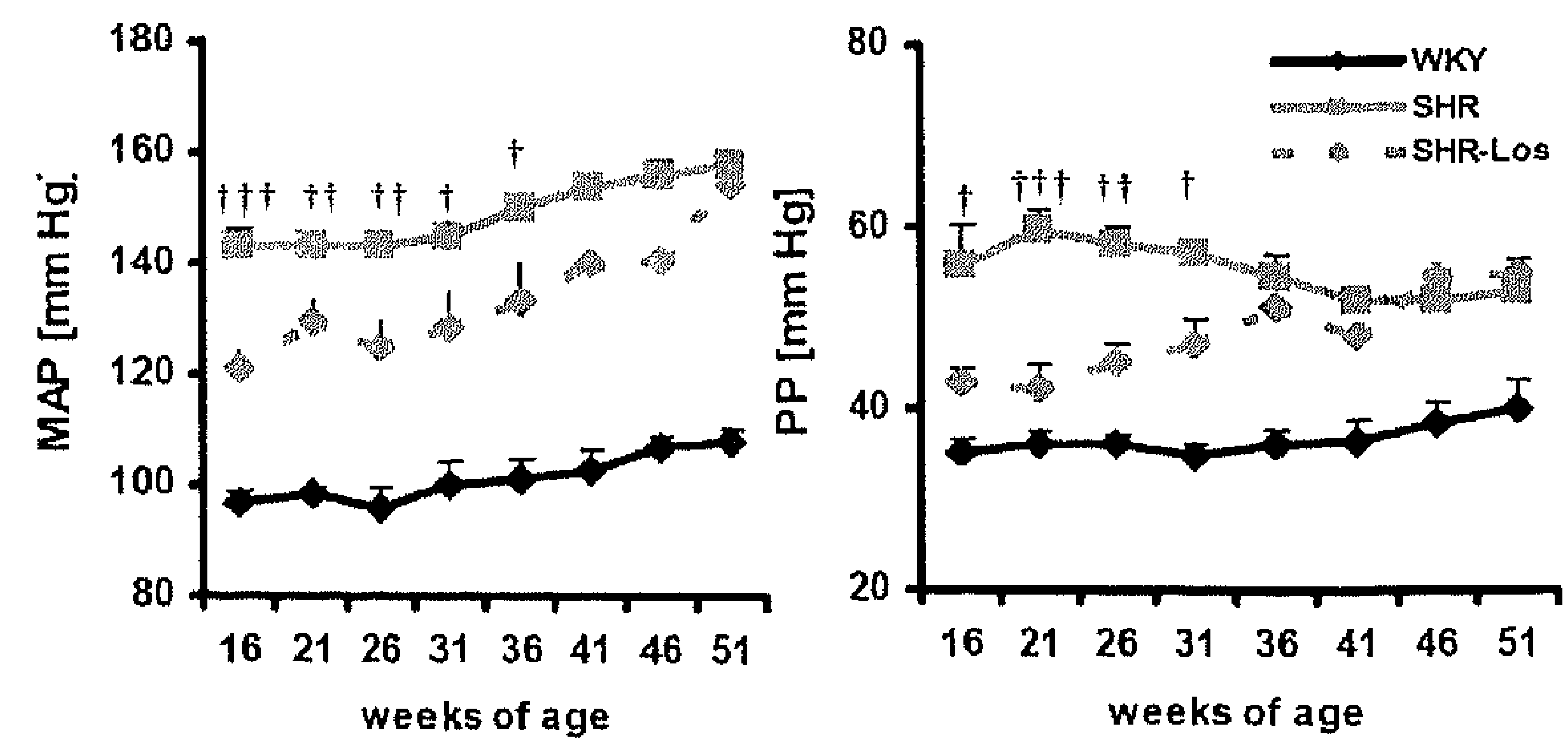

A) WKY mean arterial pressure (MAP) was significantly lower throughout the whole experiment $(P<0.001)$. MAP was significantly lower in SHR-Los compared to untreated SHR till week 36 , reflecting a period of 28 weeks after drug withdrawal.B) WKY pulse pressure (PP) was significantly lower throughout the whole experiment $(P<0.001)$. PP was significantly lower In SHR-Los compared to untreated SHR till week 31, reflecting a period of 23 weeks after drug withdrawal.

\section{Longitudinal blood pressure evaluation till week 55}

Mean blood pressure (MAP) and pulse pressure (PP) of the 3 groups are displayed in Figure 2.1. There was a prolonged blood pressure lowering effect in SHR-Los up to week 36 with a maximum decrease of MAP by $20 \mathrm{~mm} \mathrm{Hg}$. Thereafter a gradual increase in MAP towards untreated SHR level was noticed which was reached at week 50. Pulse pressure (PP) was decreased by transient prehypertensive losartan treatment up to $18 \mathrm{~mm} \mathrm{Hg}$. The gradual increase in PP started at week 28, but remained significant till week 32 . At 72 weeks of age systolic blood pressure values of WKY were $129 \pm 6 \mathrm{mmHg}$. Blood pressure values did not differ significantly (SHR $221 \pm 11$, SHR-Los $204 \pm 16 \mathrm{mmHg}$ ) in untreated SHR and SHR-Los. 
Figure 2.2 Longitudinal echocardiography

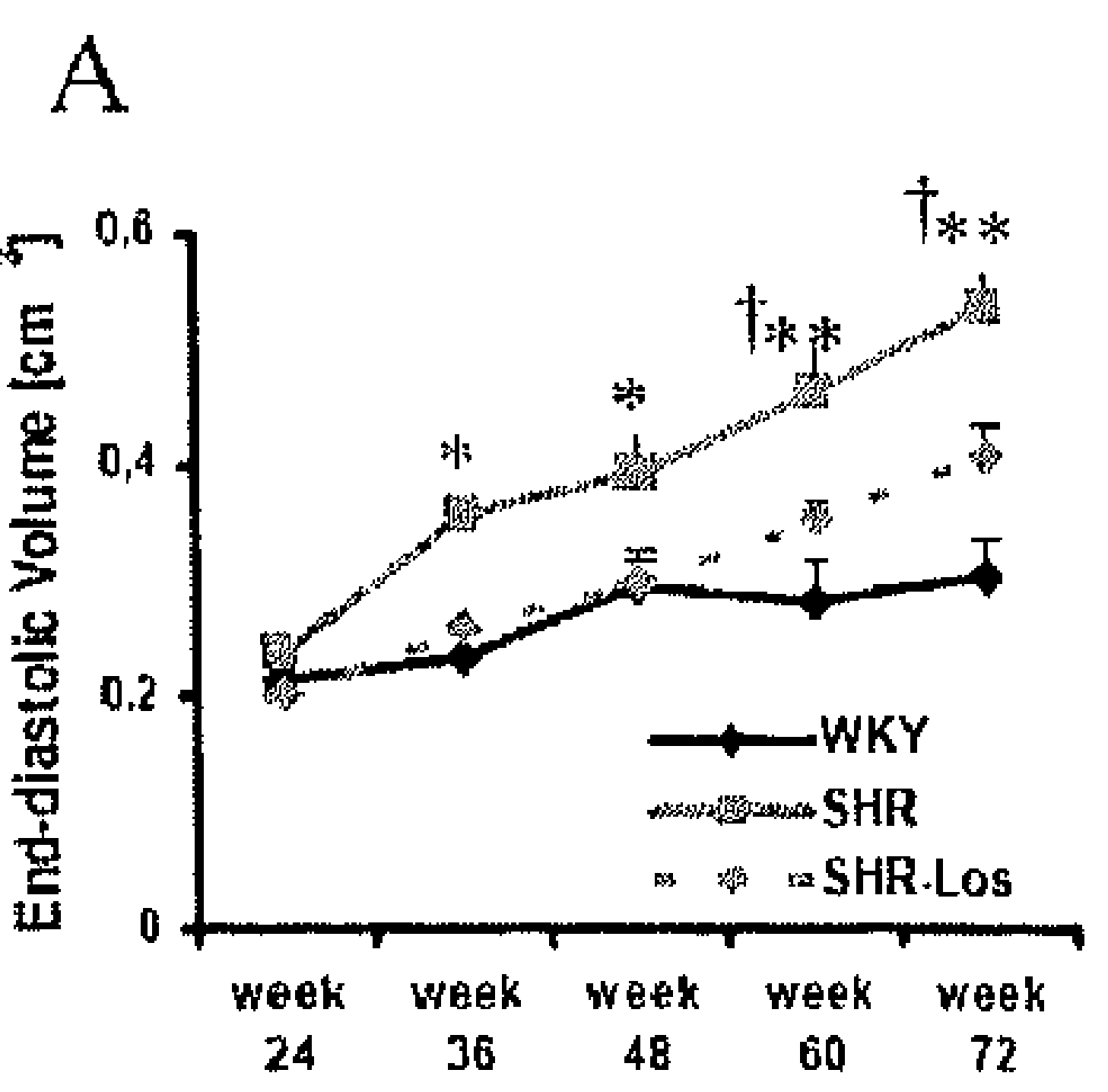

$\mathrm{B}$

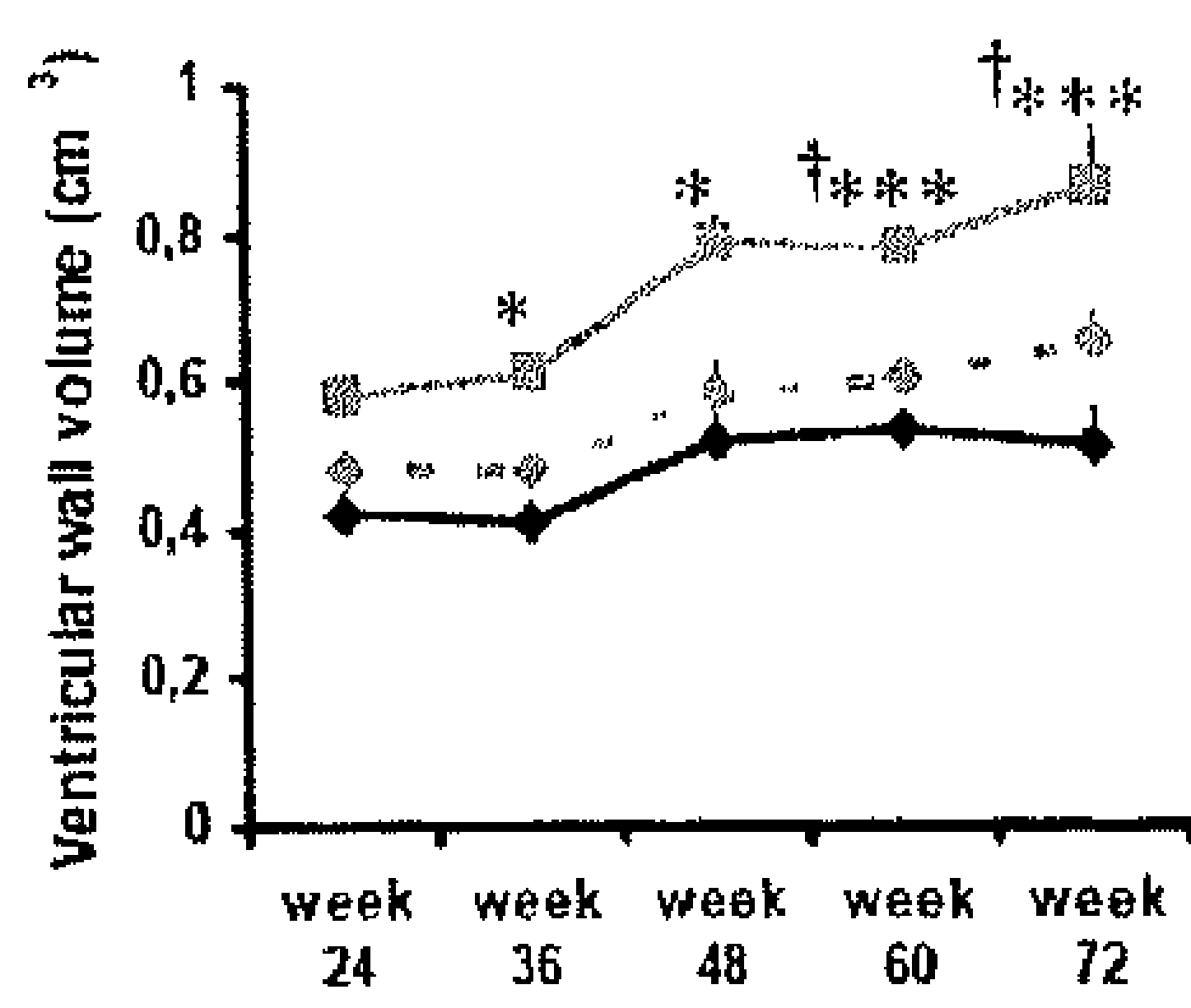

C

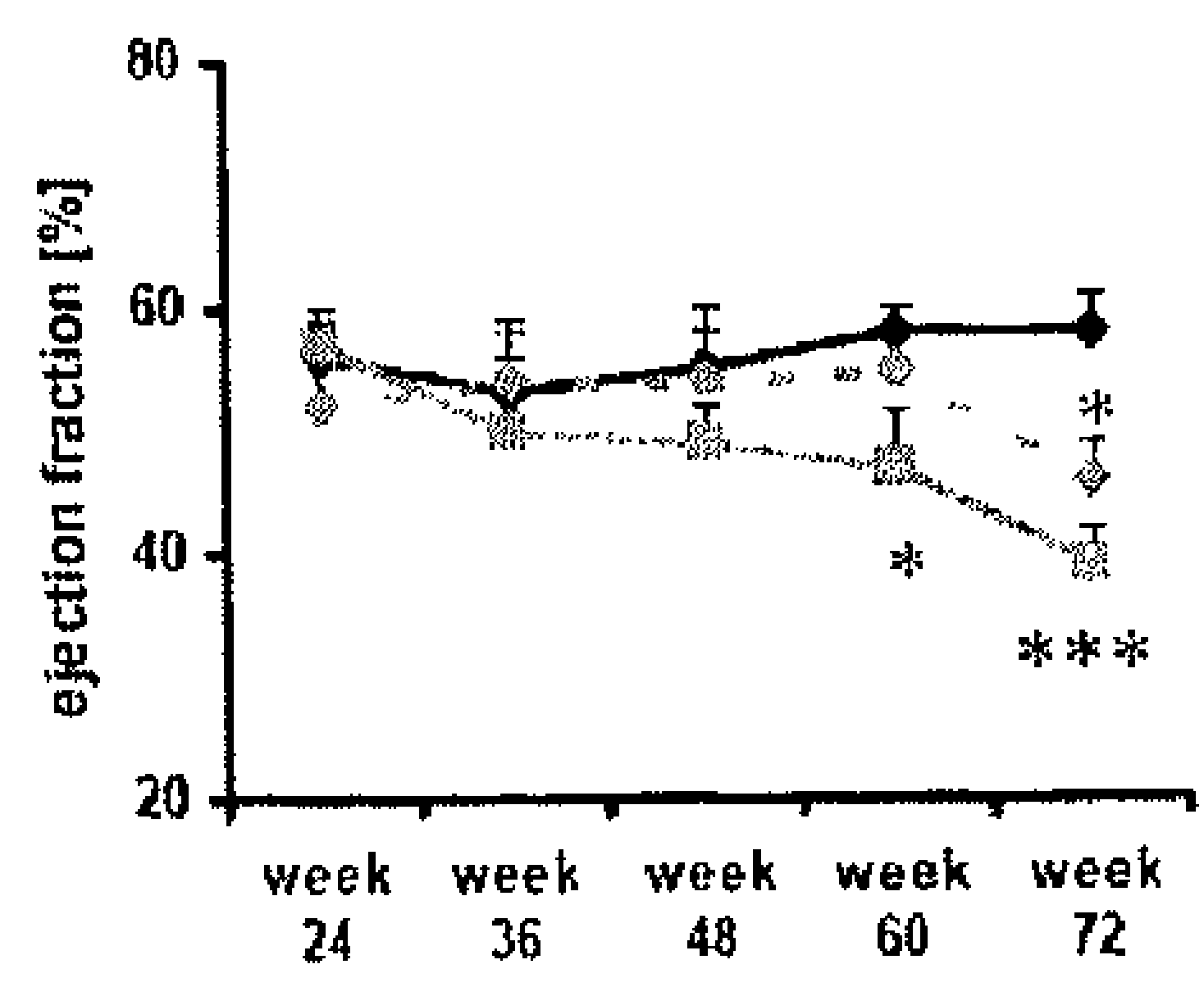

$* P<0.05, * * P<0.01, * * * P<0.001$ vs. WKY: $+P<0.05$ vs. SHR-Los

A) End-diastolic volume (EDV) started to increase significantly in untreated SHR at week 36 $(P<0.05)$. This process further developed throughout the experiment compared to WKY $(P<0.01)$. In contrast, EDV of SHR-Los did not increase significantly compared to WKY till advanced age. B) Ventricular wall volume (VW) started to increase significantly in untreated SHR at week 36 $(P<0.05)$. This process further developed throughout the experiment compared to WKY $(P<0.001)$. In contrast, VW of SHR-Los did not increase significantly compared to WKY till advanced age. C) Ejection fraction (EF) started to decline significantly in untreated SHR at week $60(P<0.05)$. This process further developed till week $72(P<0.001)$. SHR-Los revealed comparable EF till week 60. EF was significantly decreased compared to WKY at week $72(P<0.01)$

\section{Longitudinal evaluation of cardiac function till week 72}

Longitudinal assessments of structural and functional cardiac properties are summarized in Figure 2.2A-C. The EDV was comparable in all three groups at week 24 (range: $0.20-0.24 \mathrm{~cm}^{3}$ ). Thereafter untreated SHR demonstrated a stronger increase in EDV compared to WKY. This difference became significant at week 36 (SHR $0.36 \pm 0.02 \mathrm{~cm}^{3}$ versus WKY $0.24 \pm 0.03 \mathrm{~cm}^{3}, P<0.05$ ) and further augmented till week 72 (SHR $0.54 \pm 0.04 \mathrm{~cm}^{3}$ versus WKY $0.31 \pm 0.04 \mathrm{~cm}^{3}, P<0.01$ ). In contrast, EDV in SHR-Los showed similar values as in WKY till week 36 (SHR-Los $0.26 \pm 0.01 \mathrm{~cm}^{3}$ ). A small non-significant incline was noted over time in SHR-Los (week 72: $\left.0.41 \pm 0.03 \mathrm{~cm}^{3}\right)$. At 60 and 72 weeks of age EDV of SHR-Los was significantly smaller than in untreated SHR $(P<0.05)$.

\section{Longitudinal evaluation of cardiac function till week 72}

Longitudinal assessments of structural and functional cardiac properties are summarized in Figure 2.2A-C. The EDV was comparable in all three groups at week 24 (range: $0.20-0.24 \mathrm{~cm}^{3}$ ). Thereafter untreated SHR demonstrated a stronger 
increase in EDV compared to WKY. This difference became significant at week 36 (SHR $0.36 \pm 0.02 \mathrm{~cm}^{3}$ versus WKY $0.24 \pm 0.03 \mathrm{~cm}^{3}, P<0.05$ ) and further augmented till week 72 (SHR $0.54 \pm 0.04 \mathrm{~cm}^{3}$ versus WKY $0.31 \pm 0.04 \mathrm{~cm}^{3}, P<0.01$ ). In contrast, EDV in SHR-Los showed similar values as in WKY till week 36 (SHR-Los $0.26 \pm 0.01 \mathrm{~cm}^{3}$ ). A small non-significant incline was noted over time in SHR-Los (week 72: $\left.0.41 \pm 0.03 \mathrm{~cm}^{3}\right)$.At 60 and 72 weeks of age EDV of SHR-Los was significantly smaller than in untreated SHR $(P<0.05)$.

At week 24 the LV mass $(\mathrm{VW})$ of SHR $\left(0.58 \pm 0.03 \mathrm{~cm}^{3}\right)$ was non-significantly greater than in WKY $\left(0.41 \pm 0.02 \mathrm{~cm}^{3}\right)$ or SHR-Los $\left(0.48 \pm 0.03 \mathrm{~cm}^{3}\right)$. However, from 36 weeks on, differences in LV mass between untreated SHR and WKY were statistically significant and maximal at week 72 (SHR $0.87 \pm 0.08 \mathrm{~cm}^{3}$ versus WKY $0.51 \pm 0.05 \mathrm{~cm}^{3}$, $P<0.001)$. Throughout the study period LV mass was non-significantly greater in SHRLos than in WKY. LV mass of SHR-Los was lower than in untreated SHR (SHR $0.78 \pm 0.04 \mathrm{~cm}^{3}$ versus SHR-Los $0.61 \pm 0.02 \mathrm{~cm}^{3}, P<0.05$ ) at week 60 and 72 .

Table 2.3 Left ventricular function at 72 weeks of age

\begin{tabular}{cccc}
\hline & DPP max $\left(\mathrm{s}^{-1}\right)$ & DPN max $\left(\mathrm{s}^{-1}\right)$ & BNP mRNA vs. PGK-1 \\
\hline WKY & $220 \pm 8$ & $114 \pm 10$ & $1 \pm 0.1$ \\
SHR & $114 \pm 11^{* * * *}$ & $71 \pm 6^{* * *}$ & $2.4 \pm 0.1^{* * * *+\dagger}+\dagger$ \\
SHR-Los & $187 \pm 17$ & $87 \pm 8$ & $1.5 \pm 0.1^{*}$ \\
\hline
\end{tabular}

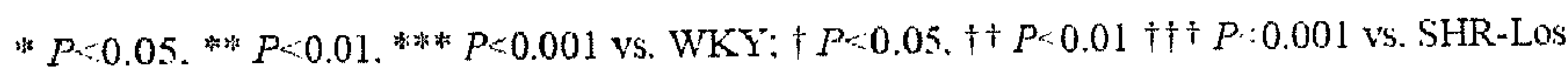

Left ventricular EF was comparable in all three groups till week 48 . The EF deteriorated significantly in untreated SHR as compared to WKY (SHR $47 \pm 4 \%$ versus WKY $58 \pm 2 \%, P<0.05$ ) at week 60 . This process further augmented till week 72 (SHR $39 \pm 3 \%$ versus WKY $58 \pm 3 \%, P<0.01)$. SHR-Los displayed comparable EF values as WKY till week 60 (SHR-Los $55 \pm 5 \%$ ). A significant decline in EF was observed in SHRLos as compared to WKY $(46 \pm 3 \%, P<0.05)$ at week 72 . 


\section{Cardiac state at advanced age (week 72 of age)}

Table 2.3 presents LV function evaluated as the contraction rate $(+\mathrm{dp} / \mathrm{dt} / \mathrm{p})$ and relaxation rate $(-d p / d t / p)$ in 72 weeks old rats. $+d p / d t / p$ and $-d p / d t / p$ were significantly higher in WKY than in untreated SHR $(P<0.01)$. Values of $+d p / d t / p$ in SHR-Los were situated between WKY and untreated SHR values without reaching significant differences. $-d p / d t / p$ of SHR-Los was significantly reduced as compared to WKY $(P<0.05)$. BNP mRNA as indicator of heart failure was significantly higher in untreated SHR than in WKY $(P<0.001)$ and SHR-Los $(P<0.001)$. SHR-Los BNP mRNA remained elevated when compared to levels found in WKY $(P<0.05)$.

HW/BW ratios and morphological data concerning collagen deposition, myocyte size and arteriolar properties are displayed in Table 2.4.. HW/BW ratio was significantly higher in untreated SHR than in WKY $(P<0.001)$. Prehypertensive treatment partially prevented cardiac hypertrophy as compared to untreated SHR $(P<0.01)$.

Table 2.4 Characteristics of cardiac hypertrophy at 72 weeks of age

\begin{tabular}{|c|c|c|c|c|c|c|}
\hline & $\begin{array}{l}\text { HW } \\
\text { (gikg) }\end{array}$ & $\begin{array}{c}\text { Intarstitial } \\
\text { collagen }(\theta)\end{array}$ & $\begin{array}{c}\text { Perivascular } \\
\text { collagen humen } \\
\text { area }\end{array}$ & $\begin{array}{c}\text { Collagen III } \\
\text { aRNA vs PGK- } \\
1\end{array}$ & $\begin{array}{c}\text { Afyocyte size } \\
\text { (cmi) }\end{array}$ & $\begin{array}{c}\text { Capillaty density } \\
\left(\operatorname{minn}^{3}\right)\end{array}$ \\
\hline WKY & $3.6=0.2$ & $1.8=0.3$ & $1.2 \pm 0.2$ & $1.0=0.1$ & $286 \div 11$ & $3550=\$ 5$ \\
\hline SHR & $5.2=0.2 * * * 8$ & $\begin{array}{c}6.5 \pm 1.3 * * * \\
\dagger\end{array}$ & $3.9=0.4 * 8 * \dagger$ & $2.0=0.1 * * * \div+\dagger$ & $408=8 * * * \dagger+t$ & $2180 \pm 1403 * * * \dagger$ \\
\hline SHR-LOS & $4.7=0.1 * *$ & $2.4=0.3$ & $2.4 \pm 0.4$ & $1.0 \pm 0.1$ & $334 \pm 6^{* * *}$ & $3260=220$ \\
\hline
\end{tabular}

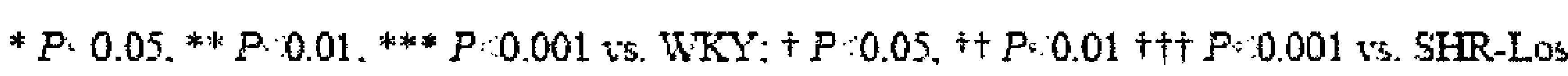

Cardiomyocytes were significantly hypertrophied in untreated SHR compared to WKY $\left(286 \pm 11 \mu \mathrm{m}^{2}\right.$ versus $\left.408 \pm 8 \mu \mathrm{m}^{2}, P<0.001\right)$. Intermediate values for cardiomyocyte size were found in prehypertensive losartan treated SHR $\left(334 \pm 6 \mu \mathrm{m}^{2}\right)$. Capillary density was lowest in untreated SHR $(P<0.001)$, whereas in SHR-Los values close to those observed in normotensive WKY were found. Left ventricular interstitial collagen deposition was fully attenuated in SHR-Los whereas perivascular fibrosis was only partially prevented $(P<0.05)$. Type I collagen mRNA did not show any differences 
between the groups (data not shown). In contrast, type III collagen mRNA was 2-fold upregulated in untreated SHR only.

\section{Cardiac angiotensin signaling at advanced age}

AT1R mRNA was 2-fold higher in old untreated SHR than in WKY and SHR-Los $(P<0.001)$. Data are depicted in Figure 2.3.

Figure 2.3 Semiquantitative analysis of gene expression levels of the AT1R

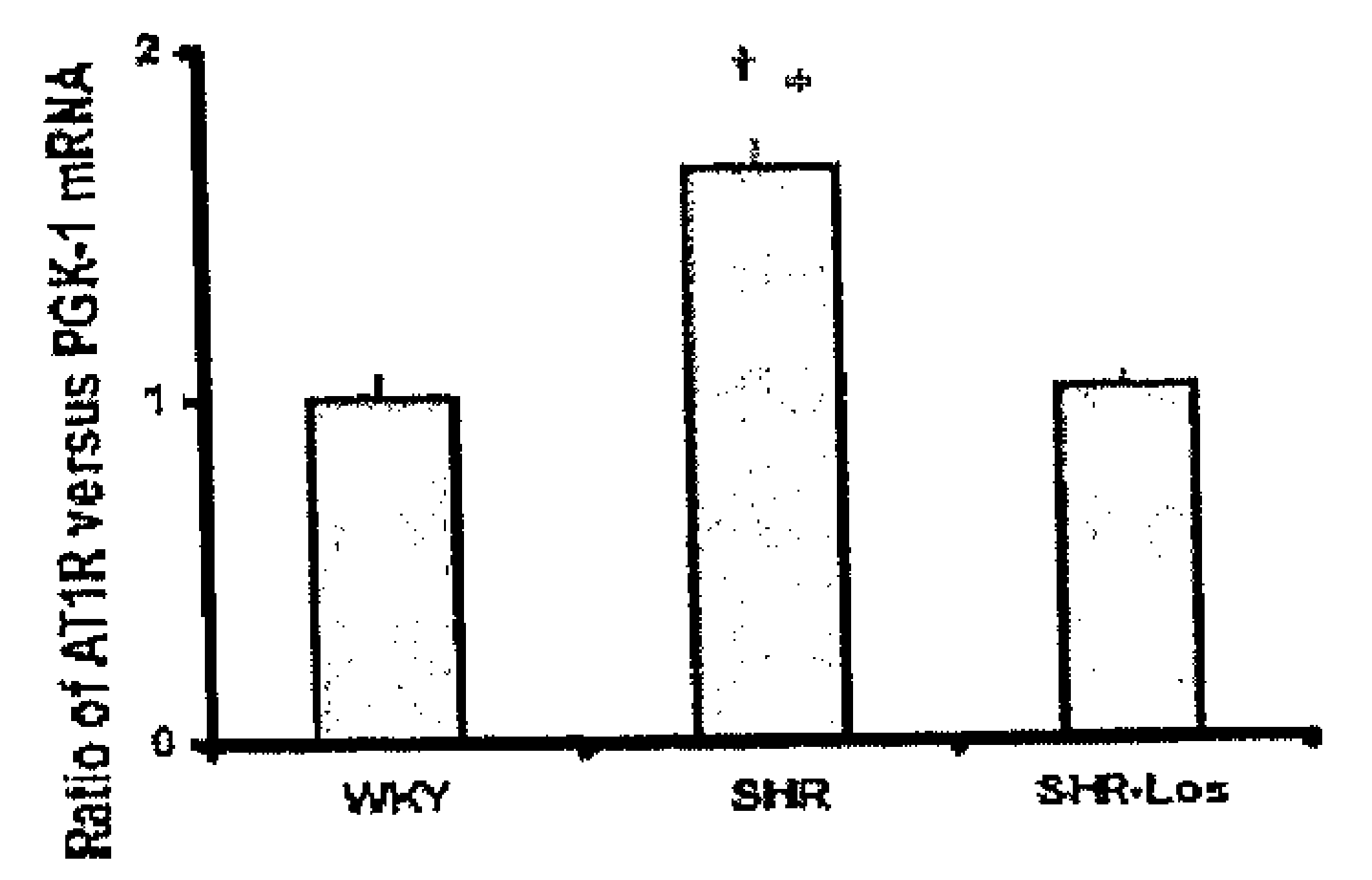

Gene expression of angiotensin If type 1 receptor (AT1R) mRNA was significantly increased in untreated SHR compared to WKY $\left(^{*}\right)$ and SHR-Los $(\dagger)$, respectively $(P<0.001)$

\section{Renal state until advanced age}

Compared to WKY, 24-h albuminuria was increased in untreated SHR throughout the study period. A steep increase in albumin excretion was noticed in untreated SHR $(12.0 \pm 1.3$ $\mathrm{mg} / \mathrm{day}, P<0.001$ ) at 60 weeks of age. This surge was blunted in pre-hypertensively losartan treated SHR $(8.9 \pm 0.8 \mathrm{mg} /$ day, $P<0.01)$. However, albuminuria remained significantly higher in these rats than in WKY $(3.1 \pm 0.7 \mathrm{mg} /$ day). At 72 weeks of age $24-\mathrm{h}$ albuminuria remained low in WKY $(9.6 \pm 0.8 \mathrm{mg} /$ day), but increased in SHR $(80.3 \pm 21.5 \mathrm{mg} /$ day, $P<0.001)$ and SHRLos (40.8 $\pm 4.6 \mathrm{mg} / \mathrm{day}, P<0.01)$. Glomerular morphology at 72 weeks of age showed enhanced sclerosis in untreated SHR as compared to WKY. This pattern was most pronounced in juxtamedullary glomeruli. Intermediate values were found in prehypertensively losartan treated SHR (Figure 2.4). 
Figure 2.4 Glomerular function and structure

A

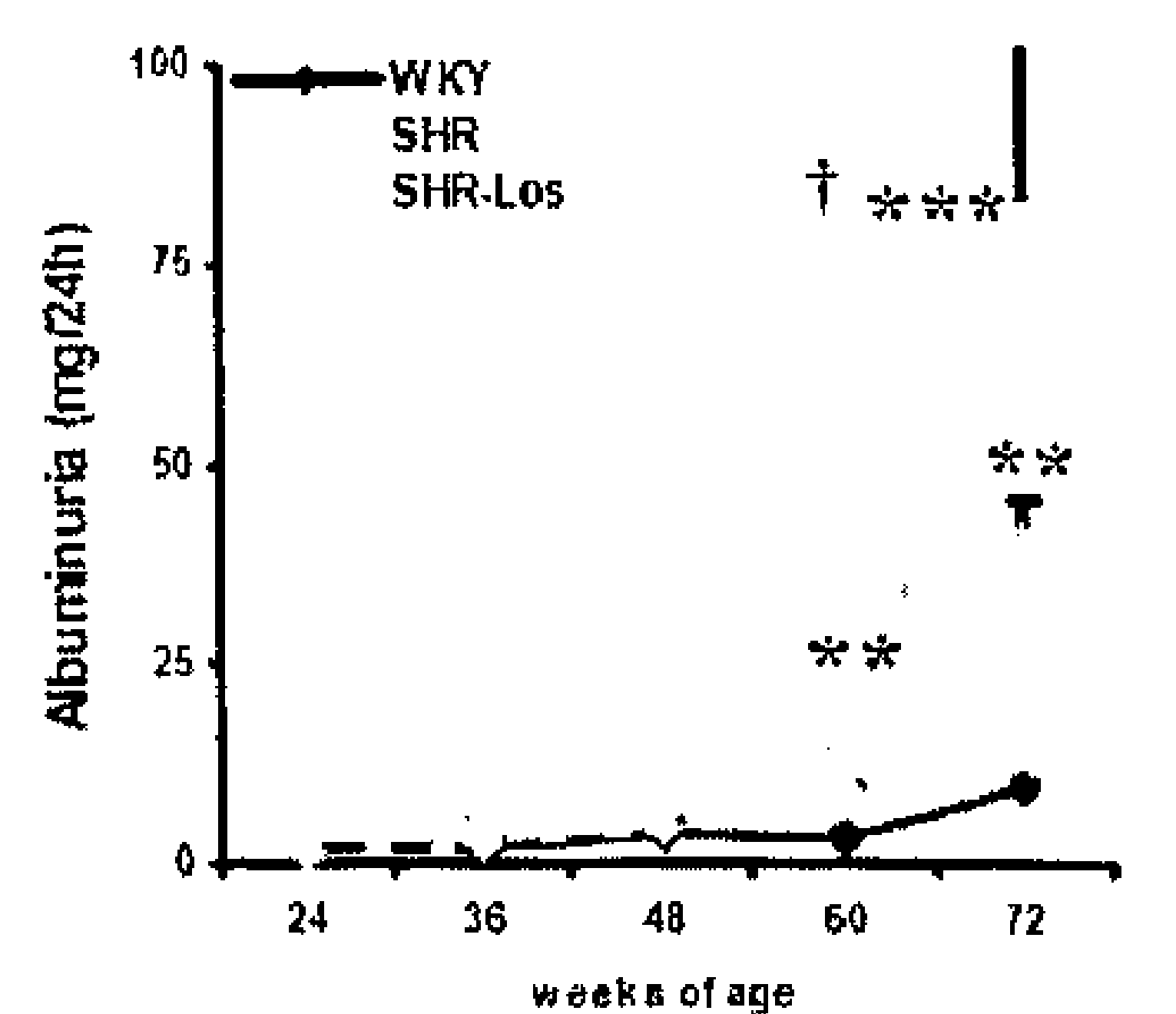

$\mathrm{B}$

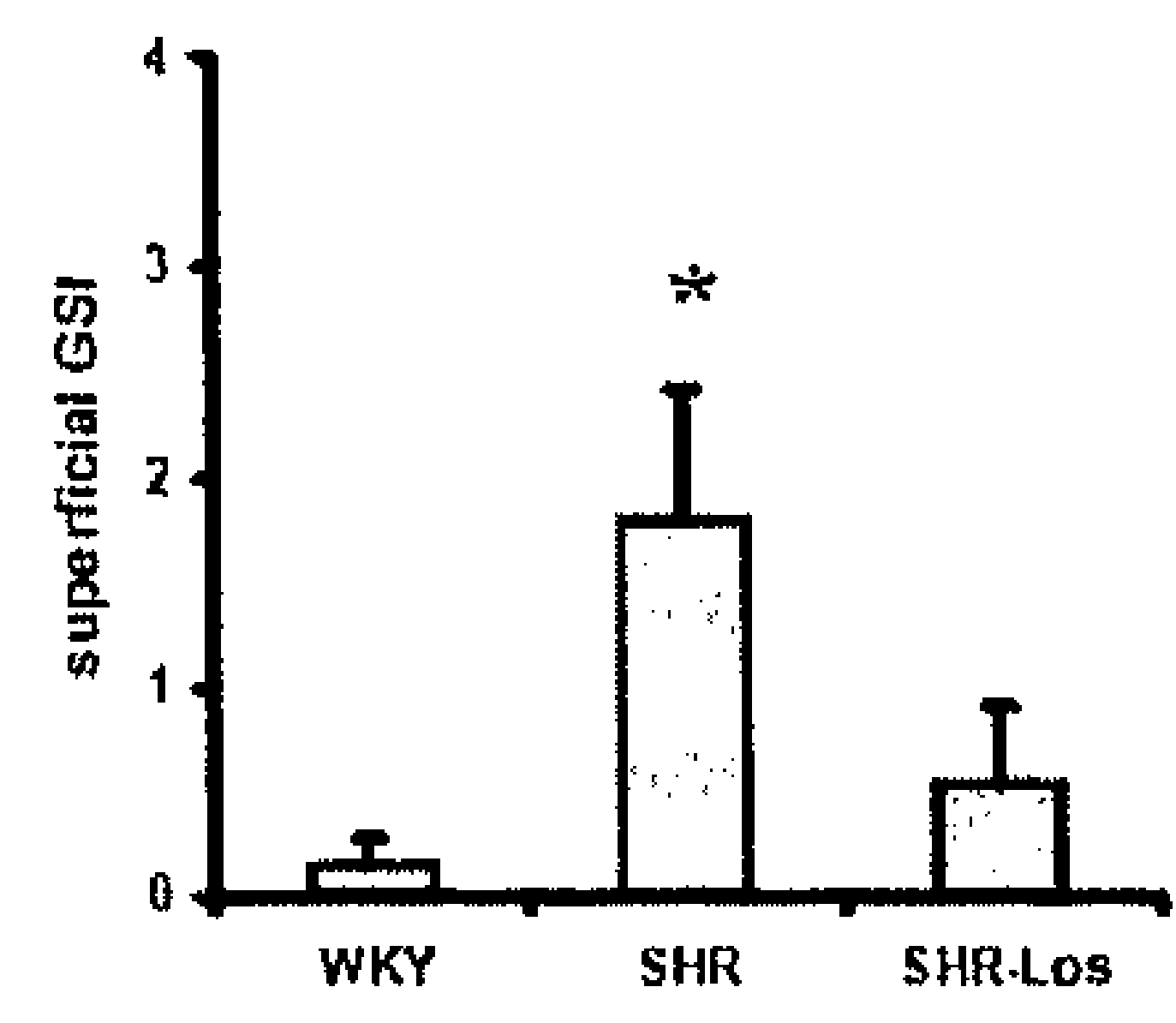

C

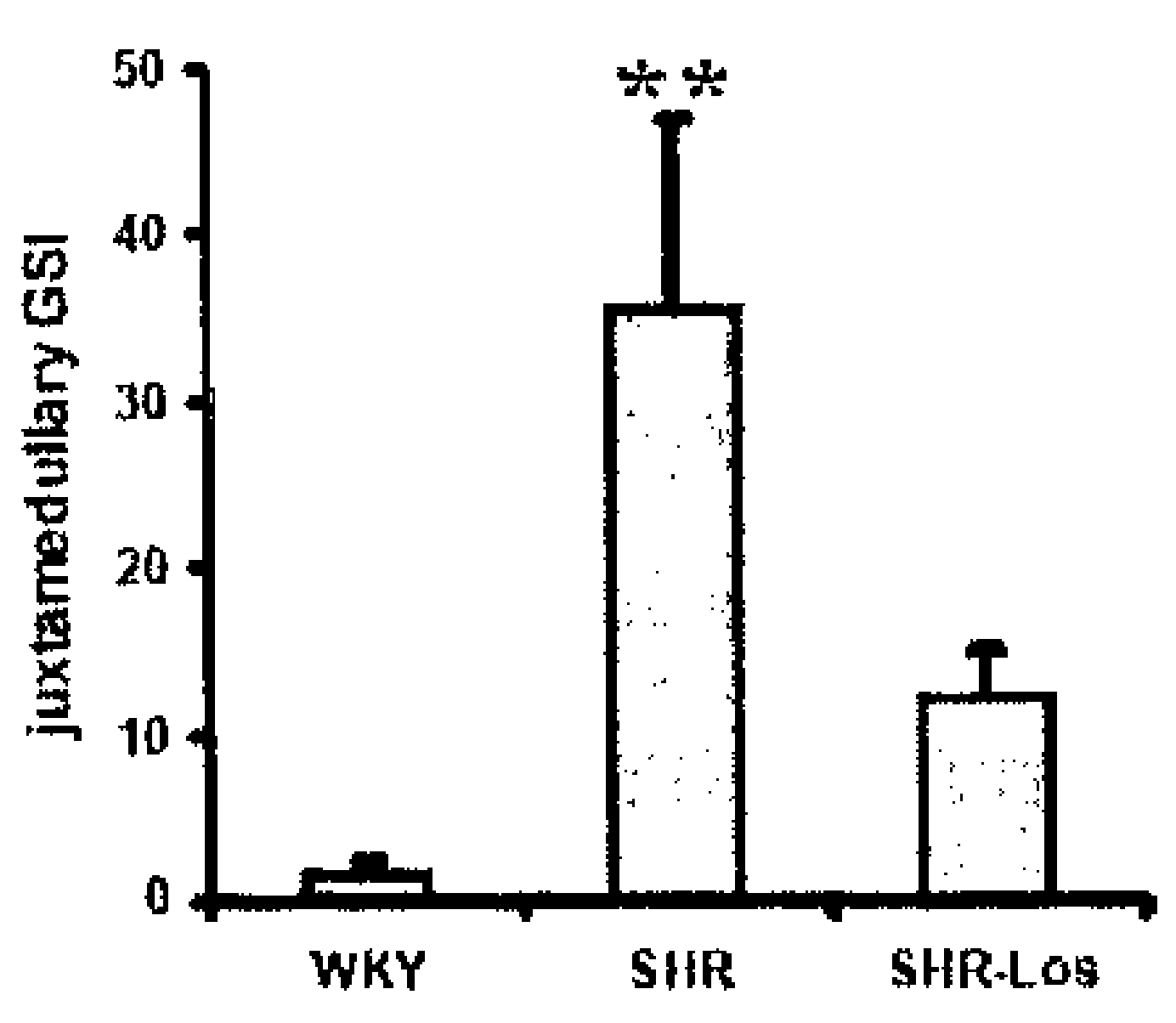

A) 24-h albuminuria was determined in 3-monthly intervals. Albuminuria was significantly increased at 60 weeks of age in both SHR groups $\left({ }^{* *} P<0.01\right)$. This process continued at 72 weeks of age in untreated SHR ( $\left.{ }^{\star \star \star} P<0.001\right)$ and to significantly lesser extent in SHR-Los ( ${ }^{\star \star} P<0.01$ vs. WKY and $+P<0.05$ vs. SHR). B) Superficial glomerufi of untreated SHR show slightly elevated sclerosis as compared to WKY $\left({ }^{*} P<0.05\right)$. C) Juxtamedullary glomeruli show pronounced sclerosis in untreated SHR as compared to WKY $\left(^{* *} P<0.01\right)$. SHR-Los show less sclerosis.

\section{Discussion}

Transient prehypertensive angiotensin II type 1 receptor antagonism, and not early blood pressure lowering, leads to sustained cardiac protection until advanced age in untreated SHR. Our data show a prolonged cardioprotective effect on a functional, morphological and molecular level up to 72 weeks of age. This is of particular interest as cardiac protection sustained in transiently treated SHR despite the fact that blood pressure lowering was only apparent up to 36 weeks of age.

The first set of experiments was performed to investigate whether the initial blood pressure lowering or rather effects caused by AT1R blockade are responsible for the prolonged blood pressure and target organ effects. The data demonstrate that prehypertensive treatment with the general vasodilator hydralazine did neither lower blood pressure for a prolonged period nor reduced cardiac hypertrophy. In contrast, AT1R antagonism had prolonged effects on both, suggesting that direct effects of AT1R blockade on the heart underlie the long-term protective effect, which confirms earlier observations by Bergstrom (104). Prehypertensive treatment with AT1R blockers does not affect the heart only. Also the kidney is remodeled. Because cardiac damage in the untreated SHR is preceded by renal damage $(142,143)$ it is therefore likely that additional renal protective effects may have 
been responsible for the present cardiac protective effects of losartan too. The study wa not designed to test this hypothesis and clearly more work is needed in this area.

Transient prehypertensive treatment with losartan significantly attenuated the bloo pressure development in SHR up to 36 weeks of age. The maximal blood pressure lowerin effect that was reached by this treatment regimen was about $20 \mathrm{mmHg}$ and was mos pronounced at 16 weeks of age. From then on blood pressure rose gradually to level observed in untreated SHR within 36 weeks. These data extend similar observations the were achieved in transiently losartan treated SHR by Bergstrom et al. up to 24 weeks of ag and are in support of theories that stress the crucial role of AT1 receptor activation in lons term setting of blood pressure (104). The new finding of the present study is th: prehypertensive treatment did not lead to life-long blood pressure lowering as a restoratio of hypertensive blood pressure levels took place during adulthood. The second main findin is that cardiac protection was maintained until advanced age even after restoration of bloo pressure. Comparable observations on cardiac remodeling by early AT1-receptor blockad were made before in SHR, but only on the basis of HW/BW ratios. However, these studie were considerably less extended with regards to observation time and limited to the peric in which blood pressure lowering was apparent $(103,104,111)$.

The longitudinal echocardiographic approach allowed us to investigate the progression । cardiac dilatation and hypertrophy on a structural (EDV and LV mass) and functional (EI level in a non-invasive manner (143). In combination with the blood pressure data we ce give further insight in the blood pressure related development of cardiac damage.

EDV and LV mass were nearly identical in WKY and transiently treated SHR during th period of blood pressure lowering, while untreated SHR showed a significant progression EDV and LV mass. Thus, the accumulation of damage was reduced during the period prolonged blood pressure reduction. This led to a significantly reduced LV dilatation ar hypertrophy at 36 weeks of age. After hypertensive blood pressure values were restored SHR-Los, the rate of increase in EDV and LV mass occurred in parallel to the chang observed in untreated SHR. This suggests that blood pressure is an important determina of LV dilatation and hypertrophy, because from week 36 on, the rate of change in cardi: dilatation and hypertrophy was similar in SHR-Los and untreated SHR. Prehypertensi 
treatment with losartan seems to postpone the cardiac remodeling process. The assumption that the blood pressure lowering is of major relevance for the cardioprotection has been suggested before $(103,104,111)$. The new finding of this study is that cardioprotective effects of transient treatment with losartan remain visible until 72 weeks of age and are not caught up. This is of importance as it postpones the further development of cardiac hypertrophy and dilatation when antihypertensive therapy is not effective anymore. As a consequence critical ranges of cardiac structure are reached at a later stage which decreases the risk for cardiovascular complications. In context with the advanced age of the rats it is of importance to remember that a postponed cardiovascular complication is comparable to an erased complication.

In contrast to the effects regarding EDV and LV mass, cardiac function (EF) deteriorates at 60 weeks of age in SHR irrespective of treatment. This cannot be explained by the increased cardiac dilatation or hypertrophy alone. Interestingly, albuminuria markedly increased in both SHR groups at the same time as EF dropped. Albuminuria is known to increase when glomerular function deteriorates which occurs in SHR at 60 weeks of age (142). Reduced renal function is known to affect cardiac function and increases markers for heart failure $(142,144)$. Thus, the drop of $E F$ in both SHR groups can be understood as cardio-renal feedback, which was not primarily linked with EDV and LV mass. The role of the cardio-renal feedback is further strengthened by the fact that prehypertensively losartan treated SHR showed less albuminuria and glomerulosclerosis and as a consequence a delayed and less pronounced drop of EF and left ventricular contractility as found in untreated SHR $(140,143)$.

The diastolic function remained improved in SHR-Los as compared to untreated SHR until 72 weeks of age. This was corroborated by the following histological and molecular features: perivascular and interstitial fibrosis as well as LV collagen III mRNA content were markedly lower in SHR-Los than in untreated SHR with values close to those found in normotensive WKY. Other important characteristics of cardiac hypertrophy, such as myocyte size and capillary density were also favorably altered in SHR-Los (145-148). The fact that several factors involved in diastolic function did not differ between WKY and prehypertensively treated SHR-Los rose the question whether beside a "non-catch-up" and a reduced renal damage other factors are involved in the maintained cardioprotection. 
In this context it is important to realize that the majority of successful prehypertensive interventions are based on temporary blockade of the RAS. Our data furthermore demonstrates that prehypertensive therapy with equipotent blood pressure lowering but without RAS effects has neither prolonged blood pressure lowering nor target organ protecting effects. This suggests that the prehypertensive RAS blockade plays a central role in the long-term cardioprotection. This role is further strengthened by data showing increased AT1R density in cardiac hypertrophy $(126,149,150)$ and that a low AT1R blockade enables reversal of this process (107). Therefore, the AT1R is a potential candidate for such a protective factor. And indeed, we demonstrated that these rats show a markedly reduced cardiac AT1R gene expression as compared to the untreated SHR even at advanced age. Reduced ATIR gene expression might play a potential protective role beside blood pressure or cardio-renal feedback.

Irrespective of the underlying mechanisms, our data have important implications for the clinical situation (121). Our findings show that a simple, inexpensive and short-duration pharmacological intervention, can achieve long-lasting cardiovascular protection. This is of particular relevance, given the fact that hypertension related cardiac remodeling and cardiac function impairment affect a substantial part of the hypertensive population and poses a huge burden on health and health care related costs. As such, the idea to be able to provide long lasting protection against hypertensive cardiomyopathy using a preventive treatment with well established low cost drugs is particularly appealing (121). Although other options in that direction as gene therapy exist (151), the advantage of the drug approach is that based on technical, economic and ethical issues, feasibility seems more within reach. Therefore, clinical trials testing this approach in a human setting would be highly desirable.

In summary, we have shown that transient prehypertensive angiotensin II receptor blockade, but not early blood pressure reduction, leads to sustained cardiac protection till advanced age, offering a potential therapeutic benefit for humans by lowering cardiovascular morbidity. 
CHAPTER THREE:

Prehypertensive RAAS blockade in SHR ameliorates long-term vascular function

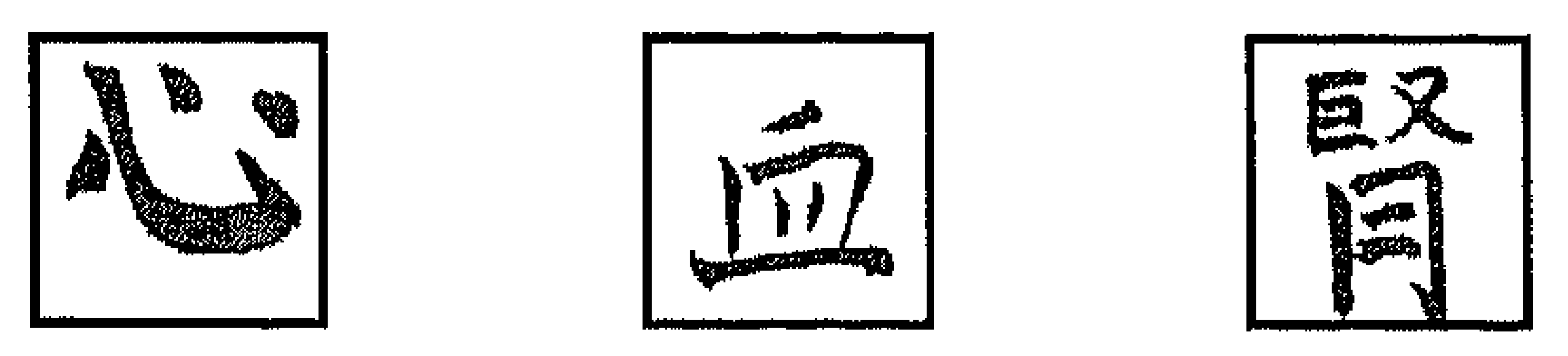

Marcus Baumann, Remco Megens, Roger Bartholome, Sebastian Dolff, Marc A.J.M. van Zandvoort, Jos F.M. Smits, Harry A.J. Struijker Boudier, Jo G.R. De Mey

Hypertension Res 2007: 30(9): 853-861. 


\begin{abstract}
Background: Arterial function after long-term hypertension is characterized by remodeling, endothelial dysfunction and reduction of previously enhanced contractile responses. We investigated whether transient prehypertensive RAAS blockade modifies long-term arterial function.

Methods: WKY (i) and SHR (ii) were prehypertensively (week 4-8) treated with losartan (iii) or spironolactone (iv) $(20,0.5 \mathrm{mg} / \mathrm{kg} /$ day, respectively) and studied at 8 and 72 weeks of age. Systolic blood pressure (SBP) was measured intra-arterially. In isolated mesenteric arteries, active wall stresses (AWS), relaxation in response to acetylcholine and wall-tolumen ratio (W/L) were assessed. Western blotting and immunofluorescent staining of whole mount arterial preparations and two photon laser scanning microscopy (TPLSM) were performed to quantify eNOS and analyze its intracellular distribution.

Results: In 8 weeks old SHR treatments reduced SBP ((i) $123 \pm 1$ (ii) $164 \pm 2$ (iii) $155 \pm 3$ (iv) $156 \pm 3 \mathrm{mmHg} ; \mathrm{P}<0.05)$. Relaxation, contractile responses and vascular morphology remained unaffected irrespective of treatment. At 72 weeks, SBP was similar in all SHR groups ((i) 129 \pm 6 , (ii) $222 \pm 10$, (iii) $210 \pm 16$, (iv) $214 \pm 9 \mathrm{mmHg}$ ). Relaxation (-LogEC50: (i) $7.7 \pm 0.1$, (ii) $6.5 \pm 0.1$, (iii) $7.0 \pm 0.2$, (iv) $6.9 \pm 0.1$ ) and maximum AWS ((i) $50 \pm 4$ (ii) $48 \pm 7$ (iii) $126 \pm 12$ (iv) $109 \pm 10 \mathrm{~N} / \mathrm{m}^{2}$ ) were enhanced after treatments. W/L-ratio demonstrated hypertrophy ((i)0.10 \pm 0.01 (ii) $0.16 \pm 0.02$ (iii) $0.15 \pm 0.01$ (iv) $0.17 \pm 0.01)$. Untreated $S H R$ $(P<0.01)$, SHR-Los and SHR-Spiro $(P<0.05)$ showed less eNOS than WKY. In treated SHR eNOS was concentrated in a perinuclear endothelial cell compartment.

Conclusion: These findings demonstrate that transient prehypertensive RAAS blockade results in a long lasting and blood pressure independent improvement of arterial contractility and endothelium-dependent vasodilatation that persist in aging SHR. This might be associated with an intracellular redistribution of eNOS in the endothelial cell layer.
\end{abstract}




\section{Introduction}

It is well established that transient prehypertensive treatment of young spontaneously hypertensive rats (SHR) results in prolonged reduction of mean arterial pressure (MAP). The reduction persists after the withdrawal of treatment into adulthood. This has in particular been investigated for agents that suppress components of the renin-angiotensinaldosterone system (RAAS) $(103,104,108,111)$. The factors responsible for altering the blood pressure development of SHR remain unknown. Several authors suggest long-lasting influences on the feedback regulation of the RAAS $(104,105)$.

The mechanisms initiating and maintaining the elevated blood pressure of SHR are debated. It is generally believed that an increased responsiveness to vasoconstrictor stimuli and alterations of arterial structure are involved. Intraluminal pressure and constrictor stimuli are persistently increased in SHR. Thereby they induce and maintain arterial remodelling and prolonged vasoconstriction (111). The components of the RAAS belong to these contractile stimuli which show lifelong activity (152). Angiotensin II and aldosterone, the active components of the RAAS, in turn further complicate hypertension and cardiovascular pathologies by enhancing blood pressure and cardiovascular end-organ damage $(125,153$, 154).

Endothelial dysfunction can be considered as one of the long-term consequences of hypertension besides arterial remodelling and increased contractility (152, 155). Pharmacological interventions targeting RAAS improve the endothelium-dependent relaxant responses in aortas and mesenteric arteries of SHR. This has been shown for an ACEinhibitor, AT1-antagonist and an aldosterone antagonist $(153,156)$. These agents have also been shown to reduce blood pressure and end-organ damage $(130,156,157)$.

Effects of transient prehypertensive treatment in this context are largely unknown. Blood pressure reduction is known to persist into adulthood (36 week of age). This is associated with cardiac and renal protection $(105,108)$. Despite the prolonged blood pressure lowering effect, arterial hypertrophy remains unaffected (155). Data on vascular reactivity after transient prehypertensive treatment are missing. Long-term effects of this treatment after restoration of hypertension at an even older age (e.g. 72 weeks of age) are also missing. Therefore it remains unclear whether transient prehypertensive treatment has effects on arterial structure and reactivity and to what extent they are related. Theoretically, arterial effects could be i) related to the direct antihypertenive action during prehypertensive treatment, or ii) related to the prolonged antihypertensive action, or iii) could even be blood 
pressure independent. The latter would imply effects of the RAAS modulation during early stages of life, on long term mechanisms such as aging.

The purpose of the present study was to investigate: (1) whether prehypertensive RAAS blockade has blood pressure related or unrelated effects on arterial morphology or reactivity that persist up to advanced age and (2) whether effects differ between AT1 and aldosterone antagonism in SHR.

\section{Methods}

\section{Experimental set-up}

Four weeks old male WKY $(n=9)$ and SHR $(n=45)$ were obtained from Charles River (Landgraaf, The Netherlands). The rats were fed a normal sodium diet and had free access to water. All experiments were approved by the animal ethics committee of the Maastricht University and were performed in accordance with institutional guidelines.

At 4 weeks of age, rats were randomly assigned to either the treatment (SHR-Los, losartan, $20 \mathrm{mg} / \mathrm{kg}$ per day, SHR-Spiro, spironolactone, $0.5 \mathrm{mg} / \mathrm{kg}$ per day) or the control groups (SHR, WKY tap water). Treatments were performed continuously from week 4 to 8 of age using subcutaneously implanted minipumps. At 8 weeks systolic blood pressure was measured in SHR ( $n=6$ per group), using an intra-arterial catheter under pentobarbital anaesthesia. Thereafter from each rat 2 first order mesenteric artery branches were used for in vitro investigation. The remaining rats were housed under controlled conditions of temperature $\left(21^{\circ} \mathrm{C}\right)$ and light (12-hour light/dark cycle, $7 \mathrm{AM}$ to $\left.7 \mathrm{PM}\right)$. In aged rats blood pressure was measured and small mesenteric arteries were harvested using similar techniques as in 8 weeks old rats.

\section{Arterial reactivity}

Two first order mesenteric artery segments were analyzed for each experimental animal. The segments were mounted (steel wires, diameter $40 \mu \mathrm{m}$ ) in a myograph organ bath (model 610M, Danish Myotechnology by J.P. Trading, Denmark) for isometric tension measurements. Organ baths were filled with a Krebs-Ringer bicarbonate buffer, maintained at $37^{\circ} \mathrm{C}$ and aerated with $95 \% \mathrm{O}_{2}$ and $5 \% \mathrm{CO}_{2}$. The arterial segments were stretched until maximal contractile responses to $40 \mathrm{mM}$ potassium solution $\left(\mathrm{K}^{+}\right)$were obtained; this was considered the optimal diameter for the remainder of the study. Contractile responses were further obtained with noradrenaline (NA, $10^{-8}-10^{-4} \mathrm{M}$ ) and electrical field stimulation (EFS, 
$0.1-16 \mathrm{~Hz}$ ) of the perivascular autonomic nerves. Relaxing effects were investigated during contraction with $40 \mathrm{mM} \mathrm{K}$ using acetylcholine $\left(\mathrm{ACH}, 10^{-8}\right.$ to $10^{-5} \mathrm{M}$, half-log units) and sodium nitroprusside (SNP, $10^{-8}-10^{-5} \mathrm{M}$ ).

\section{Drugs and solutions}

Krebs-Ringer bicarbonate buffer contained the following (in $\mathrm{mM}$ ): $\mathrm{NaCl}, 118.5 ; \mathrm{MgSO}_{4} \cdot 7 \mathrm{H}_{2} \mathrm{O}$, 1.2; $\mathrm{KH}_{2} \mathrm{PO}_{4}, 1.2 ; \mathrm{NaHCO}_{3}, 25.0 ; \mathrm{CaCl}_{2}$, 2.5; glucose, 5.5. Solutions containing different concentrations of $\mathrm{K}^{+}$were prepared by replacing part of the $\mathrm{NaCl}$ by an equimolar amount of $\mathrm{KCl}$. Noradrenaline was obtained from Sigma Chemical Co. (St. Louis, MO, U.S.A.), ACH chloride from Janssen Chimica (Beersen, Belgium), and SNP from Acros (Geel, Belgium). All agents were dissolved in distilled water.

\section{Vascular morphology of mesenteric artery}

After in vitro experimentation, arteries were fixed overnight at their optimal diameter by replacing the organ-bath solution with phosphate-buffered $(\mathrm{pH} 7.4)$ formaldehyde $(4 \%)$. The next day, arteries were transferred to $70 \%$ ethanol and processed for histological examination. Arterial segments were embedded in paraffin and Lawson solution (Boom B.V. Meppel, The Netherlands) was used to stain the internal and external elastic laminae in 4$\mu \mathrm{m}$-thick cross-sections. To visualize the elastic laminae, a Zeiss Axioscope (Zeiss, Jena, Germany) and a standard CCD camera were used (Stemmer, Munich, Germany). Media cross-sectional area (CSA) was calculated by subtracting the area enclosed by the internal elastic lamina from the area enclosed by the media-adventitial border using commercial software (JAVA 1.21; Jandel Scientific, Corte Madera, CA, U.S.A.). Lumen diameter (D) was calculated from the internal circumference assuming a circular cross-section. Media thickness (Mt) was derived from the CSA and D measurements.

\section{ENOS staining / Two-Photon Laser Scanning Microscopy (TPLSM)}

Formalin fixed whole vessel segments were pretreated with Triton-PBS buffer. Therafter the whole segment was incubated with monoclonal eNOS mouse antibody (N30020, Transduction Laboratories, Exeter, UK), followed by a fluorescein isothiocyanate (FITC) labeled rabbit anti-mouse antibody (Alexa 488, Molecular Probes, Leiden, The Netherlands). After eNOS antibody treatment, vessels were imbedded in $1.5 \%$ agarose gel and co-stained with cell nuclei markers SYTO 41 (final concentration $=2.0 \mu \mathrm{M}$ ) or propidium iodide (final 
concentration $=1.5 \mu \mathrm{M})$. Both probes were obtained from Molecular Probes, Leiden, the Netherlands and dissolved in Hanks Buffered Saline Solution (HBSS, pH 7.4) containing (mmol/l): $137 \mathrm{NaCl}, 14.9$ HEPES, 5.5 glucose, $4.7 \mathrm{KCl}, 2.5 \mathrm{CaCl}_{2}, 1.2 \mathrm{KH}_{2} \mathrm{PO}_{4}$, and 1.2 $\mathrm{MgSO}_{4}$.

Imaging was performed on a Nikon E600FN microscope (Nikon Corporation, Japan), coupled to a standard Biorad 2100 MP multiphoton system (Biorad, Hemel Hempstead, GB). The excitation source was a pulsed Ti:sapphire laser (Spectra Physics Tsunami, Mountain View, USA) tuned and mode-locked at $800 \mathrm{~nm}$. Laser light reached the sample through the microscope objective $(60 \times$ water dipping, numerical aperture 1.0). Maximum field of view was $206 \mu \mathrm{m}$ * $206 \mu \mathrm{m}$. Further magnification with higher resolution was achieved by optical zoom in the scan head.

Three photo multiplier tubes (PMT) were used to detect the emitted fluorescent signals (158). For imaging of the fluorescent markers, PMT's were tuned corresponding to (parts of) the emission spectra of the used fluorescent markers: SYTO 41 450-470-nm (PMT I); FITC labeled eNOS antibody, 500 - $550 \mathrm{~nm}$ (PMT II); propidium iodide (PI) 570 - 700nm (PMT III). Laser power was kept as low as possible to prevent photochemical and thermal damage to the sample. Imaging speed was $0.1 \mathrm{~Hz}$ with a pixel dwell time of $39 \mu \mathrm{s}$, or $0.3 \mathrm{~Hz}$ with a pixel dwell time of $12 \mu$ s combined with Kalman filtering ( $n=3$ cycles) for noise reduction. From all PMT's, separate images of 512 * 512 pixels were obtained (color coded blue, green and red) and combined into a single image. All images were recorded in the $x y$-plane and are shown without further image processing. 3D-reconstructions of the scanned volume were obtained from z-stacks (a series of xy-images at successive depths; $z$-step $0.45 \mu \mathrm{m}$ or $0.6 \mu \mathrm{m})$. For $3 \mathrm{D}$ images additional processing was performed using the Image-pro plus 6.0 software and $3 \mathrm{D}$-constructor 5.1 packages (Media Cybernetics, USA) for the reconstruction of 3D-images.

\section{Western Blotting}

Aorta from adult untreated and treated SHR ( $n=5 /$ group) was prepared and western blotting performed. Briefly, membranes were incubated with rabbit monoclonal antibody against eNOS ( $\approx 135 \mathrm{kDa}$ ) diluted 1:2500, in washing solution at room temperature for 1 hour. The membranes were then washed, incubated with anti-rabbit horseradish peroxidaseconjugated second antibody 1:2000 for 1 hour at room temperature, and washed extensively. Membranes were incubated with Chemiluminescence Blotting Substrate 
(Boehringer Mannheim), according to the manufacturer's protocol, and exposed to film that was immediately developed. The film was detected with a Zeiss Axioscope (Zeiss, Jena, Germany) and a standard CCD camera (Stemmer, Munich, Germany) and saved to a computer. Band intensity was measured by computer analysis, using the LeicaQwin program.

\section{Statistics}

Contractile responses are expressed as active wall tension (increase in force divided by twice the length of the vessel segment, $\mathrm{N} / \mathrm{m}$ ) and as active wall stress (active wall tension divided by media thickness, $\mathrm{N} / \mathrm{m}^{2}$ ) to correct for differences in wall thickness. Data of WKY, SHR and transiently prehypertensive treated SHR were compared by ANOVA with Post-Hoc Dunnett's $t$ test. Data are expressed as mean \pm SEM. Differences with $P<0.05$ are considered statistically significant.

Table 3.1 Systolic blood pressure in 8 and 72 weeks old rats

\begin{tabular}{lllll}
\hline Age & WKY & SHR & SHR-LoS & SHR-Spiro \\
8 weeks & $123 \pm 4$ & $164 \pm 2^{*}$ & $155 \pm 4^{*}, \$$ & $156 \pm 2^{\star}, \$$ \\
72 weeks & $130 \pm 5$ & $222 \pm 9^{*}$ & $210 \pm 15^{*}$ & $214 \pm 9^{*}$ \\
\hline
\end{tabular}

SHR-Los: prehypertensively losartan treated SHR, SHR-Spiro: prehypertensively spironolactone treated SHR. * $P<0.001$ all SHR groups versus WKY; $\$ P<0.05$, prehypertensively treated SHR versus SHR.

\section{Results}

\section{Blood pressure}

Blood pressure results are summarized in Table1. In 8 week old rats, SBP was significantly increased in untreated SHR as compared to WKY. SHR treated from 4 to 8 weeks of age had significantly lower SBP than untreated SHR at 8 weeks of age. Comparable blood pressure lowering was obtained with losartan and spironolactone. In 72 weeks old animals (64 weeks after drug withdrawal) SHR had significantly elevated SBP as compared to WKY. Prehypertensively treated SHR showed comparable SBP as untreated SHR. 


\section{Morphological properties of small mesenteric arteries}

In 8 week old SHR, lumen diameter, media CSA, media thickness and wall-to-lumen ratio were comparable in untreated and prehypertensively treated SHR (Table 3.2). In 72 week old animals, SHR showed increased values as compared to WKY. Prehypertensively treated SHR did not show different values as compared to untreated SHR. Prehypertensive losartan treatment led to a slightly smaller W/L ratio than spironolactone treatment when compared to WKY (Table 3.2).

Table 3.2 Morphological properties of mesenteric small arteries in 8 and 72 weeks old rats

\begin{tabular}{llcccc}
\hline Age & & WKY & SHR & SHR-Los & SHR-Spiro \\
8 weeks & CSA $\left(\mu \mathrm{m}^{2}\right)$ & n.d & $5511 \pm 291$ & $5896 \pm 534$ & $6509 \pm 607$ \\
& Mt $(\mu \mathrm{m})$ & n.d & $8.2 \pm 0.4$ & $8.2 \pm 0.5$ & $8.8 \pm 0.6$ \\
& Diameter $(\mu \mathrm{m})$ & n.d. & $205 \pm 2.3$ & $219 \pm 7$ & $224 \pm 11$ \\
& W/L & n.d. & $0.08 \pm 0.01$ & $0.07 \pm 0.01$ & $0.08 \pm 0.01$ \\
72 weeks & CSA $\left(\mu^{2}\right)$ & $16909 \pm 496$ & $24812 \pm 2490^{\star}$ & $23476 \pm 1703^{\star}$ & $24496 \pm 1139^{\star}$ \\
& Mt $(\mu \mathrm{m})$ & $16.5 \pm 0.5$ & $23.6 \pm 2.0^{\star}$ & $24.7 \pm 2.6^{\star}$ & $24.4 \pm 1.5^{\star}$ \\
& Diameter $(\mu \mathrm{m})$ & $305 \pm 11$ & $296 \pm 13$ & $318 \pm 17$ & $290 \pm 6$ \\
& W/L & $0.11 \pm 0.01$ & $0.16 \pm 0.01^{\star \star}$ & $0.15 \pm 0.02^{\star}$ & $0.17 \pm 0.01^{\star \star}$ \\
\hline
\end{tabular}

SHR-Los: prehypertensively losartan treated SHR, SHR-Spiro: prehypertensively spironolactone treated SHR, CSA: media cross-sectional area, Mt: media thickness, W/L: wall-to-lumen ratio. ${ }^{*} P<0.05,{ }^{* *} P$ $<0.01$ all SHR groups versus WKY

\section{Contractile responses and active wall stress}

To assess receptor-dependent, receptor-independent and neurogenic contractile responses, isolated small mesenteric arteries of young and aged animals were treated with $\mathrm{K}^{+}, \mathrm{NA}$ and EFS, respectively (Table 3.3). In 8 week old SHR the responses to $K^{+}, N A$ and EFS were comparable in untreated and prehypertensively treated SHR. In 72 weeks old animals depolarisation lead to comparable contraction in untreated SHR and WKY. Prehypertensively treated SHR showed significantly increased contractile responses to depolarisation as compared to untreated SHR and WKY. Responses were accentuated in prehypertensively losartan-treated SHR. The AWS during depolarisation (correcting the AWT for alterations in media thickness) showed comparable values for untreated SHR and WKY. Prehypertensively treated SHR showed significantly increased AWS to depolarisation as compared to untreated SHR and WKY. Responses were similar in both prehypertensively treated SHR groups. 
Table 3.3 Reactivity of mesenteric small arteries of 72 weeks old rats

\begin{tabular}{|c|c|c|c|c|c|}
\hline & & WKY & $\overline{\text { SHR }}$ & SHR-Los & SHR-Spiro \\
\hline \multirow[t]{4}{*}{ AWT } & $\mathrm{K} \max (\mathrm{N} / \mathrm{m})$ & $.1 .7 \pm 0.1$ & $1.9 \pm 0.2$ & $3.1 \pm 0.3^{\star \star \star}, \$ \$$ & $2.6 \pm 0.2^{\star}$ \\
\hline & $N A \max (\mathrm{N} / \mathrm{m})$ & $4.6 \pm 0.1$ & $6.5 \pm 0.3^{* *}$ & $7.4 \pm 0.3^{\star \star \star}$ & $6.9 \pm 0.6^{\star \star \star}$ \\
\hline & $\mathrm{EFS} \max (\mathrm{N} / \mathrm{m})$ & $0.9 \pm 0.7$ & $3.8 \pm 0.5^{*}$ & $5.0 \pm 1.2^{* \star}$ & $5.0 \pm 1.0^{* *}$ \\
\hline & EFS max/NA max & $0.17 \pm 0.12$ & $0.65 \pm 0.07^{* * *}$ & $0.65 \pm 0.16^{\star \star \star}$ & $0.68 \pm 0.11^{* * *}$ \\
\hline \multirow[t]{2}{*}{ AWS } & $\mathrm{K} \max \left(\mathrm{N} / \mathrm{m}^{2}\right)$ & $50 \pm 4$ & $48 \pm 7$ & $126 \pm 12^{* * *}, \$ \$ \$$ & $109 \pm 10^{\star \star \star}, \$ \$ \$$ \\
\hline & $N A \max \left(\mathrm{N} / \mathrm{m}^{2}\right)$ & $265 \pm 9$ & $258 \pm 15$ & $319 \pm 12 *, \$, \#$ & $216 \pm 16$ \\
\hline
\end{tabular}

SHR-Los: prehypertensively losartan treated SHR, SHR-Spiro: prehypertensively spironolactone treated SHR, AWT: wall tension, AWS: wall stress, $\mathrm{K}$ max: maximal response to $40 \mathrm{mmol} / / \mathrm{K}^{+}$effective potassium dosage, NA max: maximal response to noradrenaline, EFS: electric field stimulation. ${ }^{*} P<0.05$, ${ }^{* *} P<0.01$, ${ }_{* \star \star} P<0.001$ all SHR groups versus WKY; $\$ P<0.05, \$ \$ P<0.01, \$ \$ \$ P<0.001$ prehypertensively treated SHR versus SHR; \# $P<0.001$ SHR-Los versus SHR-Spiro

In 72 week old animals NA and EFS led to larger contractile responses in untreated SHR as compared to WKY. Responses of prehypertensively treated SHR were non-significantly elevated as compared to SHR and significantly increased as compared to WKY. Responses were similar in both prehypertensively treated SHR groups. The AWS during NA (correcting the AWT for alterations in media thickness) showed comparable values for untreated SHR

Figure 3.1 Relaxation in small mesenteric arteries

A

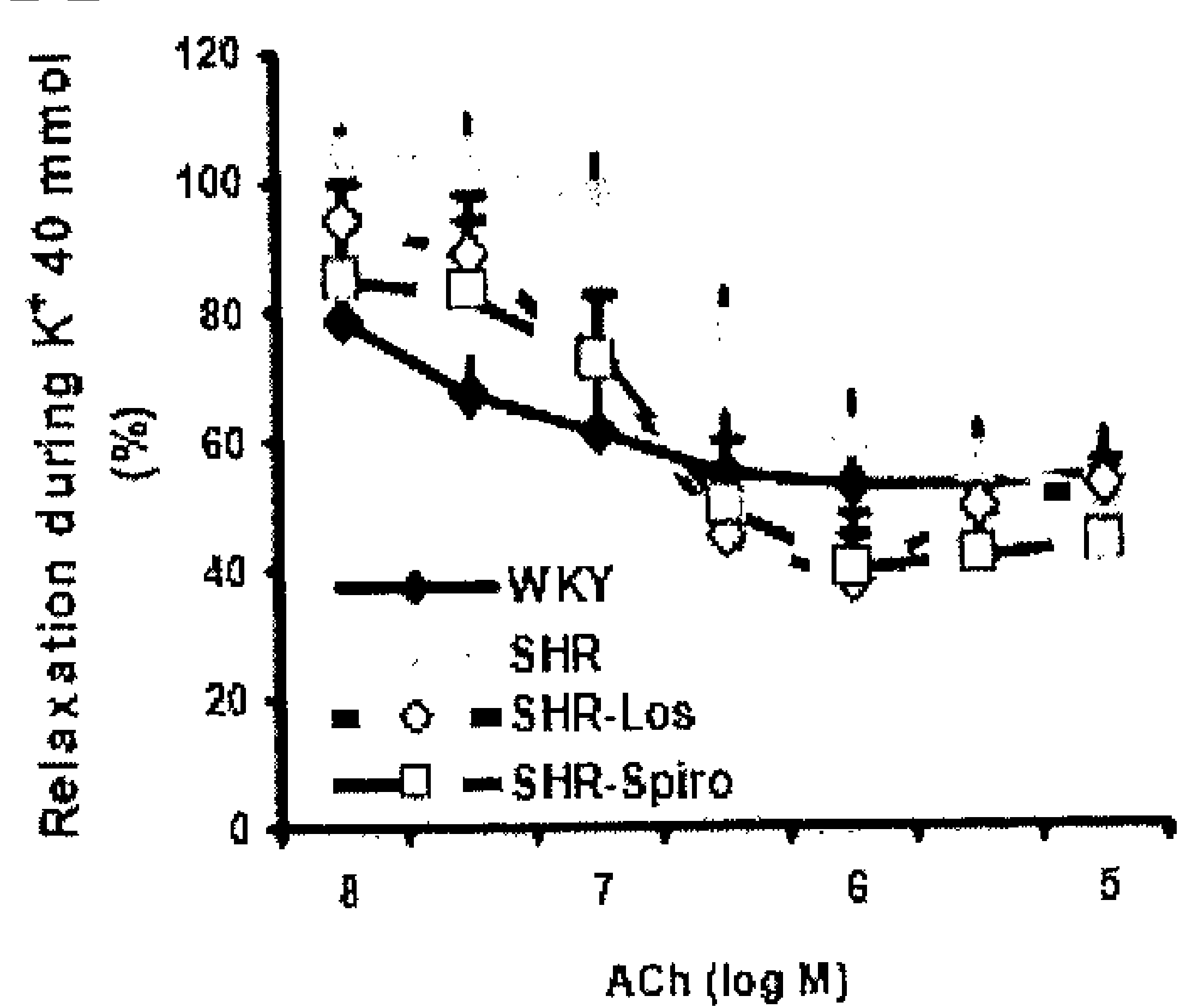

$\mathrm{B}$

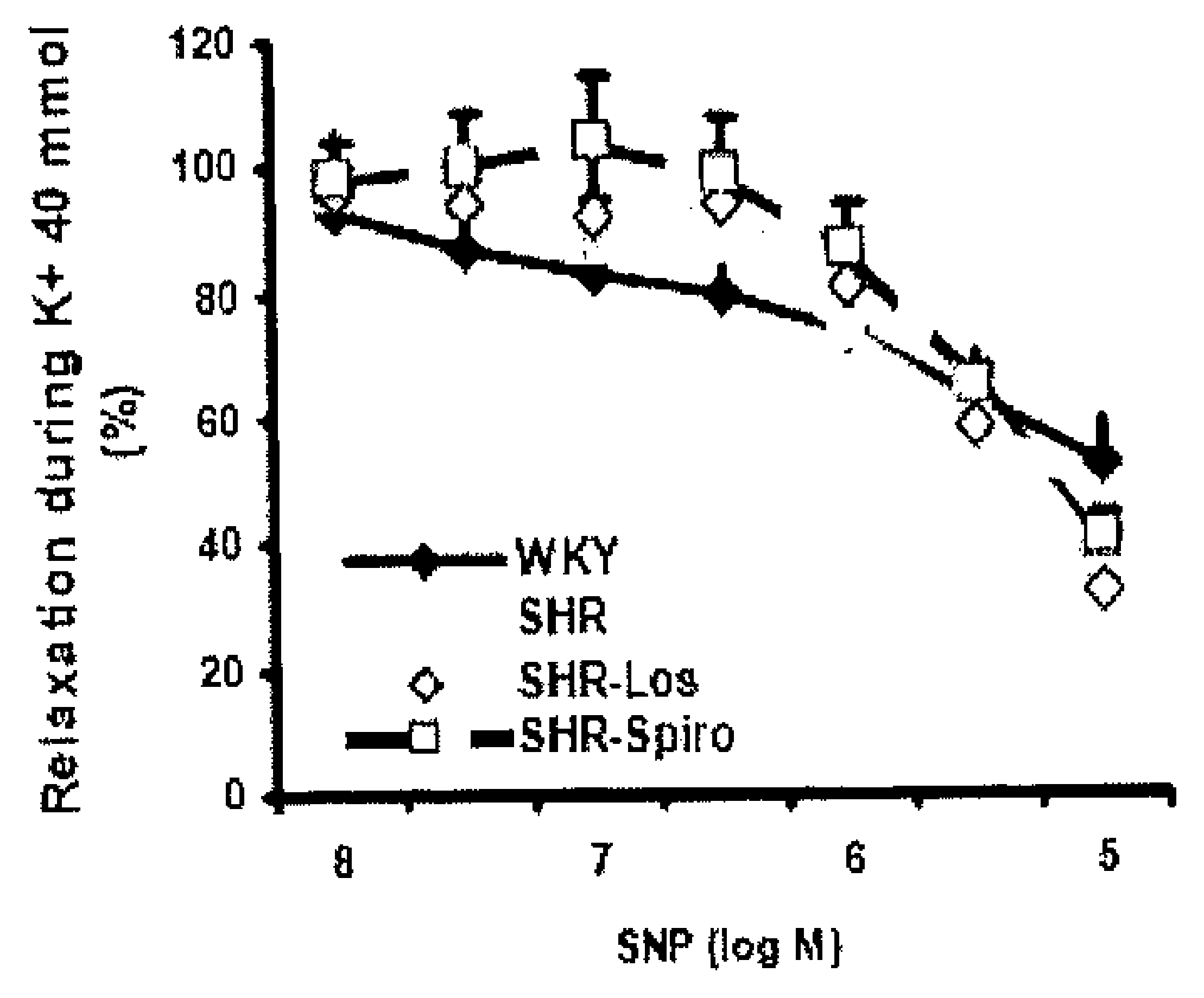

Endothelium-dependent $(A)$ and independent $(B)$ relaxation in small mesenteric arteries of 72 weeks old untreated WKY, SHR and prehypertensively losartan or spironolactone treated SHR. (A) The concentration of $A C h$ needed to half maximally relax potassium precontracted small mesenteric arteries

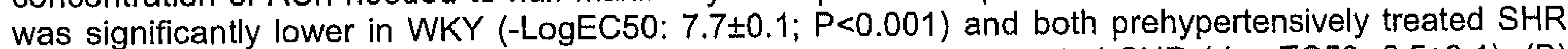
groups (-LogEC50: $7.0 \pm 0.2,6.9 \pm 0.1 ; \mathrm{P}<0.05$ ) as compared to untreated SHR (-LogEC50: 6.5 \pm 0.1 ). (B) Administration of the NO donor SNP resulted in comparable relaxing responses in all groups. 
and WKY. Prehypertensively losartan-treated SHR showed significantly increased AWS as compared to untreated SHR, WKY and in particular SHR-Spiro. The ratio of EFSmax/NAmax as an estimate of sympathetic neuroeffector function in untreated SHR was significantly increased as compared to WKY. Prehypertensively treated SHR showed comparable ratios as untreated SHR. Ratios were similar in both prehypertensively treated SHR groups.

\section{Relaxing responses}

In 8 weeks old SHR the relaxing responses of ACh and SNP during $\mathrm{K}^{+}$-induced contraction were comparable in untreated and prehypertensively treated SHR (data not shown). In 72 weeks old animals endothelium-dependent relaxation was significantly reduced in SHR as compared to WKY (Figure 3.1; $P<0.001$ ). Prehypertensively treated SHR showed significantly ameliorated endothelium-dependent relaxation as compared to untreated SHR $(P<0.05)$. Responses were similar in both prehypertensively treated SHR groups. The endothelium-independent relaxant agent SNP produced similar relaxations irrespective of strain and prehypertensive treatment.

Figure 3.2 Western blotting of eNOS
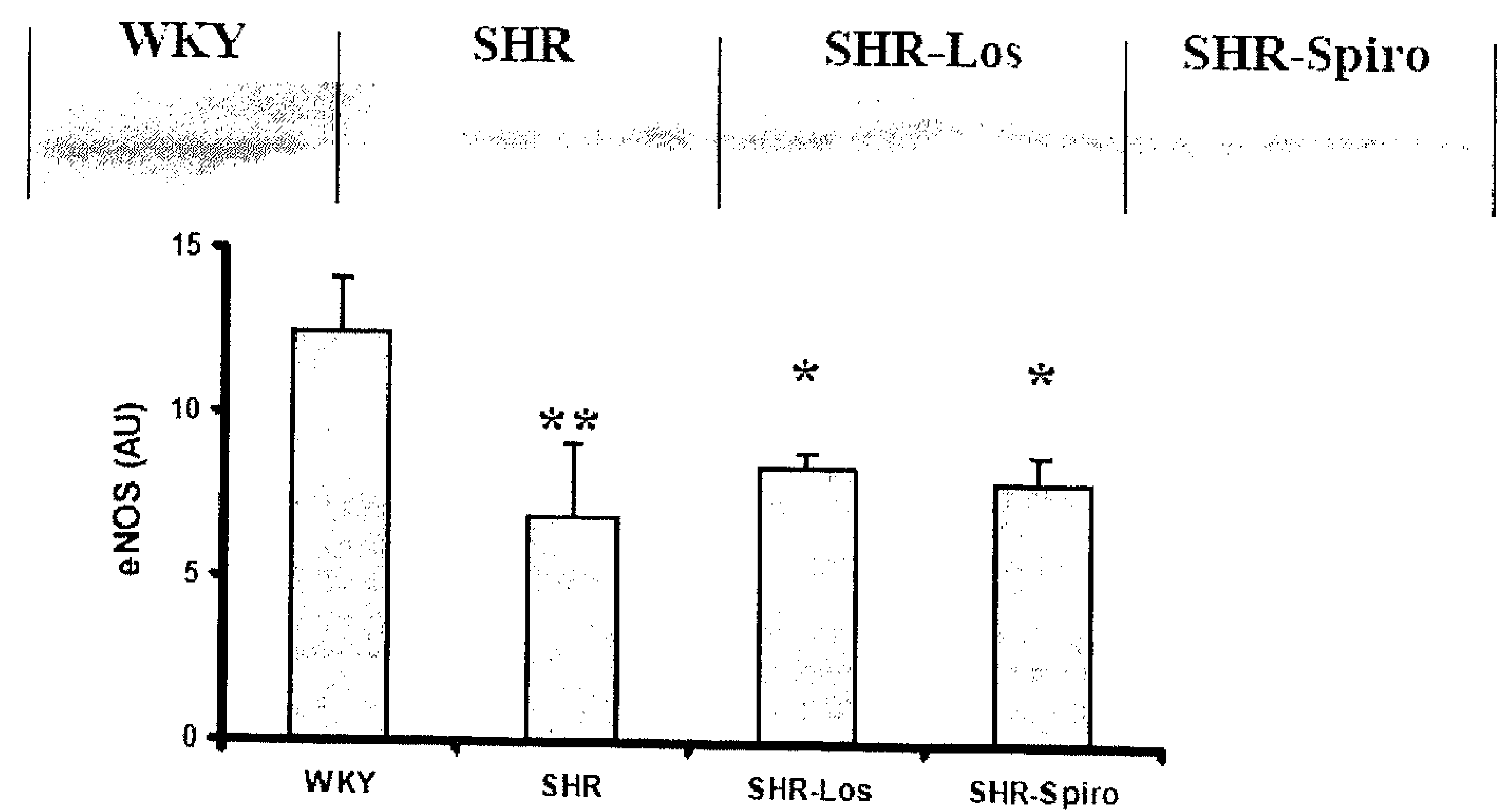

Western blotting of eNOS in aortic tissue of 72 weeks old WKY, untreated and prehypertensively treated SHR. Untreated SHR, prehypertensively losartan and spironolactone treated SHR demonstrate similar densities of eNOS. However significant differences to WKY are apparent between untreated $(P<0.01)$ and prehypertensively treated SHR $(P<0.05)$. The blot shows 2 WKY rats and 3 rats per SHR group. 


\section{Quantification of aortic eNOS}

Using aortic tissue eNOS density was quantified in untreated and treated SHR by Western blotting. Untreated SHR showed $50 \%$ less eNOS as compared to WKY $(P<0.01)$. SHR-Los and SHR-Spiro demonstrated also less eNOS than WKY and findings did not differ significantly from untreated SHR.

Figure 3.3 Two photon light scanning microscopy (TPLSM) of eNOS

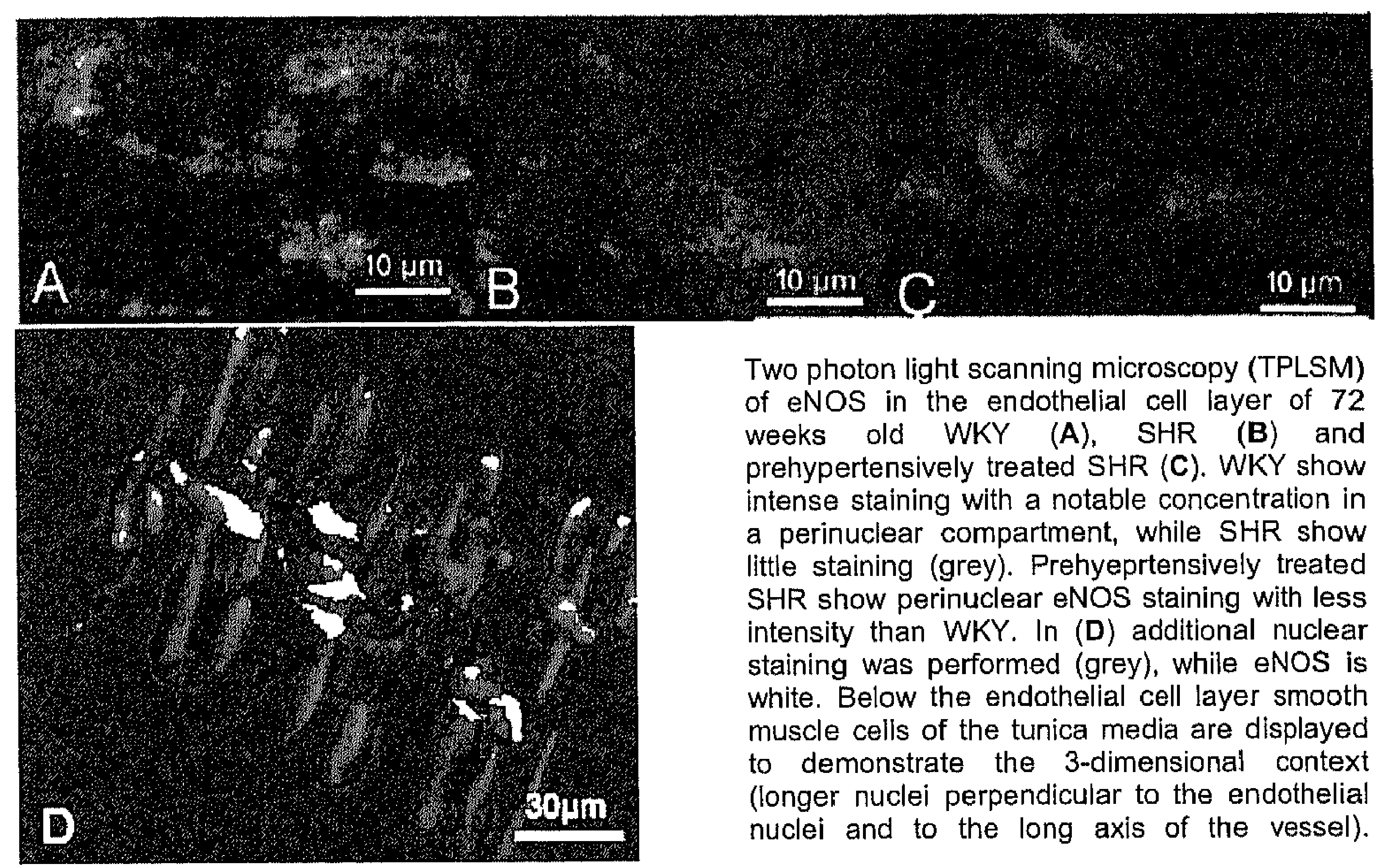

\section{Localization of eNOS in mesenteric arteries}

Whole vessel segments were investigated for eNOS density and intracellular distribution using two photon laser scanning microscopy (Figure 3.3). In 72 week old SHR significantly less endothelial eNOS was apparent as compared to WKY and less endothelial cells were stained above the detection limit. In SHR the fluorescent signal was less intense, while in WKY the majority of the signal was localized in one intracellular area located on one side of the nucleus. Prehypertensively treated SHR showed similar amounts of eNOS as compared to untreated SHR. The amounts were similar in both prehypertensively treated SHR groups. In contrast to the blurred localization in untreated SHR, prehypertensively treated SHR 
displayed plasmalemmal and perinuclear distributions of eNOS that were comparable to that in WKY.

\section{Discussion}

This study demonstrates that short-term RAAS blockade in young SHR resulted in altered reactivity of small mesenteric arteries at 64 weeks after withdrawal of the treatment. The alterations consisted of increased vasoconstriction and reduced endothelial dysfunction and were not associated with a persistent reduction of arterial hypertrophy and blood pressure. Effects were largely similar after transient prehypertensive therapy with losartan or spironolactone. These findings suggest that early inhibition of the RAAS might lead to a long lasting resetting of this humoral axis that slows the deterioration of small artery function with aging.

Prehypertensive RAAS blockade was induced by losartan or spironolactone from 4 to 8 weeks of age. At the end of these treatments, blood pressure was reduced but contractile responses and endothelial dysfunction were not modified. These suggest that brief antihypertensive RAAS blockade in prehypertensive SHR had no immediate effects on vascular structure and reactivity despite significant blood pressure lowering. This was unexpected as prehypertensive SHR are considered to demonstrate increased RAAS mediated contraction, ANG-II receptor density and hypertrophic remodeling $(159,160)$. This might be explained by the short duration of the treatment and by the relatively small blood pressure lowering (155).

We $(161)$ and others $(104,108)$ demonstrated previously that blood pressure lowering after prehypertensive treatment persists into adulthood (i.e. 36 weeks of age). In contrast to earlier studies that were terminated at this age we continued to monitor blood pressure and observed a restoration of hypertension at later stages of life (161). This is supported by our present results that at 72 weeks of age, blood pressure did no longer differ between prehypertensively treated and untreated SHR. This demonstrates that the blood pressure lowering effects of this treatment strategy was not life-long. The similar blood pressure values between the three 72 weeks old SHR groups lead us to conclude that the arterial changes observed in the old SHR treated at young age were not the direct consequence of a lower blood pressure $(152,155)$. 
In line with the absence of a blood pressure lowering effect, small artery structure did not differ between 72 weeks old SHR, prehypertensively treated or not with losartan or spironolactone. In contrast to these findings prehypertensive treatment resulted in increased maximal contractile responses to all stimuli investigated. When the thickness of the tunica media was taken into account, differences between old SHR with and without prehypertensive treatment persisted. This indicates that prehypertensive RAAS blockade resulted in increased contractility of the arterial smooth muscle in old SHR which was more marked after prehypertensive treatment with losartan than spironolactone. Whether this reflects a predominantly AT1R dependent alteration or rather a dosage phenomenon remains unclear. Recently Demirci et al. described a reduction of contractile responses during aging of SHR which was partially antagonized by continuous losartan treatment (162). Our data now suggest that not only continuous but also transient prehypertensive RAAS blockade might decelerate the loss of arterial contractility with aging in a blood pressure independent manner. Nakaya et al. proposed a role for resetting of the RAAS in the prevention of the premature aging of the kidney (105).

Unlike for smooth muscle, neuroeffector mechanisms did not seem to be modified by the prehypertensive treatments. While the ratio of maximal responses to nerve stimulation and noradrenaline was markedly enhanced in SHR compared to WKY, this functional index of sympathetic nerve density did not differ between untreated and prehypertensively treated SHR. Periarterial innervation, which in the rat is established during the first 2 weeks of life, thus seems not to be persistently altered by RAAS blockade between 4 and 8 weeks of age (163).

With respect to the endothelium we recorded relaxing responses to acetylcholine during $\mathrm{K}^{+}$induced contraction in order to concentrate on nitric oxide (NO) in the absence of influences of endothelium-derived hyperpolarizing factor. At 72 weeks of age these responses were ameliorated in prehypertensively treated SHR as compared to untreated SHR. Thus, not only contractile responses but also endothelium-dependent relaxing responses were improved by this treatment strategy in a blood pressure independent manner. Reversal of decreased bioavailability or synthesis of endothelium-derived nitric oxide NO, or of the dysfunction of vascular smooth muscle in aging hypertensive animals, might contribute. The latter possibility can be dismissed by the finding that SNP, a NO donor, produced equal degree of relaxations in SHR small mesenteric arteries, irrespective of treatment. Losartan and spironolactone both inhibit $\mathrm{NAD}(\mathrm{P}) \mathrm{H}$ oxidase-mediated $\mathrm{O}_{2}^{-}$ 
synthesis and enhance antioxidant superoxide dismutase activity in the small mesenteric arteries $(164,165)$. As a consequence, this increases NO bioavailability $(166,167)$. We therefore suggest that the beneficial effects of prehypertensive treatments were at least partially mediated by enhanced endothelial supply of NO.

To substantiate that the enhanced relaxing responses in prehypertensively treated SHR were related to the synthesis of NO, we investigated eNOS in aortic tissue. Using Western blotting we demonstrated that the concentration of eNOS was reduced in untreated SHR as compared to $W K Y$, and that this was not significantly altered by prehypertensive treatment. Thus other mechanisms could be involved in the enhanced relaxing responses. As NO synthesis depends on the intracellular distribution of eNOS (168) we investigated the endothelial cell layer of mesenteric arteries in untreated and treated SHR. As expected, the density of eNOS was larger in WKY as compared to SHR with a dense staining in a perinuclear region at one side of the nucleus, which is most likely the Golgi-apparatus (168). This perinuclear staining was absent in the endothelium of untreated SHR. Prehypertensively treated SHR demonstrated similar densities of eNOS as untreated SHR, thereby confirming our Western blot results. However, prehypertensively treated SHR demonstrated a perinuclear staining at the same perinuclear region as WKY, albeit at a lower concentration. The consequences of this eNOS localization remain uncertain. However, as eNOS distribution but not its amount is associated with endothelial function in untreated or prehypertensively treated SHR, respectively, the specific eNOS distribution might have functional consequences (169). Recent studies suggested that such a distribution can result from a decreased wall shear stress (168). Furthermore, the perinucelar eNOS distribution might attenuate peroxynitrite production as the therefore necessary NADPH oxidases are commonly distributed at the cell membrane (170). The combination of eNOS expression and intracellular distribution could result in the enhanced relaxing responses seen in prehypertensively treated SHR.

In summary, we demonstrated that prehypertensive RAAS inhibition resulted in small mesenteric arteries of old SHR in increased maximal contractile responses and ameliorated endothelial function as compared to untreated SHR. This process was blood pressure independent. As explanation we propose a long-lasting pharmacological resetting of the RAAS that culminates in deceleration of the premature aging of arterial function in SHR. 
CHAPTER FOUR:

Sustained renoprotection in SHR by transient prehypertensive losartan treatment: an effect of decelerated aging?

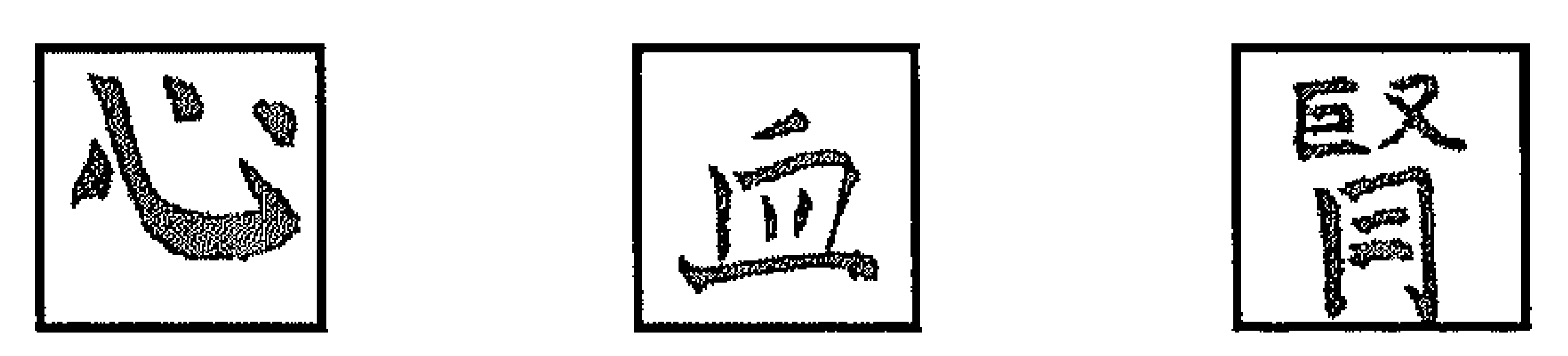

Marcus Baumann, Roger Bartholome, Carine Peutz-Kootstra, Jos F.M. Smits, Harry A.J. Struijker Boudier

Submitted 


\begin{abstract}
Background: Hypertensive target organ damage shows characteristics of accelerated cellturnover and aging. It remains uncertain whether blood pressure and aging are causally related, accelerated aging independently determines target organ damage or aging can be decelerated.

Methods: WKY (i) and SHR (ii) were prehypertensively (week 4-8) treated with losartan (iii) or hydralazine (iv) (20 and $4 \mathrm{mg} / \mathrm{kg} /$ day, respectively). These groups were investigated at weeks 8 and 12. At week 72 groups i-iii were investigated. At week 8 renal epithelial cell proliferation (proliferating cell nuclear antibody (PCNA)) was evaluated. At weeks 8 and 72 systolic blood pressure (SBP) was measured intra-arterially. At week 72 aging parameters as telomere length ( $\mathrm{qPCR}$ ) and proliferation marker were assessed. Renal damage was semiquantitatively (scale: $0-4$ ) assessed regarding parenchym (atrophy) and vasculature (wall to lumen ratio (W/L)).

Results: In 8 weeks old SHR treatments equipotently reduced SBP $(P<0.01)$ and except hydralazine reduced renal proliferation (PCNA: (i) $0.1 \pm 0.1$ (ii) $2.8 \pm 0.8$ (iii) $1.3 \pm 0.3$ (iv) $3.0 \pm 0.6$ cells $/ \mathrm{mm} 2$ ). At week 72 prehypertensive treatment increased renal telomere length as compared to untreated SHR (fold: (i) $1.0 \pm 0.2$, (ii) $0.1 \pm 0.1$, (iii) $0.3 \pm 0.1, P<0.01$ ) and reduced cell proliferation. Transiently treated SHR showed less tubular atrophy ((i) $0.5 \pm 0.2$, (ii) $1.8 \pm 0.3$, (iii) $1.1 \pm 0.2, P<0.01$ ). No vascular treatment effects were seen (W/L: (i) $4.4 \pm 0.7$, (ii) $8.3 \pm 1.5$, (iii) $8.9 \pm 1.4$ ).

Conclusion: These findings confirm that the aging process in SHR is accelerated and determines target organ damage. Renal aging is angiotensin-related and transient prehypertensive angiotensin inhibition decelerates aging up to advanced age.
\end{abstract}




\section{Introduction}

Observational studies demonstrated that pulse pressure increases with age (171). Therefore several authors suggested that essential hypertension represents an age-related disorder (172-174). This was underlined by Jeanclos et al., demonstrating that the level of pulse pressure was associated with individual aging parameters such as the telomere length (175).

Experimental studies in spontaneously hypertensive rats (SHR) revealed an accelerated cell-turnover of vascular smooth muscle cells as compared to normotensive rats (176). These findings were directly related to an increased cell proliferation at young age (173), which was counterbalanced by an increased apoptosis at later age (177). These findings further strengthened the idea that hypertension could be interpreted as disease of accelerated aging (173).

ANGII modifies cell proliferation and apoptosis by several pathways (178-181). TGF- $\beta$ represents a key mediator (182) leading to an activation of the G1 phase (183-186). Recent studies investigated the effect of chronic ACE-inhibition on aging defined as vascular and renal function $(162,187)$. They demonstrated that a blood pressure independent protection was evident after continuous ACE-inhibition. This sets a focus on the renin-angiotensin system (RAS) as potential mediator for the process of accelerated aging in hypertension. This idea is fuelled by the observation that the initial increase of cell proliferation in SHR coincides with the time window, in which in young SHR ANGII is over-activated $(96-98,188$, 189). Furthermore, transiently inhibiting ANGIl during this early time window leads to cardiovascular protection up to advanced age $(105,190)$. This leads us to the hypothesis that SHR are characterized by an ANGII-driven accelerated aging which independently of blood pressure determines target organ damage. We propose that a reduced action of angiotensin II should reduce the mitogenic effect and decelerate the aging process. To investigate this hypothesis we performed transient prehypertensive ANGII inhibition in young SHR.

\section{Methods}

Three weeks old male WKY and SHR were obtained from Charles River (Landgraaf, The Netherlands). The rats were fed a normal sodium diet and maintained on dry pellet diet and had free access to water. All experiments were approved by the animal ethics committee of the Maastricht University and were performed in accordance with institutional guidelines. 
Four weeks old WKY and SHR were given saline (placebo), losartan (SHR-Los: 20 $\mathrm{mg} / \mathrm{kg} /$ day), hydralazine (SHR-Hyd: $15 \mathrm{mg} / \mathrm{kg} / \mathrm{day}$ ), TGF- $\beta_{1}$-antibody (anti-TGF- $\beta_{1}: 1$ $\mu \mathrm{g} / \mathrm{kg}$.day, Biosource International, Camarillo, CA, USA) or control antibody (anti-lgG-k1: 1 $\mu \mathrm{g} / \mathrm{kg}$.day) for a period of four weeks using osmotic minipumps (Alzet 2004, Durect, CA, USA) that were implanted subcutaneously under isoflurane anaesthesia (1.5\%). Each group consisted of 7-9 animals. Blood pressure was measured in the conscious unrestrained state through a heparinized indwelling polyethylene catheter that was introduced into the left femoral artery two days before measurement at the age of 8 and 12 weeks of age (135). Cumulative dose-response curves for ANGII and phenylephrine were made via a heparinized indwelling polyethylene catheter that was introduced into the left femoral vein two days before measurement at 12 weeks of age. Plasma TGF- $\beta$ and ANGIl were measured using ELISA and RIA. Placebo treated WKY, SHR and SHR-Los were additionally investigated until 72 weeks of age. In these rats oxidative stress induced lipid peroxidation was longitudinally measured by quantification of thiobarbituric acid-reactive substances (TBARS) from 24 hour urine samples using HPLC (191).

Kidneys were harvested at 8 (all treatment groups) and 72 weeks (WKY, SHR and SHRLos) and were evaluated by H\&E, Sirius Red, proliferative cell nuclear antigen (PCNA) and a-smooth muscle actin (ASMA) as previously described by our group (141). Tubular atrophy was measured on H\&E by a blinded nephropathologist in a semi quantitative manner. Proliferating tubular cells were quantified as cells $/ \mathrm{mm}^{2}$. Interstitial collagen and perivascular collagen were determined from Sirius Red staining as previously described by our group (141). ASMA staining was used to determine the cross sectional area (CSA) of renal afferent arterioles as previously described by our group (141). All measurements were performed in blinded manner.

Renal cortical tissue was snap frozen and RNA isolated with an RNeasy Mini Kit (QIAGEN, Hilden, Germany). RNA was purified (Ultraspec-ll RNA, Biotec Laboratories, Houston, Tx, USA), quantified (Nanodrop ND-1000, WITEC, Switzerland) and transcribed into CDNA with Superscript III reverse-transcriptase, using $250 \mathrm{ng}$ of random primers (Invitrogen Life Technologies). Primers and probes against the selected genes of telomeres, cyclinD1, cdk4 pRb, p16, p21, p27 and type IV collagen were investigated. Renal cortical tissue was homogenized and protein concentration quantified. Common Western blot using phosphopRb (110kDa, 1:400, Cell Signaling Technology, Danvers, MA, USA, ) was performed. Data are given in mean $\pm S D$. We compared the groups with an one-way analysis of variance 
(ANOVA) including a post-hoc Bonferroni test. Anti-TGF- $\beta_{1}$ and control antibody were compared with Student's t-test. We considered $P<0.05$ to be significant.

Figure 4.1 Cell cycle progression in SHR is ANGIl, but not blood pressure dependent.

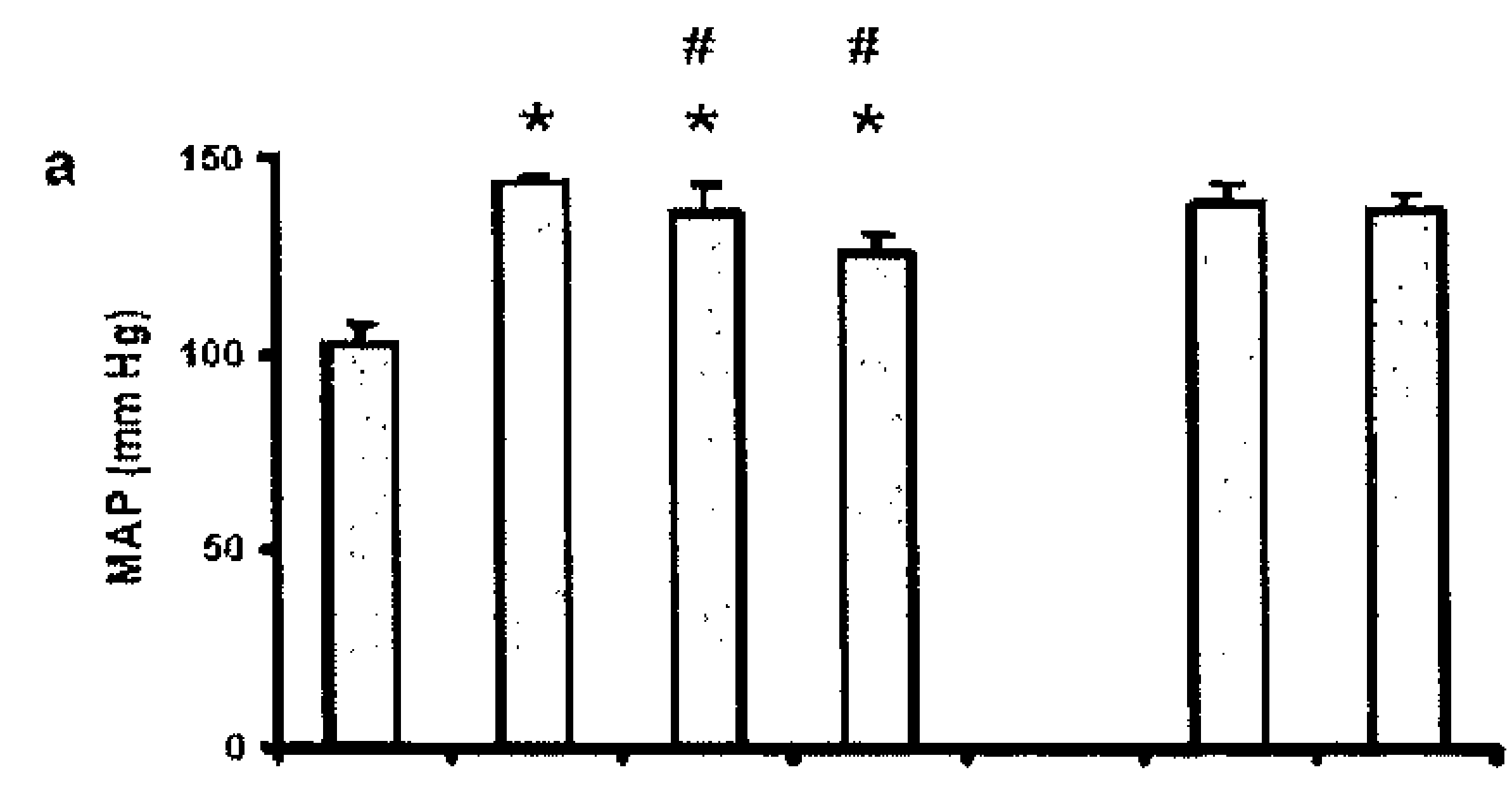

b
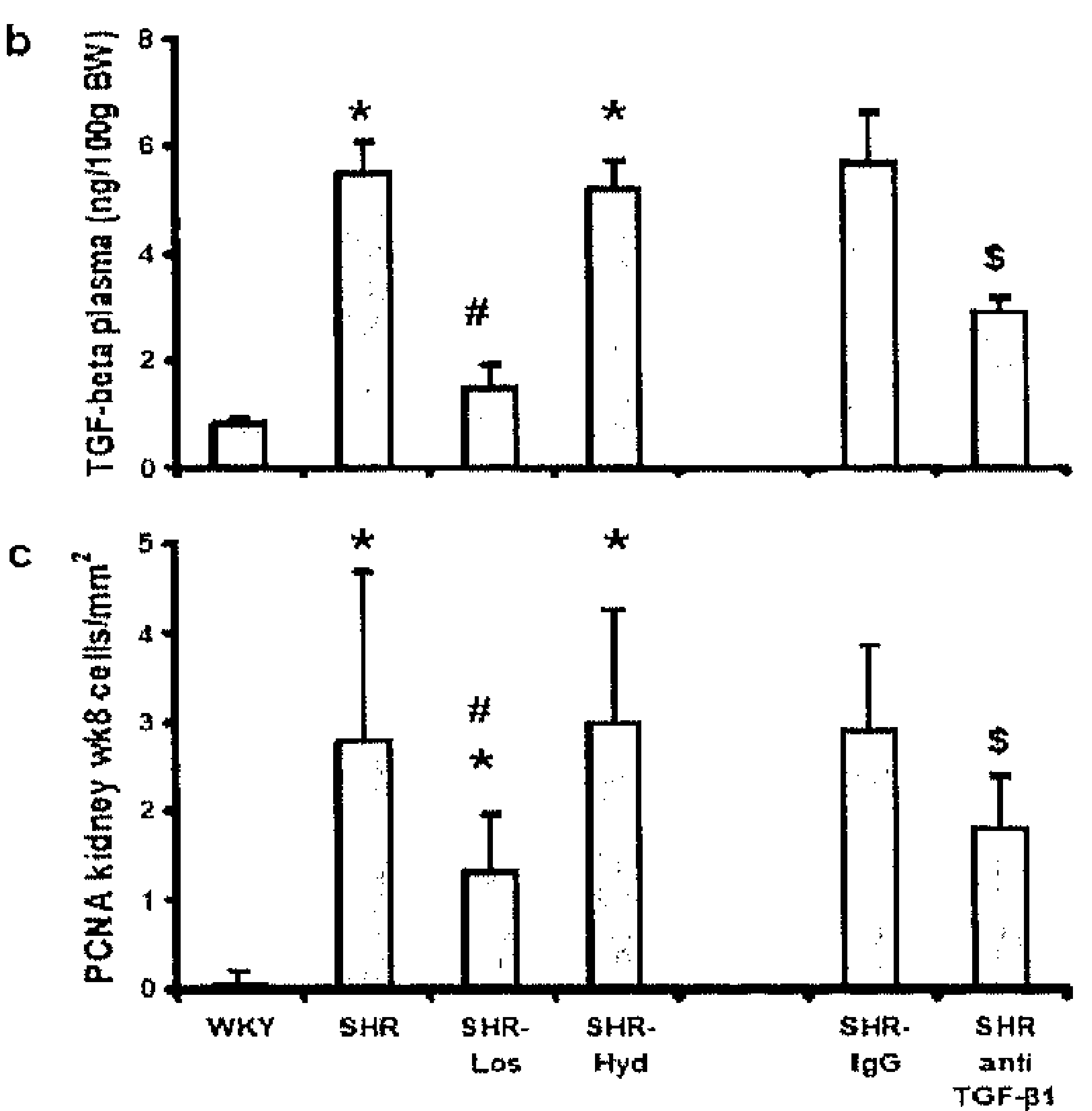

(A) Intra-arterial blood pressure measurements demonstrate blood pressure reduction in both antihypertensive treatment groups without reaching normotensive values. TGF- $\beta_{1}$ antibody treatment showed no blood pressure effect. (B) TGF- $\beta_{1}$ plasma levels were reduced in WKY and in losartan and anti TGF- $\beta_{1}$ treated SHR. (C) Proliferating renal tubular cells were visualized using proliferate cell nuclear antigen (PCNA) immuno-histochemistry and were quantified per area. ANGII antagonism and TGF- $\beta_{1}$ antibodies but not hydralazine treatment reduced proliferation of tubular cells. ${ }^{*} P<0.05$ vs. WKY, $\# P<0.05$ SHR-Los vs. SHR, $\$ P<0.05$ anti-TGF- $\beta_{1}$ vs. control antibody.

\section{Results}

As shown in Figure 1A, mean arterial pressure (MAP) was significantly reduced by both antihypertensive treatments as compared to untreated SHR $(P<0.05)$. This effect was not apparent after anti-TGF- $\beta_{1}$ treatment as compared to the placebo treatment with control IgG antibody (MAP (mmHg): WKY 103 \pm 1 , SHR 144 \pm 2 , SHR-Los 135 \pm 3 , SHR-Hyd 126 \pm 2 , SHR- 
control antibody 139 \pm 2 , SHR-anti TGF- $\beta$ 137 \pm 1 , Figure 4.1A). TGF- $\beta_{1}$ plasma levels were highest in SHR. Losartan but not hydralazine significantly reduced TGF- $\beta_{1}$ plasma level to WKY level as anti-TGF- $\beta_{1}$ treatment did (TGF- $\beta_{1}$ (ng/100g body weight): WKY $0.8 \pm 0.2$, SHR

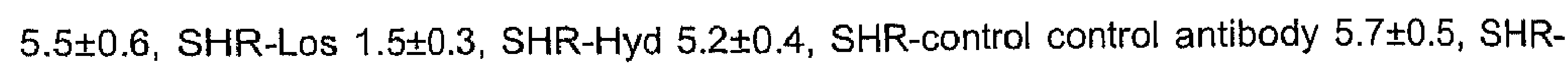
anti TGF- $\beta$ : $2.9 \pm 0.2$, Figure 4.1B). To investigate whether blood pressure or TGF- $\beta_{1}$ levels correspond with tubular cell proliferation in young SHR we quantified renal proliferating cells. The amount of proliferating tubular cells was highest in SHR. RAAS-antagonism significantly attenuated the amount as anti-TGF- $\beta_{1}$ treatment did. In contrast, the vasodilator hydralazine did not effect the rate of tubular cell proliferation (PCNA (cells $/ \mathrm{mm}^{2}$ ): WKY $0.1 \pm 0.1$, SHR 2.8 \pm 0.7 , SHR-Los 1.3 \pm 0.3 , SHR-Hyd 3.0 \pm 0.6 , SHR-control antibody $2.9 \pm 0.3$, SHR-anti TGFB $1.8 \pm 0.4$, Figure $4.1 \mathrm{C}$ ). We next investigated whether the reduced cell proliferation sustained after drug withdrawal in SHR-Los up to advanced age. Therefore, WKY, SHR and SHR-Los up were followed until 72 weeks of age. The SHR-Hyd group was not followed up until 72 weeks of age as they did not show any reduction of cell proliferation under treatment at 8 weeks of age. Tubular cell proliferation remained highest in untreated SHR and was significantly reduced by prehypertensive losartan treatment (PCNA (cells $/ \mathrm{mm}^{2}$ ): WKY

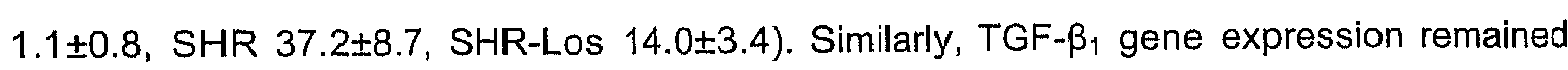
attenuated in transient prehypertensively treated SHR (TGF- $\beta_{1}$ (fold): WKY 1.0 0.1 , SHR $1.5 \pm 0.3$, SHR-Los 0.5 \pm 0.2 , Figure 4.2A-B).

As enhanced cell proliferation in hypertension has been attributed to an increased cell cycle progression during the early $\mathrm{G} 1$ phase (183), we investigated the major components of the

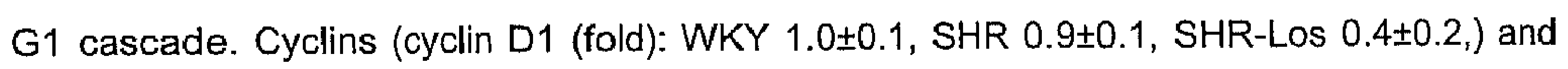
cyclin-dependent kinases (cdk 4 (fold): WKY 1.0 0.1 , SHR 3.0 \pm 0.3 , SHR-Los $0.6 \pm 0.1$ ) which form holoenzymes phosphorylating the retinoblastoma tumor suppression protein ( $\mathrm{pRb}$ ) were markedly reduced by the treatment (Figure 4.2C-D). The phorpshorylation of pRb was markedly reduced by the treatment as shown by Western blotting (phosphorylated $\mathrm{pRB}$

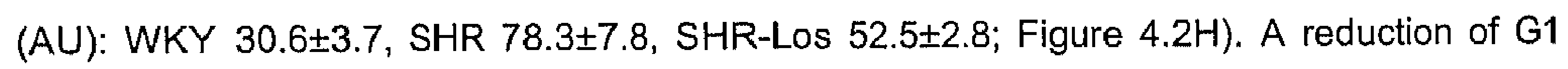
activity can be mediated by enhanced expression of inhibitory genes such as p16ink, p21 and p27 $(184,192)$. We demonstrate that the inhibitory genes were not increased by the

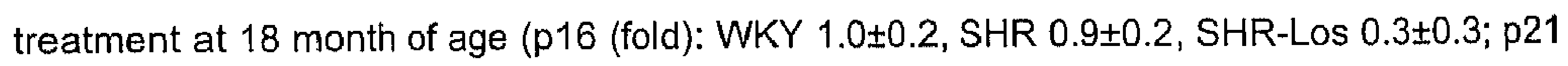

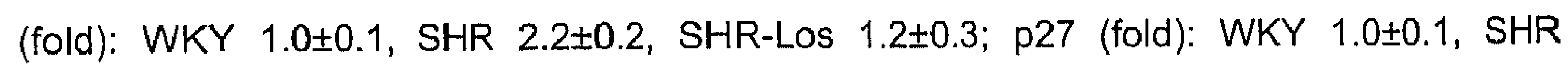
$1.7 \pm 0.4$, SHR-Los $0.6 \pm 0.2$ ). 
Figure 4.2 Cell proliferation and G1 signaling remained reduced after transient losartan treatment
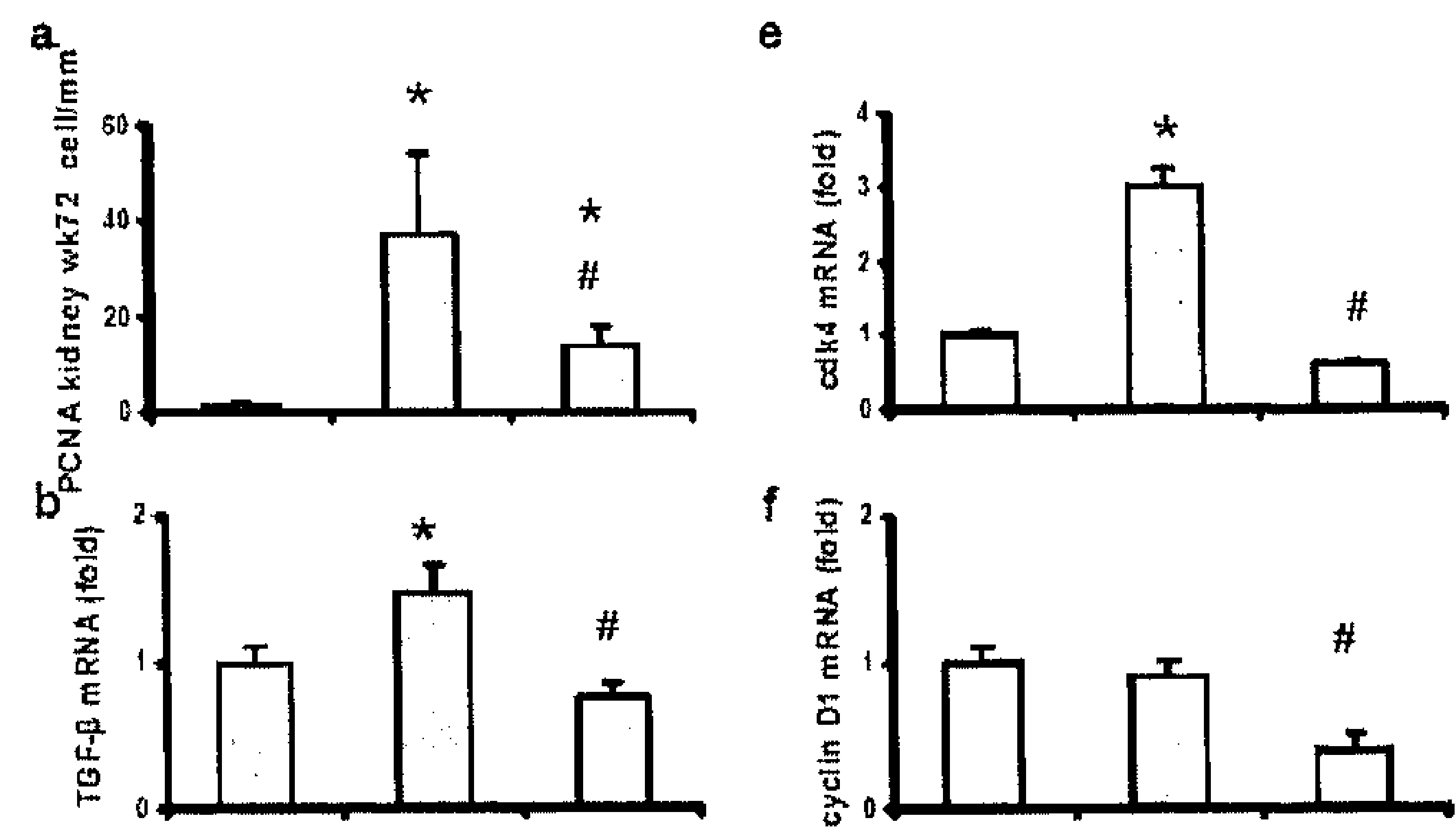

$c$

g
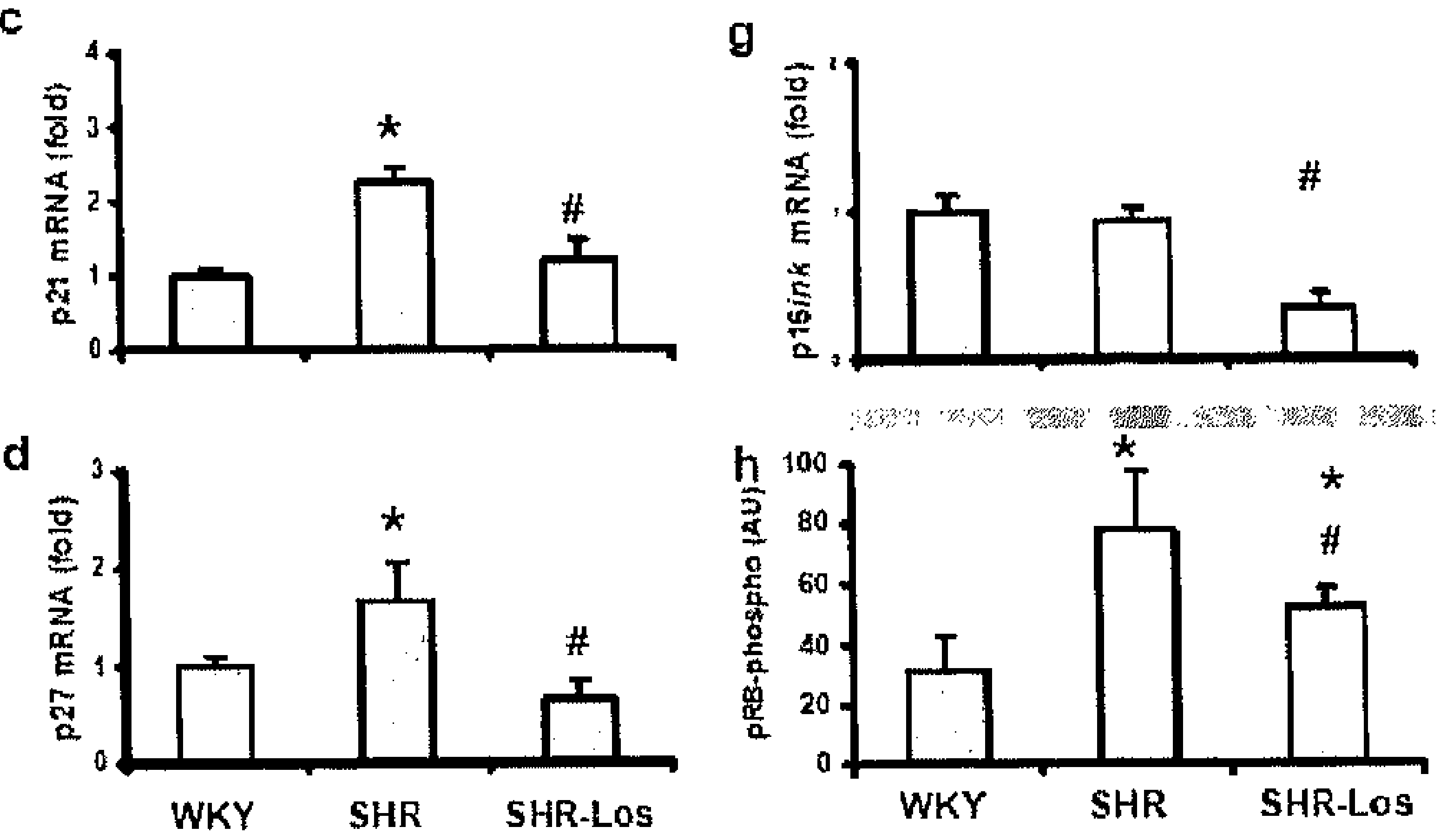

Cell cycle progression after transient ANGII inhibition remained lower until advanced age. (A) Proliferating renal tubular cells were visualized using immunohistochemistry. Positive cells were quantified per area. After 64 weeks of drug withdrawal in losartan treated SHR, tubular proliferation remained decreased by $50 \%$ as compared to untreated SHR. (B) TGF- $\beta_{1}$ gene expression was in previously treated SHR reduced as compared to placebo treated SHR and WKY. The same pattern was observed for (C) cyclin D1. (D) Prehypertensive treatment reduced the 3-fold enhanced cdk4-expression in SHR to normotensive level. (E-G) Expressions of inhibitory genes of the holoenzyme cyclin D/cdk4 were not activated by the transient treatment. $(H)$ The reduced gene expression of cyclin $D$ and cdk4 resulted in reduced phosphorylation of pRb as shown by western blot in arbitrary units $(n=5 /$ group). Representative blots (2/group) are presented below the quantification. ${ }^{*} P<0.05$ compared to WKY, $\# P<0.05$ SHR-Los compared to SHR

We differentiated whether the reduced cell cycle progression resulted in reduced cell count or in attenuated cellular senescence. We measured total renal DNA and observed no

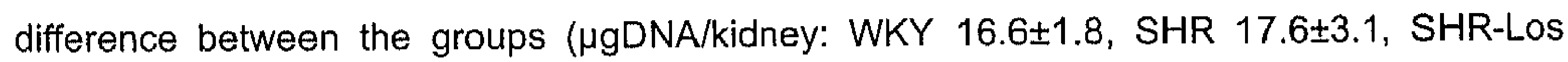
$14.3 \pm 3.2$ ). This suggested that the increased cell cycle progression was largely balanced by increased cell-turnover as SHR groups exhibit no hyperplasia (173). This implicated that the cell cycle length was different between WKY, untreated and treated SHR. We assessed relative telomere length using GPCR (fold) to verify this aspect (193). Telomeres were 
shortest in SHR (0.14 \pm 0.05$)$, twice as long in transiently losartan treated SHR $(0.29 \pm 0.06)$ and 7 times longer in WKY (1.00 \pm 0.07$)$. Similarly, accumulation of oxidative damage as another marker for cellular senescence assessed as renal thiobarbituric acid-reactive substances (TBARS), was significantly reduced in transiently losartan treated SHR at 72 weeks of age $(P<0.05)$ as compared to untreated SHR (Figure 4.3).

Figure 4.3 Urinary excretions of TBARS

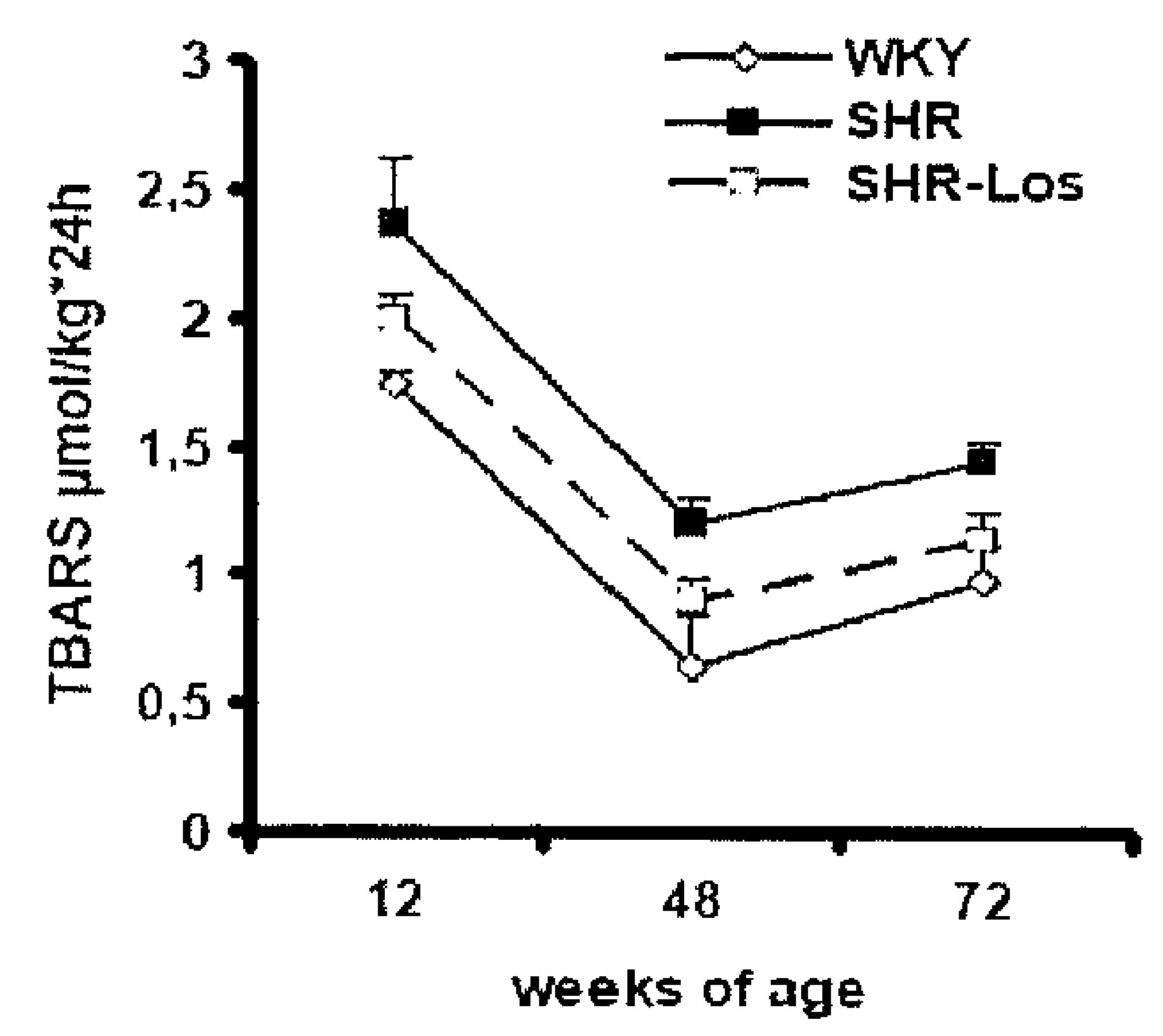

Urinary excretion of TBARS as marker for lipid peroxidation is reduced throughout the observation period in SHR-Los as compared to untreated SHR. \#P<0.05 SHR-Los compared to SHR

Despite similarly elevated blood pressure values in all SHR groups at 18 month of age as previously reported by our group (190), tubular atrophy was reduced in transiently losartan treated SHR as compared to SHR without reaching WKY level (WKY: $0.5 \pm 0.2$, SHR: $1.8 \pm 0.3$, SHR-Los: $1.1 \pm 0.2, P<0.01$ ). Concomitantly we observed a reduction of interstitial collagen deposition in transiently losartan treated SHR (WKY: 4.9 $\pm 1.3 \%$, SHR: $11.3 \pm 2.4 \%$, SHR-Los: $6.4 \pm 1.4 \%$ ) which was confirmed by type IV collagen gene expression ((fold) WKY: $1.0 \pm 0.2$, SHR: $1.7 \pm 0.3$, SHR-Los: 0.5 \pm 0.1 , Figure 4.4A-C).

In contrast to the observations in the renal interstitium, renal vasculature did not demonstrate treatment effects. Neither the cross sectional area (CSA) of afferent arterioles

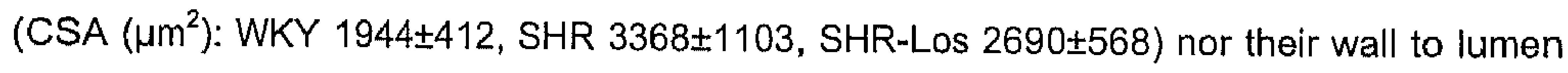

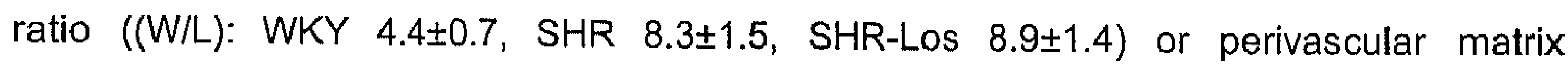

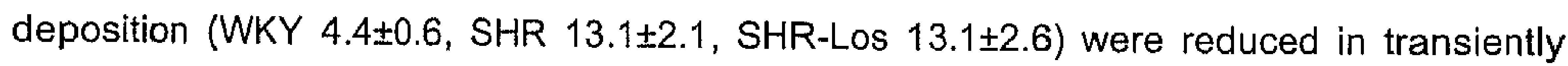
losartan treated as compared to untreated SHR (Figure 3D-F). We examined whether the maintained attenuation of cell proliferation was associated with a reduced ANGII sensitivity. 
Therefore, rats were treated as in the first experiment. Treatment stopped at 8 weeks of age and after a washout period of 4 weeks ANGII

Figure 4.4 Selective renal protection after transient prehypertensive losartan treatment
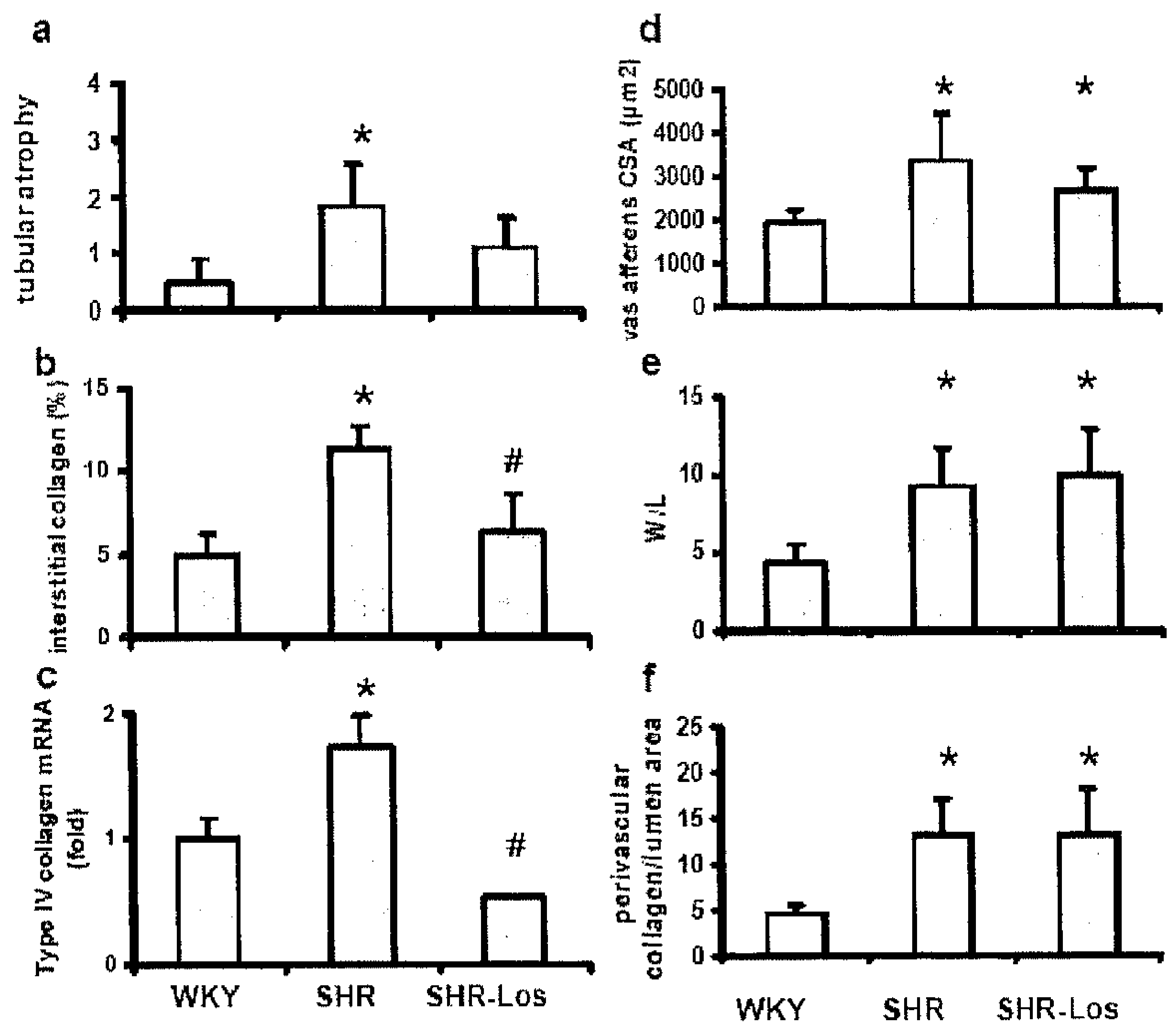

(A) Tubular atrophy, (B) tubulointerstitial collagen deposition and (C) type IV collagen deposition were reduced in transiently treated as compared to untreated SHR. In the renal vasculature no treatment effects were seen on (D) cross sectional area (CSA) and (E) wall to lumen ratio (W/L) of afferent arterioles and $(F)$ their perivascular collagen deposition. ${ }^{*} P<0.05$ compared to WKY, \#P $<0.05$ SHR-Los compared to SHR.

plasma samples demonstrated no differences between the groups, suggesting no differences in basal activity. To investigate ANGIl sensitivity we investigated acute blood pressure responses in the groups by performing ANGII and phenylephrine cumulative dose response curve. Results were evaluated as half maximal blood pressure response (LogEC50). Responses to phenylephrine did not demonstrate group differences. Responses to ANGIl were decreased after transient losartan but not hydralazine treatment (Figure 4.5), suggesting a sustained resetting of angiotensin sensitivity after transient RAAS antagonism in SHR despite similar ANGII plasma levels. 
Figure 4.5 Prolonged reduction of systemic ANGII sensitivity after transient ANGII inhibition

\begin{tabular}{llll}
\hline & SHR & SHR-Los & SHR-Hyd \\
ANGII $(\mathrm{ng} / \mathrm{ml})$ & $55 \pm 19$ & $45 \pm 14$ & $53 \pm 17$ \\
ANGII DRC - $\log$ EC50 & $8.0 \pm 0.2$ & $7.4 \pm 0.1^{*}$ & $8.0 \pm 0.2$ \\
Phenylephrine DRC - $\operatorname{logEC50}$ & $5.9 \pm 0.2$ & $5.8 \pm 0.1$ & $5.9 \pm 0.1$ \\
\hline
\end{tabular}

Plasma ANGII did not differ between the groups four weeks after drug withdrawal, but selectively ANGII sensitivity regarding half maximal blood pressure effects (-LogEC50) was reduced in transiently losartan treated SHR as compared to untreated SHR. Effects of phenylephrine did not differ between the groups. $\# P<0.05$ SHR-Los compared to SHR.

\section{Discussion}

Renal tubular cell proliferation is enhanced in young SHR and reduced by ANGII and TGF- $\beta$ inhibition, but not by general blood pressure reduction. Secondly, transient prehypertensive ANGII antagonism attenuates renal cell proliferation, cell cycle progression and cellular senescence upto advanced age. Transient prehypertensive losartan treatment ameliorates tubulointerstitial damage in SHR, but has no renovascular effects up to advanced age. Finally, transient prehypertensive RAAS antagonism in SHR results in prolonged reduction of ANGIl sensitivity.

Renal tubular cells of young SHR proliferated more than the cells of WKY. These results are in line with previous observations of Hamet et al. who described an increased renal mass index in young SHR (173). Equipotent antihypertensive treatment in young SHR for a four weeks period led to a reduced renal tubular cell proliferation with losartan but not hydralazine. In parallel, plasma TGF- $\beta$ values demonstrated similar characteristics. Therefore we extended previous results demonstrating that ACE-inhibitor but not hydralazine treatment in young SHR reduced proliferation by antagonizing the mitogenic effect of angiotensin, potentially by including the TGF- $\beta$ pathway $(176,194)$.

TGF- $\beta$ is a key mediator of the mitogenic process induced by angiotensin (182). We demonstrated that anti-TGF- $\beta$ treatment reduced renal cell proliferation similarly to losartan without affecting blood pressure. This together with the plasma TGF- $\beta$ values suggest that TGF- $\beta$ is involved in the process of cell proliferation in young SHR. Therefore we conclude 
that ANGII and its mediator TGF- $\beta$, but not blood pressure is the major determinants of cell proliferation in young SHR.

We demonstrated an increased gene expression of the components of the $G 1$ phase in aged SHR as compared to WKY. This reflects the state of advanced cell cycle progression, in which the $\mathrm{G} 1$ phase is of major relevance $(183,195)$. Transient prehypertensive losartan treatment in SHR led to a reduced activation of the G1 phase as compared to untreated SHR. Gene expression of cyclins and cdk's which represent major determinants of the G1 phase are modulated by $A N G \|(185,186)$. Therefore a potential link exists between transient RAS antagonism and sustained reduction of cyclins and cdk's.

A reduced $\mathrm{G} 1$ phase can be caused by an upregulated inhibition of the $\mathrm{G} 1$ phase by proteins as p16, p21 or p27 (195-197). We demonstrated that in SHR transient losartan inhibition did not increase expression of these genes. Therefore the reduced gene expression of cyclins and cdk's and not the inhibitory gene effects on the active G1 product led to the reduced cell cycle progression in SHR after transient prehypertensive losartan treatment.

We compared cellular senescence by means of cell-turnover (telomere length) (193) and intracellular deposition of oxidative damage (TBARS) (198). Aged SHR showed smaller telomeres and increased TBARS, suggesting an advanced cellular senescence in SHR. Therefore we proved the hypothesis of Hamet et al. (173) that SHR show increased cellturnover which led to cellular senescence. Transiently prehypertensive losartan treatment resulted in longer telomeres and reduced TBARS as compared to untreated SHR, suggesting an attenuated cellular senescence by the transient treatment.

To answer the question in how far cellular senescence contributes to a process of accelerated aging which is able to determine cardiovascular target organ damage we separately evaluated areas of high and low cell-turnover. We predicted that areas with high cell-turnover showed more cellular senescence as areas with low cell-turnover. Therefore we chose the kidney as organ of interest as it combines cell types of both characteristics. We defined the renal tubulointerstitium as area of high and the renal vasculature as area of low cell-turnover. Aged SHR demonstrated significantly increased tubulointerstitial atrophy 
and fibrosis as WKY and transiently treated SHR. Thus, cellular senescence in the area of high cell-turnover was associated with the extent of target organ damage. Aged SHR demonstrated significant vascular hypertrophy and perivascular fibrosis. No differences appeared between untreated and transiently treated SHR. Thus, cellular senescence in the area of low cell-turnover did not demonstrate a coherent association with target organ damage. Therefore it seems possible that the target organ damage is modified by accelerated aging. predominantly in areas with high cell-turnover. This does clearly not exclude that in areas of lower cell-turnover cellular senescence has no effects on target organ damage $(176,184)$. This rather suggests that the extent of accelerated aging determines the biological age and cardiovascular target organ damage (199).

Investigating the role of the transient prehypertensive losartan treatment in the deceleration of the aging process we evaluated in how far brief treatment aitered the amount and the sensitivity of ANGII. We demonstrated that four weeks after drug withdrawal no difference in plasma ANGII concentrations exists, but the sensitivity towards ANGII is reduced as compared to untreated SHR. These findings were restricted to the ANGII inhibition. SHR treated with the vasodilator hydralazine did not show these findings, suggesting that in this context ANGII plays the key role. This is additionally reflected by the results that the sensitivity towards phenylephrine was not affected in any of the groups.

These results strengthen the hypothesis of Bergstrom et al. (104) and Nakaya et al. (105) that one reason for the prolonged action of transient prehypertensive treatment is a shift in the ANGII sensitivity. The reduced ANGIl sensitivity could explain that the cell cycle progression remains reduced in these rats as angiotensin is a major activator of the mitogenic process $(185,186)$.

In summary, we demonstrated that the degree of cellular senescence can determine the extent of cardiovascular target organ damage in the SHR. This process is largely ANGII dependent while blood pressure is of minor importance. Transient prehypertensive ANGII inhibition can decelerate the aging process and thereby attenuate target organ damage in areas of high cell-turnover up to advanced age. The prolonged action seems to be related with a sustained shift in ANGII sensitivity which could reduce the mitogenic effect in the SHR. 
CHAPTER FIVE:

Functional and structural postglomerular alterations in the kidney of prehypertensive spontaneously hypertensive rats

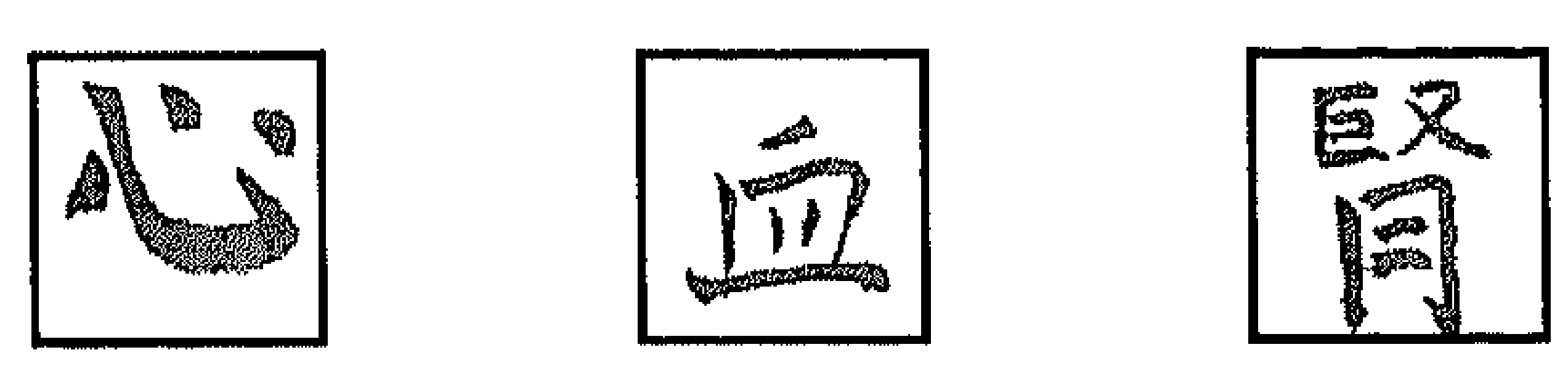

Marcus Baumann, Helma van Essen, JJ Rob Hermans, Jos FM Smits, Harry AJ Struijker- Boudier

Clin Exp Hypertens. 2004; 26(7\&8): 663-672 


\begin{abstract}
The kidney plays a major role in the development of hypertension. Following the BorstGuyton theory of an altered set-point for fluid and electrolyte homeostasis we aim to investigate functional and structural renal parameter during the development of hypertension. Therefore we focus on counter current exchange related factors. We compared 4 and 8 weeks old Wistar Kyoto rats (WKY) and spontaneously hypertensive rats (SHR) concerning basic renal parameters as creatinine and phosphorus clearance and urinary osmolality. Mean arterial pressure (MAP) was measured intra-arterially. Vasa recta were investigated using immunohistochemistry for $\alpha$-smooth-muscle actin (ASMA) and plastification for geometric analyses. Blood pressure was not yet significantly elevated in SHR at 4 weeks but at 8 weeks it was higher in SHR $(116+/-7$ vs. $102+/-4 \mathrm{~mm} \mathrm{Hg} ; p<0.01)$. Kidney weight/body weight ratio was lower in SHR at both ages. In 4 weeks old SHR, phosphorus clearance and urinary osmolality were decreased compared to WKY $(0.02+1-$ 0.01 vs. $0.05+/-0.02\left[\mathrm{ml} / \mathrm{min}^{*} 100 \mathrm{~g}\right.$ BW] $\mathrm{p}<0.03 ; 14.2+/-2.2$ vs. $18.9+/-2.9$ [osmol $/ \mathrm{kg}^{\star} 24 \mathrm{~h}$ urine] $p<0.05$ ) indicating reduced tubular reabsorption. At 8 weeks phosphorus clearance and urinary osmolality were comparable to WKY. a-Actin was found in vasa recta in a 4times higher degree in SHR with a predominant location in the outer medulla. Radii of vasa recta in the outer medulla decreased during development. In plastificated sections vasa recta of SHR revealed sphincter-like pattern. Functional and structural alterations related to the counter current exchanger are already evident in prehypertensive SHR. During development of hypertension both factors get adapted to higher blood pressure level. Sphincter-like structures in vasa recta suggest contractility of pericytes/vascular smooth muscle cells (vSMC). As these were just seen in SHR that might allude to a higher potential to contract. We conclude that differences in postglomerular structure and function may contribute to the development of hypertension in SHR.
\end{abstract}




\section{Introduction}

The kidney plays a key role in the development of hypertension. Borst and Guyton suggested the pressure natriuresis mechanism of the kidney as the most important of all long-term regulators of arterial pressure $(49,200)$. This hypothesis is supported by hereditary forms of hypertension associated with genetic mutations leading to increased sodium reabsorption (81). Even temporary imbalances between salt and water intake and output can alter extracellular fluid volume, arterial pressure and peripheral vascular resistance via autoregulatory mechanisms (52).

Another theory for the kidney's involvement in the development of hypertension is Brenners hypothesis of a low nephron number leading to hyperfiltration by an increase in glomerular pressure to maintain the glomerular filtration rate (GFR) (201). His theory is strengthened by observations of a decreased nephron number in the spontaneously hypertensive rat (SHR) (66) and hypertensive humans (68).

The SHR is the most widely used functional model for the development of essential hypertension. Beside the structural similarities to human condition as above mentioned it has the advantage that in contrast to human situation the prehypertensive phase is well characterized $(202,203)$. During this phase it is known that the extracellular fluid volume is transiently increased (94). According to the Borst-Guyton hypothesis this is an initiating factor for pressure natriuresis. Effectors are the vasa recta as they control medullary blood flow and thereby influence peritubular capillary hydrostatic and colloid osmotic pressure and counter current exchange $(82,204,205)$. The purpose of this study is to investigate the vasa recta function and structure of SHR in early stages of hypertension development. Vasa recta are characterized by capillaries and additional pericytes/vascular smooth muscle cells (VSMC) derived from interstitial cells. These cells were detected by $\alpha$-actin staining (206) and their density and lumen size were evaluated.

\section{Methods}

We compared non-treated WKY and SHR at the age of 4 and 8 weeks ( $n=8$ in each group) concerning their renal function and postglomerular vascular architecture. All experiments were performed according to the institutional guidelines, and were approved by the institutional animal ethics committee. The rats were housed under standard conditions and given rat chow and water ad libitum. The animals were placed in metabolic cages at 4 and 8 weeks of age for $24 \mathrm{~h}$ and were allowed free access to water and food. Urine volume and 
body weight were measured. Furthermore kidney weight was assessed after harvesting and ratios of urine volume per body weight and kidney weight as well as kidney weight per body weight were determined. Collected urine samples from each rat were filtered and centrifuged at $325 \mathrm{~g}$ for $5 \mathrm{~min}$ at $4^{\circ} \mathrm{C}$. The supernatant was collected and immediately frozen and stored at $-20^{\circ} \mathrm{C}$ until use. Blood was collected on the same day and for both urine and plasma, creatinine and phosphorus concentration were determined to compare renal function by estimating creatinine and phosphorus clearances. Urine samples were analysed for osmolality using a standard osmometer (K-7000, Knauer, Germany). Intra-arterial blood pressure was measured under anaesthesia (ketamine/xylazine) as previously described (135). Blood was collected from the $A$. femoralis via the catheter after blood pressure measurement and centrifuged at $3500 \mathrm{~g}$ for $20 \mathrm{~min}$ at $4^{\circ} \mathrm{C}$. The serum was collected and immediately frozen and stored at $-20^{\circ} \mathrm{C}$ until use.

Via the abdominal aorta the left renal artery was perfused with $0.9 \% \mathrm{NaCl}, 0.15 \%$ sodiumnitroprusside and 1000 I.U. heparin under physiological pressure recorded by a pressure transducer (Schwarzer US266). Thereafter, $0.15 \%$ sodiumnitroprusside dissolved in $4 \%$ formalin was perfused in a similar way. Renal vascular tree was infusion fixed using $4 \%$ formaldehyde at a transluminal pressure of $100 \mathrm{~mm} \mathrm{Hg}$. Right kidneys were harvested under aseptic conditions for histology. Immunohistochemistry was performed using antibodies directed against -smooth muscle actin (ASMA, DAKO, U7033) according to protocols supplied by the manufacturer. Quantification was performed by defining anatomical areas of interest, counting the total number of nuclei and creating a ratio of positive stained cells. As area of interest we defined the area of thin descending and ascending tubules including vasa recta and venules.

Plastification of the left renal arteriolar tree was performed by using an epoxy resin. A red metachrylate colour (0.05 $\mathrm{g} / \mathrm{ml}$, Riedel de Haen, Darmstadt, Germany) was added to the epoxy resin (Biodur, Heidelberg, Germany). After intravascular fixation the epoxy resin was installed in vivo until colored epoxy resin entered the $V$. renalis. After several washing steps with $0.9 \% \mathrm{NaCl}$, kidneys were dehydrated in acetone at $-20^{\circ} \mathrm{C}$. In a second plastification step the tissue was plastified under vacuum conditions (Biodur, Heidelberg, Germany) for 15 min. A controlled polymerisation was performed in an oven by stepwise increase of temperature up to $50^{\circ} \mathrm{C}$ in 5-7 days depending on the amount of hardener. Plastified organs were cut with a diamond saw (Leica SP1600, Germany) in 500 $\mu \mathrm{m}$ slices and diameters of vasa recta were analysed. 
Data are reported as mean \pm SD. Comparison between WKY and SHR were made with paired t-tests. Differences were considered significant when $p<0.05$.

Table 5.1 Comparison 4 and 8 weeks old WKY and SHR

\begin{tabular}{lcccc}
\hline & \multicolumn{2}{c}{ Week 4 } & \multicolumn{2}{c}{ Week 8 } \\
MAP [mm Hg] & $73 \pm 9$ & $65 \pm 8$ & $116 \pm 7^{* *}$ & $102 \pm 4$ \\
BW [g] & $105 \pm 15$ & $125 \pm 16$ & $234 \pm 10$ & $243 \pm 16$ \\
KW [mg] & $670 \pm 120$ & $830 \pm 90$ & $1070 \pm 100$ & $1340 \pm 170$ \\
KW [mg]/BW [g] & $5.9 \pm 0.6^{*}$ & $6.7 \pm 0.8$ & $4.5 \pm 0.4^{*}$ & $5.5 \pm-0.8$ \\
\hline
\end{tabular}

MAP: mean arterial pressure, BW: body weight, KW: kidney weight; ${ }^{\star} p<0,05,{ }^{\star *} p<0.01$ vs. age-matched WKY

\section{Results}

Intra-arterial blood pressure measurements demonstrated higher values in SHR at 4 and 8 weeks. However, significant differences versus WKY appeared only at week $8(p<0.01$, Table 5.1). Body weight (BW) showed no significant difference whereas the kidney weight $(\mathrm{KW})$ was decreased in SHR at both ages. The ratio of kidney weight/body weight (KW/BW) was significantly different at week 4 and 8 ( $p<0.05$; Table 5.1). Renal functional parameters are summarized in table 5.2. Total urine volumes as well as Urine/BW ratio were similar at 4 and 8 weeks. However, Urine/KW was higher at $4(p<0.05)$ and 8 weeks $(p<0.05)$ in SHR indicating an increased urine production related to the kidney size.

Table 5.2 Renal functional parameters and ratios in 4 and 8 weeks old WKY and SHR

\begin{tabular}{|c|c|c|c|c|c|c|}
\hline & $\begin{array}{l}\text { Urine } \\
(\mathrm{m} / 24 \mathrm{~h})\end{array}$ & $\begin{array}{l}\text { Urine/KW } \\
(\mathrm{m} 1 / 24 \mathrm{~h}) / \mathrm{mgKW}\end{array}$ & $\begin{array}{c}\text { Creatinine } \\
\text { Clearance } \\
(\mathrm{m} / / \mathrm{min})\end{array}$ & $\begin{array}{c}\text { Phosphorus } \\
\text { Clearance } \\
\text { (m//min) }\end{array}$ & $\begin{array}{c}\text { Total Urine } \\
\text { osmolality } \\
\text { (osmol } / \mathrm{kg}^{*} 24 \mathrm{~h} \\
\text { urine) }\end{array}$ & $\begin{array}{c}\text { Urine } \\
\text { osmolality } / \mathrm{KW} \\
\left.\text { (osmol/ml }{ }^{*} \mathrm{mg} \mathrm{KW}\right)\end{array}$ \\
\hline $\begin{array}{l}\text { SHR } \\
4 \text { wks }\end{array}$ & $7.0 \pm 0.8$ & $1.1 \pm 0.1^{*}$ & $1.2 \pm 0.4$ & $0.02 \pm 0.01^{*}$ & $14.2 \pm 2.2^{*}$ & $22 \pm 4$ \\
\hline $\begin{array}{l}\text { WKY } \\
4 \text { wks }\end{array}$ & $7.8 \pm 0.8$ & $0.9 \pm 0.1$ & $1.4 \pm 0.3$ & $0.05 \pm 0.02$ & $18.9 \pm 2.9$ & $23 \pm 5$ \\
\hline $\begin{array}{l}\text { SHR } \\
8 \text { wks }\end{array}$ & $10.3 \pm 1.6$ & $1.0 \pm 0.2^{*}$ & $0.8 \pm 0.1$ & $0.04 \pm 0.01$ & $22.4 \pm 2.4$ & $21 \pm 2^{*}$ \\
\hline $\begin{array}{l}\text { WKY } \\
8 \text { wks }\end{array}$ & $11.0 \pm 1.6$ & $0.8 \pm 0.2$ & $0.9 \pm 0.2$ & $0.04 \pm 0.01$ & $23.9 \pm 3.2$ & $17 \pm 2$ \\
\hline
\end{tabular}

$\mathrm{KW}$ : kidney weight, ${ }^{*} p<0.05$ vs. age matched WKY 
Creatinine-clearance revealed no differences between WKY and SHR. The phosphorusclearance was decreased only at week 4 in SHR $(p<0.05$, Table 5.2). Measurement of excreted osmotic equivalence confirmed results of phosphorus-clearance since it showed a decreased urinary osmotic equivalence in 4-week old SHR compared to age matched WKY $(p<0.05$, Table 5.2).

Furthermore, this difference disappeared at 8 weeks. Ratios of osmotic equivalence and body or kidney weight at 4 weeks showed no differences, whereas at 8 weeks osmotic products per KW/BW showed reduced excretion in SHR when compared with WKY. At this particular time point total urinary osmotic equivalence was comparable in WKY and SHR. However, due to smaller SHR kidneys the ratios were significantly altered $(p<0.05$, Table 5.2).

Figure 5.1 Visualisation of renal medullary pericytes using alpha-actin immunohistochemistry

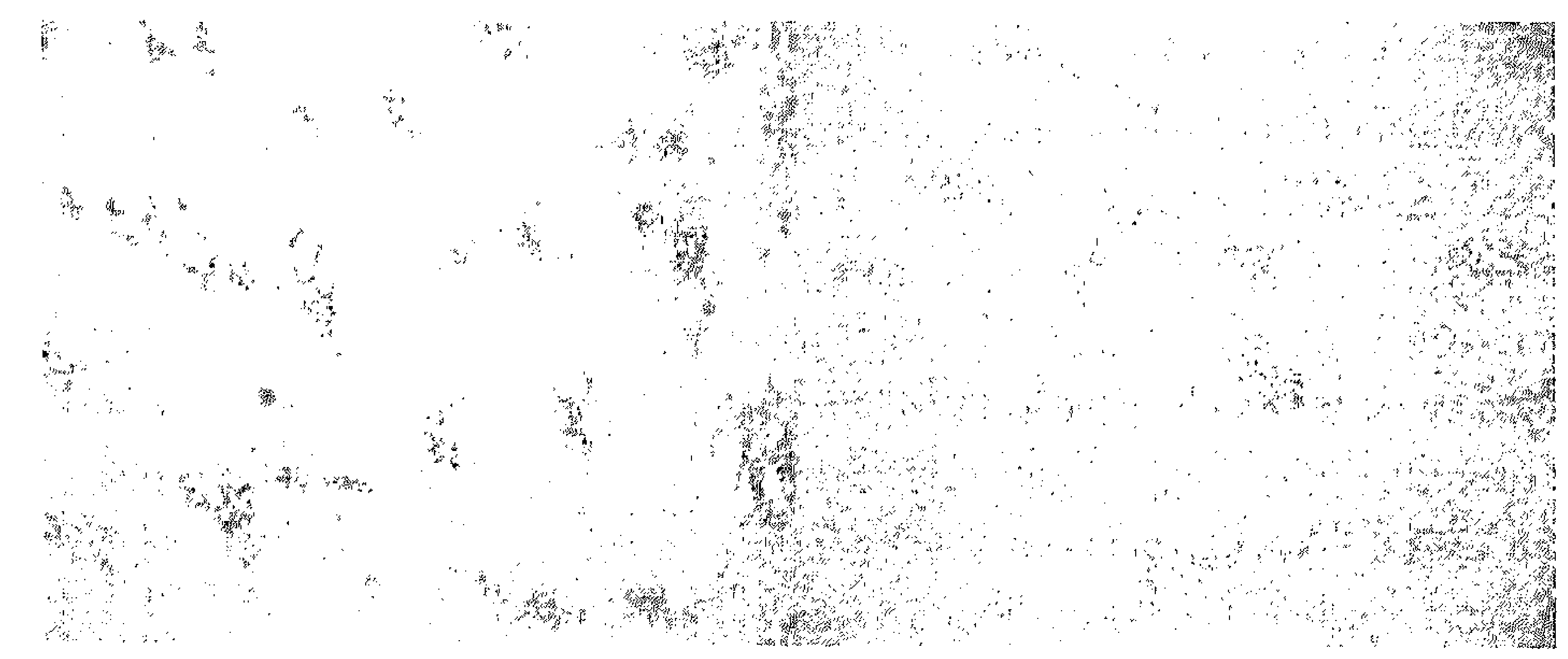

4 weeks old SHR (left) and WKY (right); area of thin ascending and descending tubules; analysis of vasa recta radius revealed comparable values at week 4 in the outer medulla. In the inner medulla vascular radius was significantly smaller in SHR. At 8 weeks results had changed. The radius in the outer medulla of SHR was smaller compared to WKY, whereas no difference was observed in the inner medulla (Figure 5.2). 10X magnification

At 4 weeks $\alpha$-actin staining was more pronounced in SHR compared to WKY in areas of thin descending and ascending tubules at the outer medulla (Figure 5.1). Nuclei of positive cells showed typical corkscrew shape. A counting of a-actin positive cells were performed and the ratio of positive cells and total nuclei number in areas defined as descending and ascending tubules was calculated. This demonstrated a four times higher amount of a-actin positive cells in SHR ( $p<0.05$, Figure 5.2) whereas the total number of cells per area was constant. The difference remained at 8 weeks ( $p<0.05$, Figure 5.2). 
a-Actin positive vasa recta were further differentiated according to their location in outer and inner medulla. This revealed a three times higher concentration of $\alpha$-actin positive cells in the outer medulla of SHR compared to their inner medulla. Differences in WKY were minor (Figure $5.2 \& 5.3$ ). As the question of contractility of a-actin positive cells is still not answered we used the plastification method to investigate the vasa recta for possible sphincter-like structures. These structures were only found in SHR (Figure 5.4).

Figure 5.2 Radius of vasa recta in outer (left panel) and inner medulla (right panel)
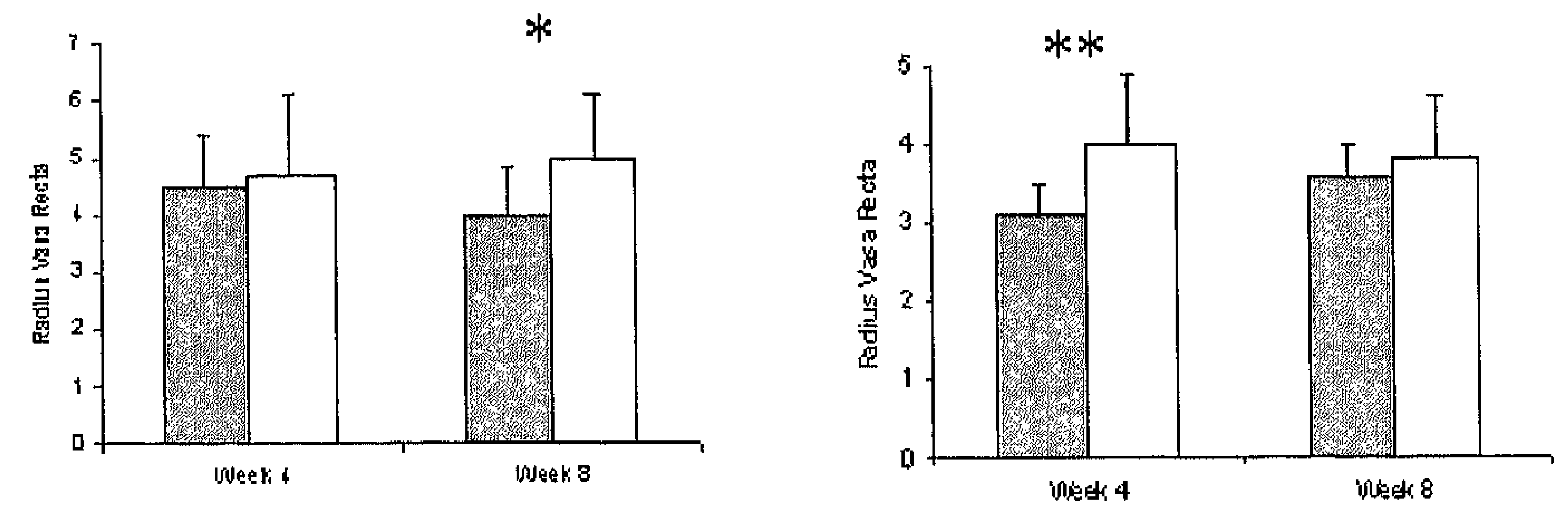

Radii of ASMA positive vessels nuclei in areas of thin descending and ascending tubular cells in 4 and 8 week old SHR and WKY differentiated for outer (left panel) and inner medulla (right panel); $\bullet$ SHR, oWKY; ${ }^{*} P<0.05$ vs $W K Y,{ }^{\star *} P<0.01$ vs WKY

\section{Discussion}

Based on the Borst-Guyton concept the kidney is the key organ for the development of hypertension by altering the set-point of fluid and electrolyte handling (49). Therefore we focused on renal functional parameters to obtain insight in the counter current mechanism and the vascular architecture of the vasa recta as one key regulator of the counter current mechanism. We found smaller kidneys in SHR which can be explained by a lower nephron number (66). Furthermore, we determined a reduced KW/BW at 4 and 8 weeks in SHR. The question arises how lower nephron number and reduced KW/BW might interact with the development of hypertension as they are already evident immediately after birth (66), whereas blood pressure rise starts only several weeks later. Hypertensive humans also show a decreased nephron number (68). If we extrapolate that this is already evident after birth, the time course until development of hypertension lasts several decades. Brenners hyperfiltration hypothesis is often mentioned in this respect (201). As the amount of primary urine is dependent on the pressure difference between pre- and postglomerular vasculature, 
one explanation might be an alteration of the structure and function of the renal preglomerular vasculature. We recently obtained evidence that a decreased nephron number can lead to rising preglomerular resistance and therefore could support the Brenner hypothesis (Baumann et al. abstract Hypertension Vol. 40 No.4 Oct 2002 p591 PG13). Another explanation might be the postglomerular area. The primary urine has to be produced by less glomeruli which means a rise of workload for the whole nephron as reabsorption alters. However, the role of the counter current exchanger during the development of hypertension is still unknown.

Figure 5.3 a-Actin posisitve vasa recta

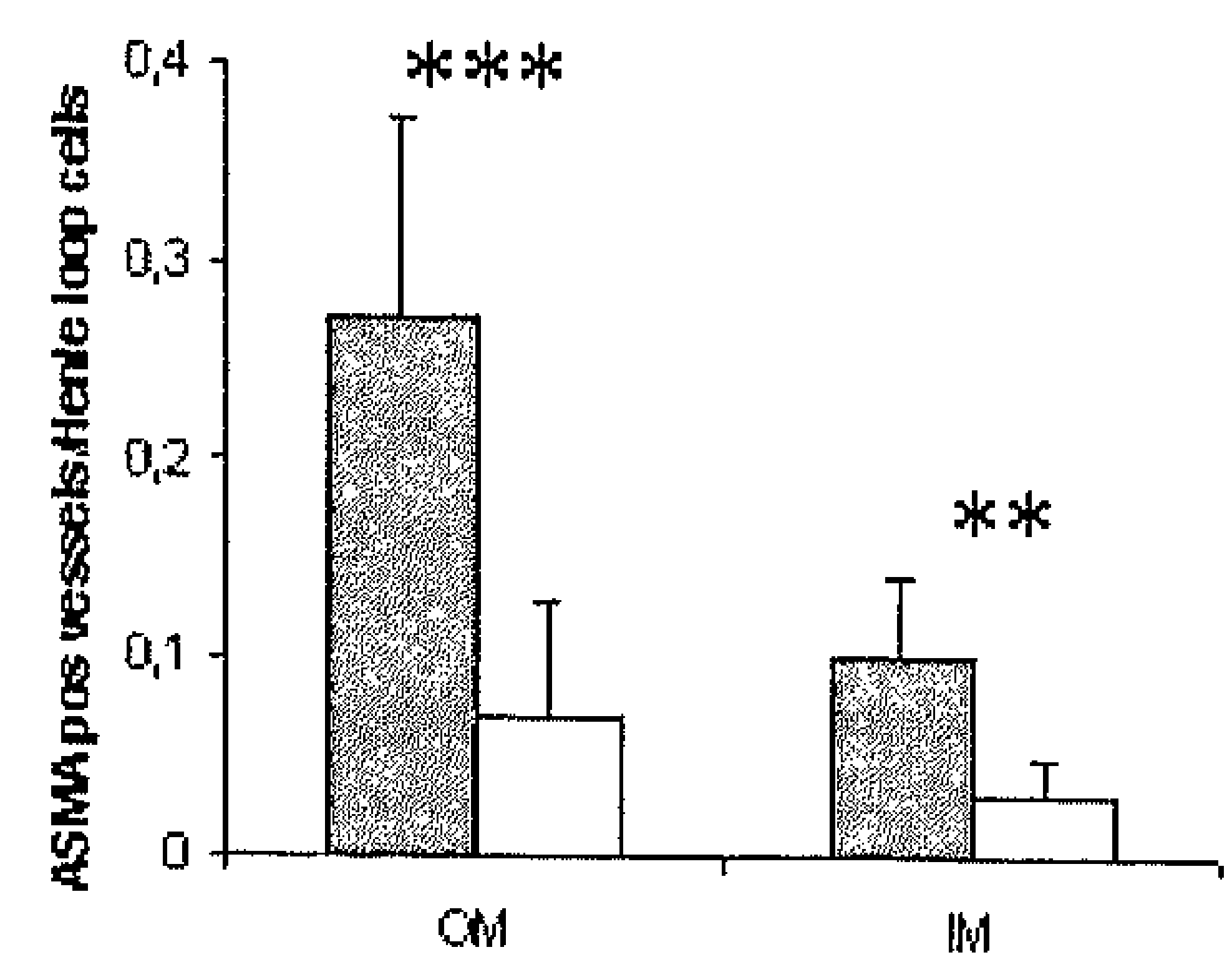
a-Actin posisitve vessels in the outer $(\mathrm{OM})$ and inner $(\mathrm{IM})$ medulla of young SHR $(\bullet)$ and WKY $(0)$;
${ }_{\star \star *} P<0.01$ vs WKY, ${ }^{\star * *} P<0.001$ vs WKY

Reabsorption of phosphorus appears to be increased in SHR at week 4. As this is an unspecific marker of proximal tubular reabsorption, this would point to increased electrolyte retention. In parallel, urinary excretion of osmotic products is decreased, also pointing to decreased excretion and increased reabsorption by SHR kidneys. The latter is more probable given the fact that GFR is similar in SHR and WKY. Hence, at 4 weeks SHR appear to have a more extensive renal tubular reabsorption than WKY, but this is not longer observed at 8 weeks. This corresponds to the observations of Mullins who described an increased extracellular fluid volume during this time period, disappearing at later stage (94, 207). Furthermore, urinary excretion of electrolytes per KW is increasing in SHR at 8 weeks. This may point to decreased tubular reabsorption in SHR to keep extracellular fluid volume stable. As the GFR showed no sign of hyperfiltration whereas both phosphorus and total osmotic equivalence were deranged, we suspected the counter current exchanger enrolled in the increased water and salt retention (49).

80 
Figure 5.4 Plastified vas rectum

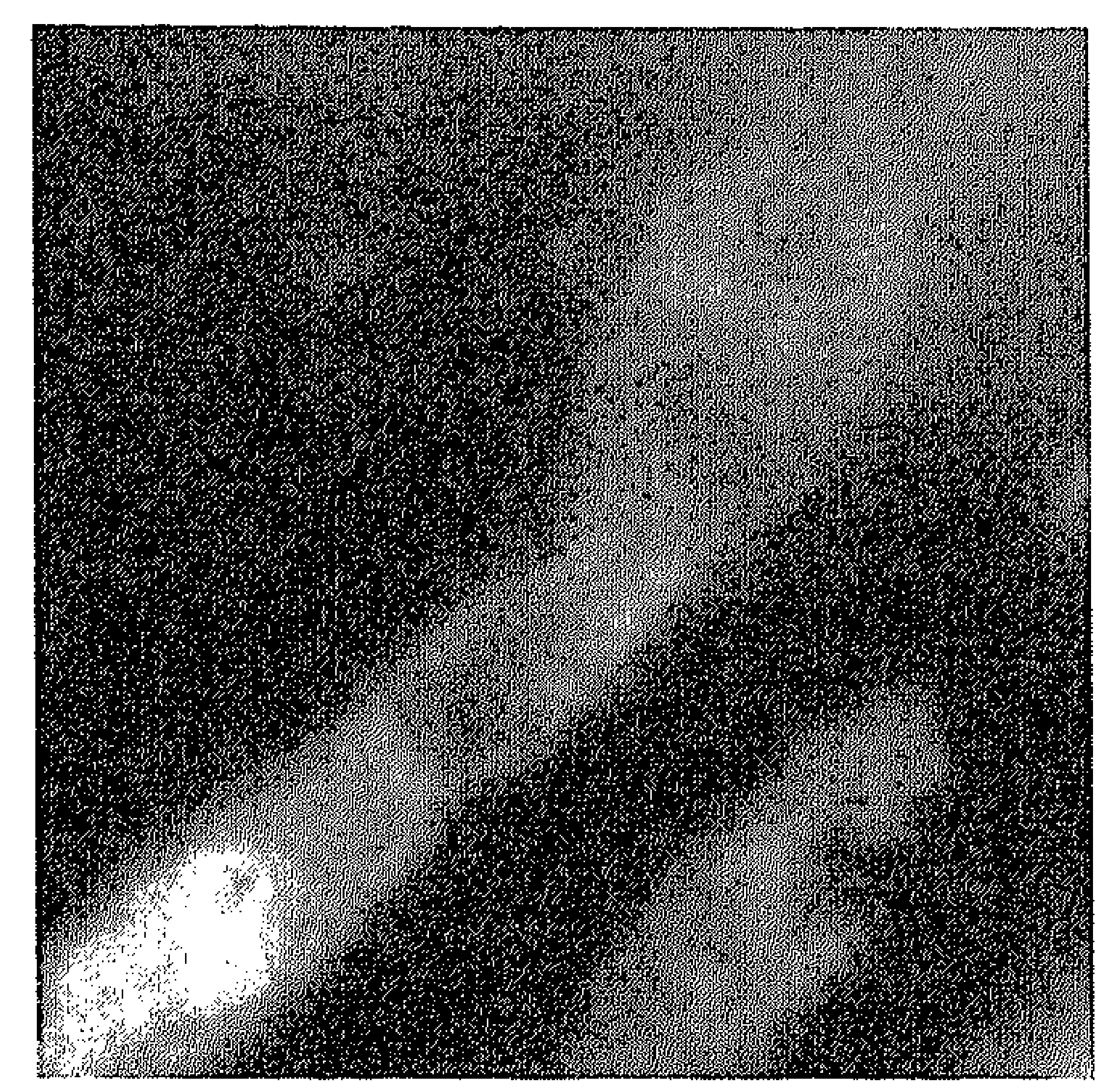

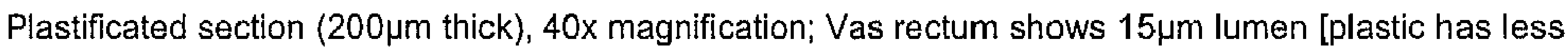
contraction than paraffin], sphincter $(10 \mu \mathrm{m})$ and post sphincter dilation $(26 \mu \mathrm{m})$.

Specifically the vasa recta are of interest as they are needed to build up the osmotic gap to deprive fluid and electrolytes from the primary urine (208). It is known that pericytes/vSMC are present at the site of vasa recta (206). In contrast to tubular or endothelial cells they contain $\alpha$-actin and are thought to be contractile although there is no definite proof. Anatomically they are situated in areas of thin de- and ascending tubules. We found a higher amount of $\alpha$-actin positive cells in SHR at both ages. Maric et al. described that renomedullary interstitial cells are already present at the late embryonic stage and are able to reposition the tubules (209). This implies that prehypertensively the amount of cells may already be higher. The recruitment of $a$-actin positive pericytes/VSMC can be regulated via de novo formation and migration from a preexisting pool (210). As the vasa recta are disconnected from other pericytes/VSMC by the capillary bed of the glomeruli, they might derive from preexisting interstitial cells. The main location of $\alpha$-actin positive cells in the outer medulla may also explain the early effect on the proximal tubuli that we observed.

The contractile elements of pericytes/vSMC can influence the medullary blood flow. Angiotensin-II is able to induce contraction of these cells (211). The a-actin positive cells can be enrolled by altering local resistance, flow or pulsatile pressure. If $\alpha$-actin positive cells correspond to pericyte/vSMC as we hypothesize we have to adhere that till now no proof of 
contractility of this cell type had been established (212). Our observation of sphincter-like structures, which we only found in SHR, may point to possible contractile action. However, this does not answer questions related to resistance and flow regulation locally.

Tubular reabsorption and osmolality also affect each other. Osmolality regulates the angiotensin-ll binding to renomedullary cells (213). The lumen measurements of vasa recta reveal a significantly smaller lumen in the inner medulla at 4 weeks (Figure 5.2). In contrast to this, the lumen of 8 week old SHR is significantly smaller in the outer medulla whereas it was comparable to WKY at week 4 (Figure 5.2). As there is a differential change in lumen in the outer and in inner medulla from the prehypertensive to hypertensive age, this may be of importance. Edwards et al. have shown that higher medullary blood flow is leading to lower reabsorption and higher urinary osmolality per $\mathrm{KW}$ as we have measured (208). However we cannot further hypothesize about local flow, resistance and pulsatile pressure as we did not determine it.

In summary, we described functional and structural alterations which are evident in prehypertensive SHR kidneys. These findings are related to the counter current exchanger and may be linked to the development of hypertension in SHR. 
CHAPTER SIX:

The renal medullary microcirculation as drug target for prehypertensive treatment in young SHR

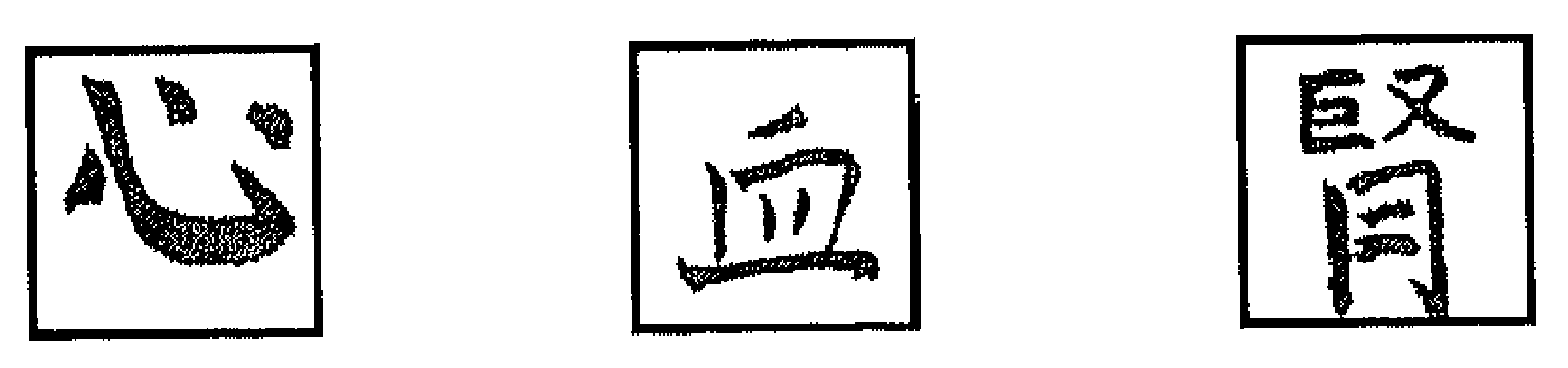

Marcus Baumann, Rob Hermans, Roger Bartholome, Jos F.M. Smits, Harry A.J. Struijker Boudier

in preparation 


\begin{abstract}
Introduction: Renal medullary blood flow (MBF) influences long-term blood pressure control. The medullary microcirculation of SHR is characterized by an increased number of contractile pericytes surrounding the vasa recta. We hypothesize that the number of pericytes modifies blood pressure and that the blood pressure reduction after transient prehypertensive treatment is mediated by a reduction of renal medullary pericytes.

Methods: 4 weeks old SHR were treated for four weeks with losartan (SHR-Los: 20 $\mathrm{mg} / \mathrm{kg} /$ day), hydralazine (SHR-Hyd: $15 \mathrm{mg} / \mathrm{kg} /$ day), losartan in combination with either the pan-caspase inhibitor zVAD (SHR-Los $+1 \mathrm{mg} / \mathrm{kg} /$ day $z V A D$ ) or the Glycogen Synthase Kinase 3beta (GSK) inhibitor valproate (SHR-Los $+10 \mathrm{mg} / \mathrm{kg} /$ day Val). Blood pressure was measured intra-arterially at 8 and 12 weeks of age. Pericyte numbers were determined using $\alpha$-smooth muscle actin immunohistochemistry and Western Blotting. Phophorylated medullary GSK was determined by Western Blotting. Medullary sodium concentration was determined with flame photometry.

Results: Losartan but not hydralazine reduced blood pressure (SHR-Los: $153 \pm 5 \mathrm{mmHg}$, SHR-Hyd: $177 \pm 2 \mathrm{mmHg}$ ) and pericyte number (SHR-Los: $11 \pm 1 \%$, SHR-Hyd: $19 \pm 3 \%$ ) after drug withdrawal and increased medullary sodium concentration and activated GSK as compared to untreated SHR $(184 \pm 3 \mathrm{mmHg}, 22 \pm 2 \%)$. The effects of transient losartan trestment on blood pressure and pericyte number were blocked by adding zVAD $(171 \pm 7$ $\mathrm{mmHg}, 19 \pm 4 \%$ ) or valproate $(174 \pm 3 \mathrm{mmHg}, 20 \pm 4 \%)$.

Conclusion: The number of pericytes modifies blood pressure in SHR. Transient prehypertensive treatment attenuates development of hypertension by initiating apoptosis of renal medullary pericytes in a sodium and GSK dependent manner.
\end{abstract}




\section{Introduction}

Changes in renal medullary blood flow (MBF) influence the long-term control of arterial pressure, although blood flow to this region comprises less than $1 \%$ of the total renal blood flow (72). The renal medullary microcirculation consists of an unique capillary bed. Along the vasa recta there are mesenchymal derived pericytes located which function as the contractile elements (206). The organization of pericytes along the vasa recta allows circumferential contraction. Functional vasomodulation by these pericytes takes place in particular at the level of the outer medulla (214). Vasa recta pericytes contract calcium dependently and are sensitive to angiotensin II (ANG-II) $(214,215)$. We recently described that in young SHR the number of these pericytes is 4-fold increased (216). A greater number of pericytes increases the potential regional vasoconstriction. This can influence local flow and resistance, thereby affecting long-term blood pressure.

Transient prehypertensive treatment with inhibitors of the renin-angiotensin system (RAS) in young SHR leads to a long-lasting fall in blood pressure after drug withdrawal $(103,111)$. The reason for the sustained blood pressure reduction after drug withdrawal remains uncertain, but several authors speculated that the kidney is involved (104, 105, 190). Finding a drug target for transient prehypertensive treatment can improve our understanding of prehypertensive treatment. A reduction of pericytes during the period of transient prehypertensive treatment could reduce resistance for a prolonged time and thereby reduce renal blood pressure. Such a structural modification towards normotensive WKY circumstances could explain the prolonged blood pressure lowering effects of transient prehypertensive treatment after drug withdrawal.

We hypothesize that increased renal medullary pericyte number in SHR is functionally relevant. We furthermore hypothesize that the increased pericyte numbers represent a drug target for transient prehypertensive treatment. We performed transient prehypertensive treatment with an angiotensin II receptor blocker (ARB) in SHR and demonstrate a reduction of renal medullary pericytes. In the following we demonstrate how the ARB reduces renal medullary pericytes and how this process is causally related to the sustained blood pressure reduction after transient prehypertensive treatment. 


\section{Methods}

Male WKY $(n=12)$ and SHR $(n=37)$ were obtained from Charles River (Landgraaf, The Netherlands). The rats were fed a normal sodium diet and had free access to water. All experiments were approved by the animal ethics committee of the Maastricht University and were performed in accordance with institutional guidelines.

WKY and SHR were treated between 4 to 8 weeks of age. WKY and SHR were given saline (as control), losartan (SHR-Los: $20 \mathrm{mg} / \mathrm{kg} / \mathrm{day} ; \mathrm{n}=13$ each group) or hydralazine (SHR-Hyd: $15 \mathrm{mg} / \mathrm{kg} /$ day; $\mathrm{n}=12$ ), losartan in combination with the pan-caspase inhibitor ZVAD (SHRLos/zVAD: $20 \mathrm{mg} / \mathrm{kg} /$ day losartan $+1 \mathrm{mg} / \mathrm{kg} /$ day zVAD; $\mathrm{n}=6$ ) or losartan in combination with the GSK inhibitor valproate (SHR-Los $/$ al: $20 \mathrm{mg} / \mathrm{kg} /$ day (LoS) $+10 \mathrm{mg} / \mathrm{kg} / \mathrm{day}(\mathrm{Val}) ; \mathrm{n}=6$ ). for a period of four weeks using osmotic minipumps (Alzet 2004, Durect, CA, USA) that were implanted subcutaneously under isoflurane anaesthesia $(1.5 \%)$.

At 8 and 12 weeks of age blood pressure was measured in the conscious unrestrained, state through a heparinized indwelling polyethylene catheter that was introduced into the left femoral artery two days before measurement (135). After blood pressure measurement, rats were killed by exsanguinations and kidneys were harvested, transferred into $4 \%$ formalin or dissected into cortical and medullary tissue and immediately frozen at $-80^{\circ} \mathrm{C}$. The formalin fixed tissues were paraffin-embedded to analyze for each animal the lumen diameter of vasa recta surrounded by $a$-smooth muscle actin containing pericytes. Therefore immunohistochemistry for a-smooth muscle actin (ASMA, DAKO, U7033) was performed on $4 \mu \mathrm{m}$ parallel transversally cut slices and vasa recta lumen diameters defined as vasa recta surrounded by contractile pericytes of the outer medulla were determined. At least 20 vasa recta per slice were measured as previously described (216). Briefly, transversally cut medullary vasa recta with a surrounding pericyte were localized with a Zeiss Axioscope (Zeiss, Jena, Germany) and a standard CCD camera (Stemmer, Munich, Germany) using a 40x magnification. Lumen diameter was calculated from the internal circumference assuming a circular cross-section (JAVA 1.21; Jandel Scientific, Corte Madera, CA, U.S.A.). Frozen renal medullary tissue from WKY and SHR ( $n=5 / g r o u p)$ was prepared for Western Blotting (160) Membranes were incubated with rabbit polyclonal antibody against ASMA (50 $\mathrm{kDa}$ ) or goat polyclonal antibody against phosphorylated GSK (44

kDa) (Santa Cruz) diluted 1:200 in washing solution at room temperature for 1 hour. The membranes were then washed, incubated with anti-rabbit or anti-goat horseradish peroxidase-conjugated second antibody 1:2000 for 1 hour at room temperature, and washed 
extensively. Membranes were incubated with Chemiluminescence Blotting Substrate (Boehringer Mannheim), according to the manufacturer's protocol, and exposed to film that was immediately developed. Detection was performed with a Zeiss Axioscope (Zeiss, Jena, Germany and a standard CCD camera (Stemmer, Munich, Germany). Band intensity was measured by computer analysis, using the LeicaQwin program.

All parameters are expressed as means \pm S.E.M. Data obtained after pharmacological intervention were compared using an ANOVA for repeated measures and a post hoc Dunnett's t-test to identify differences between the groups. $P$ values $: 0.05$ were regarded to indicate statistical significance.

\section{Results}

Transient prehypertensive treatments using losartan and hydralazine were performed to investigate the treatment effect on the pericyte number. Blood pressure was initially reduced by losartan and hydralazine. After drug withdrawal of losartan but not hydralazine blood pressure remained significantly lower. We investigated whether the pericyte number was affected by the treatment four weeks after drug withdrawal in 12 weeks old SHR. Immunohistochemistry for ASMA demonstrated a a significantly lower amount of ASMA positive cells on the renal medulla in WKY and SHR-Los. Western blotting of renal medullary tissue for ASMA confirmed these results (Figure 6.1).

To investigate whether the reduction of pericytes was relevant for the blood pressure lowering effect and whether apoptosis is involved in the process we repeated the losartan treatment adding the pan-caspase inhibitor $Z V A D$ during the same period of time. Combined losartan/zVAD treatment during 4-8 weeks of age resulted four weeks after drug withdrawal in an elevated blood pressure level as compared to ARB monotherapy. The pericyte number was significantly higher in the group which was treated with losartan/zVAD as compared to ARB monotherapy (Figure 6.2). 
Figure 6.1 Blood pressure and medullary pericytes

A

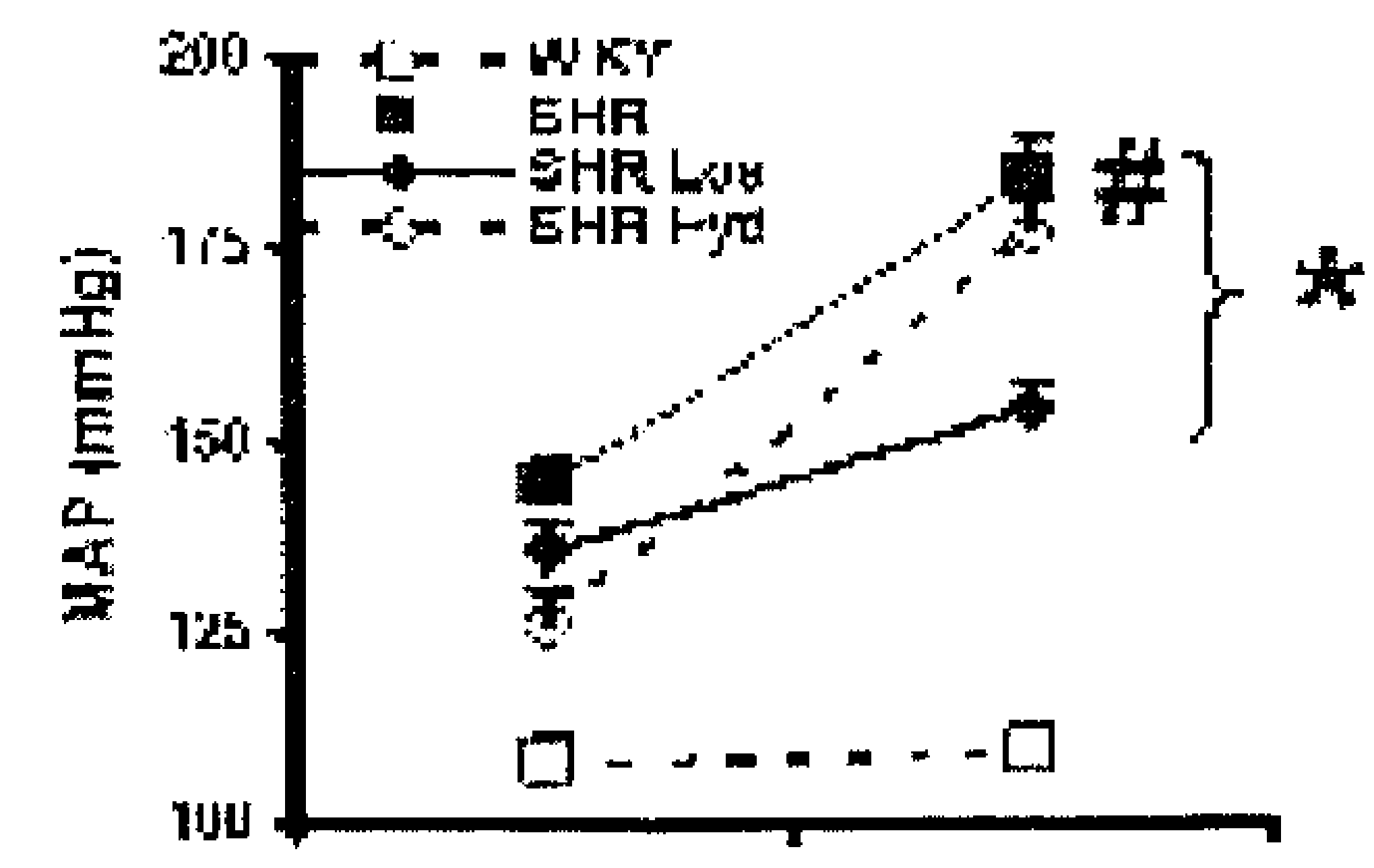

B.

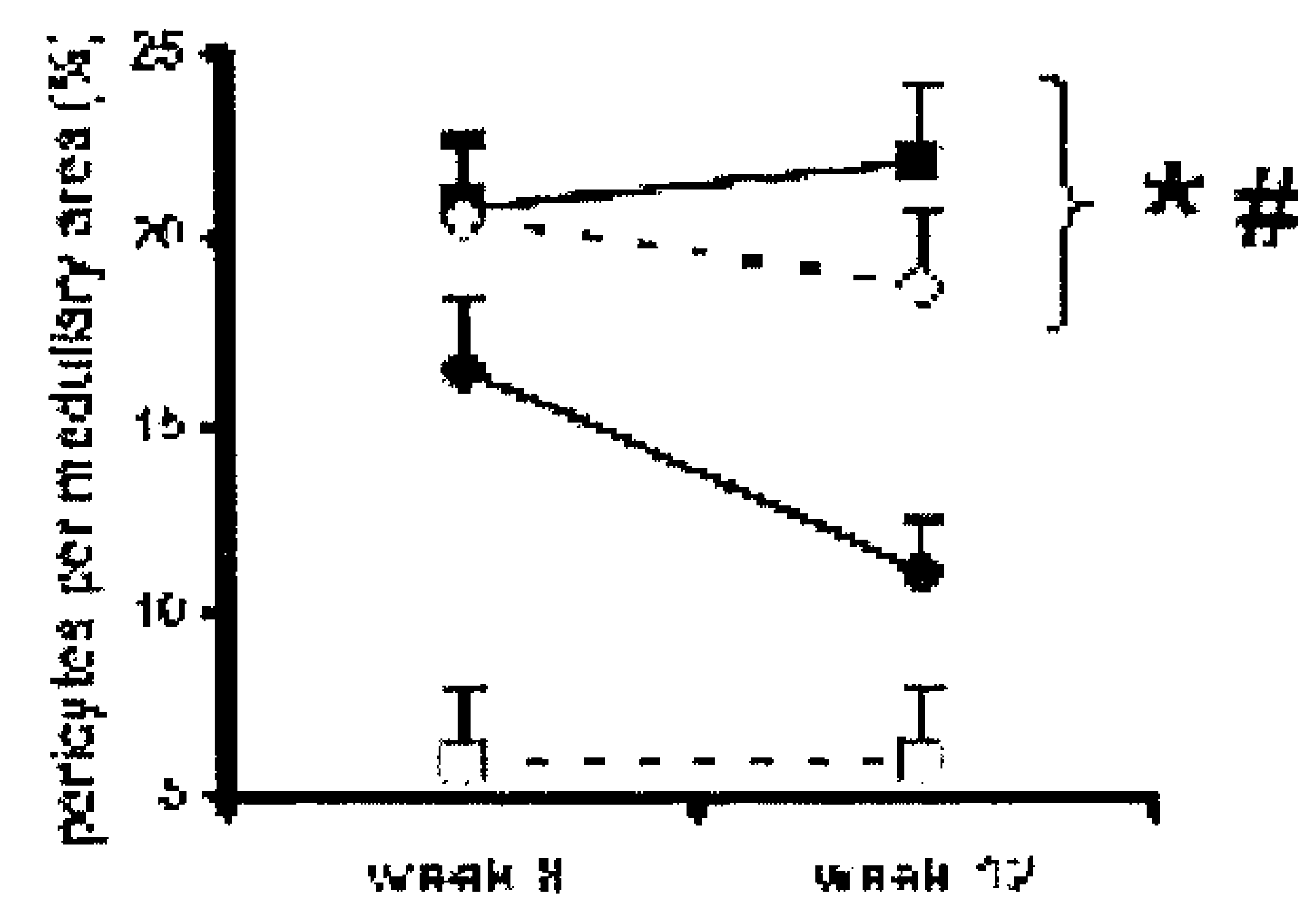

$E$

WUKY

SHR
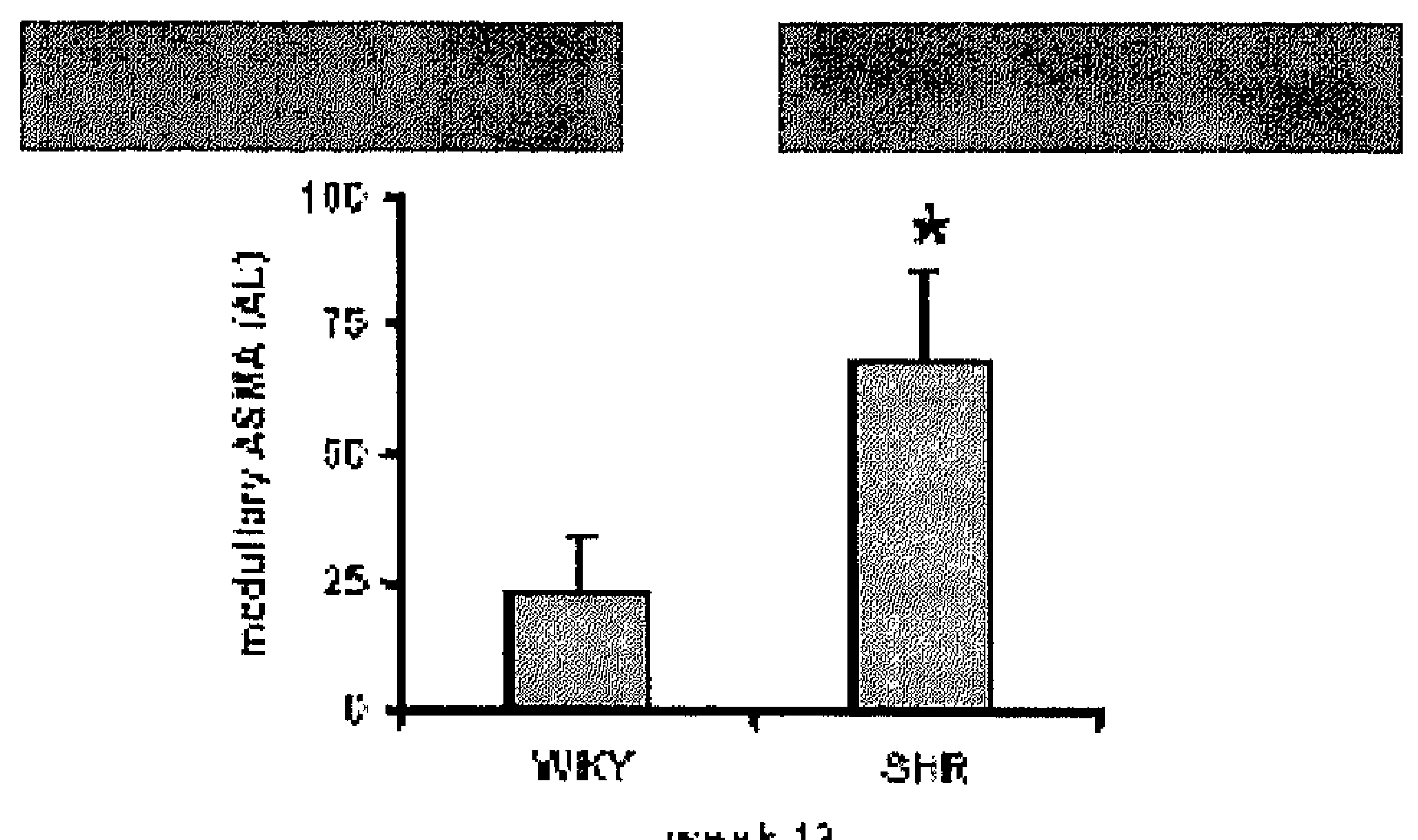

Figure 6.1A demonstrates the blood pressure development at 8 and 12 weeks of age in WKY, SHR, SHRLos and SHR-Hyd. Blood pressure increase is significantly reduced by losartan and hydralazine treatment at 8 weeks of age. After drug withdrawal blood pressure increase is delayed only in previously losartan treated SHR. Figure 6.1B demonstrates that the number of medullary pericytes is reduced by the losartan treatment and reaches WKY level at 12 weeks of age. Figure 6.1C demonstrates the Western Blotting of ASMA at 12 weeks of age. SHR-Los demonstrate significantly less ASMA as untreated SHR. 6.1A and 6.1B: ${ }^{*} P<0.05$ as compared to $W K Y, \# P<0.05$ as compared to $S H R, 1 C^{\star} P<0.05$ as compared to SHRLos 
Figure 6.2 Combined AT1R and caspase-inhibition
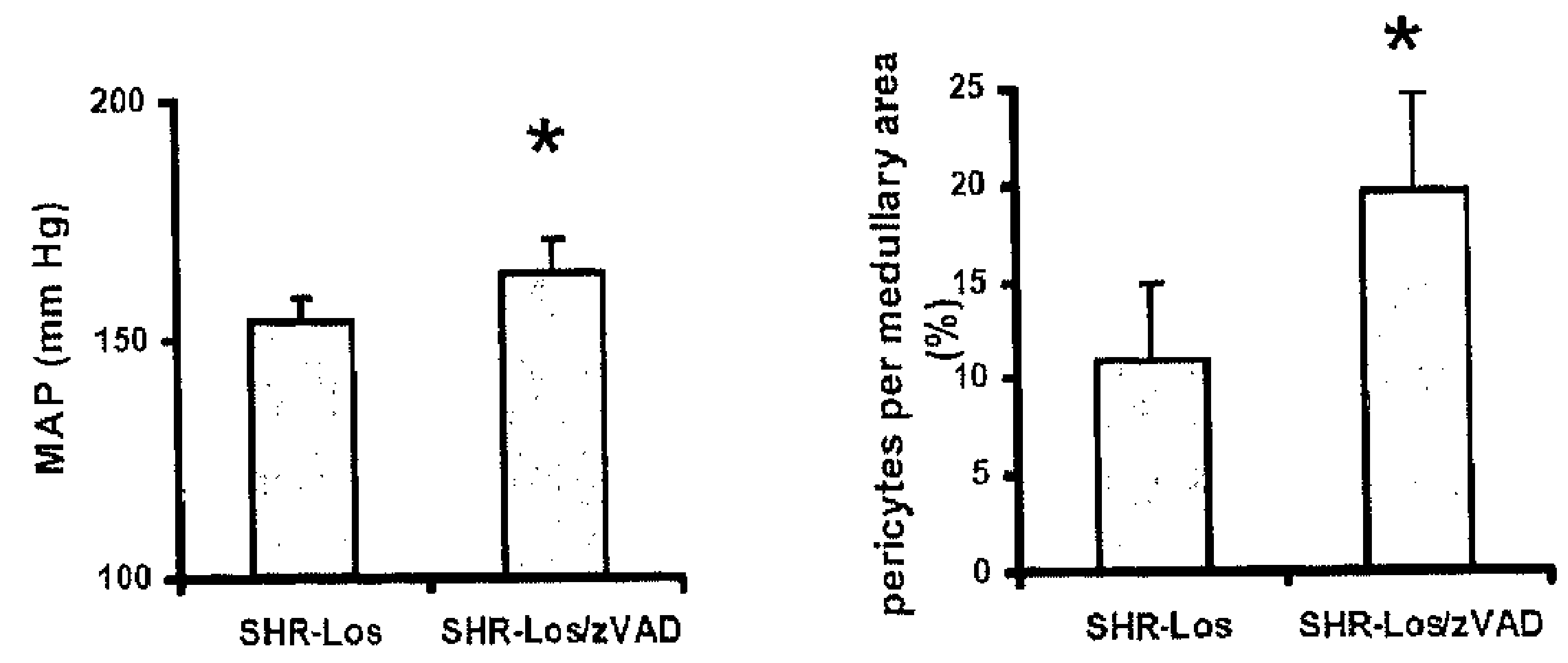

Figure 6.2 demonstrates the small, but significant blood pressure increase after combination therapy of $A R B$ and pan-caspase inhibitor ZVAD. In parallel increases the number of pericytes under this combination as compared to ARB monotherapy. ${ }^{*} P<0.05$

A recent study investigated the role of sodium on apoptosis of pericytes involving a GSK dependent mechanism (217). Therefore, the sodium concentration was measured in the tip of the medulla. Sodium concentration was elevated in WKY and SHR-Los demonstrating similar levels. In contrast, untreated SHR and SHR-Hyd had significantly lower sodium concentration in the renal medulla (Figure 6.3).

Figure 6.3 Medullary sodium content is increased after transient losartan treatment

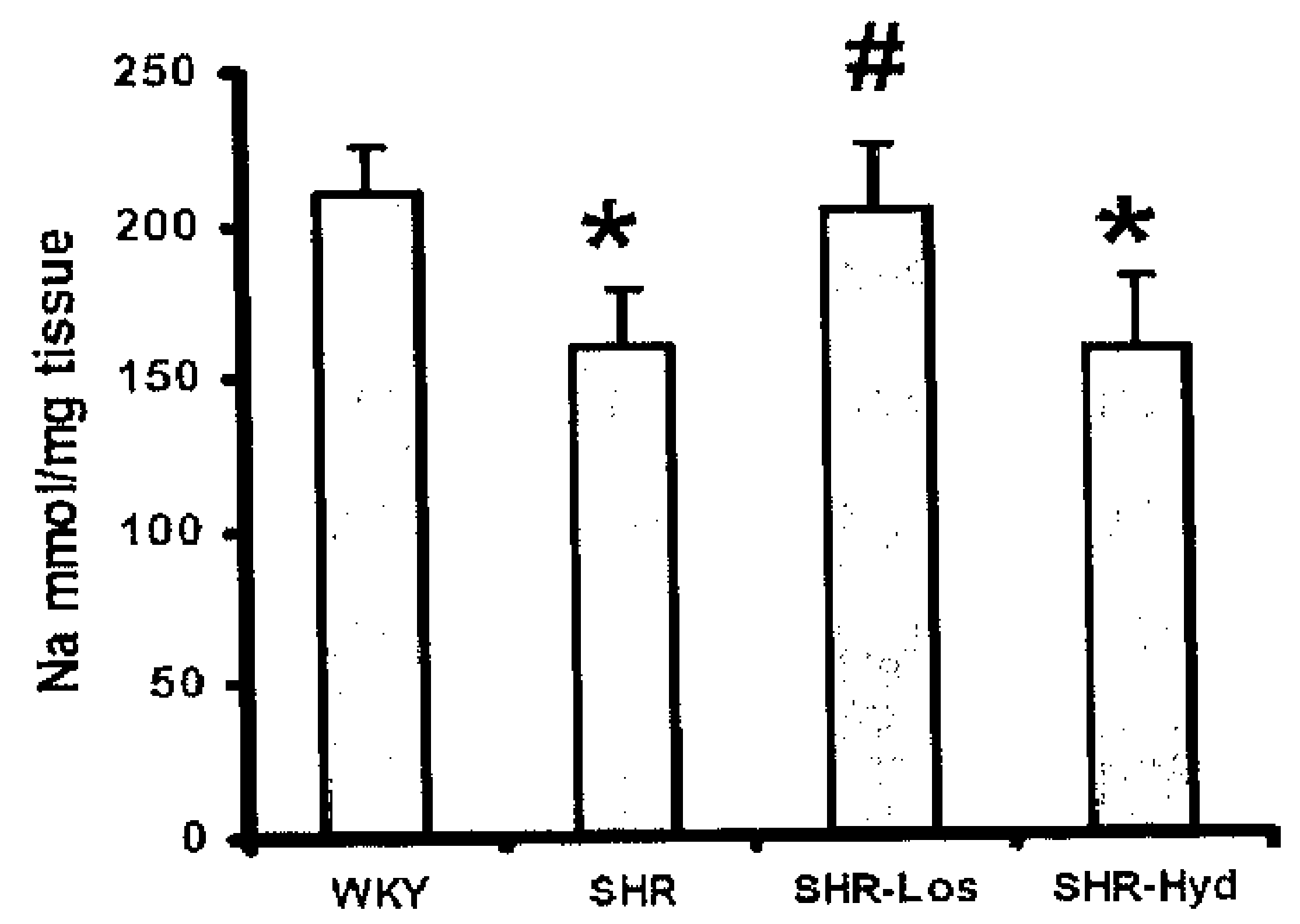

Figure 6.3 demonstrates the sodium content of the tip of the renal medulla in WKY, SHR, SHR-Los and SHR-Hyd at 12 weeks of age. ${ }^{*} P<0.05$ as compared to WKY, \# P<0.05 as compared to SHR.

Phopshorylated renal medullary GSK content was quantified using Western blotting. Phosphorylated GSK signal was stronger in WKY and SHR-Los as compared to SHR and SHR-Hyd (Figure 6.4). 
Figure 6.4 Phosphorylated GSK in the renal medulla
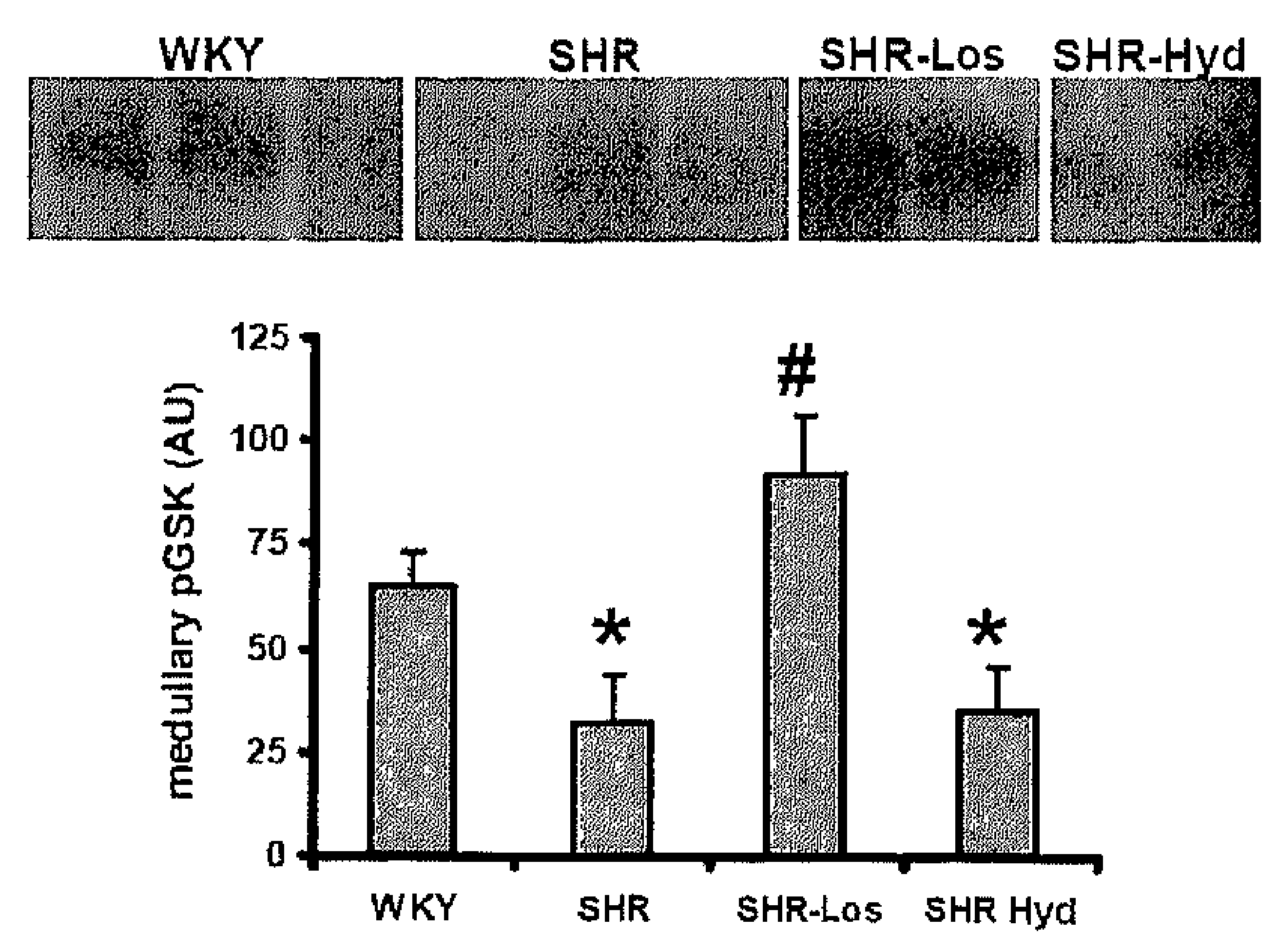

Figure 6.4 demonstrates the amount of phosphorylated GSK in the renal medulla of WKY, SHR, SHR-Los and SHR-Hyd at 12 weeks of age. ${ }^{\star} P<0.05$ as compared to WKY, $\# P<0.05$ as compared to SHR.

The role of GSK on blood pressure and pericyte number was tested in a comparison between transient losartan treatment and combined losartan and GSK inhibition with valproate. Four weeks after drug withdrawal blood pressure was higher in the group which received combination therapy as compared to the losartan monotherapy. The pericyte number was significantly higher after the combination therapy as compared to the losartan monotherapy (Figure 6.5).

Figure 6.5 Combined AT1R and GSK-inhibition
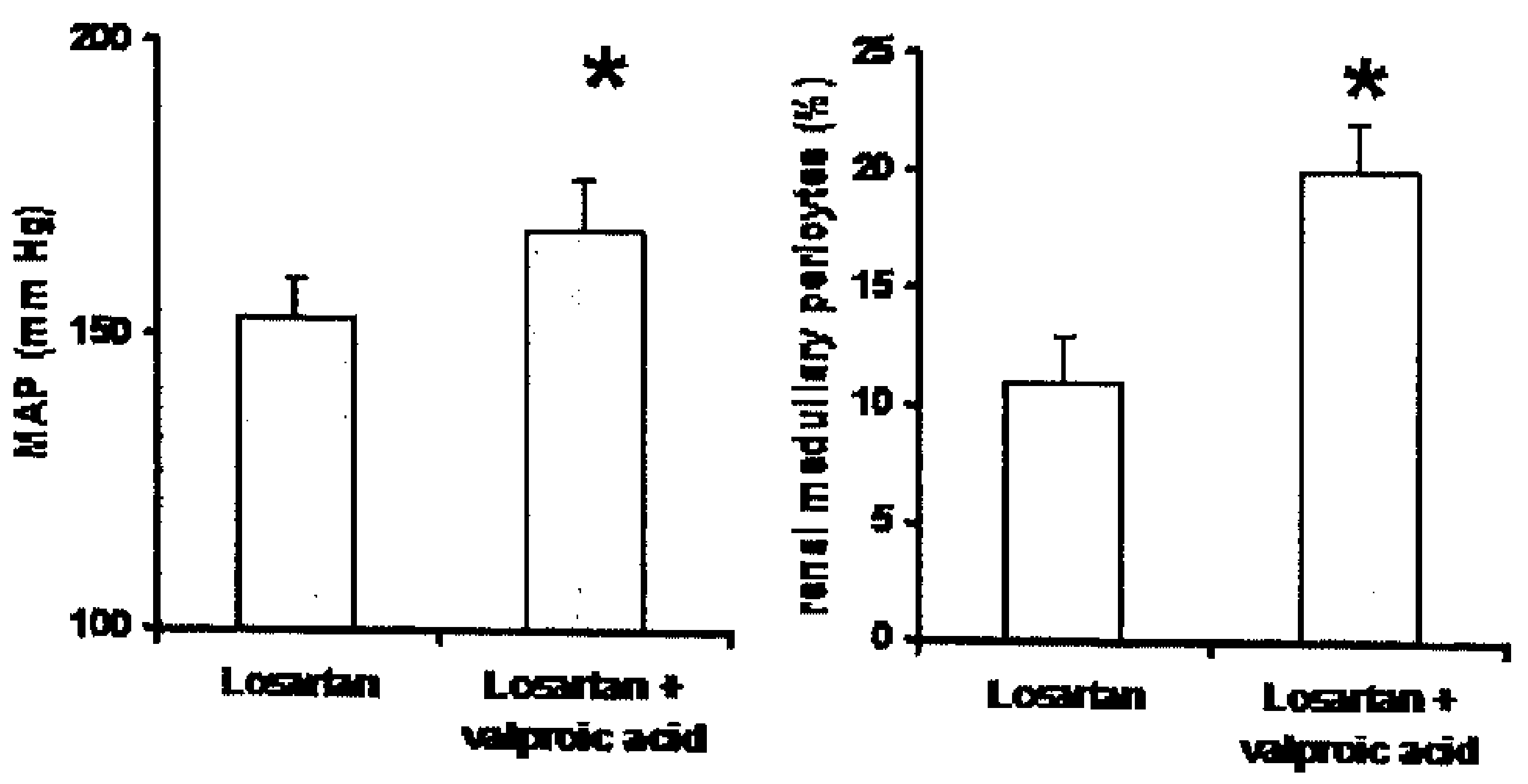

Figure 6.5 demonstrates the small, but significant blood pressure increase after combination therapy of $A R B$ and pan-caspase inhibitor $Z V A D$. In parallel increases the number of pericytes under this combination as compared to ARB monotherapy. ${ }^{*} P<0.05$ 


\section{Discussion}

Transient prehypertensive treatment using an ARB reduces blood pressure and renal medullary pericyte number in young SHR. Both findings are associated with an apoptotic process initiated by hypertonic stress and consecutive GSK activation. ARB in combination with a pharmacological inhibition of the apoptotic process increases blood pressure and renal medullary pericyte number. Similarly, ARB in combination with a pharmacological inhibition of GSK increases blood pressure and renal medullary pericyte number.

We recently demonstrated that the renal medullary pericyte number is 4-times higher in SHR as compared to WKY (216). Transient prehypertensive treatment with an ARB reduces blood pressure and pericyte number in SHR. This suggests that pericytes might be involved in the blood pressure lowering process. The renal medulla has been evidenced to participate in blood pressure homeostasis by modifying the renal medullary blood flow in a RAS associated manner $(73,218)$. As pericytes are contractile $(206)$ and sensitive to angiotensin II (215) they could be involved in this process. In contrast, hydralazine treatment did not reduce the pericyte number and after withdrawal of the treatment blood pressure restored to untreated levels. This additionally points to the RAS as a system involved in the sustained blood pressure lowering and the reduction of pericyte numbers. Thirdly, we can exclude that the initial blood pressure lowering is responsible for the reduction of pericytes as during hydalazine the blood pressure was significantly reduced, but the pericyte number remained unaffected.

This observation suggests that the sustained blood pressure lowering after prehypertensive treatment is associated with the reduction in pericytes. However, it remains uncertain whether the reduction of renal medullary pericytes represents only an epiphenomenon or a relevant mechanism.

To clarify this point we inestigated whether the reduction of pericytes is caused by apoptosis induced by the ARB (219). Therefore we added to the ARB monotherapy a pan-caspase inhibitor (220). The main finding of this experiment was that the combined therapy had higher blood pressure and a higher pericyte number. This suggests that the ARB induced not only the blood pressure reduction, but also induced apoptosis in renal medullary pericytes. The pan-caspase inhibitor is not known to increase blood pressure by itself $(220$, 
221), therefore the hypothesis is strengthened that the number of renal medullary pericytes are causally related to the sustained blood pressure effect.

It remains uncertain whether the apoptotic mechanism is specific for the medulla or a general vascular phenomenon. To elucidate this point we investigated whether a medullary specific aspect induces pericyte apoptosis. An important mechanism to induce apoptosis in the medulla is a hypertonic environment. (222). The renal medulla is a hypertonic surrounding which is related to the counter current mechanism $(208,223)$. This makes the medulla unique in the body (204). By measuring the hypertonic stress in the renal medulla we confirmed earlier observations that the hypertonic stress is higher in WKY as compared to SHR (224). Furthermore transient prehypertensive losartan but not hydralazine treatment increased the renal medullary sodium concentration. This demonstrates that in SHR only the drug which leads to the sustained lower blood pressure has an effect on the hypertonic state of the renal medulla. Hypertonic stress induces apoptosis by an activation of GSK in pericytes in vitro (217). In the Western blot our results confimed this idea for the renal medulla. GSK activaton paralleled the rise in renal medullary sodium concentration. This suggests that transient prehypertensive treatment induces hypertonic stress which activates GSK mediated apoptosis of renal medullary pericytes. To test this concept a combined treated with an ARB and the unspecific GSK-inhibitor valproate was perfomed. The effect of valproate as a GSK inhibitor has been described by several authors $(217,225)$. An effect on blood pressure was yet not described. The experiment proved that the reduction of renal medullary pericytes by an ARB is attenuated by a GSK-inhibitor and that the blood pressure is higher as compared to the montotherpay with an ARB.

We conclude at this point that transient prehypertensive treatment has direct renal medullary effects leading to sustained lower blood pressure values after drug withdrawal. Based on our results we postulate the following mechanism: Transient prehypertensive treatment with an ARB causes in young SHR an increase in renal medullary osmolality. This hypertonic stress activates GSK mediated apoptosis of pericytes. Thereby the number of renal medullary pericytes is reduced towards WKY level. This reduction causes a sustained alteration of renal medullary flow which attenuates blood pressure development in SHR. Whether additional pathways are included in this the blood pressure lowering cannot be excluded. 
These data further suggest that reduction of renal medullary pericytes can cause blood pressure lowering. Therefore pericytes offer a potential drug target for antihypertensive treatment. In this respect it is possible that the blood pressure lowering effects of renal medullary ACE-inhibition are at least partially mediated by pericyte reduction (73).

In summary we describe that the sustained blood pressure lowering after transient prehypertensive treatment with an ARB is caused by a reduction of renal medullary pericytes. The reduction results from medullary hypertonicty which induces GSK-mediated apoptosis of pericytes in the renal medulla of SHR. 


\section{CHAPTER SEVEN:}

Potential individual prediction of the progression form prehypertension to new-onset hypertension

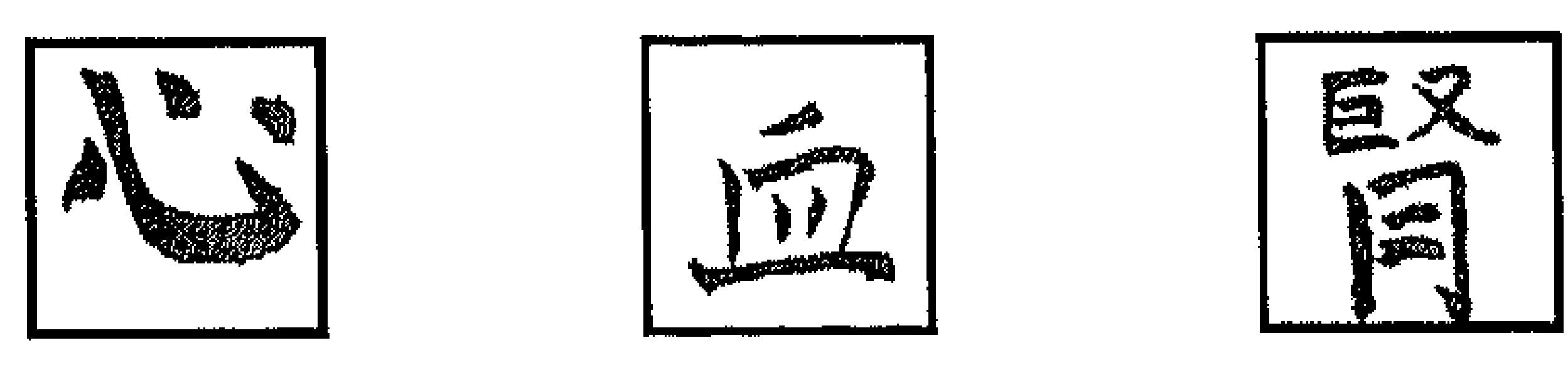

Marcus Baumann, Tatiana Kouznetsova, Roger Bartholome, Jos FM Smits, Harry Struijker Boudier, Jan Staessen

In preparation 
Abstract

Background: Prehypertensive individuals demonstrate an increased rate to progress to hypertension. Individual prediction at which time point hypertension establishes is yet impossible, but would potentially allow earlier antihypertensive therapy or even prevention. We aim to find potential predictors which can characterize the progression from prehypertension to hypertension based on characteristics seen in animal models.

Methods: In a nested case-control study covering a period of 4.5 years we built 4 groups according to JNC-VII: prehypertensive individuals remaining prehypertensive (PHT $\rightarrow$ PHT, $n=8$ ), prehypertensives developing hypertension during the observation period (PHT $\rightarrow H T$, $n=13$ ), individuals remaining hypertensive $(H T, n=12)$ or normotensive (NT, $n=18$ ). We investigated urinary nitric oxide metabolites (NOx) as marker for NO bioavailability. 24-hour creatinine-clearance and sodium excretion were determined as renal marker at start and end of the study period.

Results: Urinary NOx were decreased in PHT $\rightarrow H T(124 \pm 23 \mu \mathrm{g} / \mathrm{ml})$ and HT $(114 \pm 23 \mu \mathrm{g} / \mathrm{ml})$ as compared to NT $(216 \pm 21 \mu \mathrm{g} / \mathrm{ml})$. PHT $\rightarrow$ PHT demonstrated normotensive NOx values $(192 \pm 23 \mu \mathrm{g} / \mathrm{ml})$. Sodium excretion was decreased in PHT $\rightarrow H T(138 \pm 20 \mathrm{mmol} /$ day $)$ as compared to other groups (NT: $196 \pm 18 \mathrm{mmol} /$ day, PHT $\rightarrow$ PHT: $194 \pm 25 \mathrm{mmol} /$ day, HT: $211 \pm 21 \mathrm{mmol} /$ day) and was normalized after 4.5 years. This resulted in a significant increase of sodium excretion in $\mathrm{PHT} \rightarrow \mathrm{HT}(54 \pm 15 \%)$ as compared to other groups (NT: $9 \pm 9 \%$, $\mathrm{PHT} \rightarrow \mathrm{PHT}: 15 \pm 8 \%, \mathrm{HT}: 10 \pm 11 \%)$. 24-hour creatinine-clearance was reduced in both prehypertensive groups to the same extent as in HT.

Conclusion: In prehypertensive individuals the development of hypertension is associated with decreased urinary NOx and transiently decreased sodium excretion. Therefore, the combination of these factors potentially predicts the individual onset of hypertension during a period of 4.5 years. 


\section{Introduction}

Observational studies in adults demonstrated that the risk of cardiovascular disease increases progressively from levels as low as $115 / 75 \mathrm{~mm} \mathrm{Hg}$ upward with a doubling of the incidence of both coronary heart disease and stroke for every $20 / 10 \mu \mathrm{mm} \mathrm{Hg}$ increment of blood pressure (226). The rate of progression towards hypertension from other than optimal blood pressure levels is enhanced (10). And thirdly, evidence exists that already prior to the onset of hypertension specific microcirculatory alterations exist such as arteriolar rarefaction and media hypertrophy of resistance arteries $(67,147,227-229)$

The interest in the population with a blood pressure range between $120 / 80$ and $140 / 90 \mathrm{~mm}$ $\mathrm{Hg}$ is further fuelled by the recent trial of preventing hypertension (TROPHY) (121) which demonstrated a reduced progression to hypertension in these individuals by transient angiotensin antagonism.

The rising interest in this blood pressure range is mirrored by the discussion how to interprete individuals with a blood pressure between $120 / 80$ and $140 / 90 \mathrm{~mm} \mathrm{Hg}$ (9). The Seventh Joint National Committee on the Prevention, Detection, Evaluation and Treatment of Hypertension (JNC-7) introduced the term "prehypertension" $(230,231)$. In the following we will use this term. In contrast, the European Society of Hypertension guidelines 2003 (ESH 2003) subdivide this range into normal and high normal blood pressure $(12,13)$.

Indeed, the mentioned epidemiological studies which investigated prehypertension do not provide parameters for an exact individual prediction of blood pressure development. Until now it remains uncertain how long a prehypertensive individual remains prehypertensive, at which time-point in life he develops hypertension and whether the rate of progression influences the individual cardiovascular risk. However these are crucial questions if we want to improve our understanding about hypertension and if we want to evaluate treatment strategies which either aim to extend continuous antihypertensive treatment to the prehypertensive population or aim to apply prehypertensive prevention as performed by the TROPHY trial.

Therefore we created a nested case control study including prehypertensive individuals who will develop new-onset hypertension $(\mathrm{PHT} \rightarrow \mathrm{HT}$ ) or remain prehypertensive $(\mathrm{PHT} \rightarrow \mathrm{PHT})$ during a period of 4.5 years. Stable normotensive and hypertensive individuals represented positive and negative controls, respectively. In this setting we investigated potential candidates which could suit as individual predictors for the new-onset of hypertension. 


\section{Methods}

The ethics committee of the University of Leuven approved the Flemish Study on Environment, Genes, and Health Outcomes (FLEMENGHO). From June 1996 until July 2003, a random sample of the households living in a geographically defined area in northern Belgium was investigated (232). All household members $>20$ years of age were invited to take part. The participants gave informed consent. From this population we created a nested case control study investigating in total 51 subjects over a period of 4.5 years. Therefore we classified the participants in 4 groups based on their blood pressure profile according to JNC-VII $(230,231)$ at the beginning and end of the observation period: We created two prehypertensive groups. Prehypertension was defined as a systolic blood pressure of 120$139 \mathrm{~mm} \mathrm{Hg}$ systolic or $80-89 \mathrm{~mm} \mathrm{Hg}$ diastolic (11). One group of prehypertensive subjects remained prehypertensive throughout the study period (PHT $\rightarrow$ PHT, $n=8$ ), while the other group of prehypertensives developed a new-onset hypertension (PHT $\rightarrow H T, n=13$ ). Individuals remaining hypertensive $(H T \rightarrow H T, n=12)$ or normotensive $(N T \rightarrow N T, n=18)$ respectively were used as controls. Hypertension was defined as a blood pressure of $>140$ $\mathrm{mm} \mathrm{Hg}$ systolic or $90 \mathrm{~mm} \mathrm{Hg}$ diastolic or as the use of antihypertensive drugs. Normotension was defined as a blood pressure of $<120 \mathrm{~mm} \mathrm{Hg}$ systolic or $80 \mathrm{~mm} \mathrm{Hg}$ diastolic without the use of antihypertensive drugs (Figure 7.1).

At the enrolment home visit, trained nurses measured anthropometric characteristics and blood pressure. They also administered a questionnaire to collect information about each subject's medical history, smoking and drinking habits, and intake of medications. Blood pressure was the average of 5 consecutive readings. Body mass index (BMI) was weight in kilograms divided by the square of height in meters. Venous blood samples were drawn for measurement of plasma creatinine, sodium and potassium. 24-hour urine was sampled and measurements of creatinine, sodium and NOx performed. Based on the 24-hour urine and blood samples creatinine-clearance was determined. All 51 subjects were revisited after $4.5 \pm 0.2$ years. The same set of samples and measurements were performed. 
Table 7.1 Baseline characteristics assorted by blood pressure ${ }^{\wedge}$

\begin{tabular}{|c|c|c|c|c|}
\hline \multicolumn{2}{|l|}{ Label } & \multicolumn{2}{|l|}{ Controls } & \multirow{3}{*}{ 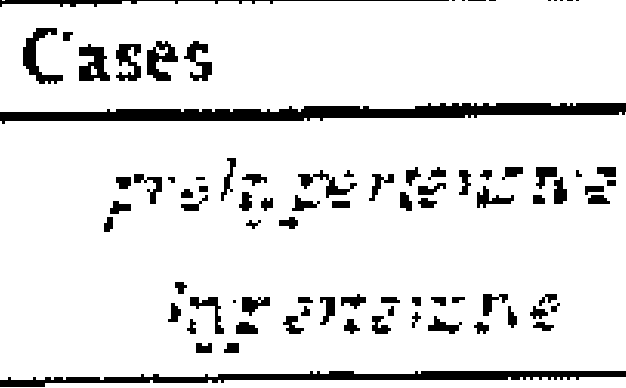 } \\
\hline 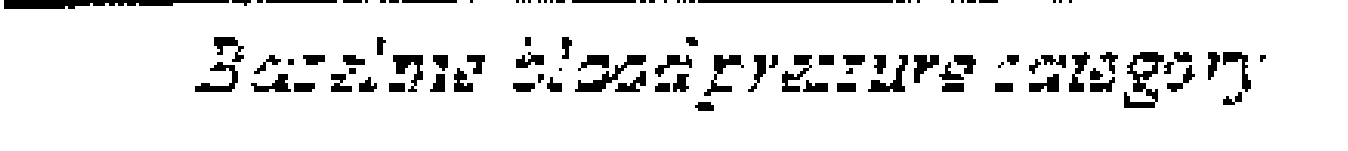 & momiar & 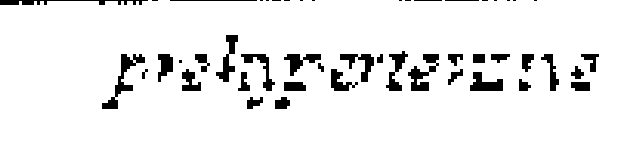 & 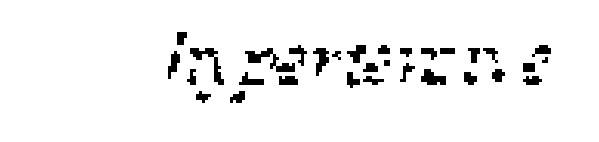 & \\
\hline 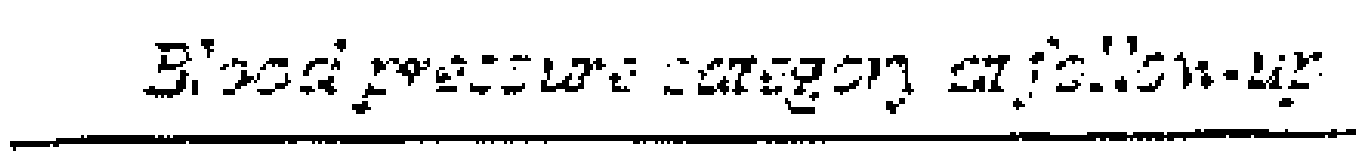 & Brmat & rometsons & $3 x+2 x=$ & \\
\hline Number & 18 & 8 & 13 & 12 \\
\hline f을 (vears) & $35.8=1.9$ & $\therefore \bar{y}=5.5$ & $\therefore-2=3.2$ & $50.8=2.37 \%$ \\
\hline Bodr: masa mder $k \mathrm{~kg} \mathrm{~m}^{2}$ ) & $2+.8=0.9$ & $263=1-$ & $2 S: 2=10^{\circ}$ & $262=1.5$ \\
\hline Systolic pressure fmm $5 g$ & $1138=12$ & $\because 33=1.1 \div$ & $1+53=3240$ & $125 \div=2 \div \div$ \\
\hline 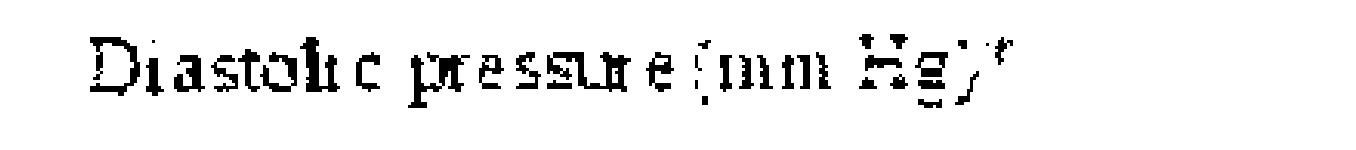 & $-0:=1 . s$ & $-9.1=2 .+4$ & $s^{-1} .1=-\cdots$ & $-5:=1.2 \cdots$ \\
\hline \multicolumn{5}{|l|}{ Serun bochemssty } \\
\hline Serum sodium imin ol $\mathrm{L}$ & $139.8=0.5$ & $140.5=0.5$ & $1 \div 0.2=8$ & $1+2.2=4.2$ \\
\hline Serum potassium inmol L? & $3.5=91$ & $\therefore 0=0.1$ & $3.5=51$ & $-2=0.1$ \\
\hline 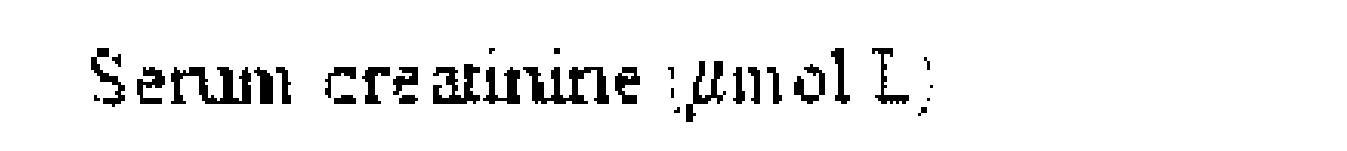 & $S 21=12$ & $952=1.2$ & $5:=3.6$ & $9 S S= \pm$ \\
\hline \multicolumn{5}{|l|}{ Grinar tes aretion rate } \\
\hline Sodium ex $\alpha$ etion $($ trmol h & $-.5=0.5$ & $\$ 1=0.6$ & $-5=05$ & $3 s=5 s$ \\
\hline Creatmine ex cren on $/ \mu m$ ol h. & $0: 5=0.93$ & $n=0=03$ & $n:=0.2$ & $0.38=1.3$ \\
\hline No: concentration in urine mel: & $205 .^{-1}=21.4$ & $198.6=23$ & $120.0=23.9 \cdots$ & $1200=22.9-+$ \\
\hline \multicolumn{5}{|l|}{ Renal function } \\
\hline Creaturine dearance $(\mathrm{m} 1 \mathrm{~m} \mathrm{~m})$ & $1199=55$ & $111 \div=6$. & $112 s=-$ & $9.8=6.5^{*}$ \\
\hline$F E_{i: 2} c_{c}$ & $065=010$ & $a^{-2}=0.0^{\circ}$ & $0 S S=0.15$ & $0.8 \div=810^{-}$ \\
\hline 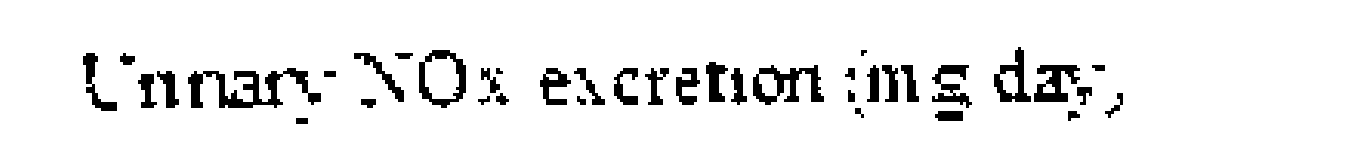 & $2 \div 0=220$ & $2630^{-2}=281$ & $1^{-} 6.1=73.6^{*+}$ & $1^{--5}=2+2 \cdots$ \\
\hline \multicolumn{5}{|l|}{ Xumber ac st th chatacterisic } \\
\hline Wonen & $12: 66$ & $3: 5$ & $5-1$ & $S(6) .5$ \\
\hline Sinioker & $+a 2$ & 19125 & 3250 & 538 \\
\hline NSAD & $2: 11.1:$ & $1: 125$ & $-(30.5)$ & $5=0$. \\
\hline Antrotpatensive dug tream ent & 00.0 & 000 & $650.0^{2} c_{j}$ & 0000 \\
\hline
\end{tabular}

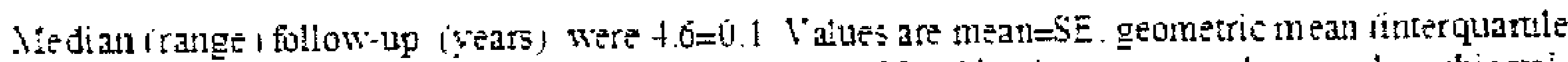
ranget or $: 10.1^{\circ}$ el of subjects. "Based on the a verage of five blood pressure readungs ar the subjects" homes

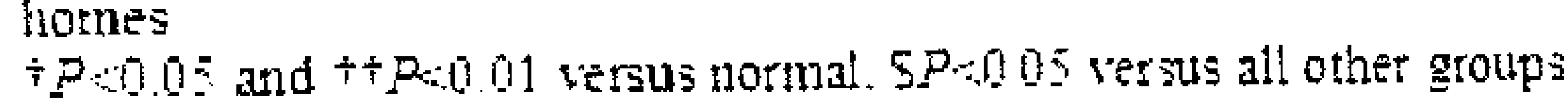

An participants, blood pressure categories relied on the guidelines of the Joint National Committee of Hypertension. Systolic and diastolic blood pressures were averages of five consecutive readings obtained at the enrollment home visit. ${ }^{B}$ Values are numbers $\pm S E M$ or number $(\%)$. Body mass index is weight in kilograms divided by the square of the height in meters. ${ }^{*}$ Significant difference $(P<0.05$ with individuals with normotensive blood pressure values. NT $\rightarrow$ NT: Normotensive at both visits. PHT $\rightarrow$ PHT:

Prehypertensive at both visits, PHT $\rightarrow$ HT: Prehypertensives developing hypertension, HT $\rightarrow H T$ :

Hypertensive at both visits

The contribution of nitrate and sodium in the diet to urinary nitrate and sodium concentrations was minimized by overnight fasting (233). The measurements were performed with a mass Spectrometry-validated HPLC method for urinary nitrate and nitrate

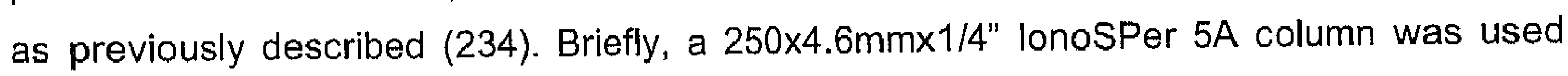
(Varian Inc.'s, Lake Forrest, CA, USA) at a wavelength of $210 \mathrm{~nm}$. As HPLC eluens 0.1M phosphate buffer at a $\mathrm{pH}$ of 4 was used. Results for nitrite and nitrate are taken together as NOx. 
We used the SAS software package (SAS Institute, Inc. Cary, North Carolina, USA), version 9.1.3, for database management and statistical analysis. We used repeated ANOVA with post-hoc Tukey-Kramer to analyze differences between the 4 groups adjusting for sex, age, $\mathrm{BMI}$ and current smoking status. All data are given in mean \pm SEM. Significance was accepted at a $P$ value of 0.05 or less.

\section{Results}

The 51 participants included $23(45.1 \%)$ men, 21 (41.2\%) prehypertensive subjects and 12 $(23.5 \%)$ hypertensive patients, of whom $6(50 \%)$ were on antihypertensive drug treatment. The 21 prehypertensive individuals included $8 \mathrm{PHT} \rightarrow \mathrm{PHT}(15.7 \%)$ and $13 \mathrm{PHT} \rightarrow \mathrm{HT}$ $(25.5 \%)$. Age at entry ranged from 18 to 71 years. Women compared with men had comparable systolic (123.3 versus $126.3 \mathrm{~mm} \mathrm{Hg}$ ), diastolic $(77.6$ versus $83.5 \mathrm{~mm} \mathrm{Hg}$; $\mathrm{P}=0.09$ ) blood pressures and pulse pressure (45.8 versus $42.8 \mathrm{~mm} \mathrm{Hg}$ ), respectively. The baseline characteristics of the four study groups were similar for BMI and sex (Table 7.1).

An overall difference of urinary NOx concentration $(\mu \mathrm{g} / \mathrm{ml})$ was apparent between the groups at the first time point $(P<0.007)$ and the second time point $(P<0.04)$, which remained after adjustment for age, sex, $\mathrm{BMI}$ and current smoking status $(P<0.01$ or $P<0.03$, respectively). None of the adjustment factors showed significance. There was no time effect seen for urinary NOx concentration (Wilks' Lambda 0.55). The adjusted repeated analysis for all samples (time point one and two) to test the hypothesis for between group effects demonstrated a high significance $(P<0.0006)$. To assess the potential predictive values of urinary NOx concentration we investigated the first time point for within group effects regarding blood pressure development. We demonstrated that urinary NOx concentration of normotensive individuals was significantly higher as compared to hypertensives $(P<0.009)$ and $\mathrm{PHT} \rightarrow \mathrm{HT} \mathrm{P}<0.004)$. Secondly, $\mathrm{PHT} \rightarrow \mathrm{PHT}$ demonstrated comparable urinary NOx as normotensives, no differences between $\mathrm{PHT} \rightarrow \mathrm{HT}(\mathrm{P}=0.07)$ and significantly higher values as hypertensives $(P<0.03$, Figure $7.2 a)$.

There was a general tendency in the subject to lower NOx values at the end of the observation period which remained after adjustment $(P<0.01)$. The changes in urinary NOx concentration during the observation period varied between the groups. We observed the strongest reduction in prehypertensive individuals. $\mathrm{PHT} \rightarrow \mathrm{HT}$ showed significantly enhanced reduction as compared to hypertensive individuals $(P<0.05)$. 
An overall difference of 24-hour urinary NOx excretion (mg/day) was apparent after adjustment between the groups at both time points $(P<0.0004)$ without time effect (Wilks' lambda 0.71 ) and the second time point $(P<0.002)$. Sex was a relevant adjustment factor $(P<0.009)$. To asses the potential predictive values of 24 -hour urinary NOx excretion (mg/day) we investigated the first time point for within group effects regarding blood pressure development. We demonstrated that 24-hour urinary NOx excretion (mg/day) of normotensive individuals was significantly higher as compared to hypertensives $(P<0.0002)$ and $\mathrm{PHT} \rightarrow H \mathrm{HT}(\mathrm{P}<0.002)$. Secondly, $\mathrm{PHT} \rightarrow \mathrm{PHT}$ demonstrated comparable urinary NOX as normotensives, no differences between $\mathrm{PHT} \rightarrow \mathrm{HT}(\mathrm{P}=0.10)$ and significantly higher values as hypertensives $(P<0.02$; Figure $7.2 \mathrm{~b}$ ).

Figure 7.1 Blood pressure development according to the baseline group assignment
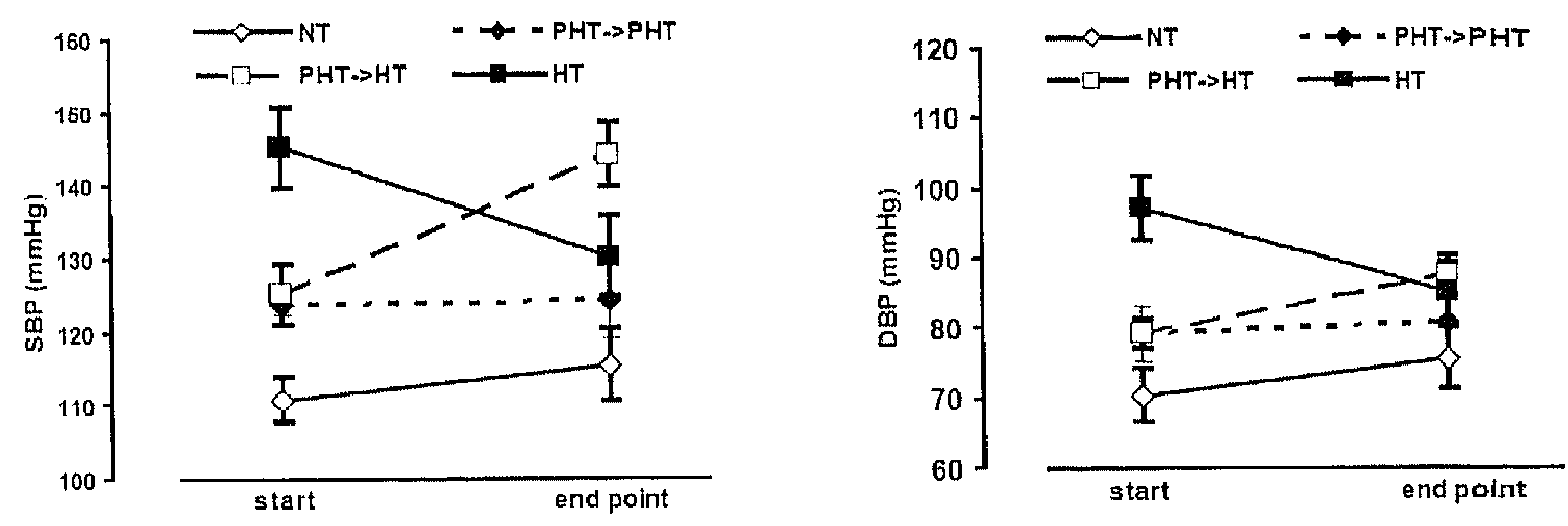

Figure 7.1a demonstrates baseline distribution of the systolic blood pressure according to the groups NT, $\mathrm{PHT} \rightarrow \mathrm{PHT}, \mathrm{PHT} \rightarrow \mathrm{HT}$ and $\mathrm{HT}$ and the systolic blood pressure values after 4.5 years according to the baseline group assignment. Figure $7.1 \mathrm{~b}$ demonstrates baseline distribution of the diastolic blood pressure according to the groups NT, PHT $\rightarrow$ PHT, PHT $\rightarrow H T$ and $H T$ and the diastolic blood pressure values after 4.5 years according to the baseline group assignment.

NT $\rightarrow$ NT: Normotensive at both visits, PHT $\rightarrow$ PHT: Prehypertensive at both visits, PHT $\rightarrow H T:$ Prehypertensives developing hypertension, $\mathrm{HT} \rightarrow \mathrm{HT}$ : Hypertensive at both visits

There was a general tendency in the subjects towards less NOx excretion at the end of the observation period which remained after adjustment $(P<0.001)$. The changes in urinary NOx excretion during the observation period did not significantly vary between the groups. 
Figure 7.2 Adjusted ${ }^{\mathrm{a}}$ baseline measurements and time related change (\%) according to the baseline assignment ${ }^{\mathrm{b}}$

A

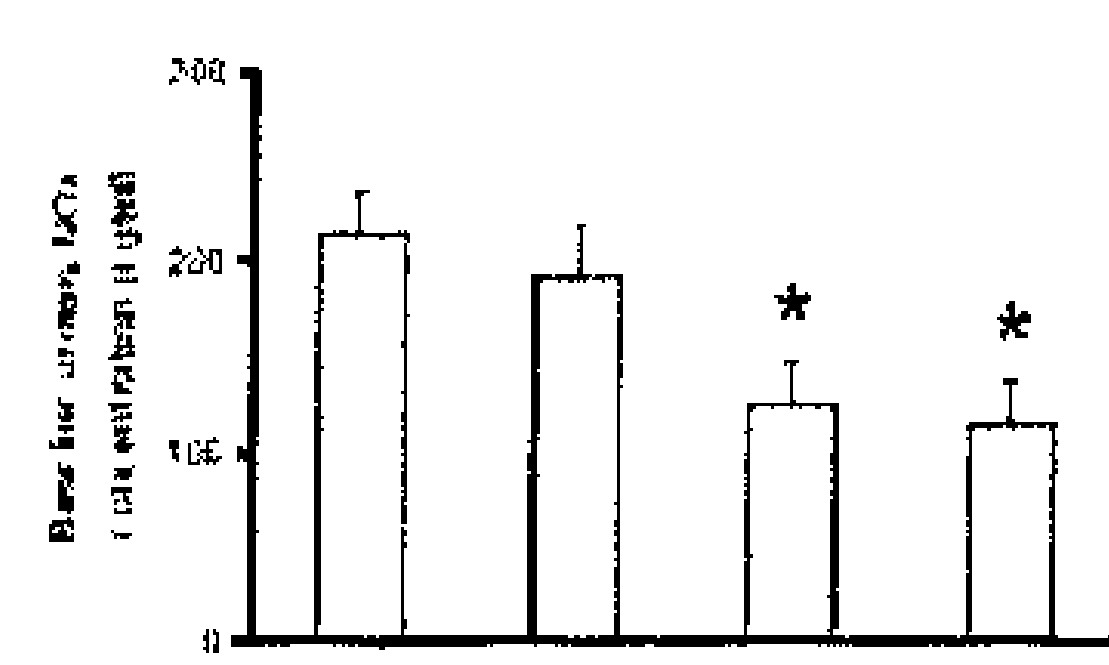

NI PHI HII PHI A H III

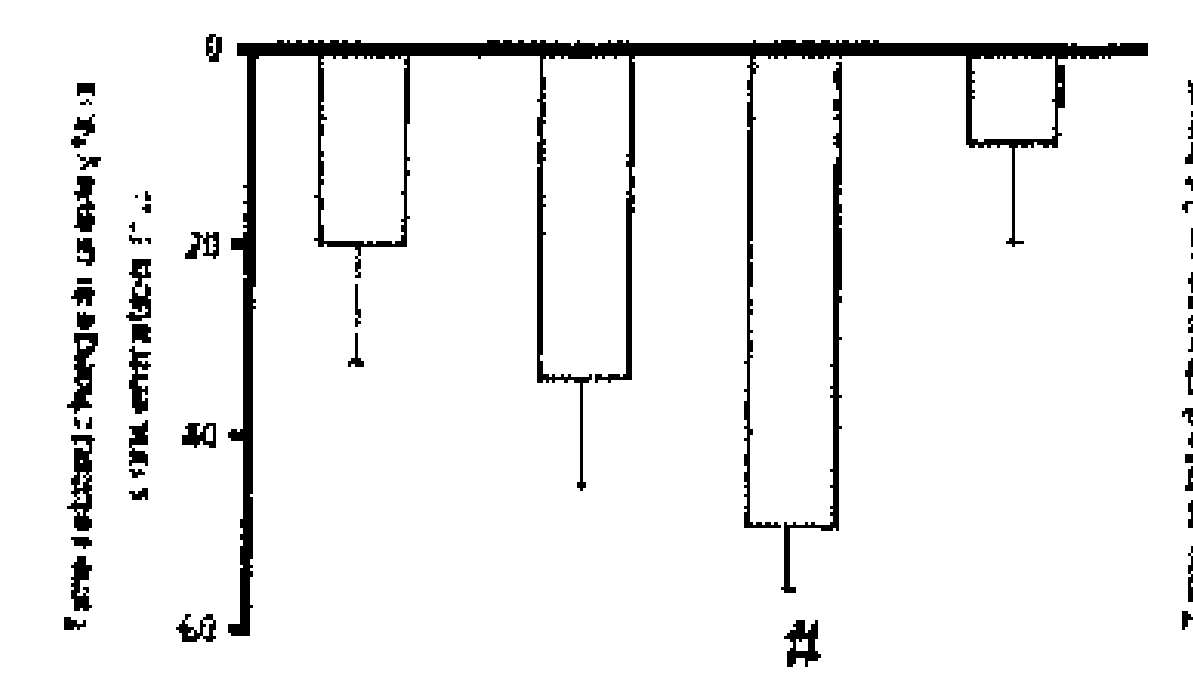

B

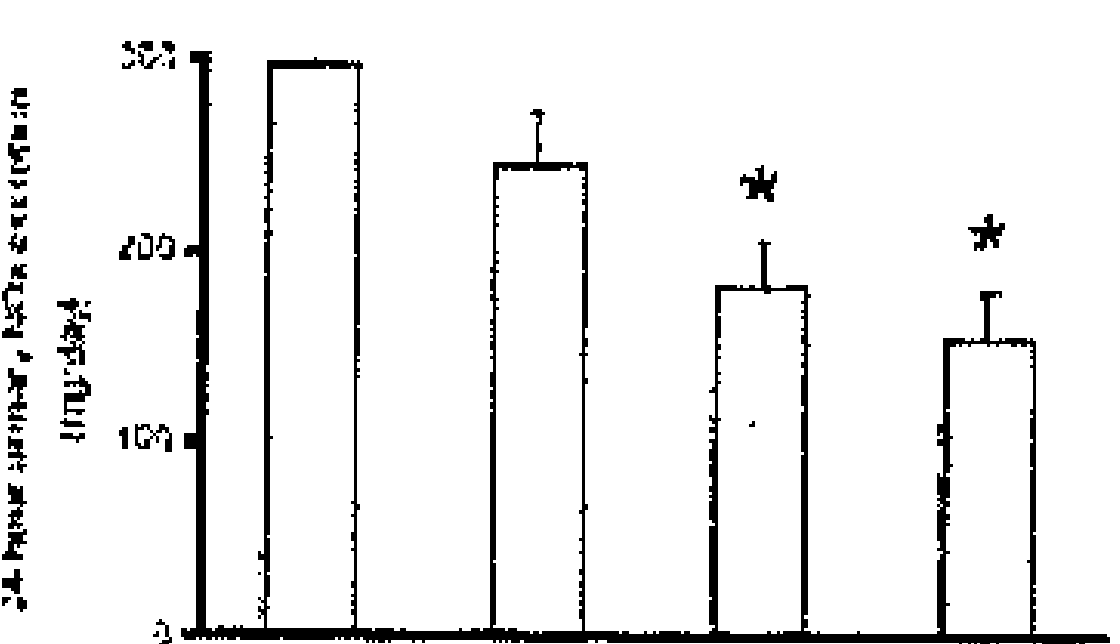

NI PHI. . HT PHT ...HT HT

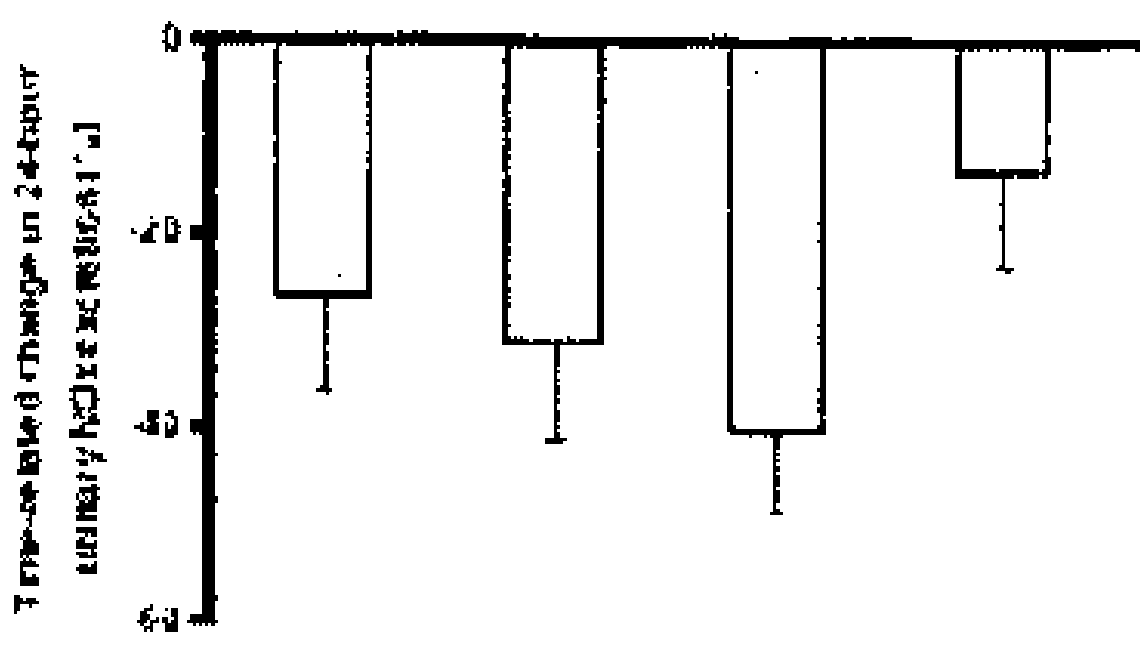

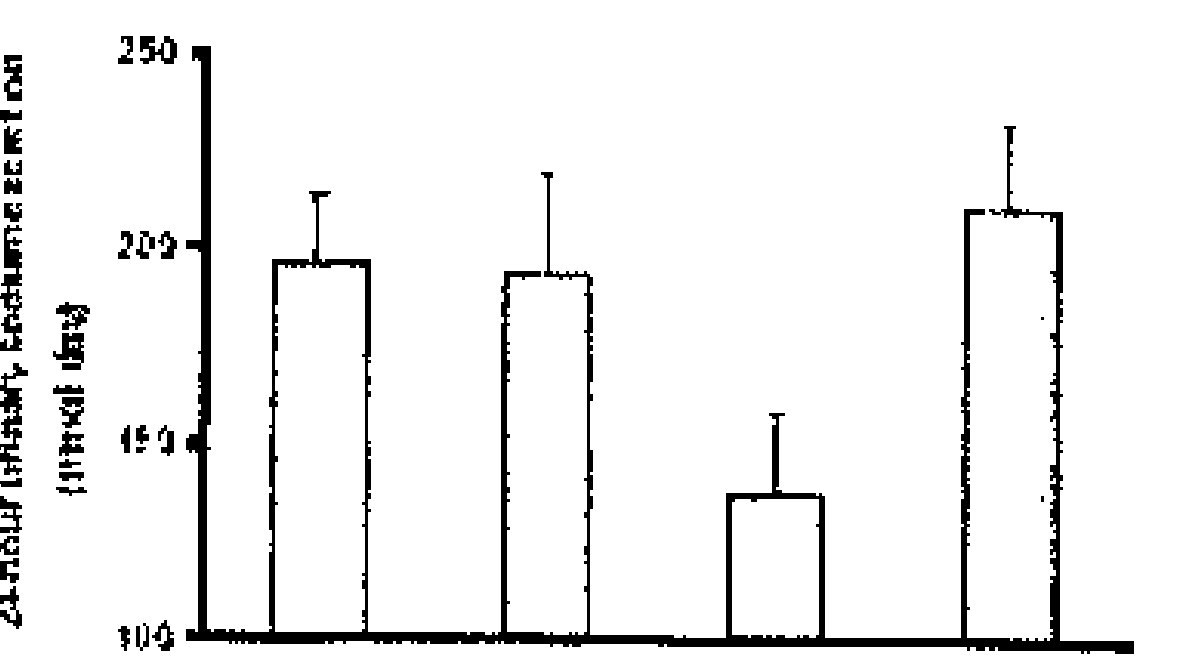

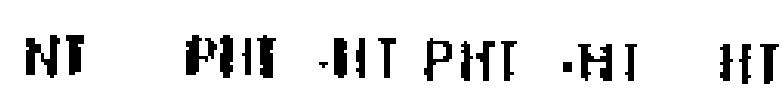

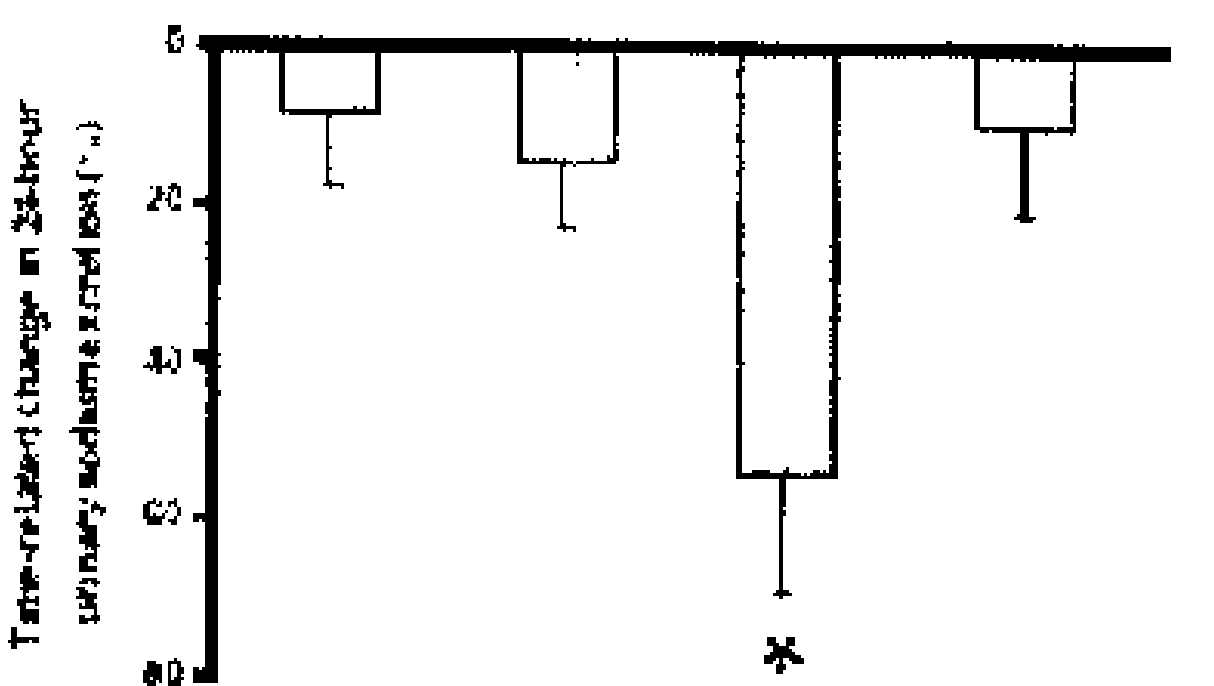

D

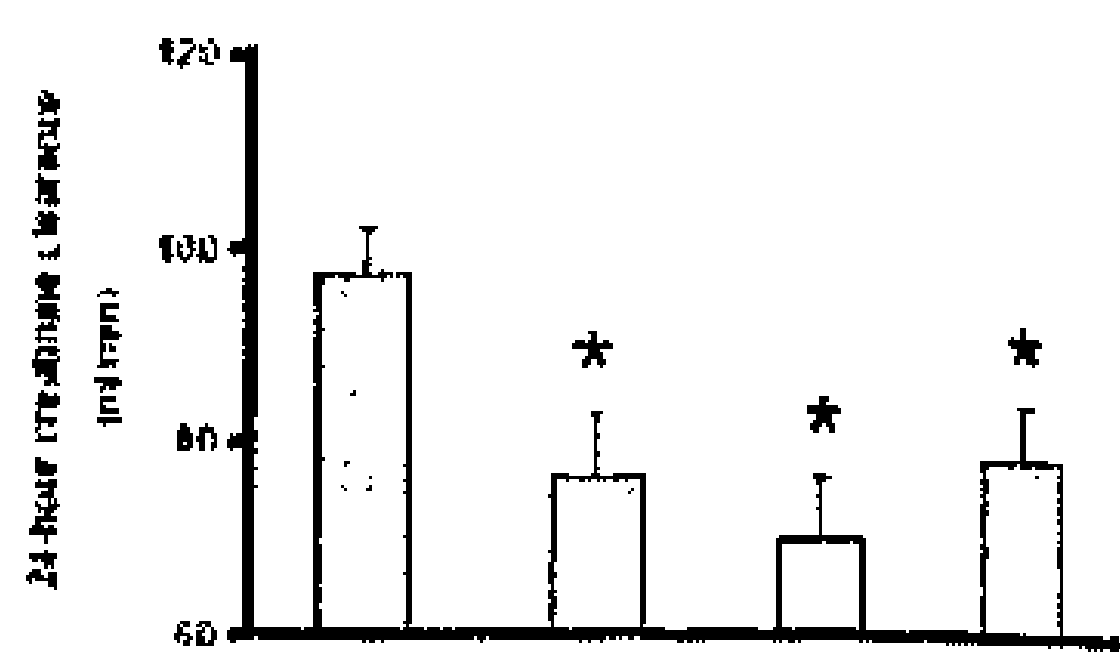

NI PHII ...HI PHI...HT HT

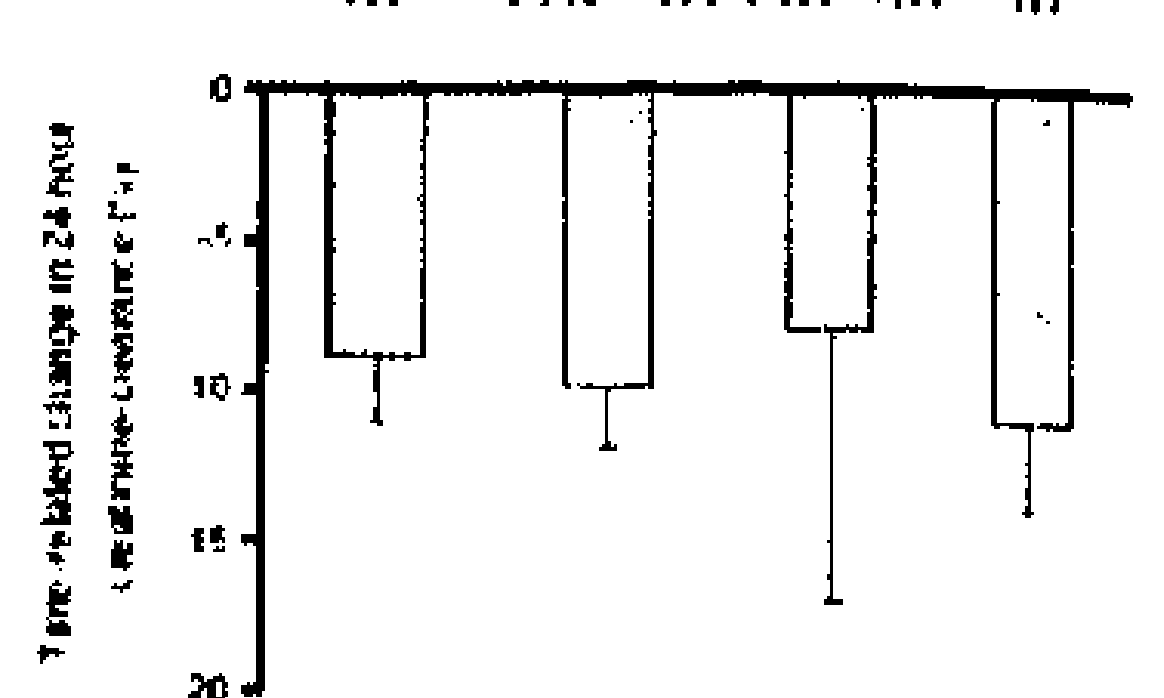

Figure 7.2A demonstrates a reduction of adjusted urinary NOx concentration in $\mathrm{PHT} \rightarrow \mathrm{HT}$ and $\mathrm{HT}$ as compared to NT $\left({ }^{\star} \mathrm{P}<0.01\right)$. A time related reduction of the values is obvious in all groups, but in PHT $\rightarrow H T$ as compared to $\mathrm{HT}$ this was significantly enhanced $(\# \mathrm{P}<0.05)$.

Figure 7.2B demonstrates similar pattern for 24-hour NOx excretion in $\mathrm{PHT} \rightarrow \mathrm{HT}$ and $\mathrm{HT}$ as compared to $N T\left({ }^{*} \mathrm{P}<0.01\right)$. The time related changes show a comparable tendency, but do not reach significance.

Figure $7.2 \mathrm{C}$ shows a borderline reduced sodium excretion at baseline in PHT $\rightarrow H T$ as compared to all other groups. The time related change demonstrates selectively in $\mathrm{PHT} \rightarrow \mathrm{HT}$ an increase sodium excretion according to baseline results which is significant against NT and $H T\left({ }^{*} \mathrm{P}<0.05\right)$.

Figure 7.2D shows the reduced creatinine clearance in both prehypertensive groups and hypertensive individuals. A time related reduction of the values is obvious in all groups $\left({ }^{*} P<0.05\right)$. No differences according to the group assignment are apparent.

aSerum creatinine, creatinine clearance and sodium excretion were adjusted for age, sex and BMI. Urinary NOx values were additionally adjusted for current smoking status. Values are numbers \pm SEM or number (\%). NT $\rightarrow$ NT: Normotensive at both visits, PHT $\rightarrow$ PHT: Prehypertensive at both visits, PHT $\rightarrow$ HT: Prehypertensives developing hypertension, HT $\rightarrow H T$ : Hypertensive at both visits

An overall difference of creatinine clearance $(\mathrm{ml} / \mathrm{min}$ ) was apparent between the groups at the first time point $(P<0.0005)$ and the second time point $(P<0.0005)$, which remained after adjustment for age, sex, $\mathrm{BMI}$ and current smoking status $(\mathrm{P}<0.02$ or $P<0.03$, respectively). Age $(P<0.01)$ and $\mathrm{BMI}(P<0.004)$ remained after adjustment significant covariates. There was no time effect evident for creatinine clearance (Wilks' Lambda 0.76). The adjusted repeated analysis for all samples (time point one and two) to test the hypothesis for between group effects demonstrated a high significance $(P<0.03)$. To asses the potential predictive value of creatinine clearance we investigated the first time point for within group effects regarding blood pressure development. We demonstrated that 24-hour creatinine clearance of normotensive individuals is significantly higher as compared to hypertensives $(P<0.03)$ 
and both prehypertensive groups $(P<0.03)$. No difference was evident between the two prehypertensive groups or hypertensive individuals, respectively (Figure 7.2c).

The creatinine-clearance at the end of the observation period was significantly lower as compared to the starting point $(P<0.01)$. The changes during the observation period did not significantly vary between the groups. After adjustment for age, sex and BMI no significant group effect was evident for plasma creatinine ( $\mu$ mol/l, $P=0.52)$. Sex was a significant covariate $(P<0.0007)$. After adjustment for age, sex and BMI no significant group effect was evident for 24 -hour sodium excretion $(P=0.37$ ). However, baseline sodium excretion was not significantly reduced in prehypertensives at baseline as compared to normotensive $(P=0.10)$ and hypertensive individuals $(P=0.08)$. Sex was a significant covariate $(P<0.03$, Figure $7.2 \mathrm{~d})$.

There was a not significant tendency in the PHT $\rightarrow \mathrm{HT}$ group towards lower sodium excretion at the start of the observation period $(P=0.09)$. This fully normalized at the end of the observation period. This resulted in a significant change of sodium excretion varied between the groups comparing start and end of the observation period. PHT $\rightarrow H T$ demonstrated a significantly enhanced change regarding sodium excretion as compared to normotensives and hypertensives $(P<0.05)$, while the difference to $P H T \rightarrow P H T$ remained non-significant $(P=0.08)$.

\section{Discussion}

We demonstrate that $\mathrm{PHT} \rightarrow \mathrm{HT}$ during a period of 4.5 years are characterized by a reduced urinary NOx such as hypertensives and temporary reduced sodium excretion. In contrast. $\mathrm{PHT} \rightarrow \mathrm{PHT}$ demonstrate normotensive values for urinary NOx and sodium excretion. Therefore urinary NOx and sodim excretion could have the potential to predict new-onset hypertension in prehypertensive individuals as it discriminates PHT $\rightarrow$ PHT and PHT $\rightarrow$ HT. To further investigate the predictive value of these factors prospective studies are warranted.

In the past decades several authors investigated the development of hypertension in experimental rat studies. These authors evidenced that the development of hypertension is closely linked to renal causes $(53,54,57,58,81)$. Underlying theories propose that either a primary renal microvascular disease $(235)$ or a relative defect in sodium excretion in 
response to alterations in blood pressure is evident (shift in pressure natriuresis curve) (49, 50 ). Taking these aspects into account, the spontaneously hypertensive rat (SHR) is during the period of steepest blood pressure rise characterized by enhanced renal vascular resistance and sodium reabsorption and reduced glomerular filtration rate (GFR) and NO availability $(236,237)$. We hypothesized that this period in SHR might be comparable with the prehypertensive period prior new-onset of hypertension. Therefore we chose plasma creatinine and creatinine clearance as marker for renal function and GFR, sodium excretion as indirect marker for sodium reabsorption and urinary NOx as marker for NO bioavailability $(233,238)$. We investigated these factors in a nested case control study.

The first new finding of this study is that low urinary NOx values are closely associated with the $\mathrm{PHT} \rightarrow \mathrm{HT}$ and hypertensive individuals during a period of 4.5 years. This association is independent of age, sex, BMl and current smoking status (239-241). A full discrimination is possible between normotensives and $\mathrm{PHT} \rightarrow \mathrm{HT}$. Additionally, a full discrimination is possible between hypertensives and $\mathrm{PHT} \rightarrow \mathrm{PHT}$. The discrimination between the two prehypertensive groups remains borderline, which is largely dependent on the small sample size of these two groups. Therefore our findings suggest that urinary NOx excretion is a potential predictor for the new-onset of hypertension in prehypertensive individuals.

A number of recent manuscripts demonstrated that urinary NOx is decreased in hypertensives as compared to normotensives $(233,241,242)$ and even that prehypertensives demonstrate intermediate values (240). Regarding the longitudinal relevance of this marker no human data were available yet. However, animal data evidenced that NO bioavailability is hampered in SHR $(167,243,244)$. Secondly, restoring NO during the early period in life attenuates blood pressure development in young SHR (245). Whether the origin of the hampered NO availability is related to systemic or renal effects cannot be answered by this study. Regarding the method of NOx determination, we calculated NOx as sum of nitrite and nitrate in urine using a HPLC method as previously described (234). The method is validated by mass spectrometry and more precise than the common Griess reaction. In context with common literature we interpret urinary NOx as marker for NO availability (234). 
The second major finding is that the sodium excreton is temporanly hampered in PHT. .HT. Only PHT ..HT showed during the period of prehypertension a tendency towards retuced sodium excretion. This finding alone did not reach statstical signficance. However. comparing the change of sodium exretion between both observatonal time points we found a statistrcaliy significant increase of sodium excretion restricted to the PHT. .HT group. These findings reflect the basic rdea of the Borst-Guyton hypothesis whoh expects increased sodium reabsorption in PHT .HT pror onset of hypertension resulting in a reduced sodium excretion (49. 50) PHT .PHT did nether demonstrate reduced sodium excretion at baseline nor a comparable time-related change. This suggests that the transient reduction of sodum excretion is specific for PHT ...HT. Beyond this point it might be speculated whether the transient change in sodium homeostasis reflects an active mechanism in the development of hypertension. This idea includes that active mechanisms can be theoretically antagonized, thereby preventing the development of hypertension as recently demonstrated by the TROPHY trial (121).

Blood pressure is proposed to travel with the kidney $(54,56,246)$ and renal function is known to correlate with blood pressure and cardiovascular mortality (247). Therefore we tested the hypothesis whether serum creatinine or creatinine clearance are associated with the PHT $\rightarrow H T$. Serum creatinine does not differ between the groups after adjustrnent for age, sex and BMI. In contrast, creatinine-clearance demonstrated significant differences between the groups. Creatinine-clearance is similarly reduced in reduced hypertensive and prehypertensive individuals. Therefore creatinine-clearance does not differentiate between $\mathrm{PHT} \rightarrow-\mathrm{HT}$ and $\mathrm{PHT} \rightarrow \mathrm{HT}$ during a period of 4.5 years.

The present study must be interpreted within the context of its limitations. First, the sample size is small. Secondly, measurements for NOx and sodium were based on 24-hour measurements without introducing standard diets to the participants. Thirdly, to prove causality between the factors prospective studies are warranted. In conclusion, these results give further insight in the development of hypertension and lead us to the new working hypothesis that those subjects are developing new-onset hypertension who are prehypertensive and demonstrate low urinary NOx and sodium excretion. These results will stimulate prospective studies to investigate the role of these factors for a prediction of newonset hypertension. 


\section{GENERAL DISCUSSION AND CONCLUSIONS}

\section{Potential of prehypertensive treatment in the SHR \\ Blood pressure lowering}

There is increasing evidence that transient prehypertensive treatment in SHR delays blood pressure development $(84,103,104,111,117)$. This delay leads to a prolonged blood pressure reduction. One key result of this thesis is that the blood pressure lowering is not persistent throughout life. A blood pressure lowering effect based on one month transient prehypertensive treatment persists for approximately 9 months after drug withdrawal in SHR. During adulthood blood pressure slowly increases reaching hypertensive control values. The prolonged lower blood pressure levels in prehypertensively treated SHR should reflect less deleterious effects of hypertension on target organs. We cannot extrapolate the period of 9 month in SHR to the human situation.

\section{Target organ protection}

Indeed, recent publications demonstrate that cardiac and glomerular target organ damage are reduced after transient prehypertensive treatment as long as the blood pressure remains reduced $(105,248)$. We confirmed these results in our long-term study. However we furthermore asked the clinically relevant question what happens to the target organ damage after blood pressure has again reached untreated SHR values. Interestingly, following transient prehypertensive treatment during the time window of 4-8 weeks of age, target organ damage remains reduced up to the advanced age of 72 weeks. Thus the second main finding of this thesis is that target organ damage remains reduced after transient prehypertensive treatment up to 72 weeks of age, an age at which blood pressure has returned to values comparable to those in non-treated SHR.

The question has to be asked why the damage remains reduced up to advanced age despite the rise of blood pressure during adulthood and therefore hypertensive blood pressure values for approximately 8 months. In this context two potential answers exist: Firstly, the prolonged blood pressure reduction during the first 10 months of age decelerated the accumulation of damage. Therefore, the total amount of cardiovascular damage at the time point of similar blood pressure values between transiently prehypertensive and untreated SHR is reduced in the group of prehypertensively treated SHR. This implies that the once created advantage regarding less target organ damage in the group of 
prehypertensively treated SHR remains. This could explain why we observed a loss of cardiac and glomerular function in prehypertensively treated SHR approximately 3 month later than in untreated SHR. However several aspects described in Chapter THREE cannot be explained by this effect only. For example, the extent of interstitial cardiac fibrosis is extremely low. It does not exceed normotensive values although blood pressure levels were higher than in normotensive rats throughout the whole life. This raises the question whether, besides the blood pressure lowering effect, other blood pressure independent factors are apparent after transient prehypertensive treatment.

If we want to extrapolate these data to the human situation, we should consider not only investigating the blood pressure reduction induced by prehypertensive treatment. Additionally we should measure whether the early target organ damage such as left ventricular hypertrophy or microalbuminuria remain reduced. The investigation should be continued even if no blood pressure reduction is apparent anymore.

\section{Blood pressure independent effects}

This leads us to the second potential answer: Blood pressure independent mechanisms are involved in the attenuation of target organ damage. Chapter FOUR may contain a clue. The major finding in Chapter FOUR is that after prehypertensive treatment vascular structure remains largely unaffected up to advanced age of 72 weeks of age, but function is clearly improved. No long-term protection against vascular hypertrophy is apparent in the vasculature. However, the general ability to constrict is enhanced in prehypertensively treated 72 weeks old SHR. Furthermore, the NO dependent vasorelaxation is enhanced in these rats as compared to untreated SHR. These findings are comparable to recent results of continuous RAS inhibition, which have been explained by the fact that continuous RAS inhibition has a blood pressure independent effect on reducing vascular aging (162). Taking into consideration that we see the same pattern after transient prehypertensive treatment as compared to continuous RAS inhibition as described by others (162) we suggest that transient prehypertensive treatment decelerates aging in the vasculature of SHR. Thus, the power of vascular SMC is greater after transient prehypertensive treatment resulting in a stronger vascular contraction. In endothelial cells it seems that the NO handling is shifted. Although the endothelial NO amount remains unchanged we demonstrated higher concentrations of eNOS in compartments of the cell which seem to be the Golgi-complex 
(170). Therefore eNOS and resulting NO are protected against oxidative stress which can be found at the cell membrane (NADPH oxidase) or intracellularly $(167,249)$.

The aging process is known to be accelerated in SHR (173). Cells of the cardiovascular system show an increased rate of aging as compared to normotensive controls (176). However, the question remains how to define the aging process. Aging can be defined in many ways. Cell-turnover can be enhanced (176), extracellular matrix proteins can accumulate (229), and intracellular non-recyclable products can accumulate (198). Genes can age by methylation (250), proteins by glycosylation (251), and lipids by peroxidation (252). In literature the most common parameter for aging is cell-turnover. This process results from the balance of cell proliferation and apoptosis (177). As one consequence, enzymes which are called telomerases cut each cell cycle small pieces from the ends of the DNA strands which represent the telomeres (193). Telomeres therefore decrease their length every cell cycle. If the cell-turnover is enhanced, telomerases have more chances to reduce telomeres, thereby subsequently reducing telomere length. This leads to smaller telomere length in cells with high cell-turnover as compared to low cell-turnover, which is commonly interpreted as advanced aging $(174,253)$.

In the first part of Chapter FIVE we demonstrated that cell proliferation is increased in young SHR. This process is not blood pressure, but RAS dependent and TGF mediated (187). Therefore one aspect of aging is regulated independently of blood pressure. One crucial step in cell proliferation is the $G 1$ phase $(183,195)$. This phase is characterized by a holoenzyme formed from cyclins and cyclin-dependent kinases which together are able to phosphorylate a protein which finalizes the G1 phase and brings the cell in the following stage. We investigated how far the G1 phase is affected after transient prehypertensive treatment in renal tissue of 72 weeks old SHR. We found that gene expression of the major components of the $\mathrm{G1}$ - the cyclins and cyclin dependent kinases - remain strongly downregulated up to advanced age. This is associated with a lower rate of phorphorylation of the target protein. Therefore we can conclude that the cell proliferation is reduced involving a reduced cell proliferation in the $\mathrm{G} 1$ phase.

Whether in humans an attenuated aging process by transient prehypertensive treatment is of relevance has to be determined in the future. However, it might be of relevance as in particular elderly suffer from cardiovascular morbidity. 


\section{A shift in RAAS sensitivity as a concept for prolonged target organ protection}

The crucial question remains why a blood pressure independent effect remains up to 16 months after drug withdrawal (104). The medication cannot act directly after drug withdrawal as the half-life of the medication is, depending on the drug, 12-24 hours (254). Successful transient prehypertensive treatment is largely observed with drugs inhibiting the RAS. This leads us to the conclusion that the RAS is of major importance in this treatment. Furthermore evidence exists that the RAS is highly activated in young $\operatorname{SHR}(97,98,255)$. This activation finds its maximum during a time frame of 4-8 weeks after birth (97). This coincides with the treatment period leading to prolonged effects. Therefore we hypothesize that during this period the set-point for the RAS in SHR is determined and that transient RAS inhibition might shift the level of the set-point. Our hypothesis is confirmed by our experiments demonstrating that in transiently prehypertensively treated SHR more angiotensin II is needed than in untreated SHR to reach the half maximal blood pressure increase. However, basal angiotensin levels are not altered by the treatment. Therefore we conclude that besides the prolonged blood pressure lowering effect a shift in angiotensin sensitivity is involved in the sustained effects up to advanced age.

Investigating the angiotensin sensitivity in prehypertensive humans before and after transient treatment should be therefore a goal for the future. A recent technique was described by Houben et al. (256) demonstrating an non-invasive technique to investigate the vascular reactivity to angiotensin II in vivo. This technique would allow investigating the idea of activated RAAS in prehypertensives and a resetting of the RAAS with prehypertensive treatment in a non-invasive manner.

\section{Structural alterations in the renal medulla}

The shift in angiotensin sensitivity alone does not explain the initial blood pressure lowering until adulthood. Therefore we were interested in the underlying mechanism of the prolonged blood pressure lowering.

The kidney is thought to play a major role in the development of hypertension $(49,50)$. The renal medulla modulates long-term blood pressure regulation $(72,257)$. This can be mediated either by tubular cells or the renal medullary microcirculation. The renal medullary microcirculation largely consists of endothelial cells. Pericytes take over the job of vascular SMC contracting vasa recta which control the blood flow towards the tip of the medulla (206). Therefore they are involved in the sodium homeostasis. We initially found a difference 
in the structure of the renal medullary microcirculation of the SHR. We showed that the renal medulla of SHR has a 4 times higher density of pericytes as WKY. We hypothesized that the increased number of pericytes can lead to an increased vasoconstriction, increased resistance and reduced local blood flow. This would lead to increased local sodium reabsorption which would affect sodium homeostasis and long-term blood pressure regulation. Consequently, a reduction of pericytes would reduce the entire process and lead to persistent lower blood pressure values. Therefore our data are strengthening the idea of Cowley that medullary aspects play a role in the development of hypertension $(72,205$, 257). The group of Cowley is focusing on a functional effect based on a shift in angiotensin and NO which affects the local tubulovacular crosstalk $(258,259)$. Our data further point into the direction that already at young age a structural renal medullary alteration is present which would lead to an even stronger effect on the tubulovascular crosstalk since more contractile cells are present in the medulla of SHR as compared to WKY. Therefore we pinpoint the attraction of the renal medulla originated by Cowley to the renal medullary pericyte as medullary actor which determines blood pressure development.

\section{Renal medullary pericytes regulate blood pressure}

We performed transient prehypertensive treatment in SHR and found the number of pericytes reduced after treatment. In order to prove that the number of pericytes plays a major role in blood pressure regulation and how the reduction takes place, we modified transient prehypertensive treatment. Under the premise that the reduction of pericytes by transient prehypertensive treatment is mediated by apoptosis we added a pan-caspase inhibitor to the common ARB. Caspases are involved in apoptosis $(221,260)$. Treatment with pan-caspase-inhibitors reduces apoptosis in animals and humans without any direct blood pressure effect of the drug $(220,261)$. The combined treatment in prehypertensive SHR led to increased pericyte number and blood pressure as compared to ARB monotherapy. This provides further evidence that the pericyte number modifies blood pressure and that apoptosis is involved in this process. To check why apoptosis occurred in pericytes of SHR we performed additional experiments. Pericytes are known to undergo apoptosis under hypertonic stress (217). Indeed, we found a higher sodium concentration in the tip of the medulla of transiently treated SHR as compared to untreated SHR. Recent literature suggests that hypertonic stress induces GSK mediated apoptosis (217). In transiently treated SHR we demonstrated an increased level of phosphorylated GSK in the 
renal medula and furthermore showed that a combined treatment with an ARB and a GSK inhibitor reverses the effects of ARB monotherapy. We conclude that the increased medullary pericyte number in SHR is causally involved in the increased blood pressure levels in these rats. Furthermore, transient prehypertensive treatment reduces the number of pericytes by a process which finally leads to apoptosis of pericytes. This coincides with the blood pressure lowering. Selective inhibition of the apoptotic process in combination with transient prehypertensive treatment not only reverses pericyte apoptosis, but also blood pressure lowering effects. In context with the theory of Cowley this brings us to the conclusion that the pericyte and its contractile action $(215,262)$ is not only involved in the development of high blood pressure but can also protect against hypertension development.

\section{Pharmacological Perspective}

Taking into consideration the effects of prehypertensive treatment on medullary blood pressure homeostasis and blood pressure independent mechanisms such as aging, the RAAS plays a major role $(263,264)$. The prolonged blood pressure lowering seems to involve a sodium dependent apoptosis of pericytes which is induced by both ARBs and aldosterone antagonists. In combination with results of others demonstrating effects on renal vasculature and sodium handling, we put forward that all RAAS-inhibitors which can affect the sodium dependent pericyte apoptosis could lower blood pressure for a prolonged time period. $(154,265-268)$. Beside the direct pharmacological action we also observed a prolonged shift in angiotensin sensitivity. We found these effects in prehypertensively ARB and aldosterone-antagonist treated SHR. This again lead us consider that all RAASinhibitors should be similarly effective, but might be differentially accentuated by the tissue availability of the drug. However, we think that the effect of the aldosterone antagonist is rather mediated by the indirect action on the AT1R and angiotensin action (154) since the results on blood pressure lowering are less than after ARB.

Renin-inhibitors could be an interesting choice in the future. Although no data exist based on our results we hypothesize that renin-inhibition might lead to identical actions. This idea finds its basis also in data of Henrich who demonstrated that renin is overactivated in young SHR (189).

Theoretically, drugs which induce apoptosis of renal medullary pericytes should lead to prolonged lower blood pressure. Drugs which induce apoptosis in pericytes independently of the RAAS might reduce blood pressure. This idea also implies that drugs which protect renal 
medullary pericytes might even increase blood pressure in the long-term. This is particularly relevant for drugs inhibiting GSK such as the antiepileptic drug valproate. In this context it might be of interest to investigate in how far hypertensive patients with epilepsy on valproate show higher blood pressure level as with other drugs such as carbamzepine $(225,269)$.

Regarding the long-term protection of the different treatments, the ARB was superior to the aldosterone antagonist. Cardiac and renal functions were better with the ARB. Long-term vascular function and blood pressure independent factors as renal aging were similar following the treatments. This lead us conclude that the ARB has advantages as it represents a better end organ protection.

Taking blood pressure reduction and end organ protection together our data demonstrate that more relevant protection is reached with an ARB as compared to an aldosterone antagonist. This does not exclude that an ACE-inhibitor and renin-antagonist are equally potent. It might even be even considered that an ACE-inhibitor activates additional treatment pathways as compared to an ARB. The work of O'Sullivan directs to the bradykinin pathway as combined pharmacological prehypertensive treatment with an ACE-inhibitor and bradykinin inhibitor does not reduce blood pressure in SHR for a prolonged time period (113-115).

Therefore future experimental and clinical trials have to study which prehypertensive drug treatment is most effective in regards of blood pressure lowering and target organ protection.

\section{When to perform transient prehypertensive treatment in humans - a translational approach}

\section{Marker of the development of hypertension}

During the investigation of the renal medullary alteration we found that the structural alterations coincide with functional alteration, such as increased medullary vasoreactivity $(258,259)$ resulting in reduced urine production, increased sodium reabsorption and reduced NO bioavailability as seen by low urinary nitrite measures upon angiotensin stimulation $(243,245,270)$.

We predicted that the same renal medullary alterations are apparent in prehypertensive and, later, hypertensive humans. We hypothesized that under these conditions the same functional characteristics could develop in prehypertensives developing hypertension. In contrast, subjects who do not develop hypertension should not show these characteristics. 
This could lead to an identification of individuals prone to hypertension before the onset of hypertension.

\section{Performing a case control study}

We performed a nested case control study covering a period of 4.5 years including otherwise healthy subjects who we classified into four groups: prehypertensives developing new-onset hypertension, prehypertensives remaining prehypertensive, normotensives and hypertensives. We demonstrated that urinary NOx excretion at baseline was significantly reduced only in prehypertensives who later developed new-onset hypertension. Normotensives and prehypertensives remaining prehypertensive showed higher urinary NOx values at baseline. This is the first longitudinal description of the effects of urinary NOx in prehypertensives. Secondly, we described an attenuated sodium excretion only before new-onset hypertension. This strengthens the Borst-Guyton hypothesis. As this phenomenon was restricted to prehypertensives developing hypertension this might also point to the future question whether the development of hypertension is a disease by itself. Then, the elevated blood pressure would represent only the result of the original disease (development of hypertension).

It is important to notice that we only describe associations in this chapter. Therefore prospective studies without nested case control style are warranted to answer the question whether urinary NOX and sodium excretion together with blood pressure can be used as predictors for the development of new-onset hypertension.

We conclude that it is important to identify the prehypertensive subjects who will develop hypertension in the near future. We believe that markers are probably based on the physiological alterations prior to the onset of hypertension. Future work is needed to find easy to handle and cheap markers which allow screening of the prehypertensive subjects.

\section{The future of transient prehypertensive treatment}

Transient prehypertensive treatment in humans is not accepted yet. The recently published Trial of Preventing Hypertension (TROPHY) was the first clinical trial investigating this strategy (121). Although a risk reduction was demonstrated by this treatment strategy, the study received a lot of criticism. This was on one hand related to the general setting of the study including the recruitment of participants and end-point design (123). On the other 
hand colleagues were afraid that this might lead to a generalized and unselected treatment of the whole prehypertensive population.

A second yet unpublished study confirmed the findings of the TROPHY trial. The German multi-centre trial called PHARAO was presented at the ISH 2006 in Fukuoka and demonstrated the prehypertensive effectiveness of ACE-inhibiton in middle-aged.

Regarding the setting of prehypertensive treatment in humans a Dutch multi-centre trial has been created which aims to investigate this treatment strategy in a more selective manner including a clearer design.

The study is called Transient inhibition of the renin-angiotensin system (TIResiAS). In TIResiAS we chose a younger population (18-40 years of age) than the TROPHY trial (mean 48 years) since we expect in these subjects less cardiovascular damage which could result in a better blood pressure lowering and enhanced target organ protection (121). Furthermore we restricted the allowed cardiovascular risk factors for participation to less than 3 for the same reasons. In comparison nearly $50 \%$ of the participants of the TROPHY trial had 4 or more risk factors apparent (134). Another major difference in the trials is the choice of medication. While TROPHY chose an ARB, we chose an ACE-inhibitor, because studies in SHR revealed that part of the effects could be bradykinin mediated $(113,115)$. 


\section{SUMMARY}

Hypertension is an important worldwide public-health challenge because of its high incidence and associated risks of cardiovascular and kidney disease. It has been identified as the leading risk factor for mortality, and is ranked third as a cause of reduced disabilityadjusted life-years. Although antihypertensive therapy offers the potential to effectively reduce blood pressure and target organ damage the majority of hypertensives remains ineffectively treated. Therefore we must look for a feasible opportunity to improve the treatment regimen. An improved antihypertensive drug therapy offers one opportunity; a treatment which delays the onset of hypertension and the related target organ damage another.

This thesis describes transient prehypertensive treatment as a treatment strategy against hypertension and related target organ damage in the spontaneously hypertensive rat (SHR). The blood pressure lowering initiated by early treatment with the angiotensin II receptor type 1 blocker (ARB) losartan between weeks 4 to 8 of age is maintained for approximately 9 months. In adult rats the accumulation of deleterious hypertensive effects is reduced. Cardiac and renal damage are attenuated. No blood pressure lowering is apparent at higher age. However, the organ protective effects remain until 72 weeks of age which represents an advanced age for a SHR.

In contrast to the heart and kidneys, no major structural improvement is observed in blood vessels at advanced age. Mesenteric arteries demonstrate a hypertrophic remodelling despite early losartan treatment. However, functional alterations are apparent. The contraction of vascular SMC is stronger in old SHR after early losartan therapy. Secondly, the endothelium-mediated relaxation is improved after early losartan therapy as compared to placebo. This phenomenon is related to an intracellular change in distribution of eNOS in the endothelial cell.

Blood pressure independent effects seem to be involved in transient prehypertensive treatment in the SHR. One of these effects is the aging process. This process is accelerated in SHR, based on an enhanced cell-turnover. We demonstrated that this cell-turnover is not primarily related to blood pressure, but to the renin-angiotensin-aldosterone system (RAAS). Furthermore, early RAAS-antagonism as performed by transient prehypertensive treatment decelerates the cell-turnover and the aging process. The reduction of the aging process attenuates target organ damage in the kidney. A potential reason for the sustained 
deceleration of the aging process could be the resetting of the RAAS after transient prehypertensive treatment. We observed a reduction of angiotensin $I$ sensitivity after the early treatment. We furthermore investigated why blood pressure remains lowered after transient prehypertensive treatment. Therefore, we investigated the renal medullary microcirculation, as it is a dominant factor in long-term blood pressure regulation. We demonstrated that renal medullary pericytes are increased in number and are able to contract vasa recta in SHR. Therefore they potentially influence blood pressure level. We showed that transient prehypertensive treatment leads to a reduction of the number of pericytes. Moreover, we proved that the reduction of pericyte number is initiated by apoptosis of pericytes. Therefore, we conclude that the sustained blood pressure lowering after prehypertensive treatment is based on the reduction of pericyte number with concomitant alterations in medullary blood flow.

The number of prehypertensive humans is comparable to the number of hypertensives. Therefore. it is socioeconomically not feasible to perform transient prehypertensive treatment in all prehypertensive subjects. This is in particular relevant as we do not know when to perform best this kind of treatment at all in humans.

We hypothesized that the physiological characteristics of the development of hypertension are comparable in SHR and humans. Therefore we investigated urinary nitric oxide (NO) bioavailability and sodium reabsorption in prehypertensives. We were able to demonstrate that only prehypertensives prior to the new-onset of hypertension had reduced No bioavailability and sodium excretion. Therefore we conclude that, in the future, easy and cheap blood pressure and urine measurements could be used to determine which humans will soon develop hypertension and whether these people are to treat with the transient treatment strategy.

It remains the work for the future to establish transient prehypertensive treatment in humans and to validate potential predictors for the new-onset of hypertension. Beside these tasks we have to investigate whether ACE-inhibitors or renin-antagonists show the same or even better long-term effects as ARBs. Furthermore, the hypothesis has to be tested whether transient prehypertensive treatment is particularly working in subjects who in the near future will develop new-onset hypertension. This would imply that the development of hypertension is an active disease which can be actively treated. 
Finally, answering the question of the thesis, transient prehypertensive treatment has the potential to become an alternative treatment strategy against hypertension. 


\section{SAMENVATTING}

Hypertensie is door haar hoge incidentie alsmede wegens bijkomende risico's voor nierziekten en hart- en vaatziekten een belangrijk, wereldwijd probleem voor de volksgezondheid. $\mathrm{Er}$ is vastgesteld dat hypertensie de voornaamste risicofactor voor mortaliteit vormt en als derde op de ranglijst staat als oorzaak voor achteruitgang in levenskwaliteit. Hoewel antihypertensieve therapie de mogelijkheid biedt op doeltreffende wijze de bloeddruk terug te brengen en orgaanschade te beperken, wordt de meerderheid der hypertensieven ontoereikend behandeld. Daarom moeten wij uitkijken naar een haalbare gelegenheid de behandelkuur te verbeteren. Een verbeterde antihypertensieve geneesmiddeltherapie zou een mogelijkheid kunnen zijn. Een tweede mogelijkheid is medicamenteus het optreden van hypertensie en daarvan verwante orgaanschade te vertragen.

Dit proefschrift beschrijft een kortdurende prehypertensieve behandeling als een behandelstrategie tegen hypertensie en daaruit voortkomende orgaanschade in de spontaan hypertensieve rat (SHR). De bloeddrukdaling in gang gezet door een vroege behandeling met de angiotensine $\|$ receptor type 1 antagonist (ARB) losartan in de leeftijd van week 4 tot 8 wordt gedurende ongeveer 9 maanden gehandhaafd. In deze volwassen ratten wordt de opeenstapeling van schadelijke, hypertensieve effecten beperkt. Hart- en nierschade verminderen. Op hogere leeftijd wordt geen bloeddrukdaling meer gezien. De beschermende effecten op de organen blijven echter bestaan tot een leeftijd van 72 weken, hetgeen voor een SHR een gevorderde leeftijd betekent.

In tegenstelling tot het hart en de nieren wordt er geen grote structurele verbetering in de bloedvaten op gevorderde leeftijd waargenomen. Mesenteriale arteriën laten een hypertrofische remodellering zien ondanks de vroege behandeling met losartan. Er zijn echter duidelijk functieveranderingen. De samentrekking van de vasculaire gladde spier cellen (SMC) is sterker in oude SHR na vroege losartan-therapie. Ten tweede is de door het endothelium veroorzaakte ontspanning verbeterd na vroege losartan-therapie vergeleken met placebo. Dit verschijnsel hangt samen met een intracellulaire verandering in de verspreiding van eNOS in de endotheliale cel.

Bloeddrukonafhankelijke effecten lijken betrokken bij kortdurende prehypertensieve behandeling in de SHR. Een van deze effecten is het verouderingsproces. Dit proces wordt 
versneld in SHR, gebaseeerd op een vergrote celturnover. We hebben aangetoond dat deze celturnover niet primair gerelateerd is aan de bloeddruk, maar aan het renineangiotensine-aldosteron systeem (RAAS). Verder vertraagt vroeg RAAS-antagonisme zoals teweeg gebracht wordt door een kortdurende prehypertensieve behandeling de celturnover en het verouderingsproces. De vertraging van het verouderingsproces vermindert orgaanschade in de nier. Een mogelijke reden voor de blijvende vertraging van het verouderingsproces zou kunnen zijn de resetting van het RAAS na kortdurende prehypertensieve behandeling. We namen een afname van de angiotensine II gevoeligheid waar na de vroege behandeling.

Verder onderzochten wij waarom de bloeddruk laag blijtt na kortdurende prehypertensieve behandeling. Daarom keken wij naar de renale medullaire microcirculatie, aangezien deze een dominerende factor is in de bloeddrukregulatie op lange termijn. Wij toonden aan dat renale medullaire pericyten in aantal toenemen en in staat zijn de vasa recta in SHR samen te trekken. Daarom beïnvloeden zij mogelijk het bloeddrukniveau. Wij lieten zien dat kortdurende prehypertensieve behandeling leidt tot aan afname in pericytenaantal.

Bovendien bewezen wij dat de afname in pericytenaantal in gang wordt gezet door apoptose van de pericyten. Daarom concluderen wij dat de blijvende bloedddrukdaling na prehypertensieve behandeling is gebaseerd op de afname in pericytenaantal met daarmee gepaard gaande veranderingen in de medullaire bloedstroom.

Het aantal prehypertensieve mensen is vergelijkbaar met het aantal hypertensieven. Daarom is het socio-economisch niet haalbaar kortdurende prehypertensieve behandeling toe te passen in alle prehypertensieve personen. Dit is bijzonder relevant aangezien we niet weten wanneer dit soort behandeling het best aan mensen gegeven kan worden, als dit al mogelijk is.

We formuleerden de hypothese dat de fysiologische kenmerken van de ontwikkeling van hypertensie in SHR en mensen vergelijkbaar zijn. Daarom onderzochten wij in prehypertensieven de biologische beschikbaarheid van stifkstofoxide (NO) in de urine en natriumreabsortie. $W \mathrm{ij}$ waren in staat aan te tonen dat slechts prehypertensieven voorafgaande aan het optreden van hypertensie een afgenomen biologische beschikbaarheid van NO en natriumuitscheiding hadden. Daarom concluderen wij dat in de toekomst gemakkelijke en goedkope bloeddruk- en urinemetingen gebruikt kunnen worden om te bepalen welke mensen hypertensie zullen ontwikkelen en of deze mensen het best behandeld kunnen worden met de kortdurende behandelstrategie. 
Het is aan toekomstig onderzoek kortdurende prehypertensieve behandelingen in mensen verder te staven en mogelijke voorspellende factoren voor het optreden van hypertensie te bevestigen. Behalve deze taken dienen we te onderzoeken of ACE-remmers en renineantagonisten dezelfde of zelfs betere effecten op lange termijn laten zien als de ARB's. Verder moet de hypothese getest worden of een kortdurende prehypertensieve behandeling vooral werkt in mensen die op termijn hypertensie zullen ontwikkelen. Dit zou inhouden dat de ontwikkeling van hypertensie een actieve ziekte is die actief behandeld kan worden.

Tenslotte, in antwoord op de vraag van het proefschrift: kortdurende prehypertensieve behandelingen dragen de mogelijkheid tot verder onderzoek in zich en kunnen een alternatieve behandelstrategie tegen hypertensie worden. 


\section{REFERENCES}

1. (1999) 1999 World Health Organization--International Society of Hypertension Guidelines for the Management of Hypertension. Guidelines Sub-Committee. Blood Press Supp/1, 9-43

2. Meigs, J. B., D'Agostino, R. B., Sr., Wilson, P. W., Cupples, L. A., Nathan, D. M., and Singer, D. E. (1997) Risk variable clustering in the insulin resistance syndrome. The Framingham Offspring Study. Diabetes 46, 1594-1600

3. Zanchetti, A. (1997) The hypertensive patient with multiple risk factors: is treatment really so difficult? Am J Hypertens 10, 223S-229S

4. Stamler, J., Wentworth, D., and Neaton, J. D. (1986) Is relationship between serum cholesterol and risk of premature death from coronary heart disease continuous and graded? Findings in 356,222 primary screenees of the Multiple Risk Factor Intervention Trial (MRFIT). Jama 256, 2823-2828

5. Jackson, R. (2000) Updated New Zealand cardiovascular disease risk-benefit prediction guide. Bmj 320, 709-710

6. Kearney, P. M., Whelton, M., Reynolds, K., Muntner, P., Whelton, P. K., and He, J. (2005) Global burden of hypertension: analysis of worldwide data. Lancet 365, $217-$ 223

7. Yusuf, S., Reddy, S., Ounpuu, S., and Anand, S. (2001) Global burden of cardiovascular diseases: Part II: variations in cardiovascular disease by specific ethnic groups and geographic regions and prevention strategies. Circulation 104 2855-2864

8. Seedat, Y. K. (2000) Hypertension in developing nations in sub-Saharan Africa. $J$ Hum Hypertens 14, 739-747

9. Chobanian, A. V. (2006) Prehypertension revisited. Hypertension 48, 812-814

10. Vasan, R. S., Larson, M. G., Leip, E. P., Evans, J. C., O'Donnell, C. J., Kannel, W. B., and Levy, D. (2001) Impact of high-normal blood pressure on the risk of cardiovascular disease. N Engl J Med 345, 1291-1297

11. Wang, Y., and Wang, Q. J. (2004) The prevalence of prehypertension and hypertension among US adults according to the new joint national committee guidelines: new challenges of the old problem. Arch Intern Med 164, 2126-2134

12. Kjeldsen, S. E., Erdine, S., Farsang, C., Sleight, P., and Mancia, G. (2003) 1999 WHO/ISH Hypertension Guidelines--highlights \& ESH Update. Blood Press 12, 181 . 182

13. Cifkova, R., Erdine, S., Fagard, R., Farsang, C., Heagerty, A. M., Kiowski, W., Kjeldsen, S., Luscher, T., Mallion, J. M., Mancia, G., Poulter, N., Rahn, K. H., Rodicio, J. L., Ruilope, L. M., van Zwieten, P., Waeber, B., Williams, B., and Zanchetti, A. (2003) Practice guidelines for primary care physicians: 2003 ESH/ESC hypertension guidelines. J Hypertens 21, 1779-1786

14. PROGRESS (2001) Randomised trial of a perindopril-based blood-pressure-lowering regimen among 6,105 individuals with previous stroke or transient ischaemic attack. Lancet 358, 1033-1041

15. HOPE (2000) Effects of ramipril on cardiovascular and microvascular outcomes in people with diabetes mellitus: results of the HOPE study and MICRO-HOPE substudy. Heart Outcomes Prevention Evaluation Study Investigators. Lancet 355 , 253-259

16. Schrier, R. W., Estacio, R. O., Esler, A., and Mehler, P. (2002) Effects of aggressive blood pressure control in normotensive type 2 diabetic patients on albuminuria, retinopathy and strokes. Kidney Int 61, 1086-1097 
17. Willum-Hansen, T., Staessen, J. A., Torp-Pedersen, C., Rasmussen, S., Thijs, L., Ibsen, $H_{\text {. }}$, and Jeppesen, J. (2006) Prognostic value of aortic pulse wave velocity as index of arterial stiffness in the general population. Circulation 113, 664-670

18. Doll, R., Peto, R., Wheatley, K., Gray, R., and Sutherland, I. (1994) Mortality in relation to smoking: 40 years' observations on male British doctors. Bmj 309, 901 911

19. Primatesta, P., Falaschetti, E., Gupta, S., Marmot, M. G., and Poulter, N. R. (2001) Association between smoking and blood pressure: evidence from the health survey for England. Hypertension 37, 187-193

20. Omvik, P. (1996) How smoking affects blood pressure. Blood Press 5, 71-77

21. Wannamethee, S. G., and Shaper, A. G. (1996) Patterns of alcohol intake and risk of stroke in middle-aged British men. Stroke 27, 1033-1039

22. Stamler, J. (1991) Epidemiologic findings on body mass and blood pressure in adults. Ann Epidemiol 1, 347-362

23. Reid, C. M., Dart, A. M., Dewar, E. M., and Jennings, G. L. (1994) Interactions between the effects of exercise and weight loss on risk factors, cardiovascular haemodynamics and left ventricular structure in overweight subjects. J Hypertens 12 , 291-301

24. Puddey, I. B., Parker, M., Beilin, L. J., Vandongen, R., and Masarei, J. R. (1992) Effects of alcohol and caloric restrictions on blood pressure and serum lipids in overweight men. Hypertension 20,533-541

25. Whelton, P. K., Appel, L. J., Espeland, M. A., Applegate, W. B., Ettinger, W. H., Jr., Kostis, J. B., Kumanyika, S., Lacy, C. R., Johnson, K. C., Folmar, S., and Cutler, J. A. (1998) Sodium reduction and weight loss in the treatment of hypertension in older persons: a randomized controlled trial of nonpharmacologic interventions in the elderly (TONE). TONE Collaborative Research Group. Jama 279, 839-846

26. Sandvik, L., Erikssen, J., Thaulow, E., Erikssen, G., Mundal, R., and Rodahl, K. (1993) Physical fitness as a predictor of mortality among healthy, middle-aged Norwegian men. N Engl J Med 328, 533-537

27. Luft, F. C., and Weinberger, M. H. (1982) Sodium intake and essential hypertension. Hypertension 4, III14-19

28. Cutler, J. A., Follmann, D., and Allender, P. S. (1997) Randomized trials of sodium reduction: an overview. Am J Clin Nutr 65, 643S-651S

29. Beckmann, S. L., Os, I., Kjeldsen, S. E., Eide, I. K., Westheim, A. S., and Hjermann, I. (1995) Effect of dietary counselling on blood pressure and arterial plasma catecholamines in primary hypertension. Am J Hypertens 8, 704-711

30. Collins, R., Peto, R., MacMahon, S., Hebert, P., Fiebach, N. H., Eberlein, K. A., Godwin, J., Qizilbash, N., Taylor, J. O., and Hennekens, C. H. (1990) Blood pressure, stroke, and coronary heart disease. Part 2, Short-term reductions in blood pressure: overview of randomised drug trials in their epidemiological context. Lancet $335,827-838$

31. Collins, R., and MacMahon, S. (1994) Blood pressure, antihypertensive drug treatment and the risks of stroke and of coronary heart disease. Br Med Bull 50, 272298

32. Staessen, J. A., Gasowski, J., Wang, J. G., Thijs, L., Den Hond, E., Boissel, J. P., Coope, J., Ekbom, T., Gueyffier, F., Liu, L., Kerlikowske, K., Pocock, S., and Fagard, R. H. (2000) Risks of untreated and treated isolated systolic hypertension in the elderly: meta-analysis of outcome trials. Lancet $355,865-872$

33. Thijs, L., Fagard, R., Lijnen, P., Staessen, J., Van Hoof, R., and Amery, A. (1992) A meta-analysis of outcome trials in elderly hypertensives. J Hypertens 10, 1103-1109 
34. (1967) Effects of treatment on morbidity in hypertension. Results in patients with diastolic blood pressures averaging 115 through $129 \mathrm{~mm} \mathrm{Hg}$. Jama 202, 1028-1034

35. (1970) Effects of treatment on morbidity in hypertension. Il. Results in patients with diastolic blood pressure averaging 90 through $114 \mathrm{~mm} \mathrm{Hg}$. Jama 213, 1143-1152

36. Smith, W. C., Lee, A. J., Crombie, I. K., and Tunstall-Pedoe, H. (1990) Control of blood pressure in Scotland: the rule of halves. Bmj 300, 981-983

37. (2002) Major outcomes in high-risk hypertensive patients randomized to angiotensinconverting enzyme inhibitor or calcium channel blocker vs diuretic: The Antihypertensive and Lipid-Lowering Treatment to Prevent Heart Attack Trial (ALLHAT). Jama 288, 2981-2997

38. Schelleman, H., Klungel, O. H., Kromhout, D., de Boer, A., Stricker, B. H., and Verschuren, W. M. (2004) Prevalence and determinants of undertreatment of hypertension in the Netherlands. J Hum Hypertens 18, 317-324

39. Klungel, O. H., de Boer, A., Paes, A. H., Nagelkerke, N. J., Seidell, J. C., and Bakker, A. (2000) Estimating the prevalence of hypertension corrected for the effect of withinperson variability in blood pressure. Journal of clinical epidemiology 53, 1158-1163

40. Nesbitt, S. D., and Julius, S. (2000) Prehypertension: a possible target for antihypertensive medication. Curr Hypertens Rep 2, 356-361

41. Staessen, J. A., Wang, J. G., and Thijs, L. (2003) Cardiovascular prevention and blood pressure reduction: a quantitative overview updated until 1 March 2003. J Hypertens 21, 1055-1076

42. Goldblatt, H. (1947) The renal origin of hypertension. Physio/ Rev 27, 120-165

43. Goldblatt, H., Lynch, J., Hanzal, R, Summerville, WW. (1934) Studies on experimental hypertension. I. The production of persistent elevation of systolic blood pressure by means of renal ischemia. J Exp Med 59, 347-379

44. Moritz, A., and Oldt, M. (1937) Arteriolar sclerosis in hypertensive and nonhypertensive individuals. Am J Pathol 13, 679-728

45. Castleman, B., and Smithwick, R. (1943) The relation of vascular disease to the hypertensive state. Jama 12, 1256-1261

46. Sommers, S., Relman, R., and Smithwick, R. (1958) Histologic studies of kidney biopsy specimens from patients with hypertension. Am J Patho/ 34, 685-715

47. Perera, G. A. (1955) Hypertensive vascular disease; description and natural history. $J$ Chronic Dis 1, 33-42

48. Klag, M. J., Whelton, P. K., Randall, B. L., Neaton, J. D., Brancati, F. L., Ford, C. E., Shulman, N. B., and Stamler, J. (1996) Blood pressure and end-stage renal disease in men. N Engl J Med 334, 13-18

49. Guyton, A. C., Coleman, T. G., Cowley, A. V., Jr., Scheel, K. W., Manning, R. D., Jr. and Norman, R. A., Jr. (1972) Arterial pressure regulation. Overriding dominance of the kidneys in long-term regulation and in hypertension. Am.J.Med. 52, 584-594

50. Borst J.G.G., B. d. G., A. (1963) Hypertension explained by Starling's law of circulatory homeostasis. Lancet 1, 677-680

51. Safar, M. E., London, G. M., and Plante, G. E. (2004) Arterial stiffness and kidney function. Hypertension 43, 163-168

52. Hall, J. E., Brands, M. W., and Henegar, J. R. (1999) Mechanisms of hypertension and kidney disease in obesity. Ann.N.Y.Acad.Sci. 892, 91-107

53. Rettig, R., and Grisk, $O$. (2005) The kidney as a determinant of genetic hypertension: evidence from renal transplantation studies. Hypertension 46, 463-468

54. Bianchi, G., Fox, U., Di Francesco, G. F., Giovanetti, A. M., and Pagetti, D. (1974) Blood pressure changes produced by kidney cross-transplantation between 
spontaneously hypertensive rats and normotensive rats. Clin Sci Mol Med 47, 435448

55. Rettig, R., Stauss, H., Folberth, C., Ganten, D., Waldherr, B., and Unger, T. (1989) Hypertension transmitted by kidneys from stroke-prone spontaneously hypertensive rats. Am J Physiol 257, F197-203

56. Curtis, J. J., Luke, R. G., Dustan, H. P., Kashgarian, M., Whelchel, J. D., Jones, P., and Diethelm, A. G. (1983) Remission of essential hypertension after renal transplantation. N Engl J Med 309, 1009-1015

57. Guidi, E., Bianchi, G., Rivolta, E., Ponticelli, C., Quarto di Palo, F., Minetti, L., and Polli, E. (1985) Hypertension in man with a kidney transplant: role of familial versus other factors. Nephron 41, 14-21

58. Guidi, E., Menghetti, D., Milani, S., Montagnino, G., Palazzi, P., and Bianchi, G. (1996) Hypertension may be transplanted with the kidney in humans: a long-term historical prospective follow-up of recipients grafted with kidneys coming from donors with or without hypertension in their families. J Am Soc Nephrol 7, 1131-1138

59. Warnock, D. G. (1998) Liddle syndrome: an autosomal dominant form of human hypertension. Kidney Int 53, 18-24

60. Folkow, B., Hallback, M., Lundgren, Y., and Weiss, L. (1971) Renal vascular resistance in spontaneously hypertensive rats. Acta Physiol Scand 83, 96-105

61. Folkow, B., Gothberg, G., Lundin, S., and Ricksten, S. E. (1977) Structural renal vascular changes in renal hypertensive rats (RHR). Acta Physiol Scand 101, 254-256

62. Folkow, B., Gothberg, G., Lundin, S., and Ricksten, S. E. (1977) Structural "resetting" of the renal vascular bed in spontaneously hypertensive rats (SHR). Acta Physiol Scand 100, 270-272

63. Ledingham, J. M., and Laverty, R. (1998) Renal afferent arteriolar structure in the genetically hypertensive $(\mathrm{GH})$ rat and the ability of losartan and enalapril to cause structural remodelling. J.Hypertens. 16, 1945-1952

64. Skov, K., Mulvany, M. J., and Korsgaard, N. (1992) Morphology of renal afferent arterioles in spontaneously hypertensive rats. Hypertension 20, 821-827

65. Smeda, J. S., Lee, R. M., and Forrest, J. B. (1988) Structural and reactivity alterations of the renal vasculature of spontaneously hypertensive rats prior to and during established hypertension. Circ Res 63, 518-533

66. Skov, K., Nyengaard, J. R., Korsgaard, N., and Mulvany, M. J. (1994) Number and size of renal glomeruli in spontaneously hypertensive rats. J.Hypertens. 12, 13731376

67. Skov, K., Nyengaard, J. R., Patwardan, A., and Mulvany, M. J. (1999) Large juxtamedullary glomeruli and afferent arterioles in healthy primates. Kidney Int. 55, 1462-1469

68. Keller, G., Zimmer, G., Mall, G., Ritz, E., and Amann, K. (2003) Nephron number in patients with primary hypertension. N Engl J Med 348, 101-108

69. de Leeuw, P. W., Hoogma, R. P., van Soest, G. A., van Es, P. N., and Birkenhager, W. H. (1983) Physiological effects of short-term treatment with enalapril in hypertensive patients. J Hypertens Supp/ 1, 87-91

70. van Hooft, I. M., Grobbee, D. E., Derkx, F. H., de Leeuw, P. W., Schalekamp, M. A., and Hofman, A. (1991) Renal hemodynamics and the renin-angiotensin-aldosterone system in normotensive subjects with hypertensive and normotensive parents. $N$ Engl J Med 324, 1305-1311

71. van Hooft, I. M., Grobbee, D. E., Waal-Manning, H. J., and Hofman, A. (1993) Hemodynamic characteristics of the early phase of primary hypertension. The Dutch Hypertension and Offspring Study. Circulation 87, 1100-1106 
72. Cowley, A. W., Jr., Mattson, D. L., Lu, S., and Roman, R. J. (1995) The renal medulla and hypertension. Hypertension 25, 663-673

73. Lu, S., Mattson, D. L., and Cowley, A. W., Jr. (1994) Renal medullary captopril delivery lowers blood pressure in spontaneously hypertensive rats. Hypertension 23, 337-345

74. Miyata, N., and Cowley, A. W., Jr. (1999) Renal intramedullary infusion of L-arginine prevents reduction of medullary blood flow and hypertension in Dahl salt-sensitive rats. Hypertension 33, 446-450

75. Mattson, D. L., Lu, S., Nakanishi, K., Papanek, P. E., and Cowley, A. W., Jr. (1994) Effect of chronic renal medullary nitric oxide inhibition on blood pressure. Am J Physiol 266, H1918-1926

76. Rodriguez-Iturbe, B., Pons, H., Herrera-Acosta, J., and Johnson, R. J. (2001) Role of immunocompetent cells in nonimmune renal diseases. Kidney Int. 59, 1626-1640

77. Johnson, R. J., Herrera-Acosta, J., Schreiner, G. F., and Rodriguez-Iturbe, B. (2002) Subtle acquired renal injury as a mechanism of salt-sensitive hypertension. N Engl $J$ Med 346, 913-923

78. Kang, D. H., Kim, Y. G., Andoh, T. F., Gordon, K. L., Suga, S., Mazzali, M. Jefferson, J. A., Hughes, J., Bennett, W., Schreiner, G. F., and Johnson, R. J. (2001) Post-cyclosporine-mediated hypertension and nephropathy: amelioration by vascular endothelial growth factor. Am.J.Physiol Renal Physiol 280, F727-F736

79. Myers, B. D., Deen, W. M., and Brenner, B. M. (1975) Effects of norepinephrine and angiotensin II on the determinants of glomerular ultrafiltration and proximal tubule fluid reabsorption in the rat. Circ Res 37, 101-110

80. Zhong, Z., Arteel, G. E., Connor, H. D., Yin, M., Frankenberg, M. V., Stachlewitz, R. F., Raleigh, J. A., Mason, R. P., and Thurman, R. G. (1998) Cyclosporin A increases hypoxia and free radical production in rat kidneys: prevention by dietary glycine. $\mathrm{Am}$ J Physiol 275, F595-604

81. Johnson, R. J., Rodriguez-Iturbe, B., Schreiner, G. F., and Herrera-Acosta, J. (2002) Hypertension: a microvascular and tubulointerstitial disease. $J$ Hypertens 20 Suppl 3, S1-7

82. Roman, R. J., and Kaldunski, M. L. (1988) Renal cortical and papillary blood flow in spontaneously hypertensive rats. Hypertension 11, 657-663

83. Thomas, M. C., Tikellis, C., Burns, W. M., Bialkowski, K., Cao, Z., Coughlan, M. T., Jandeleit-Dahm, K., Cooper, M. E., and Forbes, J. M. (2005) Interactions between Renin Angiotensin System and Advanced Glycation in the Kidney. J Am Soc Nephrol 16, 2976-2984

84. Harrap, S. B., Mirakian, C., Datodi, S. R., and Lever, A. F. (1994) Blood pressure and lifespan following brief ACE inhibitor treatment in young spontaneously hypertensive rats. Clin Exp Pharmacol Physiol 21, 125-127

85. Evenwel, R. T., Kasbergen, C. M., and Struyker-Boudier, H. A. (1983) Central and regional hemodynamics and plasma volume distribution during the development of spontaneous hypertension in rats. Clin Exp Hypertens A 5, 1511-1536

86. Chrysant, S. G., Mandal, A. K., and Nordquist, J. A. (1980) Renal functional and organic changes induced by salt and prostaglandin inhibition in spontaneously hypertensive rats. Nephron 25, 151-155

87. Pettinger, W. A., Sanchez, A., Saavedra, J., Haywood, J. R., Gandler, T., and Rodes, T. (1982) Altered renal alpha 2-adrenergic receptor regulation in genetically hypertensive rats. Hypertension 4, 188-192

88. Winternitz, S. R., and Oparil, S. (1982) Sodium-neural interactions in the development of spontaneous hypertension. Clin Exp Hypertens A 4, 751-760 
89. Toal, C. B., and Leenen, F. H. (1983) Dietary sodium restriction and development of hypertension in spontaneously hypertensive rats. Am J Physiol 245, H1081-1084

90. Toal, C. B., and Leenen, F. H. (1987) Dietary sodium restriction, blood pressure and sympathetic activity in spontaneously hypertensive rats. J.Hypertens. 5, 107-113

91. Wright, G. L., Mc Murtry, J. P., and Wexler, B. C. (1981) Food restriction reduces the blood pressure of the spontaneously hypertensive rat. Life Sci 28, 1253-1259

92. Young, J. B., Mullen, D., and Landsberg, L. (1978) Caloric restriction lowers blood pressure in the spontaneously hypertensive rat. Metabolism 27, 1711-1714

93. Toal, C. B., and Leenen, F. H. (1983) Body fluid volumes during development of hypertension in the spontaneously hypertensive rat. J.Hypertens. 1, 345-350

94. Mullins, M. M. (1983) Body fluid volumes in prehypertensive spontaneously hypertensive rats. Am.J.Physiol 244, H652-H655

95. Mullins, M. M., Kleinman, L. I., Russell, P. T., and Srivastava, L. S. (1982) Plasma aldosterone concentrations in neonatal spontaneously hypertensive rats. Life Sci 31, 2751-2755

96. Cheng, H. F., Wang, J. L., Vinson, G. P., and Harris, R. C. (1998) Young SHR express increased type 1 angiotensin II receptors in renal proximal tubule. Am J Physiol 274, F10-17

97. Brannstrom, K., Morsing, P., and Arendshorst, W. J. (1996) Exaggerated tubuloglomerular feedback activity in genetic hypertension is mediated by ANG II and AT1 receptors. Am J Physiol 270, F749-755

98. Brannstrom, K., Morsing, P., and Arendshorst, W. J. (1999) Candesartan normalizes exaggerated tubuloglomerular feedback activity in young spontaneously hypertensive rats. J Am Soc Nephrol 10 Suppl 11, S213-219

99. Brannstrom, K., and Arendshorst, W. J. (1999) Thromboxane A2 contributes to the enhanced tubuloglomerular feedback activity in young SHR. Am J Physiol 276, F758766

100. Grone, H. J., Grippo, R. S., Arendshorst, W. J., and Dunn, M. J. (1986) Role of thromboxane in control of arterial pressure and renal function in young spontaneously hypertensive rats. Am J Physiol 250, F488-496

101. Sacerdoti, D., Escalante, B., Abraham, N. G., McGiff, J. C., Levere, R. D., and Schwartzman, M. L. (1989) Treatment with tin prevents the development of hypertension in spontaneously hypertensive rats. Science $\mathbf{2 4 3}, 388-390$

102. Croft, K. D., McGiff, J. C., Sanchez-Mendoza, A., and Carroll, M. A. (2000) Angiotensin II releases 20-HETE from rat renal microvessels. Am.J.Physiol Renal Physiol 279, F544-F551

103. Berecek, K. H., Nagahama, S., and Oparil, S. (1984) Effect of central administration of MK-422 (the diacid form of enalapril) on the development of hypertension in the spontaneously hypertensive rat. J Hypertens Supp/ 2, S63-66

104. Bergstrom, G., Johansson, I., Wickman, A., Gan, L., and Thorup, C. (2002) Brief losartan treatment in young spontaneously hypertensive rats abates long-term blood pressure elevation by effects on renal vascular structure. J Hypertens 20, 1413-1421

105. Nakaya, H., Sasamura, H., Hayashi, M., and Saruta, T. (2001) Temporary treatment of prepubescent rats with angiotensin inhibitors suppresses the development of hypertensive nephrosclerosis. J Am Soc Nephrol 12, 659-666

106. Martens, J. R., Reaves, P. Y., Lu, D., Katovich, M. J., Berecek, K. H., Bishop, S. P., Raizada, M. K., and Gelband, C. H. (1998) Prevention of renovascular and cardiac pathophysiological changes in hypertension by angiotensin 11 type 1 receptor antisense gene therapy. Proc Natl Acad Sci U S A 95, 2664-2669 
107. Martens, J. R., Reaves, P. Y., Lu, D., Katovich, M. J., Berecek, K. H., Bishop, S. P., Raizada, M. K., and Gelband, C. H. (2004) Prevention of renovascular and cardiac pathophysiological changes in hypertension by angiotensin II type 1 receptor antisense gene therapy. Proc Natl Acad SciU S A

108. Regan, C. P., Bishop, S. P., and Berecek, K. H. (1997) Early, short-term treatment with captopril permanently attenuates cardiovascular changes in spontaneously hypertensive rats. Clin Exp Hypertens 19, 1161-1177

109. Wu, J. N., Edwards, D., and Berecek, K. H. (1994) Changes in renal angiotensin II receptors in spontaneously hypertensive rats by early treatment with the angiotensinconverting enzyme inhibitor captopril. Hypertension 23, 819-822

110. Harrap, S. B., and Doyle, A. E. (1986) Renal haemodynamics and total body sodium in immature spontaneously hypertensive and Wistar-Kyoto rats. $J$ Hypertens Supp/4, S249-252

111. Harrap, S. B., Van der Merwe, W. M., Griffin, S. A., Macpherson, F., and Lever, A. F. (1990) Brief angiotensin converting enzyme inhibitor treatment in young spontaneously hypertensive rats reduces blood pressure long-term. Hypertension 16, 603-614

112. Jones, E. F., Harrap, S. B., Calafiore, P., and Tonkin, A. M. (1992) Development and validation of echocardiographic methods for estimating left ventricular mass in rats. Clin Exp Pharmacol Physiol 19, 361-364

113. O'Sullivan, J. B., and Harrap, S. B. (1995) Resetting blood pressure in spontaneously hypertensive rats. The role of bradykinin. Hypertension 25, 162-165

114. O'Sullivan, J. B., Bertram, J. F., and Harrap, S. B. (1995) Renal medulla and bradykinin during the development of hypertension in SHR. Clin Exp Pharmacol Physiol 22, 463-465

115. O'Sullivan, J. B., and Harrap, S. B. (1999) Long-term effects of angiotensinconverting enzyme inhibition on renal medullary neutral lipid in spontaneously hypertensive rats. Hypertension 33, 1214-1217

116. Kost, C. K., Jr., Li, P., and Jackson, E. K. (1995) Blood pressure after captopril withdrawal from spontaneously hypertensive rats. Hypertension 25, 82-87

117. Rizzoni, D., Castellano, M., Porteri, E., Bettoni, G., Muiesan, M. L., and Agabiti Rosei, E. (1994) Delayed development of hypertension after short-term nitrendipine treatment. Hypertension 24, 131-139

118. Christensen, K. L., Jespersen, L. T., and Mulvany, M. J. (1989) Development of blood pressure in spontaneously hypertensive rats after withdrawal of long-term treatment related to vascular structure. $J$ Hypertens 7, 83-90

119. Li, J. S., and Schiffrin, E. L. (1995) Chronic endothelin receptor antagonist treatment of young spontaneously hypertensive rats. J Hypertens 13, 647-652

120. Pollock, D. M., and Morsing, P. (1999) Combined treatment with ibuprofen and the AT1 receptor antagonist candesartan in young spontaneously hypertensive rats. $J$ Am Soc Nephrol 10 Suppl 11, S116-119

121. Julius, S., Nesbitt, S. D., Egan, B. M., Weber, M. A., Michelson, E. L., Kaciroti, N., Black, H. R., Grimm, R. H., Jr., Messerli, F. H., Oparil, S., and Schork, M. A. (2006) Feasibility of Treating Prehypertension with an Angiotensin-Receptor Blocker. N Engl $J$ Med 354, 1685-1697

122. Meltzer, J.I. (2006) A specialist in clinical hypertension critiques the TROPHY trial. Am J Hypertens 19, 1098-1100

123. Baumann, M., and van den Born, B. J. (2006) Treating prehypertension. N Engl J Med 355, 416-418; author reply 
124. Kai, H. Kuwahara, F., Tokuda, K., and Imaizumi, T. (2005) Diastolic dysfunction in hypertensive hearts: roles of perivascular inflammation and reactive myocardial fibrosis. Hypertens Res 28, 483-490

125 Weber. K. T., and Brilla, C. G. (1991) Pathological hypertrophy and cardiac interstitium. Fibrosis and renin-angiotensin-aldosterone system. Circulation 83, 18491865

126. Paradis, P., Dali-Youcef, N., Paradis, F. W., Thibault, G., and Nemer, M. (2000) Overexpression of angiotensin II type I receptor in cardiomyocytes induces cardiac hypertrophy and remodeling. Proc Natl Acad Sci U S A 97, 931-936

127. Crabos, M., Roth, M., Hahn, A. W., and Erne, P. (1994) Characterization of angiotensin $\|$ receptors in cultured adult rat cardiac fibroblasts. Coupling to signaling systems and gene expression. J Clin Invest 93, 2372-2378

128. Fischer, T. A., Ungureanu-Longrois, D., Singh, K., de Zengotita, J., DeUgarte, D., Alali, A., Gadbut, A. P., Lee, M. A., Balligand, J. L., Kifor, I., Smith, T. W., and Kelly, R. A. (1997) Regulation of bFGF expression and ANG II secretion in cardiac myocytes and microvascular endothelial cells. Am J Physiol 272, H958-968

129. Varagic, J., and Frohlich, E. D. (2002) Local cardiac renin-angiotensin system: hypertension and cardiac failure. J Mol Cell Cardiol 34, 1435-1442

130. Brilla, C. G., Janicki, J. S., and Weber, K. T. (1991) Cardioreparative effects of lisinopril in rats with genetic hypertension and left ventricular hypertrophy. Circulation 83, 1771-1779

131. Nishikawa, K. (1998) Angiotensin AT1 receptor antagonism and protection against cardiovascular end-organ damage. J Hum Hypertens 12, 301-309

132. Yokoyama, H., Averill, D. B., Brosnihan, K. B., Smith, R. D., Schiffrin, E. L., and Ferrario, C. M. (2005) Role of blood pressure reduction in prevention of cardiac and vascular hypertrophy. Am J Hypertens 18, 922-929

133. Reed, S. D., Radeva, J. I., Weinfurt, K. P., McMurray, J. J., Pfeffer, M. A., Velazquez, E. J., Allsbrook, J. S., Masselink, L. E., Sellers, M. A., Califf, R. M., and Schulman, K. A. (2005) Resource use, costs, and quality of life among patients in the multinational Valsartan in Acute Myocardial Infarction Trial (VALIANT). Am Heart J 150, 323-329

134. Boyd, C. M., Darer, J., Boult, C., Fried, L. P., Boult, L., and Wu, A. W. (2005) Clinical practice guidelines and quality of care for older patients with multiple comorbid diseases: implications for pay for performance. Jama 294, 716-724

135. Janssen, B. J., Struijker Boudier, H. A., and Smits, J. F. (1994) Role of afferent renal nerves in renal adaptation to sodium restriction in uninephrectomized rats. Acta Physiol Scand 151, 395-402

136. Anderson, N. H., Devlin, A. M., Graham, D., Morton, J. J., Hamilton, C. A., Reid, J. L., Schork, N. J., and Dominiczak, A. F. (1999) Telemetry for cardiovascular monitoring in a pharmacological study: new approaches to data analysis. Hypertension 33, 248255

137. Janssen, B. J., De Celle, T., Debets, J. J., Brouns, A. E., Callahan, M. F., and Smith, T. L. (2004) Effects of anesthetics on systemic hemodynamics in mice. Am J Physiol Heart Circ Physiol 287, H1618-1624

138. Yu, Y., Ohmori, K., Kondo, I., Yao, L., Noma, T., Tsuji, T., Mizushige, K., and Kohno, M. (2002) Correlation of functional and structural alterations of the coronary arterioles during development of type II diabetes mellitus in rats. Cardiovasc Res 56, 303-311

139. Rakusan, K., Cicutti, N., Kazda, S., and Turek, Z. (1994) Effect of nifedipine on coronary capillary geometry in normotensive and hypertensive rats. Hypertension 24, 205-211 
140. Cingolani, O. H., Yang, X. P., Cavasin, M. A., and Carretero, O. A. (2003) Increased systolic performance with diastolic dysfunction in adult spontaneously hypertensive rats. Hypertension 41, 249-254

141. Schellings, M. W., Baumann, M., van Leeuwen, R. E., Duisters, R. F., Janssen, S. H., Schroen, B., Peutz-Kootstra, C. J., Heymans, S., and Pinto, Y. M. (2006) Imatinib attenuates end-organ damage in hypertensive homozygous TGR(mRen2)27 rats. Hypertension 47, 467-474

142. Ofstad, J., and Iversen, B. M. (2005) Glomerular and tubular damage in normotensive and hypertensive rats. Am J Physiol Renal Physiol 288, F665-672

143. Slama, M., Ahn, J., Varagic, J., Susic, D., and Frohlich, E. D. (2004) Long-term left ventricular echocardiographic follow-up of SHR and WKY rats: effects of hypertension and age. Am J Physiol Heart Circ Physiol 286, H181-185

144. Luchner, A., Hengstenberg, C., Lowel, H., Riegger, G. A., Schunkert, H., and Holmer, $S$. (2005) Effect of compensated renal dysfunction on approved heart failure markers: direct comparison of brain natriuretic peptide (BNP) and N-terminal pro-BNP. Hypertension 46, 118-123

145. Jalil, J. E., Doering, C. W., Janicki, J. S., Pick, R., Shroff, S. G., and Weber, K. T. (1989) Fibrillar collagen and myocardial stiffness in the intact hypertrophied rat left ventricle. Circ Res 64, 1041-1050

146. Conrad, C. H., Brooks, W. W., Hayes, J. A., Sen, S., Robinson, K. G., and Bing, O. H. (1995) Myocardial fibrosis and stiffness with hypertrophy and heart failure in the spontaneously hypertensive rat. Circulation 91, 161-170

147. Struijker Boudier, H. A., le Noble, J. L., Messing, M. W., Huijberts, M. S., Le Noble, F. A., and van Essen, H. (1992) The microcirculation and hypertension. J.Hypertens.Supp/10, S147-S156

148. Houben, A. J., Beljaars, J. H., Hofstra, L., Kroon, A. A., and De Leeuw, P. W. (2003) Microvascular abnormalities in chronic heart failure: a cross-sectional analysis. Microcirculation 10, 471-478

149. Kacimi, R., and Gerdes, A. M. (2003) Alterations in G protein and MAP kinase signaling pathways during cardiac remodeling in hypertension and heart failure. Hypertension 41, 968-977

150. Lindpaintner, K., and Ganten, D. (1991) The cardiac renin-angiotensin system. An appraisal of present experimental and clinical evidence. Circ Res 68, 905-921

151. Raizada, M. K., and Der Sarkissian, S. (2006) Potential of gene therapy strategy for the treatment of hypertension. Hypertension 47, 6-9

152. Folkow, B. (1982) Physiological aspects of primary hypertension. Physiol Rev 62, 347-504

153. Neves, M. F., Virdis, A., and Schiffrin, E. L. (2003) Resistance artery mechanics and composition in angiotensin II-infused rats: effects of aldosterone antagonism. $J$ Hypertens 21, 189-198

154. Virdis, A., Neves, M. F., Amiri, F., Viel, E., Touyz, R. M., and Schiffrin, E. L. (2002) Spironolactone improves angiotensin-induced vascular changes and oxidative stress. Hypertension 40, 504-510

155. Morton, J. J., Beattie, E. C., and MacPherson, F. (1992) Angiotensin II receptor antagonist losartan has persistent effects on blood pressure in the young spontaneously hypertensive rat: lack of relation to vascular structure. J Vasc Res 29, 264-269

156. Rizzoni, D., Porteri, E., Piccoli, A., Castellano, M., Bettoni, G., Muiesan, M. L., Pasini, G., Guelfi, D., Mulvany, M. J., and Agabiti, R. E. (1998) Effects of losartan and enalapril on small artery structure in hypertensive rats. Hypertension 32, 305-310 
157. Skov, K., Fenger-Gron, J., and Mulvany, M. J. (1996) Effects of an angiotensinconverting enzyme inhibitor, a calcium antagonist, and an endothelin receptor antagonist on renal afferent arteriolar structure. Hypertension 28, 464-471

158. van Zandvoort, M., Engels, W., Douma, K., Beckers, L., Oude Egbrink, M., Daemen, M. and Slaaf, D. W. (2004) Two-photon microscopy for imaging of the (atherosclerotic) vascular wall: a proof of concept study. J Vasc Res 41, 54-63

159. Endemann, D., Touyz, R. M., Li, J. S., Deng, L. Y., and Schiffrin, E. L. (1999) Altered angiotensin II-induced small artery contraction during the development of hypertension in spontaneously hypertensive rats. Am J Hypertens 12, 716-723

160. Touyz, R. M., Endemann, D., He, G., Li, J. S., and Schiffrin, E. L. (1999) Role of AT2 receptors in angiotensin II-stimulated contraction of small mesenteric arteries in young SHR. Hypertension 33, 366-372

161. Baumann M., S. J. F. M., Struijker Boudier H.A.J. (2005) Long-term echocardiographic follow up of brief and early losartan treated SHR reveals sustained cardiac protection. Hypertension 46, 896

162. Demirci, B., McKeown, P. P., and Bayraktutan, U. (2005) Blockade of angiotensin II provides additional benefits in hypertension- and ageing-related cardiac and vascular dysfunctions beyond its blood pressure-lowering effects. J Hypertens 23, 2219-2227

163. Gillies, L. K., Lu, M., Wang, H., and Lee, R. M. (1997) AT1 receptor antagonist treatment caused persistent arterial functional changes in young spontaneously hypertensive rats. Hypertension 30, 1471-1478

164. Touyz, R. M., and Schiffrin, E. L. (2001) Increased generation of superoxide by angiotensin II in smooth muscle cells from resistance arteries of hypertensive patients: role of phospholipase D-dependent NAD $(P) H$ oxidase-sensitive pathways. $J$ Hypertens 19, 1245-1254

165. Rajagopalan, S., Kurz, S., Munzel, T., Tarpey, M., Freeman, B. A., Griendling, K. K., and Harrison, D. G. (1996) Angiotensin II-mediated hypertension in the rat increases vascular superoxide production via membrane NADH/NADPH oxidase activation. Contribution to alterations of vasomotor tone. J Clin Invest 97, 1916-1923

166. Landmesser, U., Dikalov, S., Price, S. R., McCann, L., Fukai, T., Holland, S. M., Mitch, W. E., and Harrison, D. G. (2003) Oxidation of tetrahydrobiopterin leads to uncoupling of endothelial cell nitric oxide synthase in hypertension. J Clin Invest 111, 1201-1209

167. Wilcox, C. S. (2005) Oxidative stress and nitric oxide deficiency in the kidney: a critical link to hypertension? Am J Physiol Regul Integr Comp Physiol 289, R913-935

168. Cheng, C., van Haperen, R., de Waard, M., van Damme, L. C., Tempel, D., Hanemaaijer, L., van Cappellen, G. W., Bos, J., Slager, C. J., Duncker, D. J., van der Steen, A. F., de Crom, R., and Krams, R. (2005) Shear stress affects the intracellular distribution of eNOS: direct demonstration by a novel in vivo technique. Blood 106 , 3691-3698

169. Zhang, Q., Church, J. E., Jagnandan, D., Catravas, J. D., Sessa, W. C., and Fulton, D. (2006) Functional Relevance of Golgi- and Plasma Membrane-Localized Endothelial NO Synthase in Reconstituted Endothelial Cells. Arterioscler Thromb Vasc Biol 26, 1015-1021

170. Forstermann, U., and Munzel, T. (2006) Endothelial nitric oxide synthase in vascular disease: from marvel to menace. Circulation 113, 1708-1714

171. Staessen, J. A., Fagard, R., Thijs, L., Celis, H., Arabidze, G. G., Birkenhager, W. H., Bulpitt, C. J., de Leeuw, P. W., Dollery, C. T., Fletcher, A. E., Forette, F., Leonetti, G., Nachev, C., O'Brien, E. T., Rosenfeld, J., Rodicio, J. L., Tuomilehto, J., and Zanchetti, A. (1997) Randomised double-blind comparison of placebo and active 
treatment for older patients with isolated systolic hypertension. The Systolic Hypertension in Europe (Syst-Eur) Trial Investigators. Lancet 350, 757-764

172. Aviv, A., and Zahorodny, W. (2001) Telomeres: the time factor in essential hypertension. Curr Hypertens Rep 3, 33-35

173. Hamet, P., Thorin-Trescases, N., Moreau, P., Dumas, P., Tea, B. S., deBlois, D., Kren, V., Pravenec, M., Kunes, J., Sun, Y., and Tremblay, J. (2001) Workshop: excess growth and apoptosis: is hypertension a case of accelerated aging of cardiovascular cells? Hypertension 37, 760-766

174. Epel, E. S., Lin, J., Wilhelm, F. H., Wolkowitz, O. M., Cawthon, R., Adler, N. E., Dolbier, C., Mendes, W. B., and Blackburn, E. H. (2005) Cell aging in relation to stress arousal and cardiovascular disease risk factors. Psychoneuroendocrinology

175. Jeanclos, E., Schork, N. J., Kyvik, K. O., Kimura, M., Skurnick, J. H., and Aviv, A. (2000) Telomere length inversely correlates with pulse pressure and is highly familial. Hypertension 36, 195-200

176. Thorin-Trescases, N., deBlois, D., and Hamet, P. (2001) Evidence of an altered in vivo vascular cell turnover in spontaneously hypertensive rats and its modulation by long-term antihypertensive treatment. J Cardiovasc Pharmacol 38, 764-774

177. Hamet, P., Richard, L., Dam, T. V., Teiger, E., Orlov, S. N., Gaboury, L., Gossard, F., and Tremblay, J. (1995) Apoptosis in target organs of hypertension. Hypertension 26, 642-648

178. Nadal, J. A., Scicli, G. M., Carbini, L. A., and Scicli, A. G. (2002) Angiotensin II stimulates migration of retinal microvascular pericytes: involvement of TGF-beta and PDGF-BB. Am.J.Physiol Heart Circ.Physiol 282, H739-H748

179. Mezzano, S. A., Ruiz-Ortega, M., and Egido, J. (2001) Angiotensin If and renal fibrosis. Hypertension 38, 635-638

180. Choi, J. H., Yoo, K. H., Cheon, H. W., Kim, K. B., Hong, Y. S., Lee, J. W., Kim, S. K., and $\mathrm{Kim}, \mathrm{C} . \mathrm{H}$. (2002) Angiotensin converting enzyme inhibition decreases cell turnover in the neonatal rat heart. Pediatr Res 52, 325-332

181. Dickhout, J. G., and Lee, R. M. (1999) Apoptosis in the muscular arteries from young spontaneously hypertensive rats. J Hypertens 17, 1413-1419

182. Border, W. A., Noble, N. A., Yamamoto, T., Harper, J. R., Yamaguchi, Y., Pierschbacher, M. D., and Ruoslahti, E. (1992) Natural inhibitor of transforming growth factor-beta protects against scarring in experimental kidney disease. Nature $360,361-364$

183. Tanner, F. C., Greutert, H., Barandier, C., Frischknecht, K., and Luscher, T. F. (2003) Different cell cycle regulation of vascular smooth muscle in genetic hypertension. Hypertension 42, 184-188

184. Nozato, T., Ito, H., Tamamori, M., Adachi, S., Abe, S., Marumo, F., and Hiroe, M. (2000) $\mathrm{G} 1$ cyclins are involved in the mechanism of cardiac myocyte hypertrophy induced by angiotensin II. Jpn Circ J 64, 595-601

185. Diep, Q. N., El Mabrouk, M., Touyz, R. M., and Schiffrin, E. L. (2001) Expression of cell cycle proteins in blood vessels of angiotensin Il-infused rats: role of $A T(1)$ receptors. Hypertension 37, 604-608

186. Kintscher, U., Bruemmer, D., Blaschke, F., Unger, T., and Law, R. E. (2003) p38 MAP kinase negatively regulates angiotensin II-mediated effects on cell cycle molecules in human coronary smooth muscle cells. Biochem Biophys Res Commun 305, 552-556

187. Basso, N., Paglia, N., Stella, I., de Cavanagh, E. M., Ferder, L., del Rosario Lores Arnaiz, M., and Inserra, F. (2005) Protective effect of the inhibition of the reninangiotensin system on aging. Regul Pept 128, 247-252 
188. Chatziantoniou, C., and Arendshorst, W. J. (1993) Angiotensin receptor sites in renal vasculature of rats developing genetic hypertension. Am J Physiol 265, F853-862

189. Henrich, W. L., and Levi, M. (1991) Ontogeny of renal renin release in spontaneously hypertensive rat and Wistar-Kyoto rat. Am J Physiol 260, F530-535

190. Baumann, M., Janssen, B. J., Hermans, J. R., Peutz-Kootstra, C., Witzke, O., Smits, J. F., and Struijker Boudier, H. A. (2007) Transient AT1 receptor-inhibition in prehypertensive spontaneously hypertensive rats results in maintained cardiac protection until advanced age. J Hypertens 25, 207-215

191. Sanz-Rosa, D., Oubina, M. P., Cediel, E., De las Heras, N., Aragoncillo, P., Balfagon, G., Cachofeiro, V., and Lahera, V. (2005) Eplerenone reduces oxidative stress and enhances eNOS in SHR: vascular functional and structural consequences. Antioxid Redox Signal 7, 1294-1301

192. Melk, A., Schmidt, B. M., Vongwiwatana, A., Rayner, D. C., and Halloran, P. F. (2005) Increased expression of senescence-associated cell cycle inhibitor p16iNK4a in deteriorating renal transplants and diseased native kidney. Am J Transplant 5 , 1375-1382

193. Serrano, A. L., and Andres, V. (2004) Telomeres and cardiovascular disease: does size matter? Circ Res 94, 575-584

194. Rosendorff, C. (1996) The renin-angiotensin system and vascular hypertrophy. J Am Coll Cardiol 28, 803-812

195. Wolf, G., Jablonski, K., Schroeder, R., Reinking, R., Shankland, S. J., and Stahl, R. A. (2003) Angiotensin ll-induced hypertrophy of proximal tubular cells requires p27Kip1. Kidney Int 64, 71-81

196. Nozato, T., Ito, H., Watanabe, M., Ono, Y., Adachi, S., Tanaka, H., Hiroe, M., Sunamori, M., and Marum, F. (2001) Overexpression of cdk Inhibitor p16INK4a by adenovirus vector inhibits cardiac hypertrophy in vitro and in vivo: a novel strategy for the gene therapy of cardiac hypertrophy. J Mol Cell Cardiol 33, 1493-1504

197. Terada, Y., Inoshita, S., Nakashima, O., Tamamori, M., Ito, H., Kuwahara, M., Sasaki, S., and Marumo, F. (1999) Cell cycle inhibitors (p27Kip1 and p21CIP1) cause hypertrophy in LLC-PK1 cells. Kidney Int 56, 494-501

198. Hector Polizio, A., and Pena, C. (2005) Effects of angiotensin II type 1 receptor blockade on the oxidative stress in spontaneously hypertensive rat tissues. Regul Pept 128, 1-5

199. Aviv, A., and Aviv, H. (1997) Reflections on telomeres, growth, aging, and essential hypertension. Hypertension 29, 1067-1072

200. Deppisch, R. M., Beck, W., Goehl, H., and Ritz, E. (2001) Complement components as uremic toxins and their potential role as mediators of microinflammation. Kidney Int. Supp/ 78, S271-S277

201. Brenner, B. M., Lawler, E. V., and Mackenzie, H. S. (1996) The hyperfiltration theory: a paradigm shift in nephrology. Kidney Int. 49, 1774-1777

202. Imig, J. D., Falck, J. R., Gebremedhin, D., Harder, D. R., and Roman, R. J. (1993) Elevated renovascular tone in young spontaneously hypertensive rats. Role of cytochrome P-450. Hypertension 22, 357-364

203. Silveira, L. A., Bacchi, C. E., Pinto, G. A., and De Faria, J. B. (2002) The genetics of hypertension modifies the renal cell replication response induced by experimental diabetes. Diabetes 51, 1529-1534

204. Knox, F. G., Burnett, J. C., Jr., Kohan, D. E., Spielman, W. S., and Strand, J. C. (1980) Escape from the sodium-retaining effects of mineralocorticoids. Kidney Int 17, 
205. Cowley, A. W., Jr. (1997) Role of the renal medulla in volume and arterial pressure regulation. Am J Physio/ 273, R1-15

206. Park, F., Mattson, D. L., Roberts, L. A., and Cowley, A. W., Jr. (1997) Evidence for the presence of smooth muscle alpha-actin within pericytes of the renal medulla. Am J Physiol 273, R1742-1748

207. Mullins, M. M., and Banks, R. O. (1976) Age-related changes in Na+ excretion in saline-loaded spontaneously hypertensive rats. Am.J.Physiol 231, 1364-1370

208. Edwards, A., Delong, M. J., and Pallone, T. L. (2000) Interstitial water and solute recovery by inner medullary vasa recta. Am J Physiol Renal Physiol 278, F257-269

209. Maric, C., Ryan, G. B., and Alcorn, D. (1997) Embryonic and postnatal development of the rat renal interstitium. Anat.Embryol.(Berl) 195, 503-514

210. Hellstrom, M., M, K. n., Lindahl, P., Abramsson, A., and Betsholtz, C. (1999) Role of PDGF-B and PDGFR-beta in recruitment of vascular smooth muscle cells and pericytes during embryonic blood vessel formation in the mouse. Development 126, 3047-3055

211. Badzynska, B., Grzelec-Mojzesowicz, M., Dobrowolski, L., and Sadowski, J. (2002) Differential effect of angiotensin II on blood circulation in the renal medulla and cortex of anaesthetised rats. J Physiol 538, 159-166

212. Bandopadhyay, R., Orte, C., Lawrenson, J. G., Reid, A. R., De Silva, S., and Allt, G. (2001) Contractile proteins in pericytes at the blood-brain and blood-retinal barriers. $J$ Neurocytol 30, 35-44

213. Maric, C., Casley, D., Harris, P., and Alcorn, D. (2001) Angiotensin II binding to renomedullary interstitial cells is regulated by osmolality. J.Am.Soc.Nephrol. 12, 450455

214. Pallone, T. L., Zhang, Z., and Rhinehart, K. (2003) Physiology of the renal medullary microcirculation. Am J Physiol Renal Physiol 284, F253-266

215. Zhang, Z., Rhinehart, K., Kwon, W., Weinman, E., and Pallone, T. L. (2004) ANG II signaling in vasa recta pericytes by PKC and reactive oxygen species. Am J Physiol Heart Circ Physiol 287, H773-781

216. Baumann M, E. H., Hermans JJR, Smits JFM, Struijker Boudier HAJ (2004) Functional and Structural Postglomeruar Aterations in the Kidney of Prehypertensive Spontaneously Hypertensive Rats. Clin Exp Hypertens 26, 663-672

217. Rao, R., Hao, C. M., and Breyer, M. D. (2004) Hypertonic stress activates glycogen synthase kinase 3beta-mediated apoptosis of renal medullary interstitial cells, suppressing an NFkappaB-driven cyclooxygenase-2-dependent survival pathway. $J$ Biol Chem 279, 3949-3955

218. Lu, S., Mattson, D. L., Roman, R. J., Becker, C. G., and Cowley, A. W., Jr. (1993) Assessment of changes in intrarenal blood flow in conscious rats using laser-Doppler flowmetry. Am J Physiol 264, F956-962

219. Gonzalez, A., Lopez, B., Ravassa, S., Querejeta, R., Larman, M., Diez, J., and Fortuno, M. A. (2002) Stimulation of cardiac apoptosis in essential hypertension: potential role of angiotensin II. Hypertension $39,75-80$

220. Dumont, E. A., Reutelingsperger, C. P., Smits, J. F., Daemen, M. J., Doevendans, P. A., Wellens, H. J., and Hofstra, L. (2001) Real-time imaging of apoptotic cellmembrane changes at the single-cell level in the beating murine heart. Nat Med 7, 1352-1355

221. Dumont, E. A., Hofstra, L., van Heerde, W. L., van den Eijnde, S., Doevendans, P. A., DeMuinck, E., Daemen, M. A., Smits, J. F., Frederik, P., Wellens, H. J., Daemen, M. J., and Reutelingsperger, C. P. (2000) Cardiomyocyte death induced by 
myocardial ischemia and reperfusion: measurement with recombinant human annexin- $V$ in a mouse model. Circulation 102, 1564-1568

222. Michea, L., Ferguson, D. R., Peters, E. M., Andrews, P. M., Kirby, M. R., and Burg, M. B. (2000) Cell cycle delay and apoptosis are induced by high salt and urea in renal medullary cells. Am.J.Physiol Renal Physiol 278, F209-F218

223. Pallone, T. L., Turner, M. R., Edwards, A., and Jamison, R. L. (2003) Countercurren1 exchange in the renal medulla. Am J Physiol Regul Integr Comp Physiol 284, R115? 1175

224. Tobian, L., O'Donnell, M., and Ganguli, M. (1978) Relationship of prostaglandins anc sodium in renal papilla in Kyoto hypertensive rats and during high sodium diets. Trans Assoc Am Physicians 91, 204-211

225. De Sarno, P., Li, X., and Jope, R. S. (2002) Regulation of Akt and glycogen synthasi kinase- 3 beta phosphorylation by sodium valproate and lithium. Neuropharmacology 43, 1158-1164

226. Lewington, S., Clarke, R., Qizilbash, N., Peto, R., and Collins, R. (2002) Age-specific relevance of usual blood pressure to vascular mortality: a meta-analysis of individual data for one million adults in 61 prospective studies. Lancet 360, 1903-1913

227. Rizzoni, D., Porteri, E., Boari, G. E., De Ciuceis, C., Sleiman, I., Muiesan, M. L., Castellano, M., Miclini, M., and Agabiti-Rosej, E. (2003) Prognostic significance of small-artery structure in hypertension. Circulation 108, 2230-2235

228. Antonios, T. F., Singer, D. R., Markandu, N. D., Mortimer, P. S., and MacGregor, G. A. (1999) Rarefaction of skin capillaries in borderline essential hypertension suggest an early structural abnormality. Hypertension 34, 655-658

229. Skov, K., Hamet, P., Nyengaard, J. R., and Mulvany, M. J. (2001) Morphology of renal afferent arterioles and glomeruli, heart weight, and blood pressure in primates. Am.J.Hypertens. 14, 331-337

230. Chobanian, A. V., Bakris, G. L., Black, H. R., Cushman, W. C., Green, L. A., Izzo, J. L., Jr., Jones, D. W., Materson, B. J., Oparil, S., Wright, J. T., Jr., and Roccella, E. J. (2003) The Seventh Report of the Joint National Committee on Prevention, Detection, Evaluation, and Treatment of High Blood Pressure: the JNC 7 report. Jama 289, 2560-2572

231. Chobanian, A. V., Bakris, G. L., Black, H. R., Cushman, W. C., Green, L. A., Izzo, J. L., Jr., Jones, D. W., Materson, B. J., Oparil, S., Wright, J. T., Jr., and Roccella, E. J. (2003) Seventh report of the Joint National Committee on Prevention, Detection, Evaluation, and Treatment of High Blood Pressure. Hypertension 42, 1206-1252

232. Zhang, H., Thijs, L., Kuznetsova, T., Fagard, R. H., Li, X., and Staessen, J. A. (2006. Progression to hypertension in the non-hypertensive participants in the Flemish Study on Environment, Genes and Health Outcomes.J Hypertens 24, 1719-1727

233. Node, K., Kitakaze, M., Yoshikawa, H., Kosaka, H., and Hori, M. (1997) Reduced plasma concentrations of nitrogen oxide in individuals with essential hypertension. Hypertension 30, 405-408

234. Tsikas, D. (2004) Mass spectrometry-validated HPLC method for urinary nitrate. Clin Chem 50, 1259-1261

235. Goldblatt, H. (1964) Hypertension of Renal Origin. Historical and Experimental Background. Am J Surg 107, 21-25

236. Arendshorst, W. J., and Beierwaltes, W. H. (1979) Renal tubular reabsorption in spontaneously hypertensive rats. Am J Physiol 237, F38-47

237. Chatziantoniou, C., Daniels, F. H., and Arendshorst, W. J. (1990) Exaggerated renal vascular reactivity to angiotensin and thromboxane in young geneticaliy hypertensive rats. Am J Physiol 259, F372-382 
238. Wang, J. G., Staessen, J. A., Tizzoni, L., Brand, E., Birkenhager, W. H., Fagard, R., Herrmann, S. M., and Bianchi, G. (2001) Renal function in relation to three candidate genes. Am J Kidney Dis 38, 1158-1168

239. Lauer, T., Preik, M., Rassaf, T., Strauer, B. E., Deussen, A., Feelisch, M., and Kelm, M. (2001) Plasma nitrite rather than nitrate reflects regional endothelial nitric oxide synthase activity but lacks intrinsic vasodilator action 10.1073/pnas.221381098. PNAS 98, 12814-12819

240. Lyamina, N. P., Dolotovskaya, P. V., Lyamina, S. V., Malyshev, I. Y., and Manukhina, E. B. (2003) Nitric oxide production and intensity of free radical processes in young men with high normal and hypertensive blood pressure. Med Sci Monit 9, CR304-310

241. Schmidt, R. J., Yokota, S., Tracy, T. S., Sorkin, M. I., and Baylis, C. (1999) Nitric oxide production is low in end-stage renal disease patients on peritoneal dialysis. Am J Physiol 276, F794-797

242. Turi, S., Friedman, A., Bereczki, C., Papp, F., Kovacs, J., Karg, E., and Nemeth, I. (2003) Oxidative stress in juvenile essential hypertension. J Hypertens 21, 145-152

243. Zhang, W., Pibulsonggram, T., and Edwards, A. (2004) Determinants of basal nitric oxide concentration in the renal medullary microcirculation. Am J Physiol Renal Physiol 287, F1189-1203

244. Evans, R. G., and Fitzgerald, S. M. (2005) Nitric oxide and superoxide in the renal medulla: a delicate balancing act. Curr Opin Nephrol Hypertens 14, 9-15

245. Racasan, S., Braam, B., Koomans, H. A., and Joles, J. A. (2005) Programming blood pressure in adult SHR by shifting perinatal balance of $\mathrm{NO}$ and reactive oxygen species toward NO: the inverted Barker phenomenon. Am J Physiol Renal Physiol 288, F626-636

246. Bianchi, G., Niutta, E., Ferrari, P., Salvati, P., Salardi, S., Cusi, D., Colombo, R., Cesana, B., Tripodi, G., Pati, P., and et al. (1989) A possible primary role for the kidney in essential hypertension. Am J Hypertens 2, 2S-6S

247. Bidani, A. K., and Griffin, K. A. (2002) Long-term renal consequences of hypertension for normal and diseased kidneys. Curr Opin Nephrol Hypertens 11, 73-80

248. Paull, J. R., and Widdop, R. E. (2001) Persistent cardiovascular effects of chronic renin-angiotensin system inhibition following withdrawal in adult spontaneously hypertensive rats. $J$ Hypertens $19,1393-1402$

249. Mori, T., and Cowley, A. W., Jr. (2003) Angiotensin II-NAD(P)H oxidase-stimulated superoxide modifies tubulovascular nitric oxide cross-talk in renal outer medulla. Hypertension 42, 588-593

250. Machwe, A., Orren, D. K., and Bohr, V. A. (2000) Accelerated methylation of ribosomal RNA genes during the cellular senescence of Werner syndrome fibroblasts. Faseb J 14, 1715-1724

251. Saxena, A. K., Saxena, P., Wu, X., Obrenovich, M., Weiss, M. F., and Monnier, V. M. (1999) Protein aging by carboxymethylation of lysines generates sites for divalent metal and redox active copper binding: relevance to diseases of glycoxidative stress. Blochem Biophys Res Commun 260, 332-338

252. Garcia-Saura, M. F., Galisteo, M., Villar, I. C., Bermejo, A., Zarzuelo, A., Vargas, F., and Duarte, J. (2005) Effects of chronic quercetin treatment in experimental renovascular hypertension. Mol Cell Biochem 270, 147-155

253. Cawthon, R. M. (2002) Telomere measurement by quantitative PCR. Nucleic Acids Res 30 , e47

254. Abshagen, U., Rennekamp, H., and Luszpinski, G. (1976) Pharmacokinetics of spironolactone in man. Naunyn Schmiedebergs Arch Pharmacol 296, 37-45 
255. Obata, J., Nakamura, T., Takano, H., Naito, A., Kimura, H., Yoshida, Y., Shimizu, F., Guo, D. F., and Inagami, T. (2000) Increased gene expression of components of the renin-angiotensin system in glomeruli of genetically hypertensive rats. J.Hypertens. $18,1247-1255$

256. Houben, A. J., Burgwinkel, J. P., and de Leeuw, P. W. (2006) A novel approach to the study of human microcirculation: Reactivity to locally applied angiotensin II in the conjunctival microvascular bed. J Hypertens 24, 2225-2230

257. Cowley, A. W., Jr., and Roman, R. J. (1996) The role of the kidney in hypertension. Jama 275, 1581-1589

258. Cowley, A. W., Jr., Mori, T., Mattson, D., and Zou, A. P. (2003) Role of renal NO production in the regulation of medullary blood flow. Am J Physiol Regul Integr Comp Physiol 284, R1355-1369

259. Dickhout, J. G., Mori, T., and Cowley, A. W., Jr. (2002) Tubulovascular nitric oxide crosstalk: buffering of angiotensin II-induced medullary vasoconstriction. Circ Res 91 , 487-493

260. Kietselaer, B. L., Reutelingsperger, C. P., Heidendal, G. A., Daemen, M. J., Mess, W. H., Hofstra, L., and Narula, J. (2004) Noninvasive detection of plaque instability with use of radiolabeled annexin A5 in patients with carotid-artery atherosclerosis. $N$ Engl J Med 350, 1472-1473

261. Boersma, H. H., Kietselaer, B. L., Stolk, L. M., Bennaghmouch, A., Hofstra, L., Narula, J., Heidendal, G. A., and Reutelingsperger, C. P. (2005) Past, present, and future of annexin A5: from protein discovery to clinical applications. J Nucl Med 46, 2035-2050

262. Zhang, Z., and Pallone, T. L. (2004) Response of descending vasa recta to luminal pressure. Am J Physiol Renal Physiol 287, F535-542

263. Navar, L. G., Harrison-Bernard, L. M., Imig, J. D., Wang, C. T., Cervenka, L., and Mitchell, K. D. (1999) Intrarenal angiotensin II generation and renal effects of AT1 receptor blockade. J.Am. Soc.Nephrol. 10 Suppl 12, S266-S272

264. Esther, C. R., Jr., Howard, T. E., Marino, E. M., Goddard, J. M., Capecchi, M. R., and Bernstein, K. E. (1996) Mice lacking angiotensin-converting enzyme have low blood pressure, renal pathology, and reduced male fertility. Lab Invest 74, 953-965

265. Amlal, H., LeGoff, C., Vernimmen, C., Soleimani, M., Paillard, M., and Bichara, M. (1998) ANG II controls $\mathrm{Na}(+)-\mathrm{K}+(\mathrm{NH} 4+)-2 \mathrm{Cl}$ - cotransport via $20-\mathrm{HETE}$ and $\mathrm{PKC}$ in medullary thick ascending limb. Am.J.Physiol 274, C1047-C1056

266. Robey, R. B., Ruiz, O. S., Espiritu, D. J., Ibanez, V. C., Kear, F. T., Noboa, O. A., Bernardo, A. A., and Arruda, J. A. (2002) Angiotensin II stimulation of renal epithelial cell $\mathrm{Na} / \mathrm{HCO} 3$ cotransport activity: a central role for Src family kinase/classic MAPK pathway coupling. J Membr Biol 187, 135-145

267. Good, D. W., George, T., and Watts, B. A., 3rd (2002) Aldosterone inhibits HCO absorption via a nongenomic pathway in medullary thick ascending limb. Am J Physiol Renal Physiol 283, F699-706

268. Peti-Peterdi, J., Warnock, D. G., and Bell, P. D. (2002) Angiotensin II directly stimulates ENaC activity in the cortical collecting duct via AT(1) receptors. J Am SoC Nephrol 13, 1131-1135

269. Rao, R., Zhang, M. Z., Zhao, M., Cai, H., Harris, R. C., Breyer, M. D., and Hao, C. M. (2005) Lithium treatment inhibits renal GSK-3 activity and promotes cyclooxygenase 2-dependent polyuria. Am J Physiol Renal Physio/ 288, F642-649

270. Racasan, S., Joles, J. A., Boer, P., Koomans, H. A., and Braam, B. (2003) NO dependency of RBF and autoregulation in the spontaneously hypertensive rat. $A m \mathrm{~J}$ Physiol Renal Physiol 285, F105-112 


\section{ACKNOWLEDGEMENT}

Now I am sitting here and finalize my thesis. I left this part over till the end as the acknowledgement represents a great challenge for me. I am somewhat afraid that I forget someone. So I have to decide whether I write here a detailed acknowledgement or keep it more general and thus shorter.

The first time I came to Maastricht I never had thought before about a Ph.D. thesis. My aim was to fulfill some time abroad such as a "typical German medical doctor" and than to go back to my German University clinic. I guess only Harry thought at that time that I would go for a thesis. Harry is the central person for my being here today as he gave me the chance to find my way and secondly give myself time to develop. And that makes already the difference to many of my German colleagues who became fellows somewhere else. While others followed someone, he taught me to find my own way. This is of course no fast track, but it allowed me to build my own character. In this context, I would like to thank the committee which had to assess my book as result of my development.

And this little difference changed a lot in my life. Now I am neither a "typical medical doctor" nor a "typical German" anymore. I rather became a mixture between scientist and medical doctor and my German characters were added up by Dutch soft skills (I still unsure whether my new German colleagues love or hate me for this...).

There the point comes where I have to become more general. I don't want to point out someone and to forget another one. Those who have to be pointed out must apologize me, but they know about their importance anyhow. Thus, I would like to thank heartily all my colleagues in the department of pharmacology and toxicology: You taught me to organize myself and most importantly brought Dutch "gezelligheid" to me. I proudly wear the University Maastricht sweatshirt and the yellow plastic tulip stands on my table in the lab, whereas The Netherlands in chocolate form do not exist anymore...

Finally thanks to my wife Shi-Hui, my daughter Yili and my parents: Without their "okay" to move to Maastricht and to live in Aachen nothing would have happened. Thanks for the "allowance". Actually, our stay in Maastricht was the longest in my last 15 years. Thus, 1 guess it was not the worst decision for us. 


\section{LIST OF PUBLICATIONS}

\section{Full Papers}

Kanzler M, Baumann M, Schirmacher P, Dries V, Bayer E, Gerken G, Dienes HP, Lohse AW. Prediction of progressive liver fibrosis in hepatitis $C$ infection by serum and tissue levels of transforming growth factor-bela. J Viral Hepat. 2001; 8(6): 430-7.

Wang M, Bai J, Baumann M, Heemann U. New model for simultaneous heart and kidney transplantation in mice. Microsurgery. 2003; 23(2): 164-8.

Struijker Boudier HA, Cohuet GM, Baumann M, Safar ME. The heart, macrocirculation and microcirculation in hypertension: a unifying hypothesis. J Hypertens Suppl. 2003; 21Suppl 3: S19-23.

Baumann M, Witzke O, Haug U, Deppisch R, Lutz J, Philipp T, Heemann U. Prolonged catheter survival in intermittent haemodialysis by reducing blood-material contact. ASAIO J. 2003; 49(6): 708-12.

Baumann $M$, Niebel $W$, Kribben A, Philipp T, Heemann U. Primary failure of arteriovenous fistulae in auto-immune disease, Kidney Blood Press Res. 2003; 26(5-6): 362-7.

Baumann M, van Essen H, Hermans JJR, Smits JFM, Struijker- Boudier HAJ. Functional and structural postglomerular alterations in the kidney of prehypertensive spontaneously hypertensive rats. Clin Exp Hypertens. 2004; 26(7-8): 663-72.

Baumann M, Witzke O, I Patschan D, Patschan S, Mitchell A, Philipp T, Kribben A. Which patients benefit from hemodialysis therapy in hepatorenal syndrome? J Gastroenterol Hepatol. 2004; 19(12): 136973.

Baumann M, Witzke O. Philipp T, Kribben A. Serum C3 complement concentrations correlate with liver function in patients with liver cirrhosis. Hepatogastroenterology. 2004; 51(59): 1451-3.

Schellings MW, Baumann $M^{*}$, van Leeuwen RE, Duisters RF, Janssen SH, Schroen B, Peutz-Kootstra CJ, Heymans S, Pinto YM: Imatinib attenuates end-organ damage in hypertensive homozygous TGR(mRen2)27 rats. Hypertension 2006; 47: 467-74. shared $1^{\text {st }}$ autorship

Baumann M, van den Born. Treating prehypertension. N Engl J Medicine 2006: 344(4): 316-318.

Baumann M. Prehypertensive treatment. Vasculaire Geneeskunde 2006: 4: 17-18. 
Baumann M, Janssen BJ, Hermans JR, Peutz-Kootstra C, Witzke O, Smits JF, Struijker Boudier HAJ. Transient AT1 receptor-inhibition in prehypertensive spontaneously hypertensive rats results in maintained cardiac protection until advanced age. J Hypertens 2007: 25(1): 207-215.

Bogaard B, Baumann M, van Weert HCPM, Struijker Boudier HAJ, Montfrans GA, van den Born BJH. De TIResiAS studie. NTVG 2007: 151(32): 1800-1801.

Baumann M, Megens R, Bartholome R, Dolff S, van Zandvoort M, Smits JFM, Struijker Boudier HAJ, de Mey JGR. Prehypertensive treatment ameliorates endothelial dysfunction in aged SHR. Hypertension Res 2007: 30: 853-861.

Baumann M, Hermans R, Janssen B, Peutz-Kootstra C, Smits J, Struijker Boudier HAJ. Transient prehypertensive treatment in SHR: A comparison of spironolactone and losartan regarding long-term blood pressure and target organ damage. Journal of Hypertension in press

\section{Published Abstracts}

Baumann M, Witzke O, Haug U, Deppisch R, Lutz J, Philipp T, Heemann U. Prolonged catheter survival in intermittent haemodialysis by reducing blood-material contact. Kidney Blood Press Res 2001; 24: 308 171.

Baumann M, Niebel W, Kribben A, Philipp T, Heemann U. Primary failure of arteriovenous fistulae in auto-immune disease. Kidney Blood Press Res 2001; 24: 308(72).

Baumann M, Witzke O, Eder D, Mitchell A, Philipp T, Kribben A. Effectiveness of dialysis in Hepatorenal syndrome (HRS). Kidney Blood Press Res 2002; 25: 308(175).

Baumann M, van Essen H, Hermans JJR, Philipp T, Smits JFM, Struijker Boudier HAJ. Increased number of alpha-actin positive vasa recta in prehypertensive SHR. Hypertension 2002; 40(4): 591.

Baumann $M$, van Essen $H$, Hermans JJR, Philipp T, Smits JFM, H Struijker Boudier HAJ.In vivo plastination of $M$. cremaster and kidney - a feasible method for visualization of the arteriolar tree. Hypertension 2002; 40(4): 591.

Baumann M, Hermans JJR, Smits JFM, Struijker Boudier HAJ. Early transient aldosterone antagonism prevents the development of hypertension in spontaneously hypertensive rats (SHR) Hypertension 2003; 42: 312 .

Baumann M, Hermans JJR, Smits JFM, Struijker Boudier HAJ. Early transient TGF- $\beta_{1}$-inhibition alters intrarenal growth and increases blood pressure in spontaneously hypertensive rats (SHR) Hypertension 2003; $42: 334$. 
Baumann M, Hermans JJR, Smits JFM, Strujiker Boudier HAJ.. Prehypertensive RAS-inhibition: Renoprotection by inhibition of inflammatory phenotype? Hypertension 2004; 44: 192.

Baumann M, Hermans JJR, Debets J, Wesselman JPM, Fazzi GE, De Mey JGR, Smits JFM, Struijker Boudier HAJ. Role of aldosterone on the development of hypertension in SHR. Hypertension 2004; 44: 193.

Baumann M, Schellings MWM, Heeneman S, Heeringa P, Daemen MJ, Pinto YM, Peutz-Kootstra CJ. Renal damage in hypertensive homozygous Ren 2 rats is attenuated by imatinib treatment. Kidney Inter $2005 ; 67: 1562$.

Baumann M, Jos FM Smits, Harry AJ Struijker Boudier. Long-term echocardiographic follow-up of brief and early losartan treated SHR reveals sustained cardiac protection. Hypertension 2005; $46: 896$.

Baumann M, Schalkwijk C, Stehouwer C, Schleijen C, Harry AJ Struijker Boudier. CML is increased in the kidney from spontaneously hypertensive rats and is reduced by losartan and spironolactone, but not by hydralazine. Hypertension 2006; 49: 267.

Baumann M, Jos FM Smits, Harry AJ Struijker Boudier. Advanced renoprotection in SHR by transient prehypertensive treatment: an effect of decelerated aging? Hypertension 2006; 49: 269.

Baumann M, Jos FM Smits, Harry AJ Struijker Boudier, de Mey J. Prehypertensive treatment ameliorates endothelial dysfunction in aged SHR. Hypertension 2006; 49: 270.

Baumann M, Kouznetsova T, Bartholome R, Smits J, Struijker Boudier H, Staessen J. Potential individual prediction of the progression of the progression from prehypertension to new-onset hypertension. $J$ Hypertens 2007; 25(suppl 2): 97.

Baumann M, Dan L, Kribben A, Heemann U, Witzke O. Thyroid gland associated stiffness deteriorates circadian blood pressure rhythm. J Hypertens 2007; 25(suppl 2): 204.

Baumann M, Dan L, Kribben A, Heemann U, Witzke O. Aortic stiffness and kidney function. J Hypertens 2007; 25(suppl 2): 204.

\section{Awards}

European Council of Cardiovascular Research (ECCR): Young Investigator Award 2003

European Council of Cardiovascular Research (ECCR): Young Investigator Award 2004 
Meeting of the Dutch-Danish Pharmacology Societies: Poster Prize 2006

International Society of Hypertension (ISH): Young Investigator Award 2006 


\section{CURRICULUM VITAE}

Marcus Baumann was born on 27th of October 1973 in Wuppertal, Germany. After he finished his Abitur at the Wilhelm Dörpfeld Gymnasium in Wuppertal he started in 1993 to study Medicine at the Johannes Gutenberg-University of Mainz, Germany. During this time he studied under his promotores Meyer zum Büschenfelde and Lohse the prediction of progressive liver fibrosis in hepatitis $C$ infection by serum and tissue levels of transforming growth factor- $\beta$. He finished this work with the German medical doctor degree successfully with magna cum laude. After his last medical year at the Queen Elisabeth Hospital, Hongkong, he began in 2000 his internship in medicine at the University Hospital Essen, Germany.

Starting from 2002 until end 2006 he worked as medical researcher at the Department of Pharmacology and Toxicology under Harry AJ Struijker Boudier and Jos FM Smits investigating the in this book described work. The studies were supported by an EU Marie Curie grant, followed by a grant support by the Dutch Kidney Foundation. In relation to the here described work a cooperation with several Dutch groups was started and a grant was successfully applied by ZonMW to investigate the effectiveness of prehypertensive treatment in humans. The study is called TIRESIAS and was started 2007. Since 2007 he works at the Technical University of Munich at the Klinikum rechts der Isar in the Department of Nephrology continuing his research. 